


\section{e $\because 0$ che}

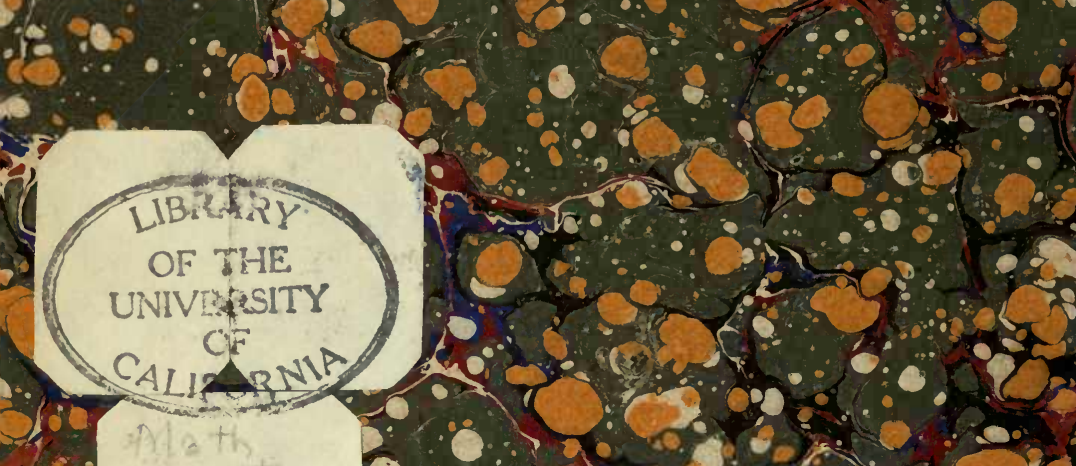
50 bota

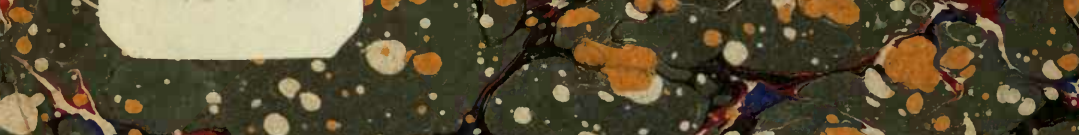

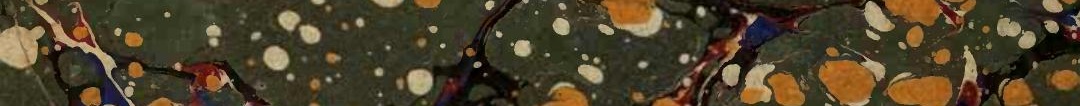
ob. 000.4500

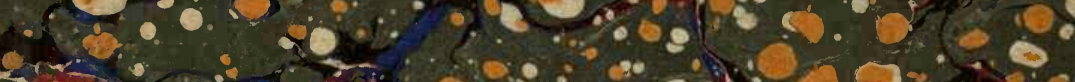

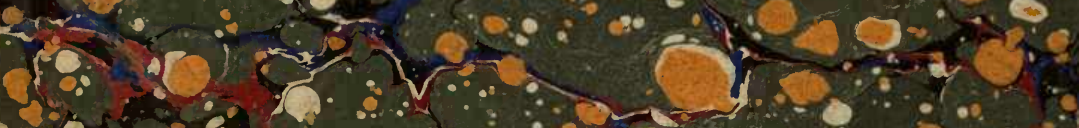

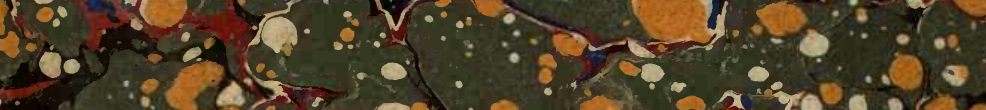

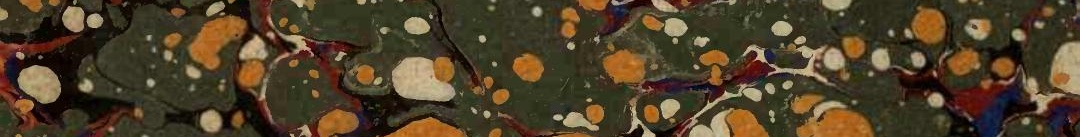
s. a jolos:

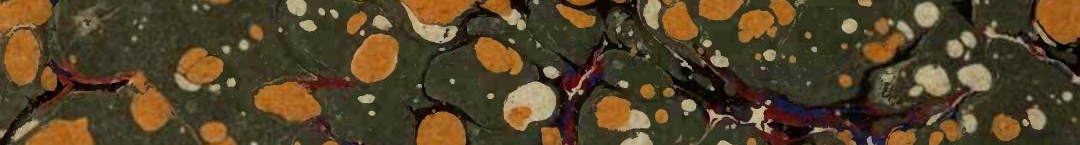

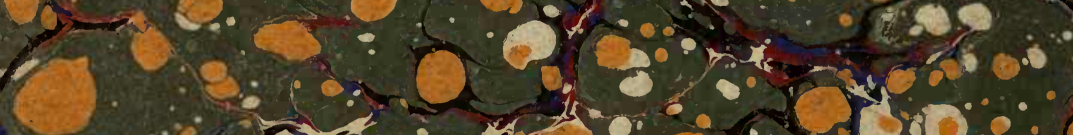
- 0 Pी

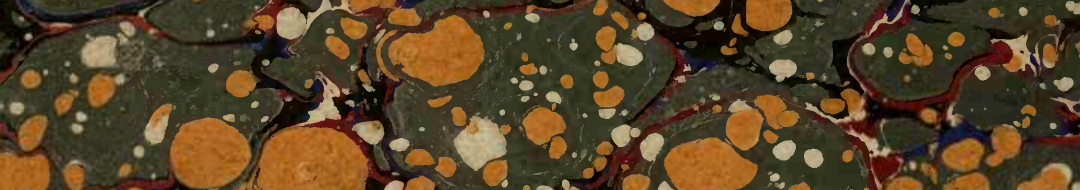

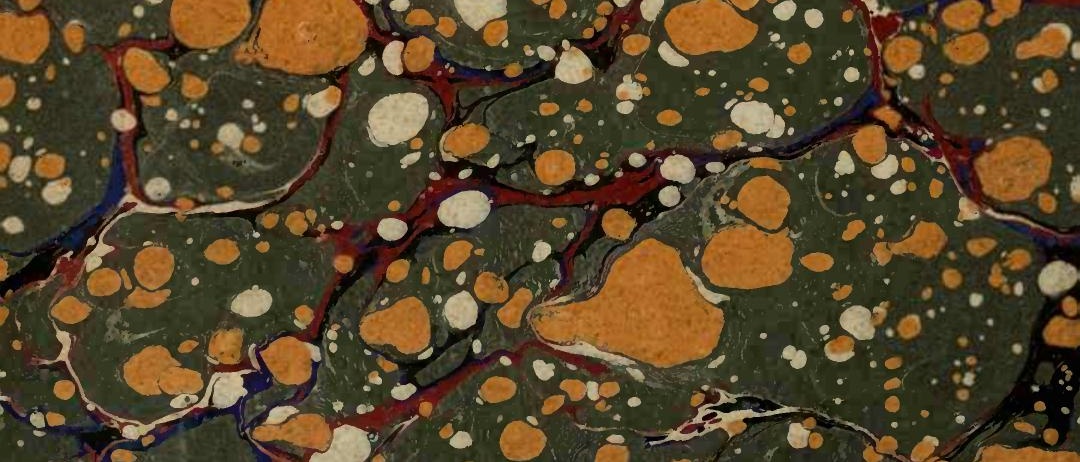


LIBRAR Y OF THE UNIVERSIT CAuTORnL MATH.STAT, 
MW. Yasku:

1896 . 


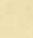

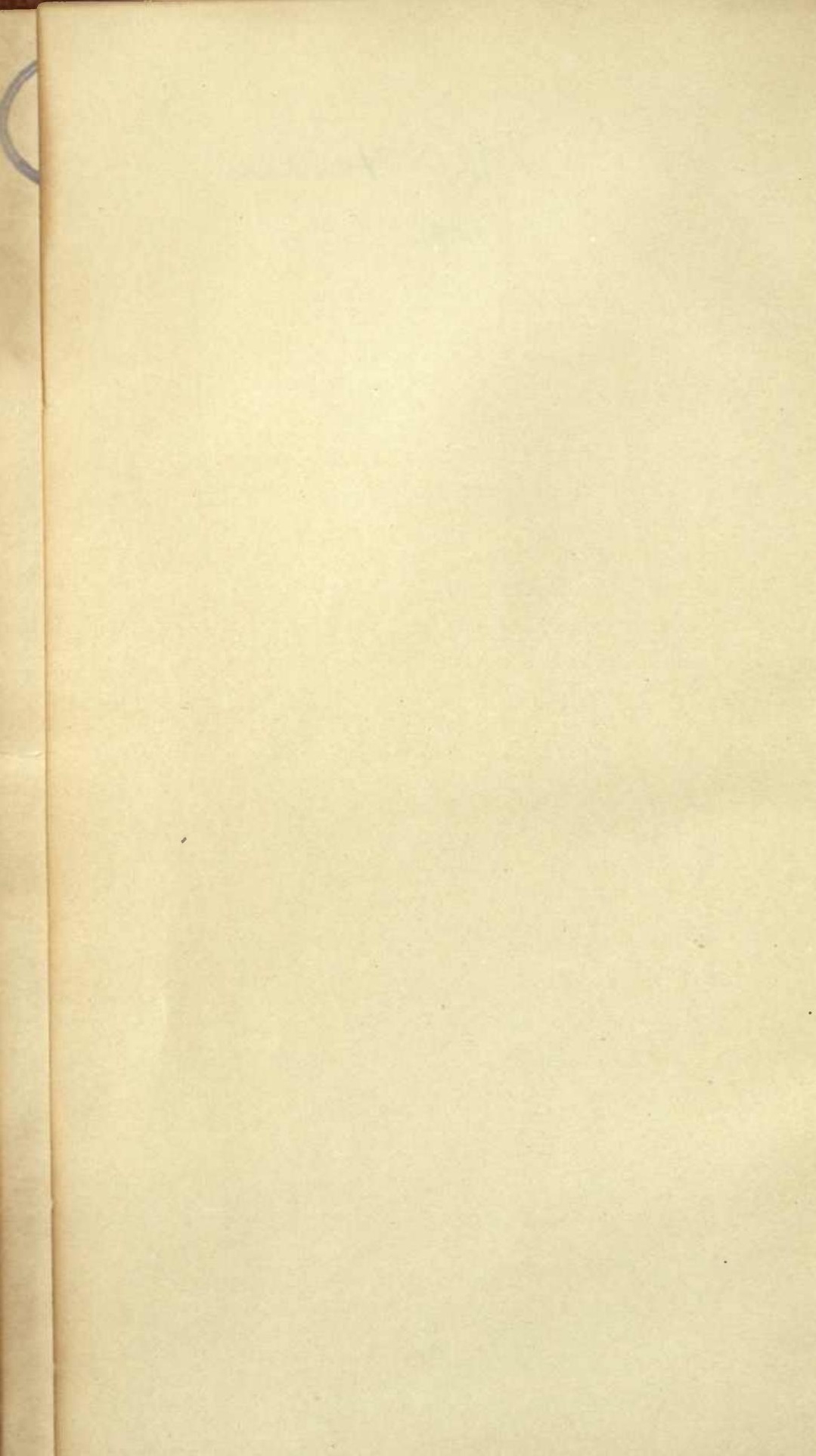




GESCHICHTE DER MATHEMATIK

ALTERTUM UND MITTELALTER 
VERLAG VON ANDR. FRED. HÖST \& SÖN IN KOPENHAGEN

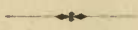

\section{DIE LEHRE}

YON DEN

\section{KEGELSCHNITTEN IM ALTERTUM}

voN

Prof. Dr. H. G. ZEUTHEN

Preis 15 Mark 


\title{
ALTERTUM UND MITTELALTER
}

\author{
VORLESUNGEN
}

VON

\section{H. G. ZEUTHEN}

PROFESSOR AN DER UNIVERSITÄT KOPENHAGEN

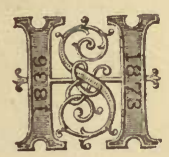

\section{KOPENHAGEN}

VERLAG VON ANDR. FRED. HÖST \& SÖN 


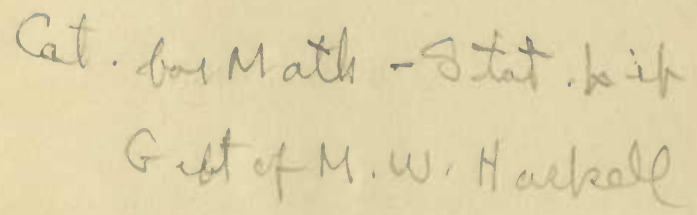

ALLE RECHTE VORBEHALTEN

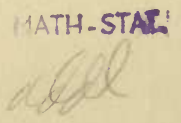

Kopenhagen. - Grobes Buchdruckerei 


\section{QA21 \\ 248 \\ MATH.: \\ STAT. \\ LIBRARY}

\section{Vorrede zur dänischen Ausgabe.}

In der hier vorliegenden Geschichte der Mathematik habe ich versucht namentlich dasjenige hervorzuheben, was für Studenten und Lehrer der Mathematik zu wissen wichtig ist. Für diese kommt es nicht so sehr darauf an viele historische Einzelheiten zu kennen, zu wissen, wer zuerst diese oder jene Wahrheit entdeckt oder dieses oder jenes Verfahren erfunden hat, sondern vielmehr darauf die Formen zu kennen, unter denen die verschiedenen Wahrheiten und Verfahrungsarten entstanden sind, sowie die Anwendungen, die man von ihnen gemacht hat. Die richtige Erkenntnis ihres Ursprunges wird zugleich die Bedingung sein für das Verständnis der Entwickelung, die sie fernerhin durchgemacht haben, bis sie nach und nach der Mathematik ihre jetzige Gestalt gegeben haben.

Indem ich namentlich hierauf Gewicht lege, befinde ich mich in guter Übereinstimmung mit dem Programm für das Schulamtsexamen in Mathematik. Wenn darin nämlich verlangt wird "ein kurzer Abriss der Geschichte der Mathematik, im Anschluss an den der Kandidat sich direkt mit Euklids Elementen und Descartes' Geometrie bekannt gemacht haben muss», so weist diese Forderung 
darauf hin, dass ein solches Verständnis der Geschichte der Mathematik verlangt wird, wie es nur durch einige Bekanntschaft mit der Mathematik der entschwundenen Zeiten erreicht werden kann. In dem vorliegenden Bande, der nur Altertum und Mittelalter behandelt, habe ich es von den beiden genannten Schriftstellern nur mit Euklid $\mathrm{zu}$ thun. Indem ich unter ausdrücklichem Hinweise auf lie einzelnen Sätze die Stellen anführe und erkläre, aus denen namentlich etwas zu lernen ist, suche ich eine fruchtbare Bekanntschaft mit ihm zu vermitteln. Ferner suche ich im Anschluss hieran verständlich $\mathrm{zu}$ machen, was ich von den übrigen Schriftstellern mitzuteilen habe, von denen ich nicht erwarten kann, dass die Leser sie in die Hände bekommen. Unter anderem habe ich Euklids Elemente benutzt zur Erklärung der logischen Formen, die von den griechischen Mathematikern so strenge beobachtet werden. Ich habe mich dabei nicht nur an die Bedeutung gehalten, die diese Formen für die Griechen hatten, sondern zugleich - in Zusätzen mit kleinerem Druck - geprüft, welche Bedeutung ihnen an und für sich zukommt. Ich hoffe dadurch namentlich den Lehrern Veranlassung gegeben $\mathrm{zu}$ haben unter ihnen zu wählen und auszuscheiden, denn zu beiden Dingen ist Veranlas. sung vorhanden.

Wenn ich nun auch mit dem hier ausgesprochenen Zweck vor Augen keineswegs beabsichtige dem historischen Rahmen eine sehr grosse Ausdehnung zu geben, so muss dieser doch so gut und zuverlässig sein wie möglich. Das wird leicht erreicht durch Benutzung von Cantors Vorlesungen über die Geschichte der Mathematik, eines Wer kes, das alle faktischen Aufklärungen, die sich herbeischaffen lassen, mit ungewöhnlicher Vollständigkeit und Zuverlässigkeit giebt. Von diesen Eigenschaften habe ich auch während der Untersuchungen Nutzen ziehen können, 
die nach dem Plane meines Buches zunächst meine eigene Arbeit ausmachen mussten. Allerdings musste das Material für diese vorzugsweise dem Studium der bedeutendsten mathematischen Schriftsteller aus den Zeiten, die ich behandle, entnommen werden; aber während dieses Studiums gewährten mir Cantors Auszüge gute Anleitung um das zu finden, was ich brauchte, selbst dann, wenn ich es nachher anders aufgefasst und benutzt habe als er. $\mathrm{Zu}$ gleich wusste ich, dass ich mich auf seine Mitteilungen über den Inhalt der weniger bedeutenden Werke sicher verlassen konnte, die mir nicht zugänglich waren oder mit denen mich direkt bekannt zu machen es mir an Gelegenheit fehlte. Ich habe jedoch nicht allein in rein thatsächlichen Fragen auf Cantor gebaut. An seine Beurteilungen habe ich mich zum grossen Teil gehalten mit Bezug auf die Zeiten, wo die Mathematik entweder nur Rückschritte machte oder doch keine von den wirklichen Fortschritten, deren Verfolgung meine Aufgabe war. Was ich beispielsweise über das Rechnen auf dem Abacus im Mittelalter und seinen Ursprung mitteile, sind die Resultate von Cantors sorgfältigen und scharfsinnigen Untersuchungen.

Im übrlgen ist es selbstverständlich, dass, während ich mein Studium auf die überlieferten Werke der grossen Mathematiker, auf ihren Zusammenhang unter einander und mit den mathematischen Arbeiten und Resultaten, die nur aus Berichten bekannt sind, richtete, ich die Hülfe benutzt habe, die in der heutigen reichen Litteratur über die Geschichte der Mathematik geboten war. Der didaktische Charakter des vorliegenden Buches hat mich jedoch davon abgehalten anzuführen, was ich diesem oder jenem verdanke. Um das zu thun hätte ich näm. lich nicht nur den Gedanken oder die Auffassung darstellen müssen, die ich dem betreffenden unmittelbar 
verdankte, sondern auch über die Modifikationen berichten müssen, die solche Gedanken unter meiner weiteren Bearbeitung erfahren hatten und über die Gründe hierfür. Dazu war kein Raum, vielmehr musste ich mich damit begnügen kurz die Gründe für die Auffassungen anzuführen, bei denen ich stehen geblieben war. An einem anderen Orte jedoch habe ich mich einer solchen eingehenden Diskussion, bei der ich auch Gelegenheit hatte auszusprechen, was ich jedem einzelnen Schriftsteller verdankte, nicht entzogen, nämlich in meiner Arbeit über die Lehre von den Kegelschnitten im Altertum (Kgl. Danske Videnskabernes Selskabs Skrifter, 6. Række, 3. Bind; deutsche Ausgabe von R. v. Fischer-Benzon, Kopenhagen, Andr. Fred. Høst \& Søn, 1886). Allerdings wird hierin nur die höhere Geometrie des Altertums behandelt, aber diese hing selbstverständlich so genau mit der elementaren Mathematik zusammen, dass ich auch wesentliche Seiten meiner Auffassung von dieser begründen musste, und diese Auffassung steht wieder in genauem Zusammenhange mit fast allem, was ferner in diesem Bande erwähnt wird.

Deshalb begnüge ich mich im wesentlichen damit an dieser Stelle die Männer anzuführen, deren Arbeiten über die Geschichte der Mathematik Einfluss verschiedener Art auf meine eigenen Studien und dadurch auf die vorliegende Arbeit gehabt haben: Chasles, Brettschneider, Hankel, Cantor, P. Tannery, Heiberg, Allmann ${ }^{1}$, und als Herausgeber, Übersetzer und Kommentatoren wieder Heiberg, Hultsch, Wertheim, Colebrooke, Woepcke, Boncompagni. Von Einzelheiten muss ich jedoch hinzufügen, dass ich ausser vielem anderen, was

1 Soria's umfassendes Werk, Le Scienze esatte nell'antica Grecia 1-2, lag damals noch nicht vor. 
zumteil in der Lehre von den Kegelschnitten im Altertum erwähnt ist, die Erklärung (S. 33) des Berichtes über die Geometrie des Thales P. Tannery (Géométrie grecque S. 89 ff.) verdanke, ebenso wie die Erklärung (S. 64, 65) von Pythagoras' Satz, dass «die Dinge Zahlen sind» (Géom. gr. S. 124). Ferner muss ich noch anführen, dass desselben Verfassers gründliches und geistreiches Werk, Recherches sur l'astronomie ancienne, Paris 1893, allerdings erst in meine Hände gelangte, nachdem der Druck meines Buches begonnen war, dass es mich aber dennoch veranlasste die damals noch ungedruckten Abschnitte über die berechnende und sphärische Geometrie der Griechen umzuarbeiten. In einem Buche wie dem vorliegenden konnte ich jedoch nur mit einiger Vorsicht das benutzen, was er selbst ausdrücklich als Hypothesen bezeichnet, wie natürlich auch die Erklärung scheinen mag, die diese Hypothesen von Verhältnissen geben, über deren genauen Zusammenhang in der überlieferten Litteratur keine hinreichenden Angaben vorliegen.

Kopenhagen, im September 1893.

\section{Vorrede zur deutschen Ausgabe.}

Wie aus der obenstehenden Vorrede zur dänischen Ausgabe hervorgeht, ist das vorliegende Buch ursprünglich dazu bestimmt, von Studenten an unserer dänischen Universität benutzt $\mathrm{zu}$ werden. In Übereinstimmung mit dem Programm für das Schulamtsexamen an dieser treten recht bedeutende Abschnitte nahezu als Kommentare zu Euklids Elementen auf, und es ist vorausgesetzt, dass der 
Leser selbst imstande ist die angeführten Stellen in diesen nachzusehen. Das wird jedoch kaum ein Hindernis dafür sein, mein Buch auch ausserhalb Dänemarks zu benutzen; denn um das rechte Verständnis für die Entwickelung der Mathematik überhaupt zu gewinnen, muss man aus eigener Anschauung wenigstens das Werk kennen, das während dieser ganzen Entwickelung die Hauptrolle gespielt hat.

Grösseres Bedenken sollte es vielleicht in mir hervorrufen, dass ich versuche einem historischen Buche, das in historischer Beziehung auf den Arbeiten von Zeitgenossen aufgebaut ist, ausserhalb der Kreise, für die es zuerst bestimmt war, Verbreitung zu verschaffen. Indessen ist dies Buch zugleich eine Frucht selbständiger und persönlicher Arbeit, aber von mehr mathematischer Art, nämlich eines eingehenden Studiums der grossen Schriftsteller aus den Zeiten, die erwähnt werden. Dieses Studium, bei dem ich mich nicht mit solchen Thatsachen begnügen wollte, wie dass dieser oder jener Schriftsteller diesen oder jenen Satz kannte, auch nicht damit, dass er ihn auf diese oder jene Weise beweist, sondern auch versucht habe zu verstehen, weshalb Satz und Beweis unter damaligen Verhältnissen gerade in dieser oder jener Gestalt auftreten mussten, hat mir selbst so viel Zeit und Nachdenken gekostet, dass ich es für nützlich halten darf, die gewonnene Ausbeute für solche darzustellen, die nicht in der Lage sind die erforderliche Zeit und Arbeit auf ein solches Studium zu verwenden.

Da die Aufgabe, die ich mir gestellt habe, in manchem mit derjenigen zusammenfällt, die $\mathrm{Hankel}$ in seinem Buche Zur Geschichte der Mathematik im Altertum und Mittelalter, Leipzig 1874, behandelt hat, so beeile ich mich zu bemerken, dass gerade dieses geistreiche Werk in mir den Geschmack an solchen Studien hervor- 
gerufen hat. Dass meine Arbeit dennoch nicht überflüssig sein werde, hoffe ich teils deshalb, weil Hankels früher Tod die Behandlung mehrerer der wichtigsten Abschnitte verhindert hat, teils deshalb, weil ich, der ich auf neuere historische Untersuchungen habe fussen können, in mancher Beziehung zu anderen Ergebnissen gelangt bin. Unter den Schriftstellern der Gegenwart ist Paul Tannery derjenige, dessen Auffassung ich am häufigsten geglaubt habe mir aneignen $\mathrm{zu}$ müssen. Die wichtigste von den Änderungen, die in der vorliegenden Ausgabe vorgenommen worden sind, besteht darin, dass ich mich jetzt an die neuesten Ermittelungen über Heros Lebenszeit habe halten können, Ermittelungen, die so vortrefflich zu dem Eindruck stimmen, den die überlieferten Werke dieses Schriftstellers in uns hervorrufen. Ausserdem habe ich jetzt etwas mehr Rücksicht auf Tannerys Recherches sur l'astronomie ancienne nehmen können als in der dänischen Ausgabe.

Schliesslich möchte ich meine Freude darüber zum Ausdruck bringen, auch diese Übersetzung von Professor v. Fischer-Benzons kundiger und sorgfältiger Hand ausgeführt $\mathrm{zu}$ sehen.

Kopenhagen, im Juli 1895.

H. G. Zeuthen. 



\section{EINLEITUNG.}

\section{Vorgeschichte der Mathematik.}

Bei einer historischen Vorlesung erhebt sich immer die Frage, wo man zu beginnen habe. Man kann dort anfangen, von wo an zuverlässige positive Angaben vorliegen, die unmittelbar ein zuverlässiges positives Wissen gewähren, und es ist dann Aufgabe des Historikers hierin den richtigen Zusammenhang herzustellen; oder man kann mit der Vorgeschichte anfangen, und diese ist dann abzuleiten durch Kombinieren einer Menge äusserst verschiedener Nachrichten und Thatsachen, die, einzeln genommen, nicht als historische Quellen betrachtet werden können. Unter diesen kommen die Traditionen und Sagen über Sitten und Begebenheiten in noch älteren Zeiten, die sich in den ältesten wirklichen Überlieferungen vorfinden, wirklicher Geschichte am nächsten. Die Erklärung dieser Sagen muss sich stützen auf solche aufgefundene Gegenstände, die in jenen alten Zeiten hervorgebracht und gebraucht worden sind. Die Bedeutung solcher Funde ist ins Licht zu setzen durch ihr gegenseitiges Verhältniss in Bezug auf Zeit und Ort. Ungekehrt tragen sie wieder dazu bei die Verbindungen klar zu legen, die zwischen den Fundstellen bestanden haben, und die zeitliche Aufeinanderfolge der Geschlechter zu bestimmen, die sie be- 
nutzt haben. Was sich in diesen verschiedenen Beziehungen über aufbewahrte äussere Dinge sagen lässt, das gilt auch für diejenigen Worte der Sprache, deren Alter aus ihrem Vorkommen in verschiedenen bekannten lebenden und toten Sprachen hervorgeht. Diese zeugen von alter Bekanntschaft mit entsprechenden Begriffen.

Bei dẹ Behandlung des auf solche Weise gewonnenen Stoffes muss man wieder zu anderen Hülfsquellen seine $\mathrm{Zu}$ flucht nehmen. Um sich eine Meinung darüber zu bilden, wie ein Gegenstand hervorgebracht und gebraucht, wie ein $\mathrm{Be}$ griff entstanden und mit anderen in Verbindung gesetzt worden ist, muss man zuerst wissen, wie derartiges überhaupt geschehen kann und wie es mit den Hülfsmitteln und der Auffassung der Dinge, die man als damals üblich annehmen darf, hat geschehen können. Oft ist es schwierig hierüber zu urteilen, namentlich für einen Kulturmenschen der Gegenwart, der gewohnt ist sich einer Menge jetzt existierender, körperlicher und geistiger Hülfsmittel oder angeeigneter Fertigkeiten zu bedienen, ohne selbst recht zu wissen, welchen Nutzen sie ihm gewähren, oder welche von ihnen leicht und welche schwierig durch andere ersetzt werden könnten. Um darüber etwas $\mathrm{zu}$ erfahren, sind wir darauf angewiesen teils unsere eigenen Kinder zu beobachten, teils uncivilisierte Völkerschaften oder auch nur Völkerschaften, die eine andere Civilisation haben als wir. Dafür wird dasjenige, was durch die Vorgeschichte klargelegt wird, uns helfen die Entwicklung des Erkenntnisvermögens bei Kindern und die Sitten und Gebräuche der verschiedenen Völękerschaften verstehen zu lernen.

Man sieht also, dass sowohl Historiker und Archäologen von Fach, sowohl Naturforscher, die die Funde, als Sprachforscher, die die Worte ihrem Alter und ihrem Zusammenhange nach bestimmen, dass sowohl Pädagogen ja selbst Psychologen und Erkenntnistheoretiker, wie auch 
Ethnographen ihren Beitrag zu einen Studium der Vorgeschichte liefern müssen. Bezieht sich diese auf ein bestimmtes Fach, so muss der betreffende Fachmann selbst hinzutreten, um die innere Verbindung zwischen den zusammengebrachten Thatsachen herzustellen; alle oben genannten Forscher aber werden aus den vorhistorischen Studien Ausbeute für ihr eigenes Fach gewinnen.

Auch die Mathematik hat ihre Vorgeschichte, und diese gehört keineswegs $\mathrm{zu}$ den am wenigsten bedeutungsvollen. Bei der Beschränkung auf einen eng begrenzten und selbständig auftretenden Stoff kann sie $\mathrm{zu}$ verhältnismässig sichren und klaren Ergebnissen führen. Was man dabei über die Art und Weise erfährt, wie frühere Völkerschaften Grössen durch Zahlen und räumliche Darstellung behandelten, das liefert uns einen wesentlichen Beitrag zum Verståndnisse ihres Vermögens, sich die Erde unterthänig $\mathrm{zu}$ machen, und macht es uns dadurch leichter die übrigen Zeugnisse über ihr Leben und Treiben zu verwerten. Durch Klarstellung der Grundlage, auf der das Menschengeschlecht später eine besser geordnete Behandlung der Mathematik aufgebaut hat, liefert sie auch einen schätzenswerten Beitrag zur schärferen Erfassung der erkenntnistheoretischen Grundlage für die ersten und wichtigsten Begriffe dieser Wissenschaft.

Um die Vorgeschichte der Mathematik ans Licht zu ziehen, muss man bei den Sprachforschern Belehrung suchen über das Alter der Benennungen für die einfachsten Zahlen und über die in verschiedenen Sprachen angewandten Mittel, um Zahlen zu benennen, die nach Zehnern, Zwanzigern u. s. w, zusammengesetzt sind oder auf irgend eine andere Art, wie sie für Einteilung von Maassen oder Münzen (Zwölfersystem, Sechzehnersystem u. s. w.) benutzt sein konnte. Man muss auf Inschriften oder in alten Schriftdenkmälern erst die Bezeichnungen für 
einfache Zahlen suchen, die in früheren Zeiten meist in einer Marke oder einem Zeichen für jeden Einer bestehen, und darauf die mehr entwickelten Zeichen für zusammengesetzte Zahlen, die z. B. durch Wiederholung eines Zeichens für jede decimale Einheit, wie bei den Römern, gebildet sein können. Man muss die Spuren der Anwendung dieser Zeichen oder auch mechanischer Hülfsmittel zur Ausführung einfacher Rechnungen aufsuchen. Möglicherweise kann man dann auch in der Bilderschrift alter Zeiten Operationszeichen finden, wie wenn in ägyptischen Papyrushandschriften ein Vogelfuss durch die Seite, nach der er sich wendet, auf sehr deutliche Weise angiebt, ob eine Zahl «zu» oder «ab» zu zählen ist, also eine ähnliche Rolle spielt wie unsere Zeichen + und --.

Was nun die räumliche Anschauung betrifft, so ist die erste Abbildung, auf welche man trifft, ein Zeugnis für eine Vorstellung von Figuren, von denen die eine im Kleineren das ist, was die anderen im Grösseren, also von ähnlichen Figuren. Das Zeugnis ist um so beweisender, als die Perspektive damals nicht bekannt sein konnte, der Darsteller also, wenn auch oft nur mit wenig Glück, eine wirkliche Ähnlichkeit anstrebte. Dieses Streben muss bewusst gewesen sein, wenn die Abbildung dieselbe Anzahl von Dimensionen hat wie der darzustellende Gegenstand, wenn sie also entweder eine Skulptur ist, oder wenn ein durch seine Umrisse in der Ebene dargestellter Gegenstand selbst eben ist oder als eben betrachtet werden kann. Besonders gilt dies, wenn die Darstellung sich als ein Modell auffassen lässt, als eine Karte oder als den Grundriss eines Gebäudes, und um so mehr, wenn man sie als den Versuch zu einer geometrischen Figur betrachten kann. Da es klar ist, dass es bei der ersten Benutzung solcher Figuren, sei es bei der Entwicklung des Menschengeschlechts oder bei dem Unterricht unserer Kinder, gleichgültig hleibt, 
ob die Figur etwas kleiner oder etwas grösser gezeichnet wird, so wird hier, lange bevor eine klare Definition von ähnlichen Figuren gegeben werden kann, eine bewusste Anwendung von solchen gemacht.

Eine altägyptische Grabkammer, deren Dekoration unvollendet ist, zeigt wie diese Vorstellung sogar $\mathrm{zu}$ einer planmässigen Hervorbringung von Ähnlichkeit geführt hat. In dieser sieht man nämlich, dass man, um ein Bild nach einem neuen Maassstabe auf die Wand zu übertragen, cliese Wand und das Bild durch zwei Systeme von Parallelen in Quadrate geteilt und darauf in jedes Quadrat der Wand das eingetragen hat, was sich auf dem entsprechenden Quadrat der Vorlage befindet. Das angewandte Hülfsmittel besteht in Wirklichkeil in einer Anwendung rechtwinkeliger Koordinaten, die in ganzen Zahlen durch die benutzte Quadratseite als Einheit ausgedrückt sind; entsprechende Punkte werden als solche bestimmt, deren beide Koordinaten, jede für sich, in einem gegebenen Verhältnis stehen.

Bei der weiteren Durchforschung aufgefundener Abbildungen oder Dekorationen muss man besonders nach solchen Figuren suchen, die Bekanntschaft mit einigen einfachen geometrischen Konstruktionen verraten können oder wenigstens von einer geometrischen Auffassung der Figuren zeugen. Das Bestreben Senkrechten oder Parallelen zu zeichnen findet man sicher auf den kindlichsten Standpunkten; bei mehr entwickelten Völkern hat man solche Linien gewiss mechanisch konstruiert, anfangs vielleicht durch ebenso einfache Mittel wie wir sie benutzen, wenn wir gerade Linien, Parallelen oder Senkrechten durch gespannte Schnüre, durch Falten von Papier etc. herstellen. Eine vollkommenere Konstruktion dürfte wohl benutzt worden sein, wenn man solche Linien zu der oben erwähnten Übertragung eines Bildes in einem neuen Maass- 
stabe angewandt hat. Verzierungen, die auf irgend eine Weise regelmässige Sechsecke benutzen, beweisen die $\mathrm{Be}$ kanntschaft mit der einfachen Kolistruktion dieser Figur, zu deren Herstellung kein von einem Mechaniker hergestellter Zirkel notwendig ist. Dagegen wird man selbst bei ziemlich hoch entwickelten Völkerschaften vergebens nach der Benutzung regelmässiger Fünf- oder Zehnecke suchen. Diese sind aber auch erst durch ein ziemlich kompliciertes Verfahren herzustellen; nicht einmal auf den alten ägyptischen Monumenten hat man derartige Vielecke gefunden.

Eine keineswegs geringere Bedeutung als überlieferte Zeichnungen haben die erhaltenen Reste von Gebäuden. Sogar auf den frühesten Entwicklungsstufen wird man das Bestreben finden, dem Grundriss der Gebäude eine bestimmte Figur, wie Rechteck oder Kreis, zu geben, und bei den vollkommeneren Bauten, wie den ägyptischen Tempeln und Pyramiden, müssen die rechten Winkel durch Konstruktion hergestellt sein. Hieran kann man um so weniger zweifeln, als die Gebäude genau nach den Himmelsgegenden orientiert sind, so dass man auch verstanden haben muss die höchste Stellung der Sonne durch Konstruktion $\mathrm{zu}$ benutzen. Die Pyramidenbauten zeugen von der Bekanntschaft mit bestimmten geometrischen Figuren, und eine umsichtige Konstruktion ist erforderlich gewesen, um zu erreichen, dass die Pyramiden wirklich gerade standen und gegenseitig dieselbe Gestalt erhielten. Gleichgewicht hervorzubringen in so gewaltigen Bauten, wie die ägyptischen Tempel es sind, so dass sie bis auf die Gegenwart haben stehen können, und Obelisken zu transportiren und aufzurichten, hat auch eine recht bedeutende mechanische Einsicht verlangt.

Ich habe mich bestrebt hier klar zu legen, was man unter Vorgeschichte der Mathematik zu verstehen habe, 
und einige von den Mitteln anzugeben, wodurch man sie kennen lernen kann. Ich hoffe zugleich dadurch angedeutet zu haben, von welcher Bedeutung es sein kann sie zu studieren, aber die Zeit erlaubt es mir nicht, in diesen Vorlesungen genauer auf diese Untersuchungen einzugehnn. Ich muss nicht nur die vorhistorische Mathematik übergehen, sondern zum grössten Teile auch die vorwissenschaftliche Mathematik ${ }^{1}$ ). Darunter verstehe ich diejenige, die nur in der Zusammenfassung solcher Regeln besteht, die sich aus Versuchen oder durch zufällige Erfahrungen haben ergeben können - das letzte kann z. B. mit der Sechsteilung des Kreises der Fall gewesen sein - oder vielleicht auch in älteren Zeiten durch mehr exakte Untersuchungen, die nun verloren, also vorhistorisch sind. Von der vorwissenschaftlichen Mathematik werde ich nur soviel mitteilen, dass man dadurch eine Vorstellung gewinnen kann von dem im voraus bekannten Material, aus dem die wissenschaftliche Mathematik aufgeführt ist. Als Einleitung in die griechische Geometrie muss ich deshalb das anführen, was die Griechen, wie wir annehmen dürfen, von den Ägyptern und Babyloniern gelernt haben. Das Zahlenrechnen der Griechen werde ich dagegen im wesentlichen nur behandeln können als vorwissenschaftliche Einleitung zu der Rechenkunst, die entstand, als die Inder die nun gebräuchliche Schreibung der Zahlen durch Ziffern mit Positionswert erfanden. Denn einmal stand das Zahlenrechnen der Griechen auf alle

$\left.{ }^{1}\right)$ In Betreff der vorhistorischen sowohl als der vorwissenschaftlichen Mathematik kann ich auf P. la Cour, Historisk Mathematik, Et indledende Kursus, Kjøbenhavn 1888, verweisen. Nach dem Plane dieses Werks, welches zur Einführung in die ersten Elemente der Mathematik die Wege ihrer Entstehung benutzt, machen die beiden genannten Disciplinen einen wesentlichen Teil desselben aus. 
Fälle weit zurück hinter ihrem sonstigen mathematischen Vermögen, und zweitens können wir durch Anwendung ihrer Zahlzeichen und ihrer Hülfsmittel für das Rechnen, wenn sie auch von ihren eigenen grossen Mathematikern mit Erfolg haben benutzt werden können, nichts hervorbringen, was dasjenige überragt, was wir sonst zur vorwissenschaftlichen Mathematik rechnen. Indessen möge das doch mit dem Vorbehalt gesagt sein, dass diese Hülfsmittel des Rechnens sich vielleicht bei genauerer Untersuchung besser erweisen können, als sie uns zunächst vorkommen ${ }^{1}$ ); denn giebt es einen Fehler, der Schaden verursachen kann und verursacht hat nicht nur bei derartigen historischen, sondern auch bei ähnlichen ethnographischen Untersuchungen, so ist es der, den Wert des Gefundenen nur nach seiner grösseren und geringeren Ähnlichkeit mit dem zu messen, was ein Kulturmensch jetzt gebraucht, und das geringe zu achten, was der Kulturmensch, nur weil er es nicht kennt oder nicht versteht, nicht glaubt gebrauchen zu können.

\section{2. Ågypter und Babylonier.}

Was diese beiden Völkerschaften betrifft, so wollen wir, wie schon oben angegeben, nur kurz die mathematischen Kenntnisse und Fertigkeiten erwähnen, die sie besessen haben, als sie mit den Griechen in Berührung kamen, und die die Griechen von ihnen übernommen haben

1) Die sachverständigste Autorität auf dem hier berührten Gebiete, Paul Tannery, hat erklärt, dass er bei einem Versuche, sich praktisch in der Benutzung der griechischen Zahlzeichen zu üben, diese viel zweckmässiger gefunden habe als sie uns erscheinen, die wir von Kindheit an in der Benutzung eines anderen Zeichensystemes geübt sind. 
können. Um zunächst mit den Ägyptern zu beginnen, so weisen griechische Schriftsteller durchweg darauf hin, dass ihre eigenen ältesten Forscher die Ägypter zu Lehrmeistern gehabt haben, und berichten davon, wie ihnen die Gelehrsamkeit der ägyptischen Priesterkaste zugänglich gemacht worden sei. Als Veranlassung zu der Beschäftigung der Ägypter mit Geometrie wird hingewiesen auf die Überschwemmungen des Nils und die damit verbundenen Bestrebungen, jedermann hinterher den ihm gehörigen Grund und Boden genau wieder zukommen zu lassen. In jedem Falle ist es klar, dass der sehr hohe Wert der schmalen fruchtbaren Landstreifen zwischen der Wüste und dem Flusse zu einer genauen Landmessung auffordern musste. Wie grosse Bedeudung die ägyptischen Regeln für das Landmessen gehabt haben, geht auch daraus hervor, dass, nachdem die Griechen die Geometrie so hoch entwickelt hatten, es dennoch im wesentlichen die ägyptischen Regeln waren, die von den römischen Landmessern (Agrimensoren) benutzt wurden, denn diese verstanden die griechischen Begründungen sicher nur in sehr geringem Maasse. Als ein in manchen Beziehungen geschäftlich tüchtiges Kulturvolk und als ein bauendes Volk - wie wir bereits berührt haben - haben die Ägypter auch eine gewisse Rechenfertigkeit und andere geometrische Kentnisse nötig gehabt als diejenigen sind, die beim Landmessen gebraucht werden. Ein anderes Zeugnis für eine gewisse mathematische Einsicht ist ihre Astronomie, die an Bedeutung jedoch kaum der babylonischen gleichkam.

Was nun die Ägypter in späteren Zeiten wussten, das ergiebt sich teils aus dem, was die Griechen und später die Römer von ihnen erhalten haben, teils aus einzelnen direkt überlieferten Aufzeichnungen. Das scheint indessen nur sehr wenig von dem abgewichen $\mathrm{zu}$ haben, 
was man nach einer uralten Papyrushandschrift, dem sogenannten Rechenbuche des Königs Ahmes, bereits 1700 -2000 Jahre vor Christi Geburt wusste. Deshalb ist diese Sammlung von Aufgaben nebst ihren Lösungen die beste Quelle, um ägyptische Mathematik und Rechenkunst kennen zu lernen.

Indem wir nun diese und andere Quellen benutzen, wollen wir hier unserem Plane gemäss nicht darauf eingehen, wie die Ägypter ganze Zahlen dargestellt und mit ihnen gerechnet haben. Ausser diesen kannten sie Brüche und rechneten mit ihnen. Brüche werden gewöhnlich in Stammbrüche zerlegt, dass heist in solche mit dem Zähler 1. Das Rechenbuch des Ahmes enthält eine Tabelle über eine derartige Zerlegung von Quotienten mit dem Dividenden 2 und mit Divisoren von 3 bis 99; diese Tabelle schliesst mit $\frac{2}{99}=\frac{1}{66}+\frac{1}{19}$. Eine solche Zerlegung ist später auch von den Griechen benutzt worden, und wie wenig praktisch sie auch dem Anschein nach gewesen sein mag, so hat ihre Anwendung doch Einblick in die verschiedene Zusammensetzung ganzer Zahlen gewährt. Die Ägypter verstanden in der sogenannten »Hau»-Rechnung soche Aufgaben zu lösen, die in unserer mathematischen Sprache durch Gleichungen ersten Grades mit einer Unbekannten ausgedrückt werden:

$$
a x+b x+c x+\ldots=d,
$$

wo $a, b, c \ldots d$ aus ganzen Zahlen und Brücher bestehen, die aus Stammbrüchen zusammengesetzt sind; ferner behandelten sie solche Aufgaben, die unter die Gesellschaftsrechnung gehören, ja einzelne, die zu ihrer Lösung einfache arithmetische und geometrische Reihen erfordern. Bei der Lösung von Aufgaben, die, wenn man sie auf die Form einer Gleichung gebracht haben würde, von Gleichungen der oben stehenden Form abhängen würden, treffen wir zum ersten Male eine Anwendung der Methode 
des falschen Ansatzes, der sogenannten "Regula falsi», auf die wir später an vielen Stellen treffen werden. Sie besteht darin, dass man $x$ einen Versuchswert $x_{1}$ beilegt; liefert dieser durch Einsetzen $d_{1}$ statt $d$, so ist

$$
x=x_{1} \frac{d}{d_{1}} \text {. }
$$

In der Geometrie musste nach dem Gesagten die Bestimmung von Flächeninhalten zu den Hauptsachen gehören. Bei den Ägyptern wie bei mehreren anderen Völkern war es nicht ungewöhnlich, den Flächeninhalt eines Vierecks mit den Seiten $a, b, c$ und $d$ nach der unrichtigen Formel

$$
\frac{a+c}{2} \cdot \frac{b+d}{2}
$$

$\mathrm{zu}$ berechnen, und den Inhalt eines Dreiecks mit den Seiten $a, a$ und $b$ nach der darin enthaltenen Grenzformel

$$
a \cdot \frac{b}{2}
$$

aber diese Formeln liefern keine üble Annäherung, sobald die Winkel des Vierecks oder die Winkel an der Dreiecksseite $b$ sich nur wenig von einem Rechten unterscheiden. Der Ausdruck für den Inhalt liefert dann nur einen Fehler, den wir von zweiter Ordnung nennen. Wenn auch die Aufstellung dieser Formeln zur Anwendung auf andere Fälle hat verleiten können, so haben doch die Ägypter - so weit man aus der Grösse der Seiten in den vorgefundenen Beispielen mit einiger Sicherkeit schliessn kann - sie vorzugsweise da angewandt, wo sie eine gute Annäherung geben. Die Nachahmer der Ägypter, die römischen Landmesser, kamen dagegen auf die Idee, sie auf gleichseitige Dreiecke anzuwenden, obgleich sie bessere Berechnungsmethoden für diese besassen. Den Inhalt eines Kreises mit dem Durchmesser $d$ berechneten 
die Ägypter nach der Formel $\left(\frac{8}{9} d\right)^{2}$, was desselbe ist, als wenn man $\pi=\left(\frac{16}{9}\right)^{2}$, also gleich 3,16 setzt. Die benutzten Formeln zeigen, dass die Ägypter ebenso wie wir als Flächeneinheit das Quadrat mit der Längeneinheit als Seite benutzt haben. Die Konstruktion von rechten Winkeln im Felde scheint durch Konstruktion eines Dreiecks mit den Seiten 3, 4 und 5 geschehen zu sein. Bei der Konstruktion der Pyramiden, oder wenigstens bei der Bestimmung ihrer Dimensionen scheint man die Grösse des Verhältnisses zwischen der halben Diagonale der Grundfläche und einer Seitenkante benutzt zu haben, also das, was wir den cosinus des Winkels nennen, den die Seitenkante mit der Grundfläche bildet. Wenn übrigens noch Demokrit zu einer Zeit, wo die griechische Geometrie eine keineswegs geringe Entwickelung erfahren hatte, als Zeichen für seine eigene Fertigkeit in geometrischen Konstruktionen anführen kann, dass er darin nicht einmal übertroffen werde von den ägyptischen Harpedonapten (Seilspannern), d. h. von den Männern, die unter Beobachtung feierlicher Gebräuche dafür zu sorgen hatten, dass die Grundrisse der Tempel richtig zur Sonne lagen, so kann die Tüchtigkeit dieser Männer sich nicht auf die Anwendung so einfacher Konstruktionen, wie die von uns genannten es sind, beschränkt haben.

Während die Griechen namentlich von den Ägyptern Impulse erhielten zur Bildung der Geometrie, desjenigen Teiles der Mathematik, den sie zur grössten Vollkommenheit entwickelten, so waren die Babylonier ihre Lehrmeister in Astronomie und in der Ausführung der dazu gehörigen Berechnungen. Von daher schreibt sich die noch heute gebräuchliche Teilung des Kreises in Grade, Minuten und Sekunden nach dem Sexagesimalsystem. 
Die Teilung in $360^{\circ}\left(=2^{3} \cdot 3^{2} .5\right)$ hängt vielleicht damit zusammen, dass das Jahr ehemals zu 360 Tagen angenommen wurde. Die weitere sexagesimale Teilung kann teils hiervon abhängig sein, teils kann sie auf der Erkenntnis der Vorteile beruhen, die ein Zahlensystem gewähren muss, in dem die Grundzahl $2^{2} \cdot 3.5$, wegen ihrer Zusammensetzung aus den niedrigsten Primzahlen, einen sehr grossen Teil der niedrigeren Zahlen als Faktoren enthält. Zeugnisse für die konsequente Benutzung dieses Zahlensystemes hat man in Inschriften gefunden, die Tabellen enthalten über die Quadratzahlen bis $60^{2}$ und die Kubikzahlen bis $32^{3}$, geschrieben nach diesem System. Diese Inschriften sind einige Tausend Jahre alt.

Es ist auch wahrscheinlich, dass verschiedene von den Zahlenspekulationen, die mehrere Untersuchungen der Griechen über ganze Zahlen nach sich gezogen haben, ursprünglich der Zahlenmystik der Chaldäer und Baby. lonier ihren Ursprung verdanken. 


\section{Die griechische Mathematik.}

\section{Historischer Überblick.}

Da wir bei unserer Besprechung der griechischen Mathematik oft die Behandlung einzelner Gegenstände durch lange Zeiträume hindurch verfolgen müssen, so wird es nützlich sein, im voraus einen historischen Überblick zu geben, in dem wir teils die zeitliche Reihenfolge, in der die einzelnen Entwickelungsstufen hervortreten und die verschiedenen Mathematiker thätig waren, auseinandersetzen, teils die Verhältnisse beleuchten, unter denen die Mathematiker ihre Wirksamkeit übten.

Ein Mittelpunkt in der griechischen Mathematik wird von Euklid gebildet, der etwa 300 Jahre v. Chr. lebte. In seinen sogenannten «Elementen» besitzen wir ein Lehrbuch der Geometrie, das noch an einzelnen Stellen als solches benutzt wird, und das das elementar-geometrische Lehrgebäude enthält, dessen Hauptprincipien überall unter verschiedenen Formen dem Unterrichte noch heutigen Tages zu Grunde gelegt werden. In diesem Werke müssen wir auf der einen Seite Aufklärung über die zerstreuten Angaben suchen, die wir über die ältere griechişhe Mathematik besitzen; denn diese beziehen sich im wesentlichen auf die Entstehung der euklidischen Geometrie. Auf der anderen Seite muss dieses Werk die 
Grundlage für unser Verständnis der späteren Schriftsteller bilden; denn es war die Grundlage, auf der diese selber weiter bauten. Auch in äusserer historischer Beziehung bildet Euklid einen Mittelpunkt. Er war nämlich der erste grosse Mathematiker der sogenannten alexandrinischen Schule, und wirkte unter ganz anderen Umständen als seine Vorgänger.

Die voreuklidische Entwickelung der Mathematik erstreckt sich über die drei vorhergehenden Jahrhunderte und hat in jedem von diesen einen etwas verschiedenen Charakter.

Der erste griechische Mathematiker war Thales von Milet, der die Sonnenfinsternis vom 28sten Mai 585 vorhersagte. Hierzu muss er Regeln benutzt haben, die direkt von den Ägyptern herrührten und sich auf langjährige Beobachtungen dieser stützen. Von den Ägyptern stammen gewiss auch die meisten seiner mathematischen Kentnisse. Die Hauptsache ist jedoch, dass die Griechen mit ihm und der von ihm gestifteten philosophischen Schule, der sogenannten jonischen, anfingen nicht nur das mathematische Wissen zu sammeln, was sie von den Ägyptern erhalten konnten, sondern auch dies Wissen nach verschiedenen Richtungen hin $z u$ erweitern. Diese Arbeit des sechsten Jahrhunderts nahm ihren Anfang auf der kleinasiatischen Küste, aber durch die lebhaften Handelsverbindungen der Griechen wurde sie auch nach anderen Gegenden, wo die Griechen ansässig waren, verpflanzt.

Wir sehen deshalb auch an einem ganz anderen Orte, nämlich in Süditalien, den Hauptheerd für die Entwickelung der Mathematik im fünften Jahrhundert. In diesem hatte man erkannt, dass die nach und nach gesammelten und gefundenen mathematischen Wahrheiten auf sicheren Grundlagen aufzubauen seien, und gleich- 
zeitig mit der Entwickelung dieser benutzte man die mittlerweile sicher gestellten Resultate als Ausgangspunkte für neue und wichtige Erweiterungen.

Der Mann, dem wenigstens die Tradition den grössten Einfluss auf diese Arbeit zuschreibt, ist Pythagoras von Samos, den wir deshalb beim fünften Jahrhundert besprechen, wenn auch seine eigene Wirksamkeit wohl zum Teil vor das Jahr 500 fällt. In "Grossgriechenland», wie die damals reich blühenden griechischen Kolonien in Süditalien genannt wurden, stiftete er eine philosophische Schule, die sich stark nach aussen hin abschloss und sich, wie es scheint, durch mystische Ceremonien und Geheimhaltung ihrer Lehren in dieser Abgeschlossenheit zu erhalten suchte. Diese aristokratische Schule suchte sich auch politisch geltend zu machen, erregte aber den Unwillen aussen stehender und wurde gesprengt, als die Demokraten in Grossgriechenland die Gewalt an sich rissen. Da in weit späterer Zeit die sogenannten Neupythagoreer meinten, dass ihre Lehren, die zum Teil von religiös-ethischer Art waren, auf Pythagoras zurückzuführen seien, so umgaben sie diesen ihren vermeintlichen geistigen Vater mit so vielen legendenhaften Erzählungen, dass es schwierig ist, den wahren Kern darin zu finden. Was von diesen Erzählungen Interesse für uns haben kann, das sind die Berichte über seine Reisen nach Ägypten, wo er recht wohl gewesen sein kann, ebenso wie später Plato und Eudoxus, und der Bericht über eine sehr zweifelhafte Reise nach Babylon. Von Bedeutung für die Entwicklung der Mathematik ist die grosse Abgeschlossenheit dieser Schule gewesen, denn diese veranlasste das wirksame Zusammenarbeiten von Männern, die sich gegenseitig verstanden, trägt aber auch andererseits die Schuld dafür, das wir so wenig darüber wissen, was dem Meister gehört, und was den Schülern. 
Die Sprengung der Schule war die Veranlassung, dass wenigstens ihre mathematischen Lehren später über die verschiedenen Gegenden ausgebreitet wurden, in denen das griechische Volk sich niedergelassen hatte.

An anderen Orten haben diese Lehren sich aber gewiss vereinigt mit den Früchten der Arbeit, die von anderen auf philosophischem oder mathematischem Gebiete ausgeführt worden war. Es ist deshalb nicht leicht zu entscheiden, wie viel oder wie wenig von seiner Mathematik ein 'so selbständiger Denker wie der Philosoph Demokrit von Abdera (um 460 v. Chr.) von den Pythagoreern gelernt hat. Der etwas ältere Hippokrates von Chios, der sich, nachdem er Kaufmann gewesen war und sein Vermögen verloren hatte, in Athen aufhielt und dort Unterricht in der Mathematik erteilte, kann vielleicht verschiedenes von den Pythagoreern gelernt haben, gehörte aber keinenfalls ihrer Schule an. Er erhält eine besondere Bedeutung dadurch, dass wir ihm ein zusammenhängendes Stück Geometrie verdanken, die einzige derartige Probe, die aus dem 5ten Jahrhundert erhalten geblieben ist, und ferner dadurch, dass er in Athen wirkte, derjenigen Stadt, die schon damals im Begriffe stand der Mittelpunkt zu werden für das griechische Geistesleben, für griechische Kunst und Wissenschaft, und für den Kampf zwischen Sophisten - unter denen z. B. Hippias von Elis ein tüchtiger Mathematiker war - und Philosophen, und die im folgenden Jahrhundert auch der Hauptsitz für die mathematische Entwickelung wurde. In Süditalien nahm unterdessen die Entwickelung pythagoreischer Lehren ihren Fortgang, und ein bedeutender Mathematiker, Archytas von Tarent, den wir dort gerade am Schlusse des 5ten Jahrhunderts antreffen, wird ausdrücklich als der letzte bedeutende Pythagoreer bezeichnet; er lebte in 
seiner Geburtsstadt, wo er sowohl als Staatsmann, wie als Heerführer und Mathematiker angesehen war.

Durch Archytas ist die durch die alte pythagoreische Schule und ihre nächsten Nachfolger gewonnene Entwickelung weiter getragen $\mathrm{zu}$ denjenigen, die namentlich die mathematische Arbeit des 4ten Jahrhunderts leiten sollten, nämlich zu Plato von Athen und Eudoxus von Knidos, denn beide wurden auf ihren Studienreisen nach Süditalien mit Archytas bekannt und wurden von ihm beeinflusst. Bevor ich auf diese beiden Männer und ihre Schulen genauer eingehe, will ich, im Anschluss an meine Bemerkungen über die beiden vorhergehenden Jahrhunderte, im allgemeinen über das 4te Jahrhundert bemerken, dass es damals klar geworden war, dass volle Genauigkeit erst erreicht werden könne durch Bildung eines zusammenhängenden Systems. Teils durch wiederholte Versuche solche Systeme zu bilden, teils durch Entwickelung der notwendigen Methoden und durch Erweiterung und Verbesserung des Stoffes brachte man die elementare Geometrie auf den Standpunkt, den wir bei Euklid finden. Gleichzeitig hatte man begonnen eine höhere Geometrie zu entwickeln, in der die Lehre von den Kegelschnitten zur grössten Bedeutung gelangte.

Plato (429-348) ist der bekannte grosse Philosoph, der Schüler des Sokrates und Stifter der Schule, die nach der Stelle in Athen, wo sie sich um Plato schaarte, die Akademie genannt wurde. Plato's Interesse für die Mathematik schreibt sich nicht von Sokrates her, denn dieser wollte die Mathematik auf praktische Anwendungen beschränkt wissen. Aber Sokrates war auch nicht sein einziger Lehrer, und nach dessen Tode fand er Gelegenheit erst in Kyrene und dann in Süditalien sich in die Mathematik und Philosophie der Pythagoreer hineinzuversetzen. In Kyrene studierte er Mathematik bei dem- 
selben Lehrer wie ein anderer Athener, der bedeutende Mathematiker Theätet, nach dem er einen seiner Dialoge benannt hat; vielleicht waren er und Theätet gleichzeitig in Kyrene. In Sicilien schloss er Freundschaft mit Archytas; auch Ägypten besuchte er.

Wenn wir das richtig erfassen wollen, worin Plato's Einfluss auf die Entwickelung der Mathematik bestanden hat, so begegnen wir denselben Schwierigkeiten wie bei Pythagoras. Wie die Neu-Pythagoreer dem Pythagoras, so haben die Neu-Akademiker dem Plato in ihren Berichten gern die Ehre für das denkbar mögliche zuerteilen wollen. Selbst diese schreiben ihm jedoch nicht persönliche mathematische Untersuchungen von weiterer Bedeutung $\mathrm{zu}$, sondern zeigen sich vielmehr geneigt ihm die Ehre für die Methoden, die zu seiner Zeit in Gebrauch kamen, zuzuerkennen, und ihn den Ratgeber derjenigen sein zu lassen, die die eigentlichen mathematischen Fortschritte machten. Haben diese Angaben auch nicht viel Wahrscheinlichkeit für sich, so war es doch auf alle Fälle von der grössten Bedeutung für den Fortschritt der Mathematik, dass derjenige von den Schülern des Sokrates, der vor allen andern Träger der geistigen Entwickelung wurde und lernbegierige Menschen aus den Ländern und Kolonien der Griechen nach Athen zog, dass dieser grosses Interesse für die Mathematik und ihre weitere Entwickelung besass. Ist es auch nur eine Legende, dass er über den Eingang zur Akademie schreiben liess: $\mu \eta \delta \varepsilon i \varsigma ~ a ̉ \gamma \varepsilon o-$

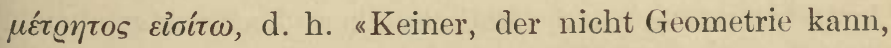
trete ein!», so geht doch aus seinen eigenen Arbeiten hervor, dass er eine gewisse geometrische Vorbildung als Voraussetzung für das Eindringen in die Philosophie betrachtete. So ist der Gebrauch, der in einem seiner Dialoge von den fünf regelmässigen Körpern gemacht 
wird, die Ursache gewesen, dass diese den Namen «platonische Körper» erhalten haben.

Auch der nächste grosse Philosoph, Aristoteles, der sich viel mit Naturwissenschaften beschäftigt hat, legt Interesse für Mathematik an den Tag, ohne jedoch an irgend einer Stelle eine besonders hervortretende mathematische Einsicht zu verraten. Die Stellung beider Männer $\mathrm{zu}$ diesem Fache war derartig, dass die Mathematiker Platz finden konnten in den wissenschaftlichen Gesellschaften der damaligen Zeit, der akademischen Schule Plato's und der peripatetischen des Aristoteles, dass sie in diesen mit andern Forschern zusammen arbeiten und bei ihnen Verständnis finden konnten, und dass Mathematik und Philosophie gegenseitig Impulse geben und empfangen konnten, sowohl durch friedliche Verhandlungen, als auch durch Zwistigkeiten. Teils wurde die Mathematik dadurch ein Glied in der höheren griechischen Bildung, teils lässt die Gestalt, die sie eben in dieser Zeit gewann, aufs deutlichste erkennen, dass sie sich ausgebildet hat in Kreisen fein gebildeter und mit Rücksicht auf die Korrektheit des Ausdrucks anspruchsvoller Denker.

Von mehr direkter Bedeutung für die Entwickelung der Mathematik als diese berühmten philosophischen Schulen war jedoch eine mathematisch-naturwissenschaftliche Schule, die sich zu Plato's Zeit in der blühenden Handelsstadt Kyzikos am Marmorameere um den als Arzt, Astronom und Mathematiker hoch angesehenen Eudoxus von Knidos sammelte. Als junger Mann hatte er sowohl Süditalien als Ägypten besucht. An der letzten Stelle war zu damaliger Zeit für einen griechischen Mathematiker kaum noch etwas in der Geometrie zu lernen. Dagegen sind dem Eudoxus als Astronomen die uralten Beobachtungen der Ägypter sehr zu Statten gekommen. 
Diese hat er denn auch zu benutzen verstanden; ihm gebührt nämlich das Verdienst, die Astronomie allein auf Beobachtungen und geometrische Untersuchungen gegründet zu haben mit Ausschluss der Sterndeuterei und leerer Spekulationen. In Süditalien studierte er Medicin und Geometrie, die letzte namentlich bei Archytas.

Die Verbindung beider Stifter mit den Pythagoreern ist gewiss eine gute Vorbedingung gewesen für das Zusammenarbeiten der Schulen, die sich an Plato und Eudoxus anschlossen, ein Zusammenarbeiten, dass gelegentlich auch die Form von Streitigkeiten annahm. Der Verkehr zwischen den beiden Schulen in Athen und Kyzikos wurde nicht nur hergestellt auf den zufälligen Wegen, zu denen der lebhafte Handel zwischen den beiden Städten Veranlassung geben konnte, sondern Eudoxus hat Athen besucht zusammen mit seinen Schülern, die dann Platos Vorträge gehört haben; mehrere von ihnen sollen sich in der Philosophie an Plato angeschlossen haben. Die am meisten bekannten Schüler von Eudoxus waren die Brüder Menächmus und Dinostratus. Von diesen soll Menächmus in philosophischem Anschluss an Plato über den Staat geschrieben haben.

Welche Resultate nun in den drei hier besprochenen Jahrhunderten erreicht waren, und welche Formen sich für Beweise und Darstellung entwickelt hatten, das lässt sich recht gut erkennen aus dem, was beim Beginn der nun folgenden alexandrinischen Schule vorhanden war, ferner aus den Sätzen, die Plato in seinen Dialogen benutzt, und aus der Betrachtung der von Aristoteles aufgestellten logischen Formen. Das Material, worauf diese letzteren sich in der einfachsten und genauesten Weise haben anwenden lassen, ist nämlich die Mathematik selber. Sie haben sich dann sicher auch eben durch den Gebrauch entwickelt, den die Mathematiker davon machten, 
und sie haben einen Teil der Ausbeute enthalten, die die Philosophie aus dem hier geschilderten Zusammenarbeiten mit der Mathematik davongetragen hat. Wenn wir im Folgenden den Standpunkt, der erreicht worden war, nicht nur als vollendete Thatsache schildern, sondern zugleich auch darüber Rechenschaft ablegen, was den Einzelnen $\mathrm{zu}$ verdanken ist und wie sich die Ideen nach und nach mit einander verküpft haben, so stützen wir uns dabei teils auf die bei den späteren Schriftstellern zerstreuten Äusserungen hierüber, teils auf einen Geschichtsschreiber der Mathematik aus dem Schlusse der hier erwähnten Periode, den Peripatetiker Eudemus von Rhodus. Wir besitzen allerdings auch sein Werk nicht, wohl sind aber einzelne wichtige Auszüge daraus bei späteren Schriftstellern aufbewahrt worden. Durch ihn, also durch dritte Hand, sind wir mit dem oben èrwähnteñ Bruckstücke des Hippokrates von Chios bekannt geworden.

Es ist ein etwas unruhiges Bild, das die Betrachtung der verflossenen drei Jahrhundert in uns hervorgerufen hat. Die Mathematik nimmt ihren Anfang an der Küste Kleinasiens. Darauf lenkt ihre Entwickelung in Süditalien besonders unsere Aufmerksamkeit auf sich. Damn zieht Athen durch seine ganze geistige Überlegenheit auch die Mathematiker an sich. Sicher ist auch dauernd an anderen Orten gearbeitet worden, wie in Süditalien, wo anderthalb Jahrhunderte nach Ajchytas den Griechen ihr grösster Mathematiker in Archimedes erstehen sollte; aber aus der ganzen geistigen Oberherrschaft Athens folgte auch, dass die Arbeiten der in Athen lebenden Mathematiker am wenigsten vergessen wurden. Die grosse Verbreitung des mathematischen Studiums während der hier erwähnten Zeit hat ihren Grund teils in den lebhaften Handelsverbindungen, die zwischen den zerstreuten Griechen bestanden, teils in den vielen Kriegen und politischen 
Unruhen, durch die hervorragende Männer von einem Ort zum andern getrieben wurden. Unter ähnlichen Unruhen haben auch die gewirkt, die an derselben Stelle haben bleiben können, wie namentlich in Athen. Gleichzeitig werden die Mathematiker allerlei geistige Kämpfe zu bestehen gehabt haben, sowohl unter sich als auch mit Sophisten und Philosophen.

Unter solchen Umständen gewann die Mathematik nicht nur so grosse Verbreitung über manche Gegenden und $\mathrm{zu}$ allen wissenschaftlichen Forschern, sondern sie umgab sich auch mit dem sicheren Rüstzeug in Beweisführung und Darstellungsform, das wir heute noch so hoch bewundern. Ja in der Beweisführung können wir in der Regel nichts besseres thun als sie nachzuahmen; mit Bezug auf die Darstellungsform müssen wir jedoch sagen, dass die Sicherheit in dem Grade auf Kosten der Zugänglichkeit gewonnen war, dass sie späterhin mit Schuld daran wurde, dass der erreichte hohe Standpunkt nicht bewahrt werden konnte, als die mündliche Tradition verloren ging, und dass das volle Verständnis für den Tiefsinn der griechischen Mathematiker erst in der neueren Zeit hat wiedererworben werden können, wo die Mathematik, wenn auch immer in etwas von der griechischen Mathematik beeinflusst, sich allmählich wieder auf den von den Griechen behandelten Gebieten zu derselben Höhe erhoben hat.

Der erwähnte Niedergang der Mathematik begann jedoch keineswegs, wie es mit der Dichtkunst, der Beredsamkeit und anderen Künsten, sowie mit der Philosophie der Fall gewesen war, damals, als die äusseren Verhältnisse sich so wesentlich nach Alexanders der Grossen Tode änderten, im Gegenteil, damals trat die reichste Blüte der Mathematik ein. Wie bekannt übernahm bei der Teilung des Reiches Ptolemäus, der Sohn des Lagus, Ägypten mit der neu angelegten Stadt Alex- 
andria, und er und seine Nachfolger machten diese nicht nur zu dem wichtigsten Handelscentrum, sondern auch zu einem wissenschaftlichen Mittelpunkte ersten Ranges. Unter ihm und seinen nächsten Nachfolgern, die auch den Namen Ptolemäus führten, wurde das «Museum» gegründet, in dem gelehrte Männer ohne Sorge für ihren Unterhalt der Wissenschaft leben konnten. Die Ptolemäer gründeten und vermehrten die alexandrinische Bibliothek, in der nach und nach Abschriften von allen bedeutenden griechischen Arbeiten, die sich auftreiben liessen, gesammelt wurden. Wie an einer modernen Universität versammelten sich die wissbegierigen griechischen Jünglinge in Alexandria und nahmen Unterricht bei den dortigen Forschern, namentlich in Grammatik und Mathematik.

Diese Verhältnisse konnten nur nützlich sein für die Mathematik, die der Ruhe bedurfte, teils um die vielen gewonnenen aber zerstreuten Resultate in festen Systemen $\mathrm{zu}$ vereinigen, teils um die bereits gewonnenen fruchtbaren Methoden der Betrachtung dazu zu benutzen, sich auf noch grössere Höhen $\mathrm{zu}$ erheben als bisher erreicht waren. Ruhe war erforderlich für den fortgesetzten mündlichen Unterricht, denn ohne diesen würde die Forn, wodurch die Zuverlässigkeit gewährleistet wurde, grössere schriftliche Darstellungen nur wenig zugänglich gemacht haben. Da die Mathematik nunmehr zu einer selbständigen Wissenschaft herangewachsen war, so bedurfte man ferner Mathematiker von Fach, die nicht nötig hatten beständig mit den Philosophen über ihr Fach $\mathrm{zu}$ verhandeln und selbst Philosophie zu treiben. Eine derartige Verteilung der Fächer bei den alexandrinischen Gelehrten verhinderte jedoch nicht, dass ein und derselbe Mann in mehreren Fächern thätig war. Das war z. B. der Fall mit Eratosthenes von Kyrene, der in 
der letzten Hälfte des 3ten Jahrhunderts wirkte und eine Zeitlang der alexandrinischen Bibliothek vorstand. Ausser mit Philosophie und Sprachwissenschaft beschäftigte er sich mit Geographie - ja mit höherer Geodäsie, in sofern er die erste Gradmessung vornahm - sowie mit Chronologie und Mathematik. Die Ruhe, die den Mathematikern in Alexandria gegönnt wurde, mochte jedoch auch ihre Gefahren mit sich bringen, z. B. was Selbstvergötterung und Kameraderien betraf, und man darf es deshalb nicht beklagen, dass Archimedes, der grösste Mathematiker der alexandrinischen Zeit, nicht in Alexandria lebte, sondern in Syrakus. Von da aus sandte er nach und nach sowohl seine fertigen Arbeiten wie vorläufige Resultate nach Alexandiria, und da gewisse hier lebende Mathematiker sich einige der letzteren dadurch aneignen wollten, dass sie sie hinterher bewiesen, so führte er sie einmal dadurch an, dass er ihnen einige unrichtige Resultate übersandte - denn auch diese bewiesen sie. Archimedes' Aufenthalt ausserhalb Alexandria hat übrigens den für unsere Kenntnis seiner Werke nützlichen Umstand im Gefolge gehabt, dass er vieles schriftlich hat abfassen müssen, während er sich in Alexandria damit begnügt haben würde, es seinen Umgangsgenossen und Schülern mündlich mitzuteilen, oder es doch nur auf eine für diese bestimmte Form zu bringen.

Diejenigen Mathematiker aus dieser Periode, die uns bedeutende rein mathematische (geometrische) Werke hinterlassen haben, und die sicher auch die übrigen tüchtigen Mathematiker der damaligen Zeit bedeutend überragten, sind Euklid, etwa 300 v. Chr., Archimedes, † 212, und Apollonius, etwa 200 v. Chr.

Von Euklid, von dessen persönlichem Leben in Alexandria man nicht viel weiss, besitzen wir ausser seinen bereits genannten Elementen auch eine andere elementare 
Schrift, die gewöhnlich mit dem lateinischen Namen Data bezeichnet wird. Teilweise wenigstens kennt man eine Schrift über die Teilung der Figuren, eine astronomische Schrift, Phaenomena, und eine Optik, die die alleresten Elemente der Perspektive behandelt. Verloren sind seine vier Bücher über Kegelsehnitte und seine zwei Bücher über Oberflächenörter, ferner die Schrift über Porismen und eine Schrift über falsche Schlüsse. Auf den Inhalt dieser Schriften kann man schliessen aus späteren Sammlungen von Hülfssätzen und aus Kommentaren. So haben die Porismen mehrere von den Sätzen über Transversalen und Punktreihen enthalten, die jetzt in der projektivischen Geometrie behandelt werden.

Archimedes lebte in Syrakus, wo er ein sehr angesehener Mann war, ein Freund des Königs Hiero. Bei der Einnahme von Syrakus durch die Römer fand er seinen Tod, nachdem er sein mechanisches Wissen auf verschiedene Weise bei Verteidigung der Stadt angewandt hatte. Sicherlich hat er seinerzeit Alexandria besucht und Verbindungen mit den Männern angeknüpft, denen er später seine Schriften sandte. Von diesen sind folgende erhalten: Über Kugel und Cylinder; Messung des Kreises; Über Konoide 'und Sphaeroide; Über Spiralen; Über das Gleichgewicht ebener Figuren; Sandrechnung; Quadratur der Parabel und Über Körper, die auf einer Flüssigkeit schwimmen, die letzte jedoch nur in lateinischer Übersetzung. Durch spätere griechische Schriftsteller und durch arabische Überlieferung besitzen wir ferner einige Bruchstücke, von denen wir eine Arbeit über halbregelmässige Körper und eine Reihe geometrischer Sätze, "Die Archimedischen Hülfssätze», nennen wollen. Die "Hülfssätze» sind vielleicht jedoch zum Teil neueren Ursprungs.

Apollonius von Perga ist sicher in Alexandria 
thätig gewesen. Fon ihm sind sieben von den acht Bïchern erhalten, die er über die Kegelschnitte geschrieben hat; die vier ersten hat man auf griechisch, die drei folgenden kennt man durch eine arabische Übersetzung. Auf dieselbe Weise ist uns eine Schrift über den Verhältnisschnitt erhalten, während wir nur durch spätere Mitteilungen Kenntnis haben von den Schriften über den bestimmten Schnitt, den Flächenschnitt, über Berührungen und Einschiebungen. Apollonius scheint auch wesentlich zur Anwendung der Mathematik auf die Astronomie beigetragen $\mathrm{zu}$ haben.

Unter der Zeitgenossen, den nächsten Vorgängern und Nachfolgern der drei grossen Mathematiker wollen wir erwähnen: Aristäus, der etwas älter als Euklid war und verloren gegangene Arbeiten über räumliche Örter und reguläre Polyeder geschrieben hat, ferner den schon erwähnten Eratosthenes, Nikomedes, der zwischen Archimedes und Apollonius lebte, sowie Diokles, Perseus und Hypsikles. Der letzte hat ein Buch über reguläre Polyeder geschrieben, das in die gewöhnlichen Ausgaben des Euklid als 14tes Buch aufgenommen zu sein pflegt. Was die übrigen betrifft, so kennen wir von ihnen nur durch Erwähnung bei späteren Schiftstellern einige verlorene Schriften oder einzelne Resultate.

Auch nach der hier geschilderten Zeit treffen wir auf bedeutungsvolle Fortschritte in einzelnen Richtungen der griechischen Mathematik, und zwar zunächst in solchen, die Anwendung auf die Astronomie finden. Obgleich wir uns nicht auf eine Geschichte der Astronomie einlassen können, so wollen wir hier doch einen Überblick geben über diejenigen astronomischen Schriftsteller aus verschiedenen Zeiten des griechischen Altertums, deren 
Arbeiten eine solche Bedeutung für die Mathematik erhalten haben, dass wir sie genauer erwähnen müssen.

Wir haben bereits gesagt, dass Eudoxus, dem die Mathematik die tiefsinnigsten Methoden verdankt, auch eine griechische, wissenschaftliche Astronomie begründete. Diese erfuhr jedoch bald eine Beeinflussung von aussen her, denn der Eroberungszug Alexanders des Grossen machte die Griechen mit der alten chaldäischen Astronomie bekannt. Die grossen alexandrinischen Mathematiker waren, wie schon erwähnt, zugleich Astronomen. Zwischen diese, und zwar zwischen Euklid und Eratosthenes, haben wir Aristarch von Samos (etwa 270 v. Chr.) einzuschieben, der bereits diejenige Hypothese über das Weltsystem aufgestellt hatte, die anderthalb Jahrtausende später von Koppernikus bewiesen wurde. Die Arbeiten des Eratosthenes in der mathematischen Geographie mussten sich unter anderem als nützlich erweisen für die Reduktion der Beobachtungen, die von chaldäischen Astronomen an anderen Orten ausgeführt worden waren. Apollonius verdankt man gewiss nicht wenige der geometrischen Voraussetzungen, die den Fortschritten in Beobachtung und Berechnung zu Grunde lagen, die in der nachfolgenden Zeit von den griechischen Astronomen weiter entwickelt wurden. Seine nächsten Nachfolger waren wohl noch in Alexandria thätig, aber der Mann, der die höchste Stelle unter den griechischen Astronomen eingenommen hat, Hipparch von Nikäa (etwa 150 v. Chr.), beobachtete auf Rhodus. Er benutzte unter anderem die uralten chaldäischen Beobachtungen in ersehöpfender Weise, und ungefähr von seiner Zeit an hat sich morgenländischer Einfluss auch äusserlich gezeigt durch Teilung des Kreises in $360^{\circ}$ und durch allgemeine Benutzung des Sexagesimalsystemes bei astronomischen und trigonometrischen Berechnungen. Unter den späteren Astronomen ist in der 
Geschichte der Mathematik Menelaus von Alexandria aus der letzten Hälfte des ersten Jahrhunderts n. Chr. zu nennen, und namentlich der ein halbes Jahrhundert jüngere Ptolemäus, durch dessen «Grosse Zusammen-

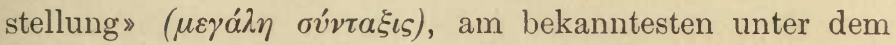
entstellten arabischen Namen Almagest, wir die griechische Astronomie (mit dem sogenannten ptolemäischen Weltsystem) und die damit verbundene Trigonometrie am vollständigsten kennen. Das meiste von dem, was sich bei ihm findet, rührt nämlich von älteren Astronomen her, besonders von Hipparch, von dem keine Schrift erhalten geblieben ist.

Während die Anwendung auf Astronomie auch nach der Zeit der grossen Mathematiker eine fördernde Rückwirkung auf die eigene fernere Entwickelung der Mathematik ausgeübt hat, so ist das nicht in irgendwie hervortretender Weise mit der Anwendung auf Landmessung und praktische Mechanik der Fall gewesen. Die theoretische Grundlage für das Landmessen war bereits in der griechischen Geometrie vorhanden, und das, was von theoretischer Mechanik aus dem Altertum herrührt, ist am klarsten und vollständigsten von Archimedes entwickelt worden. Dass dieser und seine Zeitgenossen zugleich bedeutende praktische Anwendung von der Mechanik gemacht haben, wissen wir aus späteren Berichten, und dass die griechische Geometrie von Anfang an Anwendung auf das Landmessen gefunden hat, das geht sowohl aus ihrem ägyptischen Ursprunge wie aus ihrem Namen hervor. Dieser bedeutet nämlich geradezu Landmessung, wenn auch das praktische Landmessen bereits zu Aristoteles' Zeiten den besonderen Namen Geodäsie führte. Zugleich wurde das Landmessen sowohl wie die Logistik oder Rechenkunst von den eigentlichen Geometern als unwissenschaftlich bei Seite gelassen. Des- 
halb wird in Euklids Elementen ebenso wenig Rücksicht genommen auf das Landmessen wie auf andere Anwendungen der Mathematik, die zu numerischen Bestimmungen führen.

Eine bedauerliche Folge hiervon ist die, dass wir keine direkte Kenntnis davon haben, wie man es in den besten Tagen der griechischen Mathematik verstand, die Ausbeute dieser Wissenschaft praktisch zu verwerten. Auskunft hierüber müssen wir, ausser bei den astronomischen, bei noch späteren Schriftstellern suchen. Unter diesen ist besonders Hero von Alexandrien zu nennen, dessen Lebenszeit man bis jetzt kurz nach der besten alexandrinischen Zeit gesetzt hatte, der aber nach den neuesten Forschungen frühestens im 2 ten Jahrhundert n. Chr. gelebt $\mathrm{zu}$ haben scheint. Seine Arbeiten, in denen richtige griechische Methoden und Formeln neben ägyptischen Näherungsformeln von verschiedenem Werte vorkommen, haben eine wichtige Rolle gespielt als Anleitung zum Feldmessen und zu anderen praktischen Anwendungen der Geometrie während der langen Zeiträume, wo man nicht verstand in die exakte griechische Geometrie einzudringen oder sie überhaupt nicht kannte. Was sie dafür namentlich geschickt macht, ist der Umstand, dass sie zahlreiche numerische Aufgaben behandeln. Eben hierauf beruht auch Hero's Bedeutung für die Geschichte der Mathematik, da er uns doch zu einer leidlichen Vorstellung davon verhilft, wie weit und auf welche Weise die Zahlenrechnungen, $\mathrm{zu}$ denen die wissenschaftlichen Resultate der griechischen Geometrie führen müssen, wirklich ausgeführt worden sind. Schade nur, dass man so wenig darüber weiss, in welchem Umfange die bei Hero vorgefundenen Methoden des Rechnens in Gebrauch gewesen sind, als die griechische Geometrie sich auf ihrer höchsten Höhe befand, und in welchem Umfange sie erst 
durch die auch nach jener Zeit dauernd von ihnen gemachte Anwendung entwickelt worden sind.

Die Entwickelung derjenigen Seiten der griechischen Mathematik, denen sie ihre eigentliche Grösse verdankt, war indessen schon vor dem Beginn unserer Zeitrechnung zum Stillstand gekommen. Um die Gründe hierfür zu verstehen, die in der eigenen Beschaffenheit der griechischen Mathematik lagen, müssen wir diese selbst zuerst kennen lernen. Hier können wir nur hervorheben, dass die günstigen äusseren Verhältnisse, unter denen im ersten Teile der alexandrinischen Periode gearbeitet worden war, aufgehört hatten. Die Gelehrten waren bereits weniger gut gestellt unter einzelnen der späteren Ptolemäer als unter den ersten Königen dieses Namens; aber die günstigen Verhältnisse hörten ganz auf, als die Römer in der Mitte des letzten Jahrhunderts v. Chr. Herren in Alexandria wurden, wie sie es an den meisten anderen Orten geworden waren, wo Griechen ansässig waren. In der Mathematik zeigten sie sich nämlich keineswegs als lernbegierige Schüler der Überwundenen. Im Laufe der Zeiten erlitt die alexandrinische Bibliothek, die dazu bestimmt gewesen war die gewonnene wissenschaftliche Ausbeute aufzubewahren, verschiedene Feuersbrünste. Wenn Alexandria nichtsdestoweniger fortfuhr der Ort zu sein, wo das Verständnis der alten Mathematik sich am besten erhielt, gelegentlich auch stärker aufleuchtete, so hängt das sicherlich damit zusammen, dass dies immer noch der Ort war, wo die meisten Arbeiten aufbewahrt wurden. So lebte dort Pappus am Ende des 3ten Jahrhunderts n. Chr.; er war gewiss kein grosser Mathematiker im Vergleich mit denen, die zu den Zeiten der Ptolemäer an derselben Stelle thätig gewesen waren, aber seine mathematischen Sammlungen sind von unschätzbarer Bedeutung geworden durch die Aufklärungen, die sie uns direkt oder 
indirekt durch Reihen von Hülfssätzen über jetzt verlorene Werke der grossen griechischen Mathematiker gebracht haben.

In einem einzelnen Zweige der Mathematik ist jedoch noch zu Pappus Zeit etwas Neues hervor gebracht worden, nämlich in der Arithmetik. Aus der Zeit zwischen den grossen Mathematikern und Pappus besitzen wir Arbeiten von mehreren arithmetischen Schriftstellern, unter denen namentlich Nikomachus (etwa 100 n. Chr.) grossen Ansehen genoss. $\mathrm{Er}$ hat eine erhaltene Einführung in die Arithmetik geschrieben. Dass er und einige andere arithmetische Schriftsteller uns etwas Neues haben bringen können, hat seinen Grund zunächst wohl darin, dass weitergehende arithmetische Untersuchungen nicht in den Rahmen der Arbeiten hineinpassten, die uns aus der besten Zeit aufbewahrt sind. Dagegen nehmen die Arbeiten, welche wir von Pappus' Zeitgenossen Diophant besitzen, eine derartige Sonderstellung ein, dass wir darin eine wirkliche Erweiterung der griechischen Mathematik erblicken müssen. Von seiner Hand ist uns das Meiste von einem grossen Werke über arithmetische Dinge erhalten; ob eine kleine Schrift über Polygonalzahlen einen Teil dieser grösseren ausgemacht hat, wissen wir nicht.

\section{Die pythagoreische Mathematik.}

Wenn wir uns nun dem mathematischen Inhalt der griechischen Geometrie zuwenden und mit der ältesten Zeit beginnen, so erfahren wir über das 6 te Jahrhundert nur ausserordentlich wenig. Allerdings schreibt Eudemus dem Thales verschiedene Sätze zu. Unter diesen kann er recht wohl den gekannt haben, dass der Peripheriewinkel auf dem Halbkreise ein Rechter ist, einerlei ob 
er ihn selbst gefunden oder von den Ägyptern erhalten hat; denn der Satz ergiebt sich ohne weiteres aus der leicht erkennbaren Thatsache, dass sich um ein Rechteck ein Kreis beschreiben lässt. Dagegen ist es schwierig irgend welchen Sinn darin zu finden, wenn Eudemus dem Thales den Satz zuschreibt, dass ein Kreis von einem Durchmesser halbiert wird, denn man kann kaum damit begonnen haben etwas so in die Augen fallendes zu beweisen. Eudemus kann jedoch gemeint haben, dass Thales notwendigerweise denselben Satz gekannt haben müsse, der zu Eudemus' eigenen Zeiten für notwendig angesehen wurde, um den Satz über Peripheriewinkel im Halbkreise $\mathrm{zu}$ beweisen. Auf ähnliche Weise kann es sich mit den gleichfalls von Eudemus genannten Sätzen verhalten, dass Scheitelwinkel oder Winkel an der Grundlinie eines gleichschenkeligen Dreiecks gleich gross sind, oder dass ein Dreieck durch eine Seite und die beiden anliegenden Winkel bestimmt ist. Der letzte Satz namentlich erhält erst seine Bedeutung als theoretischer Satz, wenn er in seiner natürlichen Verbindung mit anderen ähnlichen erscheint. Da über Thales' Bekanntschaft mit solchen nichts mitgeteilt wird, so hängt die Sache vielleicht damit zusammen, dass die Tradition dem Thales gewisse praktische Operationen beigelegt hat, $\mathrm{zu}$ deren theoretischer Begründung dieser Satz notwendig ist. Hierbei kann man denken an die dem Thales zugeschriebene Bestimmung des Abstandes von unzugänglichen Punkten oder Höhenmessung durch Schatten. Der Satz würde zunächst darauf hindeuten, dass diese Messungen mit Hülfe kongruenter Dreiecke ausgeführt worden sind. Die von den Ägyptern vorgenommene Bestimmung der Neigung einer Pyramidenkante deutet darauf hin, dass sie dazu ähnliche Dreiecke zu benutzen verstanden, also weiter waren als Thales; diesem fällt aber doch die Ehre zu, 
zuerst unter den Griechen mathematische Untersuchungen aufgenommen $\mathrm{zu}$ haben. Wieweit man ausserdem im 6ten Jahrhundert gekommen war, das sieht man am besten aus dem, worauf man im nächsten Jahrhundert weiter bauen konnte. Wenn die Pythagoreer beispielsweise die 5 regulären Polyeder entdeckten, so hat das jedenfalls mehr als unbedeutende, von ihren Vorgängern entwickelte, geometrische Voraussetzungen erfordert.

Weit mehr befriedigende Mitteilungen liegen vor über die Mathematik der Pythagoreer. Sind diese auch nicht nur unzuverlässig mit Bezug auf das, was dem Meister angehört und was den Schülem, sondern auch vielleicht geneigt den Pythagoreern vieles zuzuerkennen, was nur zu ihrer Zeit bekannt wurde, so geben sie doch demjenigen, der mit der späteren griechischen Mathematik vertraut ist, ein so zusammenhängendes, deutliches und verständliches Bild von ihrer ersten Entwicklungsstufe, von den Bestrebungen, die früh rege waren und später so deutliche Spuren in der griechischen, ja in der spätern Mathematik überhaupt, hinterlassen haben, dass sie es verdienen zusammengestellt zu werden. Dadurch wird man die Grundlage für die folgenden Arbeiten am Schlusse desselben Jahrhunderts erhalten und zugleich erreichen, deren Zweck richtig $\mathrm{zu}$ verstehen, und dabei wird auch die Gestalt, die man, namentlich im folgenden Jahrhundert, der Mathematik gab, ihre Erklärung finden.

Nach dem Bericht des Eudemus haben die Pythagoreer zunächst «die Geometrie zu einer wirklichen Wissenschaft erhoben, indem Pythagoras ihre Grundlage von einem höheren Gesichtspunkte aus betrachtete und ihre Lehrsätze mehr immateriell und intellektuell erforschte. Er hat ferner die irrationalen Grössen entdeckt und die Konstruktion der kosmischen Figuren (der regulären Polyeder),» $\mathrm{Zu}$ den specielleren Nachrichten, die 
wir anderen Schriftstellern verdanken, gehört - ausser einigen mehr philosophischen als mathematischen Definitionen von Punkt, Linie, Fläche und Körper - auch die, dass die Pythagoreer die Winkelsumme des Dreiecks kannten und die Einteilung der Ebene in (wahrscheinlich reguläre) Polygone, von denen um einen Punkt herum 6 Dreiecke oder 4 Vierecke oder 3 Sechsecke liegen konnten. Sie sollen die sogenannte Flächenanlegung erfunden haben, worunter man, wie wir sehen werden, die Auflösung quadratischer Gleichungen in geometrischer Form verstand, und die bekannte Konstruktion eines Polygons, das einem gegebenen gleich und einem anderen ähnlich ist. Von einem Pythagoreer wird erzählt, dass er sich des Verbrechens gegen die Schule schuldig machte, «den Satz von 12 Fünfecken in einer Kugel» zu verraten. Endlich kann angeführt werden, dass als pythagoreisches Zeichen das sogenannte Pentagramm angegeben wird, ein regelmässiges Sternfünfeck, dessen Seiten in dem umbeschriebenen Kreise Sehnen zu Bogen von der Grösse $\frac{4 \pi}{5}$ sind. Während einzelne Fälle des Satzes, der noch heutigen Tages der pythagoreische genannt wird, gewiss schon früher bekannt gewesen sind, wird der allgemeine Satz den Pythagoreern zugeschrieben; ferner eine von den Regeln, nach denen man rationale Zahlen für die Seiten eines rechtwinkeligen Dreiecks bilden kann, nämlich die Zahlen $a, \frac{a^{2}-1}{2}$ und $\frac{a^{2}+1}{2}$, worin $a$ eine ungerade Zahl bedeutet, während die Zahlen $a,\left(\frac{a}{2}\right)^{2}-1$ und $\left(\frac{a}{2}\right)^{2}+1$, worin $a$ eine gerade $\mathrm{Zahl}$ bedeutet, dem Plato zugeschrieben werden. Es wird berichtet, dass die Pythagoreer die drei Proportionen'kannten, nämlieh die arithmetische, die geometrische und die harmonische, ferner die Dreiecks- 
zahlen, d. h. die Summen der ersten Zahlen der natürlichen Zahlenreihe, und dass sie sich überhaupt mit Differenzreihen beschäftigten. Endlich wird berichtet, dass Pythagoras die Zahl zum Princip aller Dinge gemacht habe, und dass die Pythagoreer sich mit Untersuchungen über gewisse ganze Zahlen beschäftigt haben, wie «befreundete Zahlen», von denen die eine gleich der Summe der Faktoren der anderen ist, und "vollkommene Zahlen», die gleich der Summe ihrer eigenen Faktoren sind (wie $6=1+2+3)$. Endlich soll Pythagoras Arithmetik und Musik mit der Geometrie in Verbindung gebracht haben.

Wir werden ausführlicher über mehrere von diesen Gegeinständen und ihre Bedeutung für die griechische Mathematik sprechen, wollen aber erst kurz den Zusammenhang zwischen ihnen nachweisen und die gute Übereinstimmung zwischen den Angaben, die aus Quellen von verschiedenem Werte stammen.

Zunächst sei auf das Bestreben hingewiesen, die Begriffe Punkt, Linie u. s. w. klar von einander zu scheiden. Es ergiebt sich auch, dass man bereits im Besitze des Winkel begriffs war. Hiervon hat man Anwendung gemacht sowohl bei der Teilung der Ebene als bei der Untersuchung darüber, welche regulären Polyeder überhaupt möglich wären. Sicherlich war viel Arbeit zu leisten, bevor man zu einer so vollkommenen Bestimmung und Konstruktion auch vom Dodekaeder und Ikosaeder gelangte wie die ist, die wir bei Euklid finden; aber der erste Schritt hierzu, die Konstruktion des regelmässigen Fünfecks, war gemacht, und man war sichtlich stolz darauf, soweit gekommen zu sein. In der Konstruktion der Fünfecks- oder Zehnecksseite haben wir bereits dasjenige Beispiel für die geometrische Auflösung einer Gleichung zweiten Grades, welches die grösste Rolle bei 
Euklid spielt. Dass die Pythagoreer indessen bei diesem einen Falle nicht stehen geblieben sind, geht nicht nur aus der allgemeinen Erwähnung der Flächenanlegung hervor, sondern auch aus der besonderen Erwähnung des für diese Untersuchungen, wie wir sehen werden, so bedeutungvollen pythagoreischen Lehrsatzes und einer dafür eben so wichtigen Konstruktion. Daran hat sich denn auch die Entdeckung angeschlossen, dass Gleichungen zweiten Grades Veranlassung gaben zu inkommensurablen, numerische Gleichungen zu irrationalen Grössen (worunter wir beständig solche verstehen wollen, die mit der gebrauchten Einheit inkommensurabel sind). Wenn auch ein Teil ihrer zahlentheoretischen Untersuchungen eine Fortsetzung der Zahlenmystik der Babylonier gewesen sein mag, so ging doch ein anderer Teil darauf aus solche quadratische Gleichungen zu bilden, bei denen die Irrationalität vermieden wird.

Bei allgemeinen Untersuchungen lassen sich irrationale Grössen nun einmal nicht vermeiden. Dadurch wurden aber die bis dahin benutzten mathematischen Begründungen unzuverlässig, und es ist das grosse Verdienst der Pythagoreer hierauf aufmerksam geworden zu sein. Man kannte nämlich wohl Proportionen und hat sie wahrscheinlich in der einen oder anderen Form schon frïh angewandt; aber vor Eudoxus' Zeit konnte hierbei nur die Rede sein von Gleichheit der Verhältnisse zwischen ganzen Zahlen, oder von der Gleichheit solcher Verhältnisse mit den Verhältnissen zwischen geometrischen Grössen, die folglich kommensurabel sein mussten. Man benutzte die einfachen Rechnungsarten, z. B. die Multiplikation, und wusste edenso wie die Ägypter, dass z. B. ein Rechteck, wenn die Flächeneinheit das Quadrat über der Längeneinheit ist, gleich dem Produkte der Seiten wird; wenn aber die Seiten inkommensurabel sind, so wird nicht 
nur der Beweis durch Einteilung in Quadrate unbrauchbar, sondern der Satz selbst wird sinnlos, da es dem beim gewöhnlichen Rechnen gebildeten Begriffe eines Produktes widerstreitet, wenn die Faktoren irrationale Zahlen sind.

Diese Schwierigkeit war es, die die Pythagoreer und mit ihnen die folgenden griechischen Mathematiker überwanden durch geometrische Darstellung der allgemeinen Grösse. Im ersten Augenblick kann es allerdings so aussehen, als ob hiermit nur wenig gewonnen sei, da eine beliebig gezeichnete Strecke ebensowohl eine bestimmte Grösse hat wie eine beliebig gewählte Zahl. Die gezeichnete Figur dient jedoch nur dazu die Figur festzuhalten, die beschrieben wird, und in dieser können die Grössen alle die Werte annehmen, die zu der Beschreibung stimmen. Die Darstellung einer Grösse durch die Länge einer Strecke kamn dadurch, ebenso wie die in der Algebra gebräuchliche Darstellung durch einen Buchstaben, auf kontinuierlich variierende Grössen angewandt werden. Die Griechen wussten allerdings ebensowenig etwas von negativen wie von imaginären Grössen; aber das Bedürfnis nach den ersteren wird dadurch etwas verringert, dass die Variationen der Figur teilweise dieselben Verallgemeinerungen darbieten können, die wir nun durch Benutzung negativer Grössen erreichen.

Aus diesen Bemerkungen lässt sich erkennen, dass die Operationen mit den geometrisch dargestellten Grössen eine ähnliche Rolle spielen wie unsere algebraischen Operationen. Wir wollen deshalb die Lehre von diesen geometrischen Operationen die geometrische Algebra nemnen. Diese soll hier so dargestellt werden, wie wir sie kennen teils aus dem zweiten Buche von Euklids Elementen, teils aus der Anwendung, die überall von ihr in der griechischen Mathematik gemacht wird, namentlich 
da, wo man jetzt Gleichungen zweiten Grades benutzt. Sowohl bei Euklid als auch bei anderen liegt die geometrische Algebra so vielen Dingen zu Grunde, dass sich auch hieraus ein Beweis ergiebt für das hohe Alter, das wir ihr in Übereinstimmung mit dem Bericht über die Bekanntschaft der Pythagoreer mit der Flächenanlegung beilegen. Ihre Anwendbarkeit auf beliebige Grössen, irrationale sowohl wie rationale, und ihre hieraus folgende abstrakte Natur stimmt gut zu der Äusserung des Eudemus über Pythagoras' immaterielle Behandlung der Geometrie.

Es ist jedoch wohl möglich, dass diese abstrakte Natur nicht von vornherein so bewusst und ausgeprägt gewesen ist, wie sie es zu Eudemus' Zeit geworden war und bei Euklid ist. Im Gegenteil ist es natürlich und wohl übereinstimmend mit den Berichten über die Verknüpfung von Geometrie und Arithmetik durch die Pythagoreer, wenn man annimmt, dass die entsprechende geometrische Behandlung ganzer Zahlen, die bei Euklid zum Teil als eine Anwendung der allgemeineren geometrischen Algebra auftritt, vorangegangen ist. In den naheliegenden geometrischen Darstellungen von Eigenschaften ganzer Zahlen, mit denen man begonnen hat, hat man dann eine Darstellungsform gehabt, die von selbst ebenso leicht auf allgemeine kontinuierliche Grössen anwendbar war. Dessen ist man sich jedoch nur erst ganz allmählich bewusst geworden. Aus diesem Grunde wollen wir zuerst reden über die geometrische Arithmetik der Griechen als Einleitung zu ihrer geornetrischen Algebra. 


\section{Die geometrische Arithmetik.}

In unseren Lehrbüchern findet man durchgehends einen geometrischen Beweis für den Satz, dass in einem Produkte von ganzen Zahlen die Reihenfolge der Faktoren beliebig ist. Dieser besteht darin, dass man die Einheiten oder die Punkte, die diese darstellen, in Form eines Rechtecks hinschreibt. Jede horizontale Reihe enthält die Einheiten des Multiplikanden, und die Anzahl der Reihen ist der Multiplikator. Die Vertauschung der horizontalen Reihen mit den vertikalen ergiebt dann die Vertauschbarkeit der Faktoren. Benutzt man statt der Einheiten kleine Quadrate mit der Seite 1, so hat man gleichzeitig den geometrischen Satz bewiesen, dass die Grösse eines Rechtecks durch das Produkt der Seiten ausgedrückt wird. Unterlässt man dagegen eine bestimmte Einheit zu wählen, so erhält man, wenn die Seiten kommensurabel sind, den Satz, dass zwei Rechtecke sich wie die Produkte ihrer Seiten verhalten.

Von dieser Darstellung rührt die bei den Griechen allgemein gebräuchliche Benennung ebene Zahlen her für solehe, die aus zwei Faktoren zusammengesetzt sind, also eine rechteckige Fläche bilden, und die noch jetzt gebräuchliche Quadratzahl. Ebene Zahlen heissen ähnlich, wenn ihre Faktoren proportional sind; sie verhalten sich dann wie zwei Quadratzahlen.

Aus dem Quadrat, das eine gewisse Quadratzahl $\left(n^{2}\right)$ darstellt, erhält man die nächste $(n+1)^{2}$, indem man längs den beiden Seiten $2 n$ neue kleine Quadrate legt und noch eins in den dadurch entstehenden einspringenden Winkel. Diese ganze Ergänzungsfigur, sowie im allgemeinen eine Figur, die die Differenz zwischen zwei perspektivisch ähnlichen Figuren mit einem Eckpunkt als 
Ähnlichkeitspunkt darstellt, heisst ein Gnomon. Im vorliegenden Falle ist dieser $2 n+1$. Man findet auf diese Weise, dass die Quadratzahlen sich als Summen der ersten ungeraden Zahlen bilden lassen. Lässt man $2 n+1$ selbst eine Quadratzahl sein, so erhält man diejenige Bestimmung der rationalen Seiten eines rechtwinkeligen Dreiecks, oder diejenige Lösung der unbestimmten Gleichung

$$
x^{2}+y^{2}=z^{2}
$$

in ganzen Zahlen, die (S. 35) dem Py tha go r as zugeschrieben wurde. Diejenige, welche Plato zugeschrieben wurde, erhält man dadurch, dass man dem Gnomon die Breite 2 giebt.

Wenn man dem Gnomon eine beliebige Breite giebt, so erhält man die allgemeinste Lösung der eben genannten Gleichung in ganzen Zahlen. Um dies zu erreichen, benutzt Euklid im 1sten Hülfssatze zu Satz 28 des 10ten Buches eine Umformung, die in unserer algebraischen Sprache am nächsten einer Einführung der neuen Unbekannten $z+x=u, z-x=0$ (d. h. Breite des Gnomons $=v$ ) entsprechen würde; uv muss dann gleich einem Quadrat $y^{2}$ sein. Um uns enger an Eukliфd anzuschliessen, der sich in diesem Falle auf die in seinem 2ten Buche entwickelte geometrische

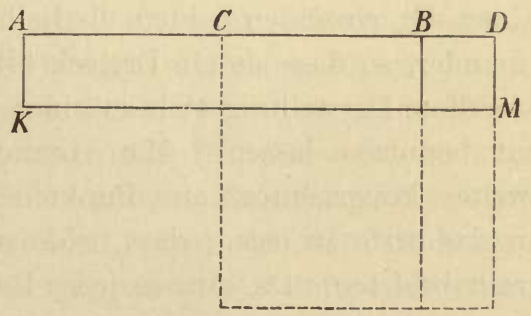
Algebra stützen kann, wollen wir aus dieser bereits hier den 6ten Satz des 2ten Buches, zu dem wir bald gelangen werden, anführen. Dieser sagt aus (vergl. im Folgenden S. 48), dass, wenn $C$ Mittelpunkt der Strecke $A B, D$ ein Punkt ihrer Verlängerung ist,

$$
A D \cdot B D=C D^{2}-C B^{2} \text {, }
$$

(d. h. mit den oben benutzten Zeichen: $u v=z^{2}-x^{2}$ ). 
Sollen hier alle Strecken ganze Zahlen darstellen, so müssen zunächst $A D(=u)$ und $B D(=\tau)$ beide entweder gerade oder beide ungerade Zahlen sein, damit $A B=2 C B$ (oder $u-v=2 x$ ) gerade werden kann. Die notwendige und ausreichende Bedingung dafür, dass der Gnomon $A D . B D$ eine Quadratzahl wird, ist demnächst die, dass $A D$ und $B D$ ähnliche Zahlen darstellen, oder in unserer Sprache, dass $A D=a n^{2}, E D=a n^{2}$, also $z=C D=a \frac{m^{2}+n^{2}}{2}, x=C B=a \frac{m^{2}-n^{2}}{2}, y=a . m n$.

Aus einer solchen Darstellung von Zahlen durch Rechtecke und Quadrate hat sich, wie wir sehen werden, die Grundlage für die geometrische Algebra entwickelt; aber die geometrische Arithmetik hat auch andere Figuren benutzt. Wir haben gesehen, dass den Pythagoreern die Kenntnis der Dreieckszahlen zugeschrieben wurde. Unter diesen versteht man Summen der ersten Zahlen der natürlichen Zahlenreihe, und zwar setzt man die Einer der einzelnen Zahlen als Reihen von Punkten untereinander, so dass sie ein Dreieck bilden. Man sieht leicht, wie diese Darstellung sich zu einer wirklichen Berechnung hat benutzen lassen. Man brauchte nämlich nur ein zweites kongruentes, aus Punkten gebildetes Dreieck so an das erste zu legen, dass beide zusammen ein Parallelogram bildeten. Da nun in jeder Reihe gleich viele Punkte liegen $(n+1$ bei $n$ Reihen ron Zahlen), so wird die Anzahl aller Punkte des Parallelogramms, also das Doppelte der Dreieckszahl, $n(n+1)$. Man sieht, dass dieses Verfahren ganz dasselbe ist wie das algebraische, bei dem man die arithmetische Reihe in umgekehrter Ordnung zu sich selbst addiert.

Da die Einheit, die in dieser Reihe die Differenz ist, willkürlich gewählt werden kann, und da ein konstanter 
Addend in jedem Gliede nur ein Produkt giebt, das zur Summe hinzugefügt werden soll, so hat man nun leicht eine beliebige arithmetische Reihe summieren können. Übrigens kann man die Differenz auch wie in der nebenstehenden Figur als eine Strecke abtragen, die unmittelbar eine beliebige Grösse bezeichnet. Aus einer weitergehenden Untersuchung von arithmetischen Reihen, die Archimedes in seiner Schrift über die Spiralen vornimmt, ergiebt sich, dass die Summation auf die angedeutete Weise vorgenommen worden ist.

Indem wir uns zurückwenden zur Darstellung der Einer durch Punkte, wollen wir noch ein, namentlich durch Nikomachus bekanntes, Mittel erwähnen zur geometrischen Darstellung arithmetischer Reihen mit dem Anfangsglied 1 und einer beliebigen ganzen Zahl $(n-2)$ als Differenz. Dieses besteht in der Anwendung der sogenannten Polygonalzahlen (n-eckszahlen). Man trägt das zweite Glied $(n-1)$ durch Punkte ab, die mit einem festen Punkte ein $n$-eck bilden. Für den festen Punkt als Ähnlichkeitspunkt wird dieses Vieleck zu einer Reihe ähnlicher $n$-ecke ergänzt durch eine Reihe von Gnomonen, von denen jeder ein Glied der Reihe darstellt. Für $n=4$ erhält man die Viereckszahlen, oder, da die Gestalt des Vierecks gleichgültig ist, die oben genannten Quadratzahlen.

Die hier erwähnte geometrische Arithmetik hat man auch auf den Raum ausgedehnt. Räumliche Zahlen sind solche, die durch ein Parallelepipedon dargestellt werden, also Produkte aus 3 Faktoren. Sind diese gleich gross, so erhält man Kubikzahlen. Bei zwei ähnlichen räumlichen Zahlen sind die Faktoren proportional; ihr Verhältnis ist also gleich dem zwischen zwei Kubikzahlen. 
Eine Pyramidalzahl ist die Summe einer Reihe von $n$-eckszahlen, anfangend mit 1 . Die Vielecke denkt man sich über einander gelegt, so dass sie eine Pyramide (einen Kugelhaufen) bilden.

\section{Die geometrische Algebra.}

Eine allgemeine, rationale oder irrationale Grösse lässt sich erstens durch die Länge einer (geradlinigen) Strecke darstellen. Addition und Subtraktion der in dieser Weise dargestellten Grössen findet statt durch Abtragen der einen Strecke auf der anderen oder auf ihrer Verlängerung. Ein Beispiel für die wirkliche Benutzung dieser Darstellung haben wir soeben gehabt in der Summation arithmetischer Reihen, die wir bei Archimedes kennen lernten. Sie lässt sich überhaupt benutzen zur Veranschaulichung von Gleichungen ersten Grades mit ganzen Koefficienten - oder nur mit rationalen, da sich diese zu ganzen machen lassen.

Multiplikation von zwei allgemeinen Grössen hat nach ihrer unmittelbaren Bedeutung keinen Sinn. Man half sich indessen dadurch, dass man die geometrische Darstellung eines Produktes von zwei ganzen Zahlen, die wir bereits kennen gelernt haben, auf allgemeine Grössen übertrug. Jedoch erweiterte man nicht, wie in der modernen Mathematik, die arithmetischen Begriffe Multiplikation und Produkt, sondern statt von einem Produkte der allgemeinen Grössen sprach man von dem Rechteck aus den beiden Strecken, die die Faktoren darstellen, und mit diesem Rechteck nahm man die Operationen vor. Als Anleitung für die Behandlung konnte man indessen, wegen der hiermit gleichartigen Darstellung von wirklichen Produkten ganzer Zahlen, beständig die 
arithmetische Behandlung dieser benutzen. Es kann deshalb nicht irreführend sein, wenn ich im Folgenden mit $a b$ und $a^{2}$ das Rechteck aus $a$ und $b$ und das Quadrat über $a$ bezeichne.

Auf diese Weise hat man eine zweite geometrische Darstellung von Grössen erhalten, nämlich als Flächen, vorläufig von Rechtecken und Quadraten. Um sie addieren und subtrahjeren zu können, musste man ihnen eine gemeinschaftliche Seite verschaffen, und zwar musste dies ohne Benutzung der Lehre von den Proportionen geschehen, denn diejenige, die man im 5ten Jahrhundert besass, war auf den ausschliesslichen Gebrauch von kommensurablen Grössen gegründet. Die Einführung einer neuen Seite in ein Rechteck wurde mit Hülfe des folgenden Satzes vorgenommen. Ein Rechteck wird durch Parallelen zu den Seiten, die durch einen Punkt derselhen Diagonale gehen, in vier Rechtecke geteilt, von denen die beiden, durch welche die Diagonale nicht geht, gleich sind (vergl. im Folgenden die Figuren auf S. 46-48, wo man sich dann im gegenwärtigen Zusammenhange die Quadrate mit Rechtecken vertauscht denken muss). Ist das eine von diesen das gegebene, so ist es leicht, dem anderen eine gegebene Seite zu geben. Diese Konstruktion, welche der Division entspricht, ebenso wie die Konstruktion eines Rechtecks aus gegebenen Seiten der Multiplikation, hat den besonderen Namen Flächenanlegung ( $\alpha \propto \varrho \alpha \beta o \lambda \eta ́)$ erhalten, oder einfache Flächenanlegung im Gegensatze zu der später folgenden elliptischen und hyperbolischen Flächenanlegung. Die hierfür benutzte Figur findet, wie wir bald sehen wer den, auch andere wichtige Anwendungen. Derjenige Teil der Figur, der aus den beiden gleich grossen Rechtecken und einem der beiden anderen besteht (z. B. CBEM in der Figur auf S. 47) heisst hier wie in der geometrischen Arithmetik Gnomon. 
Der hier angeführte Satz findet sich, und zwar auf die angegebene Weise angewandt, bei Euklid I, 43-44, wo er jedoch in einer etwas allgemeineren Form auftritt, insofern die Rechtecke mit Parallelogrammen von gleich grossen Winkeln vertauscht sind. In Euklids 2 tem Buche wird dagegen mit Rechtecken operiert. Eine Aufklärung über den Gebrauch, den man, gemäss den Mitteilungen über die Bekanntschaft der Pythagoreer mit Flächenanlegung, von den Sätzen dieses Buches lange vor Euklid gemacht haben muss, müssen wir indessen in seinem 6ten Buche suchen, wo die Anwendungen jedoch in einer verallgemeinerten Form vorkommen, die erst von Euklid oder seinen nächsten Vorgängern herührt ${ }^{1}$.

Ein Rechteck, dessen Seiten selbst Summen sind, wird die Summe aller der Rechtecke, die ein Glied in jeder der gegebenen Summen zu Seiten haben. An Stelle der modernen Formel

$$
(a+b)^{2}=a^{2}+b^{2}+2 a b
$$

trat die nebenstehende Figur (Euklid II, 4):

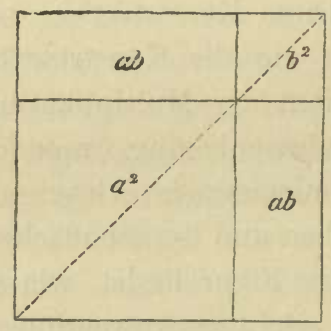

${ }^{1}$ Die Bedeutung der Verallgemeinerung wird in unserem 16ten Abschnitte erklärt werden. 
4. Die geometrische Algebra.

Die Aufgabe, die man jetzt durch die Gleichung

$$
a x-x^{2}=b^{2}
$$

darstellen würde, drückten die Alten folgendermassen aus: An eine gegebene Strecke $A B(=a)$ ein Rechteck $A M$ gleich einem gegebenen Quadrat $\left(b^{2}\right)$ so anzulegen, dass das (am Rechteck $a x$ über $A B$ ) fehlende Flächenstück ein Quadrat $\left(B M=x^{2}\right)$ wird. Diese Konstruktion, welche die elliptische Flächenanlegung (von der Mangel, das Auslassen) genannt wird, ergiebt sich,

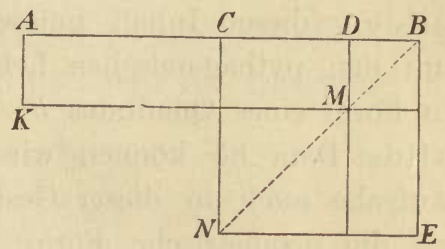
wenn man die Figur, durch welche die Aufgabe gelöst wird, auf die vorhergehende zurückführt. Ist nämlich $C$ die Mitte von $A B$, und legt man das Rechteck $C K$ an die Seite $D B$ (als $D E$ ), so sieht man, dass das Rechteck $A M$ gleich einem Gnomon wird, nämlich gleich der Differenz der Quadrate über $B C$ und $C D$, oder in der Sprache unserer Algebra, dass

$$
b^{2}=a x-x^{2}=\left(\frac{a}{2}\right)^{2}-\left(\frac{a}{2}-x\right)^{2} .
$$

Da nun $b$ und $C B=\frac{a}{2}$ bekannt sind, so kann man $C D=\frac{a}{2}-x$ durch den pythagoreischen Lehrsatz finden, und dadurch $x$.

Dass man die Aufgabe etwa in dieser Weise gelöst hat, lässt sich aus Euklid VI, 28 schliessen, wo sie jedoch in einer allgemeineren Form vorkommt. Die hier benutzte Umformung ist aber schon bewiesen in Euklid 
II, 5, wo gesagt wird, dass, wenn $C$ die Mitte und $D$ einen anderen Punkt von $A B$ bedeutet,

$$
A D \cdot D B+C D^{2}=C B^{2}
$$

ist.

Dieser Satz giebt unmittelbar die Lösung derselben, aber in anderer Form ausgedrückten, Aufgabe, nämlich: Teile eine gegebene Strecke $A B$ in zwei Abschnitte, die ein Rechteck von gegebenem Inhalt bilden (diesen Inhalt müssen wir uns jedoch vorläufig, um den pythagoreischen Lehrsatz anwenden $\mathrm{zu}$ können, in Form eines Quadrates $b^{2}$ gegeben denken). Aus Euklids Data 85 können wir sehen, dass die Alten die Aufgabe auch in dieser Gestalt gekannt haben, in der sie die geometrische Form für die Aufgabe ist, zwei Grössen zu bestimmen, deren Summe und Produkt gegeben ist. Der Übelstand, der mit der zuerst angeführten Darstellung der Aufgabe in Form einer elliptischen Flächenanlegung verbunden war, dass nämlich die Alten gewöhnlich nur die eine Lösung der Gleichung (1) mitteilen, fiel hierbei von selbst fort.

Auf ganz dieselbe Weise giebt Euklid in II, 6 eine (in VI, 29 einbegriffene) Lösung der Gleichung

$$
a x+x^{2}=b^{2} \text {, }
$$

die die Alten folgendermassen ausdrücken: An eine gegebene Strecke

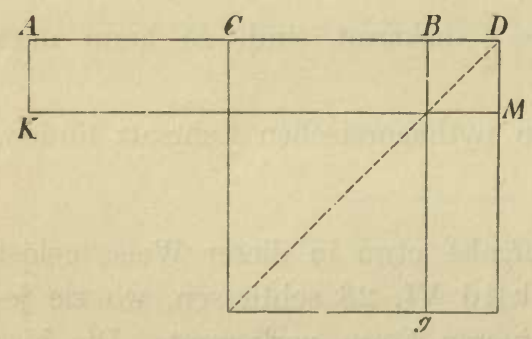
$A B(=a)$ ein Recht. eck $A M$ gleich einem gegebenen Quadrat $\left(b^{2}\right)$ so anzulegen, dass das (über das Rechteck $a x$ über $A B)$ überschiessende Flächen-

stück $B M$ ein Quadrat $\left(b^{2}\right)$ wird. Diese Konstruktion 
heisst die hyperbolische Flächenanlegung (von $v \pi \varepsilon \varrho-$ $\beta o \lambda \eta \dot{\eta}$, Überschuss). Ist die Aufgabe gelöst, und bedeutet $C$ die Mitte von $A B$, so wird das Rechteck $A M$ dadurch in einen Gnomon verwandelt, dass das Rechteck über $A C$ in die Lage $G M$ gebracht wird. Man findet hier, wo $D$ ein Punkt der Verlängerung von $A B$ ist, dass

$$
A D \cdot B D=C D^{2}-C B^{2} \text {. }
$$

Diese geometrische Umformung stimmt überein mit der algebraischen, durch die man jetzt die Gleichung (2) löst, nämlich

$$
b^{2}=a x+x^{2}=\left(\frac{a}{2}+x\right)^{2}-\left(\frac{a}{2}\right)^{2} .
$$

$C D\left(=\frac{a}{2}+x\right)$ lässt sich dann mit Hülfe des pythagoreischen Lehrsatzes bestimmen.

Euklids Satz II, 6 enthält unmittelbar die Lösung derselben Aufgabe in einer anderen Form, nämlich derjenigen, zwei Strecken $(A D$ und $B D)$ zu bestimmen, deren Differenz und deren Rechteck (gleich dem Quadrat $b^{2}$ ) gegeben sind, und diese ist wieder die geometrische Form für folgende: zwei Grössen zu bestimmen, deren Differenz und Produkt gegeben ist. Da die Aufgabe auch in dieser zweiten Form bei den Alten vorkam (vergl. Euklids Data 84), so ist es ohne Bedeutung, dass sich bei ihnen keine Form findet, deren Wortlaut ebenso unmittelbar die Gleichung

$$
x^{2}-a x=b^{2}
$$

wiedergiebt, wie die Flächenanlegungen die Gleichungen (1) und (2). Ob wir in unserer algebraischen Sprache die Gleichung (2) oder (3) erhalten, beruht nämlich nur 
darauf, ob wir in unserer modernen Umschreibung von Euklid II, 6

$$
B D=x \text { oder } A D=x
$$

setzen.

Wir sehen also, dass die Alten alle Formen der Gleichung zweiten Grades behandelt haben, die positive Wurzeln ergeben, und von anderen konnte nicht die Rede sein, da negative Grössen ein unbekannter Begriff waren (sonst hätten sich II, 5 und II, $6 \mathrm{zu}$ einem Satze vereinigen lassen). Wegen der hier mitgeteilten geometrischen Lösung setzten wir jedoch voraus, dass das gegebene Glied, das der Homogenität wegen in jedem Falle eine Fläche sein musste, in Form eines Quadrates gegeben wäre, und die Lösung wurde dann durch den sogenannten pythagoreischen Lehrsatz bewerkstelligt. Dieser, von welchem wenigstens spezielle Fälle den Ägyptern bereits bekannt waren, wird dem Pythagoras zugeschrieben, ohne dass man jedoch etwas darüber weiss, wie er ihn bewiesen hat. Der Beweis kann möglicherweise durch Benutzung ähnlicher Dreiecke geführt worden sein. kann also mit der damals bekannten Lehre von den Proportionen nur exakt gewesen sein, wenn die Seiten kommensurabel waren; denn die Einführung der allgemein gültigen geometrischen Begründungen hatte damals soeben begonnen, und der allgemein gültige Beweis bei Euklid (I, 47) soll, wie ausdrücklich bemerkt wird, von ihm selbst herrühren. Da Euklid beweist, dass das Quadrat über einer Kathete gleich dem Rechteck aus ihrer Projektion auf die Hypotenuse und der ganzen Hypotenuse ist, so liegt es nicht fern anzunehmen, dass für den älteren Beweis, den er ersetzen will, die ihm entsprechenden Sätze über mittlere Proportionalen benutzt worden sind.

Was dennächst die Verwandlung einer Figur in ein Quadrat betrifft, die entweder benutzt worden sein muss, 
um den Gleichungen die hier angenommene Form zu geben, oder um ohne Benutzung des pythagoreischen Lehrsatzes die Grösse zu konstruieren, die bei der modernen Lösung durch eine Quadratwurzel dargestellt wird, so wird den Pythagoreern ausdrücklich die Kenntnis der Aufgabe zugeschrieben: eine Figur zu konstruieren, die einer gegebenen gleich und einer zweiten ähnlich ist. Jedenfalls kann hierbei nur von geradlinigen Figuren die Rede gewesen sein, und in dem speciellen Falle, mit dem wir hier $\mathrm{zu}$ thun haben, ist die zweite Figur ein Quadrat. Die allgemeinere Form der Aufgabe ist diejenige, welche bei Euklid VI, 25 vorkommt, wo sie für Euklids verallgemeinerte Flächenanlegung benutzt werden soll. Wenn nun ein späterer Berichterstatter den Pythagoreern die Lösung der Aufgabe in dieser Form zugeschrieben hat, so hat er damit zu erkennen geben wollen, dass sie im Besitze der für Flächenanlegung notwendigen Voraussetzungen waren; für die einfache Flächenanlegung ist aber nur die Verwandlung in ein Quadrat erforderlich.

Die Verwandlung einer geradlinigen Figur in ein Rechteck hat keine besonderen Beschwerden verursacht. Wie man demnächst ein Rechteck hat in ein Quadrat verwandeln können ohne sich durch Benutzung des Begriffes mittlere Proportionale auf die vor Eudoxus unvollständige Lehre von den Proportionen zu stützen, das ersieht man aus Euklid, der sich in II, 14 nur auf die geometrische Algebra stüzt. Die Konstruktion beruht nämlich auf dem eben erwähnten Satze II, 5 (oder 6), nach dem ein Rechteck dargestellt wird als Differenz zwischen zwei Quadraten; danach lässt sich die Seite des dem Rechteck gleichen Quadrates mit Hülfe des pythagoreischen Lehrsatzes konstruieren. Die Umformung entspricht der Gleichung 


$$
x^{2}=a b=\left(\frac{a+b}{2}\right)^{2}-\left(\frac{a-b}{2}\right)^{2} .
$$

Diese Umformung enthält die Lösung der rein quadratischen Gleichung.

Eine bestimmte geometrische Anwendung der Flächenanlegung wird den Pythagoreern zugeschrieben, nämlich die Konstruktion der Seite des regelmässigen Fünfecks (und Zehnecks). Diese hängt bekanntlich ab von der Gleichung

$$
x^{2}=a(a-x)
$$

die sich umformen lässt in

$$
a^{2}=x^{2}+a x ;
$$

diese Gleichung wird durch hyperbolische Flächenanlegung gelöst. In II, 11 benutzt Euklid genau dieselbe Umformung der Gleichung in geometrischer Form für die Lösung der genannten Aufgabe.

Die Sätze II, 5 und 6 werden nicht ausschliesslich für die Auflösung von Gleichungen zweiten Grades benutzt. Wir haben bereits (S. 41) eine arithmetische Anwendung erwähnt, die Euklid davon im 10ten Buche macht, und soeben haben wir besprochen, wie sich die Benutzung der mittleren Proportionale auf diesem Wege vermeiden lässt. Ein anderes Beispiel dafür, dass die geometrische Algebra die Benutzung von Proportionen überflüssig macht, findet sich in Euklids Beweisen in III, 35-37 für die Sätze über die Potenz eines Punktes mit Bezug auf einen Kreis. Die Sätze II, 5 und 6 sagten aus (vergl. die Figuren S. 47,48 ), unter der Voraussetzung dass $C$ die Mitte von $A B$ und $D$ ein Punkt auf $A B$ oder ihrer Verlängerung ist, dass

$$
A D . D B= \pm\left(C B^{2}-C D^{2}\right) \text {. }
$$


Sind nun $A$ und $B$ die Schnittpunkte mit einem Kreise, dessen Mittelpunkt $O$, so erhält man durch den pythagoreischen Lehrsatz, dass

$$
C B^{2}-C D^{2}=O B^{2}-O D^{2},
$$

und damit sind die Potenzsätze bewiesen.

Die Elemente der geometrischen Algebra, die hier dargestellt sind, umfassen indessen namentlich die Behandlung der Gleichungen zweiten Grades, also gerade das Gebiet, auf dem sich wegen des Auftretens der irrationalen Grössen die Notwendigkeit einer anderen Darstellung als durch Zahlen fühlbar gemacht hatte. Bei dieser Behandlung konnte man sich mit der Benutzung von Rechtecken und Quadraten begnügen, sofern nicht bereits eine schon gegebene Grösse durch die Fläche einer anderen Figur dargestellt war. Während der weiteren Entwickelung der geometrischen Algebra und ihrer Anwendung - namentlich auf die Lehre von den Kegelschnitten - wurde sie jedoch so erweitert, dass auch andere Figuren zur Darstellung derjenigen Grössen dienten, Init denen operiert wurde. Es ist indessen klar, dass die geometrische Algebra ausschliesslich in ihrer Anwendung auf Rechtecke und - da man in der geometrischen Algebra niemals bestimmte Einheiten einführt, also mit homogenen Gleichungen operiert - Parallelogramme die geometrische Arithmetik mit einbegreift; denn nur dann können die Punkte, die in der Arithmetik die Einer darstellen, mit Quadraten von gleicher Grösse oder mit kongruenten Parallelogrammen vertauscht werden. Die Dreieckszahlen z. B. haben nichts mit dem Flächeninhalt des Dreiecks $\mathrm{zu}$ thun, ein Missverständnis, das später die römischen Landmesser dahin gebracht hat, die Formel $\frac{a(a+1)}{2}$ zur Berechnung des Inhaltes eines gleichseitigen Dreiecks mit der Seite $a$ zu benutzen. 


\section{Numerische quadratische Gleichungen; Ausziehen der Quadratwurzel.}

Aus der Übereinstimmung zwischen der geometrischen Algebra und der geometrischen Arithmetik, angewandt auf Rechtecke, geht hervor, dass es von vornherein sehr nahe gelegen hat, die gefundene allgemeine Lösung der quadratischen Gleichungen auf das Gebiet numerisch gegebener Gleichungen zu übertragen. Hier begegnete man indessen dem Übelstand, dass die Wurzeln im allgemeinen irrational wurden. Dass man nach Beispielen gesucht hat, bei denen dies vermieden wurde, ergiebt sich aus den Bestrebungen solche unbestimmte Gleichungen wie $x^{2}+y^{2}=z^{2}$ zu lösen. In den Aufgaben, welche die Geometrie oder andere Anwendungen darboten, musste man dagegen die Grössen so nehmen wie sie waren. Wenn man nun eine rationale Lösung, also eine solche, die sich durch Zahlen genau ausdrücken lässt, nicht finden konnte, so waren zwei Dinge zu thun. Einmal musste bewiesen werden, dass die gesuchten Grössen wirklich nicht rational wurden, und wenn man zu Gleichungen überging, bei denen die gegebenen Grössen bereits irrational waren, so galt es ferner die verschiedenen irrationalen Grössen, die vorkommen konnten, zu klassificieren; zweitens musste man der Anwendungen wegen auch die irrationalen Grössen mit möglichst grosser Annäherung ausrechnen.

Am weitesten brachten die alten Griechen es in der ersten der beiden genamnten Richtungen. Ein Beispiel für eine hierher gehörende Untersuchung haben wir bereits angeführt, nämlich Euklids Lösung der Gleichung $x^{2}+y^{2}=z^{2}$, und da er diese vollständig löste, so fand er nicht nur die ausreichenden sondern auch die not- 
wendigen Bedingungen dafür, dass $\sqrt{x^{2}+y^{2}}$ und $\sqrt{x^{2}-y^{2}}$ rational werden. Er fand also, dass sie irrational sind, wenn die Bedingungen nicht erfüllt werden. Von einfacherer Beschaffenheit ist ein wahrscheinlich sehr alter Nachweis dafür, dass $\sqrt{2}$ irrational ist; dieser hat wenn auch mit Unrecht - einen Platz am Ende des 10ten Buches in einigen Ausgaben von Euklid erhalten. Abgesehen von der geometrischen Darstellung lässt er sich etwa folgendermassen ausdrücken. Soll $\sqrt{2}=\frac{m}{n}$ sein, wo der Bruch $\frac{m}{n}$ so viel wie möglich verkürzt ist, so muss man $m^{2}=2 n^{2}$ haben. Hieraus folgt, dass $m^{2}$ eine gerade Zahl ist, also auch $m$. Da $\frac{m}{n}$ unverkürzbar ist, muss $n$ also ungerade sein. Wenn aber $m$ gerade ist, so folgt daraus, dass $m^{2}$ teilbar sein muss durch 4 , mithin $n^{2}$ durch 2 , folglich dass $n$ gerade ist. Da nun $n$ nicht zugleich gerade und ungerade sein kann, so kann $\sqrt{2}$ nicht ein unverkürzbarer Bruch sein.

Ein ähnliches Verfahren lässt sich wie bekannt im allgemeinen benutzen um nachzuweisen, dass eine Wurzel aus einer ganzen Zahl kein Bruch sein kann. Mehrere Sätze im 8ten Buche Euklids mögen ursprünglich mit diesem Ziel vor Augen entwickelt und in älterer Zeit auch dafür benutzt worden sein. Das gilt z. B. vom 6ten Satz, der - wenn auch in einer anderen Form -- ausdrückt, dass eine Potenz eines unverkürzbaren Bruches selbst ein unverkürzbarer Bruch sein muss. Indessen giebt es noch ein allgemeines Mittel, das Euklid im 10ten Buche giebt um die Rationalität einer Grösse zu prüfen oder, was dasselbe ist, die Kommensurabilität zweier Grössen. Dieses besteht in der Anwendung derselben Operation, die zur Bestimmung des grössten gemeinschaftlichen Maasses der 
beiden Grössen dient. Sind die Grössen durch Strecken dargestellt, so muss man die kleinere $b$ auf der grösseren so oft abtragen, dass der Rest $c$ kleiner wird als $b$, darauf $c$ auf $b$ u. s. w. Lässt diese Operation sich bis ins Unendliche fortsetzten, so sind die Grössen inkommensurabel. Auf diese Weise findet man leicht, dass eine Strecke durch stetige Teilung in Abschnitte geteilt wird, die inkommensurabel mil sich und der Strecke selbst sind. Heisst nämlich die Strecke $a$ und die Abschnitte $x$ und $y$, so hat man

$$
\frac{a}{x}=\frac{x}{y}=\frac{y}{x-y}
$$

Die Operation, die zum grössten gemeinschaftlichen Maass führen soll, wird also eine fortgesetzte Teilung der neuen Abschnitte, so dass sie sich offenbar nicht zu Ende führen lässt.

Da nun solche Wurzeln von Gleichungen zweiten Grades, die mit den gegebenen Grössen inkommensurabel werden, sich nicht durch diese und durch Zahlen ausdrücken lassen, so ist es begreiflich, dass die Griechen bei exakten Untersuchungen keine Näherungswerte einführten, sondern weiter operierten mit den gefundenen Grössen, die dargestellt wurden durch die Strecken, die sich aus der, der Iösung der Gleichung entsprechenden, Konstruktion ergaben. Es ist das ganz ebenso, wie wenn wir Wurzeln nicht ausrechnen, sondern uns damit begnügen diese durch Quadratwurzelzeichen und andere algebraische Zeichen auszudrücken. Da indessen eine Strecke wie die andere aussieht, so erhielt man dadurch nicht denselben Überblick, den die Zeichensprache uns gewährt. Deshalb wurde es notwendig eine Klassifikation der irrationalen Grössen vorzunehmen, die sich durch successive Lösung von Gleichungen zweiten Grades ergeben hatten. Eine 
5. Num. quadr. Gleichungen; Quadratwurzeln.

solche Klassifikation wurde zu Platos Zeit von Theätet vorgenommen, und seine Arbeit ist von Euklid fortgesetzt und ins 10te Buch der Elemente aufgenommen worden. Bei Besprechung dieses Buches werden wir darauf zurückkommen und wollen hier nur bemerken, dass diese Arbeit auch eine Prüfung der Fälle enthalten musste, in denen eine Grösse, die scheinbar einer Klasse angehört, sich in Wirklichkeit auf eine andere zurückführen lässt, also die Aufhebung doppelter Irrationalität.

Die Anwendungen dieser Klassifikation finden wir da, wo eine exakte Bestimmung von Grössen gewünscht wird, die von Quadratwurzeln abhängen oder überhaupt bei Gleichungen zweiten Grades gefunden werden. In der elementaren Geometrie gilt dieses von den Seiten regulärer Polygone oder den Kanten regulärer Polyeder. Mit dieser letzten Anwendung hat Theätet sich besonders beschäftigt und sie spielt eine Hauptrolle in Euklids Elementen.

In diesem Werk vermisst man dagegen jede genäherte numerische Berechnung. Das findet vielleicht seine Erklärung darin, dass man durch eine solche Berechnung die absolut exakte Bestimmung bei Seite lassen würde, die in der Geometrie beabsichtigt war; aber andererseits dürfte es auch damit zusammenhängen, dass den Griechen sowohl das Vermögen wie gute Hülfsmittel zu jeder wirklichen Berechnung fehlten, ein Mangel, der in verstärkter Weise hervortritt, wenn man über die vier einfachen Rechnungsarten hinausgehen muss, also schon bei der Berechnung der Quadratwurzel.

Indem wir uns vorläufig an das allgemeine Hülfsmittel halten, so wird sich die griechische Zahlbezeichnung (von der wir später Gelegenheit finden werden mehr zu sagen) doch wohl, wenn man sie so einübte, wie wir in unserer Kindheit in der unsrigen geübt werden, weit 
brauchbarer erweisen, als man sich von vornherein vorstellt. Bei Berechnungen hat man wohl auch solche mechanische Mittel wie eingeteilte Rechenbretter zu Hülfe genommen. Dass die Zahlbezeichnung nicht ausreichte, wenn es sich um die Darstellung grosser Zahlen handelte, sieht man jedoch daraus, dass damals, als die griechische Mathematik auf ihrer grössten Höhe stand, Archimedes und Apollonius - Männer, in deren Schriften ein wohl unterrichteter Mathematiker der Gegenwart ihm unbekannte Sätze und Beweise finden kann - besondere Systeme haben bilden müssen, um Zahlen von unbegrenzter Grösse bezeichnen zu können. Archimedes thut das in seiner Schrift über Sandrechnung, in der er eine Vorstellung von der Unendlichkeit der Zahlenreihe geben will und besonders berechnet, wie viele Sandkörner es in der ganzen Welt geben kann, wenn man dieser und den Sandkörnern gewisse Grössen beilegt. Es spricht auch keineswegs zu Gunsten der den Griechen selbst eigentümlichen Mittel der Zahlenberechnung, dass die griechischen Astronomen diese für nicht hinreichend entwickelt hielten, sondern zugleich mit der babylonischen Astronomie das Sexagesimalsystem der Babylonier für astronomische Rechnungen aufnahmen.

Was nun die Berechnung der Quadratwurzel bei den Griechen betrifft, so wollen wir zuerst eine besondere Bestimmung von $\sqrt{2}$ erwähnen, die man allerdings zunächst von einem späten arithmetischen Schriftsteller kemnt, die sich aber viel weiter zurückführen lässt, und deren Begründung sich bei Euklid II, 9 (und 10) findet. Diese Begründung ist zugleich ein Beispiel dafür, wie die geometrische Algebra angewandt wurde. Ist $C$ die Mitte und $D$ ein anderer Punkt der Strecke $A B$, so sagt Satz 9 aus, dass

$$
A D^{2}+D B^{2}=2 A C^{2}+2 C D^{2}
$$


Dieser Satz hätte durch Umlegungen von Rechtecken bewiesen werden können, aber bei Euklid ist er bewiesen mit Hülfe des pythagoreischen Lehrsatzes, angewandt auf gleichschenkelige rechtwinkelige Dreiecke. Das dürfte damit in Verbindung stehen, dass $\sqrt{2}$ eben als Hypotenuse $A B$ eines solchen Dreiecks $A E B$ dargestellt wird. Ist nun $Z$ der Punkt, in dem die Senkrechte auf $A B$ in $D$ die Kathete $E B$ schneidet, so wird $D B=D Z$ und

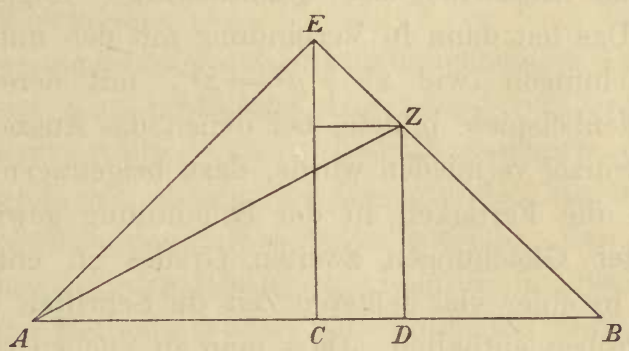

$A D^{2}+D Z^{2}=A E^{2}+E Z^{2}=2 A C^{2}+2 C D^{2}$.

Um die Anwendung der gefundenen Gleichung deutlicher $\mathrm{zu}$ zeigen, wollen wir

$$
C D=x, B D=y
$$

setzen, wodurch

$$
A D=2 x+y, A C=x+y \text {. }
$$

Bezeichnet man die letzten beiden Grössen mit $y_{1}$ und $x_{1}$, so hat man

$$
2 x_{1}^{2}-y_{1}{ }^{2}=-\left(2 x^{2}-y^{2}\right) .
$$

Die gefundene Gleichung dient dazu, aus einer Lösung von einer der beiden unbestimmten Gleichungen

$$
2 x^{2}-y^{2}= \pm 1
$$


in ganzen Zahlen eine Lösung der anderen in den grösseren Zahlen $x_{1}=x+y$ und $y_{1}=2 x+y$ abzuleiten. Fährt man auf diese Weise fort, so werden die Werte $\frac{y}{x}, \frac{y_{1}}{x_{1}}$ u. s. w., die abwechselnd zu klein und zu gross sind, sich $\sqrt{2}$ immer mehr nähern. Man kann ausgehen von $x=y=1$.

Auf ähnliche Weise kann man in anderen speciellen Fällen das Ausziehen der Quadratwurzel vorgenommen haben. Das hat dann in Verbindung mit den unbestimm. ten Gleichungen (wie $x^{2}+y^{2}=z^{2}$ ), mit deren Hülfe man Zahlenbeispiele bildete, bei denen das Ausziehen der Quadratwurzel vermieden wurde, dazu beigetragen bei den Griechen die Fertigkeit in der Behandlung gewisser unbestimmter Gleichungen zweiten Grades zu entwickeln, von der in einer viel späteren Zeit die Schriften des Diophant Proben enthalten. Dass man zu solchen speciellen Methoden seine Zuflucht genommen hat, zeugt dagegen nicht von einer irgendwie allgemeinen Fertigkeit im Ausziehen der Quadratwurzel. Von allgemeinen Hülfsmitteln hatte man jedoch erstens dasselbe zur Verfügung, das wir benutzen, nämlich den Ausdruck für $(a+b)^{2}$, dessen geometrische Form den Anwendungen kaum ferner lag als unsere algebraische. Zweitens führte eben das Verfahren, durch das man, wie wir gesehen haben, prüfte, ob eine Grösse irrational sei, geradeswegs zu einer Methode der Berechnung, die ungefähr mit einer Entwickelung in Kettenbrüche zusammenfällt.

Wie man aber verfahren ist, das lässt sich unmittelbar aus den Schriftstellern, bei denen sich genäherte Ausdrücke für Quadratwurzeln finden, nicht ersehen, da sie nur die Resultate anführen; aber man erhält doch eine Vorstellung davon, wie sehr oder richtiger wie wenig ent- 
5. Num. quadr. Gleichungen; Quadratwurzeln.

wickelt die Methoden waren. Sehr bezeichnend ist es, dass erst Archimedes eine Bestimmung von $\pi$, als zwischen den Grenzen $3 \frac{1}{7}$ und $3 \frac{1}{7} \frac{0}{1}$ liegend, durchgeführt hat. Aus dem Folgenden wird man nämlich erkennen, dass man vor ihm ohne grosse Mühe im Stande gewesen sein muss, die geometrischen Schwierigkeiten zu überwinden. Es ist also die numerische Berechnung und namentlich wohl die in dieser vorkommende Ausziehung von Quadratwurzeln, vor der man vor Archimedes zurückgeschreckt ist.

Unvermeidlich waren die entsprechenden Berechnungen, wenn man praktische Anwendung von Quadratwurzeln machen wollte. Es ist deshalb natürlich, dass man die meisten von diesen bei Hero findet, der mit praktischen Anwendungen vor Augen schrieb. Man findet so viele, dass es deutlich wird, dass er im Besitze einer wirklichen Methode gewesen ist. In seinen Bestimmungen ist der Grad der Genauigkeit jedoch nicht sehr gross im Verhältnis zu der allgemeinen theoretischen Einsicht, in deren Besitz man damals schon seit Jahrhunderten gewesen war. Der Umstand, dass er wirklich Quadratwurzeln auszieht, steht in Verbindung damit, dass wir zum ersten Male bei ihm Beispiele für eine durchgeführte Behandlung numerischer Gleichungen vom zweiten Grade finden. So sehen wir, dass, wenn das Glied $x^{2}$ einen Zahlenkoefficienten $a$ hat, er dieses in $a^{2} x^{2}$ verwandelte durch Multiplikation der Gleichung mit $a$, und demnächst $a x$ als Unbekannte betrachtete. Euklid hatte allerdings, wie wir sehen werden, auch derartige Gleichungen behandelt und überdies auf eine allgemein gültige Weise, die auch anwendbar bleibt, wenn der Koefficient $a$ irrational ist; aber gerade diese allgemein gültige Form der Behandlung zeigt nicht deutlich, wie man sich bei der praktischen Berechnung verhalten hat. 
Indem wir ganz zu Hero hinuntergegangen sind, haben wir jedoch die Berechnungen ausser Acht gelassen, die bereits vor seiner Zeit ausgeführt worden waren bei der Ausarbeitung der Sehnentafeln der astronomischen Schriftsteller. Diese waren in dem, in der Zwischenzeit von den Chaldäern eingeführten, Sexagesimalsystem ausgeführt. Da Hero hiervon keinen Gebrauch macht, so dürfen wir annehmen, dass sein Verfahren im wesentlichen das ursprünglich griechische war, das damals nur mehr entwickelt gewesen ist als zu der Zeit, mit der wir uns jetzt zunächst beschäftigen. Die Quadratwurzeln, die sich bei Ptolemäus finden, sind dagegen in sexagesimalen Einheiten etwa auf dieselbe Weise ausgerechnet, wie man jetzt Wurzeln in Decimalbrüchen berechnet. Es wurde vorhin berührt, dass die Griechen früher schon die theoretische Grundlage für eine solche Berechnung nach der Formel für $(a+b)^{2}$ besassen; diese konnte sie also naturgemäss bei dem grösseren Bedürfnis der Astronomie nach numerischer Genauigkeit zu einer wirklichen Ausführung solcher Rechnungen führen. Die schon erwähnten alten Tafeln über Quadrat- und Kubikzahlen im Sexigesimalsystem (S. 13) könnten jedoch wohl darauf hindeuten, dass das Wurzelausziehen in den babylonischen Ländern lange bekannt gewesen sein mag, und dass die Griechen auch in dieser Beziehung etwas von den östlichen Völkern haben lernen können, als das Sexagesimalsystem bei ihnen eingeführt wurde.

Wir werden kaum fehl gehen, wenn wir in der Entdeckung und der späteren Behandlung der irrationalen Grössen den Ausgangspunkt sehen für das, was sowohl die Hauptstärke, als auch die allerschwächste Seite der griechischen Mathematik ausmachte. Unter der beständig fortgesetzten Bemühung jeden Beweis auch auf diejenigen Grössen 
anwendbar zu machen, die sich nur näherungsweise durch Zahlen ausdrücken lassen und bei denen deshalb Zahlenbeweise ungenügend sein würden, entwickelten. sich die strengen Ansprüche an die Unfehlbarkeit der Schlüsse und die Genauigkeit des Ausdrucks, durch deren Erfüllung die Mathematik die exakte Wissenschaft geworden ist, und die Worte mathematische Gewissheit und absolute Gewissheit identisch geworden sind. Die Griechen haben dadurch den Grund gelegt, der erforderlich war um den hochragenden Gedankenbau des Archimedes und Apollonius zu tragen. $\mathrm{Zu}$ derselben Grundmauer hat die moderne Mathematik zurückkehren müssen, als sie nach langem Zwischenraume wieder ihre wissenschaftliche Bedeutung aufrichten sollte; ja $\mathrm{zu}$ den von den Griechen entwickelten und hochgehaltenen logischen Grundsätzen nimmt sie sogar in unseren Tagen ihre Zuflucht, um aufs neue der, jetzt auf arithmetischem Wege aufgeführten, Mathematik dieselbe Sicherheit zu geben wie die war, die die Griechen unter geometrischer Form erreichten, oder um die Infinitesimalrechnung unanfechtbar zu machen.

Eine so grossartige Leistung hätte keineswegs Gleichgültigkeit $\mathrm{zu}$ zeigen brauchen gegenüber den Versuchen dasjenige näherungsweise zu berechnen, was sich mit voller Genauigkeit nicht geben lässt. Archimedes zeigte, dass man auch die Resultate einer solchen Rechnung auf unanfechtbarer Weise aufstellen und beweisen kann, nämlich durch Angabe der Grenzen, zwischen denen die gesuchten Grössen liegen müssen, aber sein Beispiel wurde in den übrigen streng mathematischen Werken nicht befolgt. So kam es, dass die praktische Berechnung als etwas Untergeordnetes betrachtet wurde und nicht die verdiente Aufrnerksamkeit bei den eigentlichen Mathematikern fand, die doch am besten imstande gewesen wären die dahin 
gehörenden Methoden zu verbessern. $\mathrm{Zu}$ wie grossem Schaden es der Mathematik selbst gereichen sollte, sich in dieser Weise von den Anwendungen fern $\mathrm{zu}$ halten, das werden wir später kennen lernen.

\section{Das Unendliche.}

Es ist bekannt, dass Pythagoras die Zahl zum Princip aller Dinge machte, indem er sagte: «die Dinge sind Zahlen.» Da Zahl bei den Griechen ganze Zahlen, die Zahlen der natürlichen Zahlenreihe bedeutet, so stimmt dieser Ausspruch wohl ganz im allgemeinen zu den früher erwähnten Studien der Pythagoreer über die Lehre von den ganzen Zahlen und zu der mystischen Bedeutung, die sie gewissen Zahlenverbindungen beigelegt haben. Schwierig bleibt es jedoch, eine zu der Mathematik der Pythagoreer stimmende, ganz unmittelbare Bedeutung in den Wortlaut dieses Ausspruchs hineinzulegen. Eine solche Bedeutung müsste nämlich den späteren, mehr idealistischen Auslegungen vorangegangen sein. So wie sie da stehen, können die Worte kaum etwas anderes bedeuten, als dass alle Dinge sich durch Zahlen bestimmen lassen. Da hierbei nicht wohl von etwas anderem die Rede sein kann als von der Grösse der Dinge, so wird gesagt, dass diese sich durch Zahlen ausdrücken lasse. Das ist auch in der That der Fall mit kommensurablen Grössen, wenn man eine hinreichend kleine Einheit wählt. Es würde deshalb nicht weiter erstaunlich sein diesem Ausspruche $\mathrm{zu}$ begegnen - wenn nicht gerade die Pythagoreer entdeckt hätten, dass Grössen derselben Art nicht immer kommensurabel sind, dass also der angeführte Ausspruch seinem Wortlaute nach falsch ist. 
Deswegen braucht die hier versuchte Erklärung, die gewiss die einzige ist, die zu dem griechischen Gebrauche des Wortes Zahl stimmt, doch nicht unrichtig zu sein. Der Ausspruch kann älter sein als die Entdeckung der inkommensurablen Grössen, ja gerade das Bestreben, seine Anwendbarkeit zu zeigen, kann zur Entdeckung der inkommensurablen Grössen geführt haben. Eine philosophische Formel, an die man schon viele Betrachtungen angeknüpft hat, giebt man indessen nicht so leicht auf, selbst dann nicht, wenn sie sich in ihrer ursprünglichen Bedeutung als unrichtig erweist. Man modificiert diese Bedeutung derartig, dass sie fortdauernd anwendbar bleibt. Eine solche Modifikation konnte im gegenwärtigen Fall nicht so fern liegen. Wenn man die Inkommensurabilität von Grössen dadurch fand, dass die Rechnungen, die zur Bestimmung des grössten gemeinschaftlichen Maasses dienten, sich ins Unendliche fortsetzten, so lag es nahe zu behaupten, dass das grösste gemeinschaftliche Maass dann unendlich klein und unendlich viele Male in den Grössen enthalten sei. In diesem Falle wurden die Dinge bestimmt durch unendliche Zahlen oder durch unendliche Annäherungen, hervorgebracht durch Verhältnisse zwischen immer grösseren Zahlen.

Es würde jedoch kaum statthaft sein diese Erklärung aufzustellen, wenn es sich nicht nachweisen liesse, dass pythagoreische und andere Mathematiker zu ihrer Zeit wirklich auf ähnliche Weise Grössen durch unendliche Annäherung bestimmt hätten. Wir besitzen allerdings keine direkten Mitteilungen über solche Bestimmungen, aber ihre Existenz geht aus dem Kampf hervor, der gegen ihre Berechtigung geführt wurde, namentlich von Seiten einer anderen philosophischen Schule, nämlich der eleatischen. Ich meine hiermit die berühmten Sophismen, die von ihrem Stifter Zeno von Elea um die Mitte des 
Jahrhunderts aufgestellt wurden. Diese gehen überhaupt darauf aus die Ungereimtheiten zu zeigen, zu denen man gelangt, wenn man annimmt, dass die kontinuierlichen Grössen aus unendlich vielen unendlich kleinen Teilchen zusammengesetzt sind.

In zwei von den Sophismen wird bewiesen, dass Bewegung unmöglich sei. Der erste Beweis lautet folgendermassen. Um von einem Orte zum anderen $\mathrm{zu}$ gelangen muss man, bevor er erreicht wird, zuerst die Hälfte des Weges zurücklegen, dann die Hälfte von der Hälfte u. s. w. bis ins Unendliche. Die Bewegung verlangt also, dass unendlich viele Stücke des Weges durchlaufen werden, ist also - sagt Zeno - unmöglich.

Auch nicht - sagt Zeno in dem zweiten Sophisma kann der schnellfüssige Achilles die langsame Schildkröte einholen; denn er muss erst die Stelle erreichen, die die Schildkröte jetzt einnimmt, darauf den Weg durchlaufen, den die Schildkröte mittlerweile gekrochen ist u.s.w. bis ins Unendliche; aber auch diese Unendlichkeit zu erreichen ist unmöglich.

Da nun Zeno gewiss nicht die Realität der Bewegung bezweifelte, so ist seine Absicht eine andere gewesen, nämlich die, es als unmöglich hinzustellen, die kontinuierliche Bewegung durch eine solche Zerlegung in einzelne diskrete Momente zu beschreiben. Das, was er bekämpfen will, muss indessen von seinen Gegnern geltend gemacht worden sein. Was ist das nun? In seinem ersten Sophisma ist es die Richtigkeit der Behauptung, dass

$1=\frac{1}{2}+\left(\frac{1}{2}\right)^{2}+\left(\frac{1}{2}\right)^{3}+\ldots$ bis ins Unendliche.

In zweiten Sophisma ist es, wenn wir annehmen, dass Achilles sich $n$-mal so schnell bewegt wie die Schildkröte, die Behauptung, dass

$$
1+\frac{1}{n}+\frac{1}{n^{2}}+\ldots \text { bis ins Unendliche }
$$


einen endlichen Wert habe. Da man zu jener Zeit sicherlich auszurechnen verstand, wie lange Zeit Achilles wirklich gebrauchte um die Schildkröte zu erreichen, so haben Zenos Gegner auch gewusst, dass der endliche Wert der vorliegenden Summe von unendlich vielen Gliedern $\frac{n}{n-1}$

war. Diese positiven Resultate liegen so unmittelbar in den Betrachtungen, die Zeno als absurd angesehen haben will, dass man, wenn die Gegner nicht, wie ich annehme, vorher diese oder ähnliche aufgestellt haben, beinahe ihn selbst als ihren Entdecker ansehen müsste. Ohne mathematischen Sinn und ohne solche Einsicht verfällt man uämlich überhaupt nicht darauf, diese in mathematischer Hinsicht fruchtbaren Zerlegungen vorzunehmen. Wir sehen also, dass man in der Mitte des fünften Jahrhunderts der Frage nach der Summation einer unendlichen Quotientenreihe nicht fremd gegenüberstand, einer Summation, von der wir später Archimedes eine Anwendung unter grössere Sicherheit gewährenden Formen werden machen sehen.

Von einem streng logischen Standpunkt aus hat Zeno jedoch recht. Es kann nämlich nicht gestattet werden, unendliche Grössen zum Beweise positiver Resultate $\mathrm{zu}$ benutzen, solange das Unendliche nur durch seinen Namen erklärt ist, denn in diesem liegt nur das rein Negative, dass es unerreichbar ist. Innerhalb der griechischen Mathematik pflichtete man auch dem Zeno bei, und zwar in dem Grade, dass der Unendlichkeitsbegriff als positives Beweismittel im nächsten Jahrhundert ganz verdrängt oder auf eine Weise umgangen wurde, die gegen ähnliche Angriffe Sicherheit gewährte.

Sofort geschah das jedoch nicht. Die atomistische Schule, die die physischen Körper als aus unteilbaren Partikeln zusammengesetzt betrachtete, hat sich gewiss auch 
auf eine infinitesimale Untersuchung der geometrischen Zusammensetzung dieser Körper eingelassen, ja wahrscheinlich mit ihr begonnen. Das ist namentlich mit Demokrit, dem bedeutendsten Manne dieser Schule, der Fall gewesen. Es wird berichtet, dass er die Frage behandelt habe, ob zwei unendlich nahe liegende parallele ebene Schnitte eines Kegels als gleich oder ungleich gross betrachtet werden dürfen. Im letzteren Falle würde der Kegel treppenförmig aufgebaut sein, im ersteren würde er ein Cylinder sein. Diese Frage kann sich ganz natürlich erhoben haben, wenn man - wie in unseren elementaren Lehrbüchern bei Gelegenheit der Pyramide - durch ein integrationsartiges Verfahren das Volumen eines Kegels $z u$ berechnen oder auch nur Sätze über die Gleichheit von Kegeln $\mathrm{zu}$ beweisen versuchte. Auf die Beschäftigung mit infinitesimalen Fragen deuten vielleicht auch die Titel von mehreren seiner Schriften, die alle verloren sind, hin, nämlich «Über inkommensurable Strecken und Körper», «Über die Zahlen», und vielleicht auch der Titel «Über Berührung zwischen dem Kreise und der Kugel». Im übrigen weiss man nichts von seiner mathematischen Thätigkeit, vielleicht weil in der folgenden Zeit die Mathematik namentlich durch Platos Schule oder doch in Verbindung mit ihr gefördert wurde, und diese die Philosophie des Demokrit vollständig verwarf.

Hat nun auch die Thätigkeit des Demokrit den Begriff des Unendlichen soweit vertieft, dass die darauf gebauten Begründungen an Zuverlässigkeit gewannen, und hat sie vielleicht ferner dessen Verwendbarkeit für wirkliche mathematische Untersuchungen wie über den Inhalt des Kegels gezeigt, so hat dieser sich dadurch doch nicht als anerkanntes mathematisches Beweismittel zu behaupten vermocht. Mehr als Zenos Dialektik, die von einem überlegenem Gesichtspunkte darauf ausging das Unzurei- 
chende des Unendlichkeitsbegriffes nachzuweisen für die Begründung von Resultaten, die an und für sich nicht zweifelhaft sein konnten, haben dazu die mehr unfreiwilligen Schlüsse beigetragen, zu denen er sich gebrauchen liess. Als Beispiel hierfür lässt sich der Beweis des Sophisten Antiphon anführen, der darthun sollte, dass der Kreis sich quadrieren lässt, das heisst, dass man ein Quadrat konstruieren kann, das eben so gross ist wie ein gegebener Kreis. Dieser Beweis lässt sich - wenn wir uns im übrigen auf die Berichte seiner Gegner verlassen dürfen - kurz folgendermassen wiedergeben: In den Kreis lässt sich ein gleichseitiges Dreieck beschreiben und demnächst durch Halbieren der Bogen reguläre Polygone von zunehmender Seitenzahl. Durch Fortsetzung bis ins Unendliche fällt das Polygon mit dem Kreise zusammen. Nun lassen sich alle diese Polygone quadrieren, folglich auch der Kreis.

Durch solche Missbräuche wurde das Vertrauen zu infinitesimalen Betrachtungen in dem Grade erschüttert, dass, als endlich Eudoxus den Weg fand, auf dem sich die Richtigkeit hierher gehöriger Schlüsse vollständig beweisen lässt, dieser nicht mehr zur Aufstellung des Unendlichkeitsbegriffes benutzt wurde; vielmehr wurde dieser in dem sogenannten Exhaustionsbeweise umgangen. Über diesen, der zu der Zeit, die uns jetzt beschäftigt, noch nicht zur Verfügung stand - werde ich mich jedoch erst auslassen, wenn wir seine Anwendungen bei Euklid kennen lernen. Ebenso werde ich damit warten, die mit dem Exhaustionsbeweise nahe verwandte Proportionslehre des Eudoxus zu erklären, bis wir sie im 5ten Buche des Euklid antreffen. Hier will ich nur bemerken, dass diese unmittelbar auf inkommensurable Grössen ebenso anwendbar ist wie auf kommensurable, 
so dass Proportionen zwischen inkommensurablen Grössen nach der Zeit des Eudoxus dieselbe Gültigkeit haben wie Proportionen zwischen kommensurablen Grössen.

\section{Die Quadratur des Kreises.}

Von diesen mathematischen Principienfragen wenden wir uns nun $\mathrm{zu}$ einzelnen bestimmten Untersuchungen, die gleichfalls im 5ten Jahrhundert begonnen sind und die Mathematiker in der ganzen voreuklidischen Zeit, ja über diese hinaus, beschäftigt haben. Wir haben soeben die Quadratur des Kreises berührt. Hierbei kann man sowohl an die Aufgabe denken, mit passender Annäherung Inhalt und Umfang des Kreises zu berechnen, als auch an die andere, ein Quadrat zu konstruieren, das gleich der Fläche des Kreises ist, und damit zugleich eine Strecke, die gleich seinem Umfange ist. Nach dem Vorangegangenen wird man verstehen, dass die Lösung der letzten Aufgabe durch ihren exakten Charakter und dadurch, dass sie hinterher zur Berechnung benutzt werden konnte, als das erstrebenswerte Ziel erscheinen musste, dem gegenüber man bis zu Archimedes die beschwerlichen Rechnungen, die doch nur ein ungenaues Resultat lieferten, unterliess. Die Scheu vor solchen Rechnungen war es, die den Antiphon, wie wir bereits gesehen haben, dahinbrachte, die einbeschriebenen Polygone, die ein vortreffliches Mittel für die Berechnung abgeben würden, zu einer unhaltbaren Behauptung über die Lösung der Aufgabe durch Konstruktion zu missbrauchen. Das Mittel um eine obere Grenze für den Flächeninhalt zu berechnen, kannte man auch, nämlich umbeschriebene Polygone; aber dieses wurde auch zu Sophismen missbraucht, wie zu dem eines gewissen Bryson, der - wie erzählt wird - 
behauptete, dass man, um den Kreis zu quadrieren, nur nötig habe den Umfang eines neuen Polygons zwischen die Umfänge eines einbeschriebenen und des dazu gehörigen umbeschriebenen Polygons hineinzuzeichnen. Das neue Polygon würde nämlich ebenso wie der Kreis grösser als das einbeschriebene und kleiner als das umbeschriebene werden - folglich(!) gleich dem Kreise sein.

Neben Antiphons Behauptung beweist dieses Sophisma jedoch, dass man zu jener Zeit ein Auge dafür hatte, auf welchem Wege man in der That angenäherte Bestimmungen des Kreises erreichen und kontrolieren konnte. Ein ähnliches Verdienst kann man nicht solchen Lösungen zuerkennen, die darin bestanden eine Zahl zu finden, die zugleich Quadratzahl und eine sogenannte cyklische Zahl war, d. h. eine solche, deren Quadrat mit denselben Ziffern endigt wie die Zahl selbst. Man erkennt aus diesem groben Sophisma, dass der von Zeno eröffnete Kampf gegen die von den Mathematikern aufgestellten unrichtigen oder unvollständigen Ausdrücke für richtige Gedanken nicht nur die Mathematiker zwang, der exakten Form grössere Sorgfalt zuzuwenden, sondern umgekehrt auch die Sophisten, die nicht Mathematiker waren, lehrte die mathematischen Formen $\mathrm{zu}$ gebrauchen um Ungereimtheiten aufzustellen. Wenn dagegen Aristoteles und seine Kommentatoren, durch die wir diese Beispiele kennen, einen Mathematiker wie Hippokrates von Chios beschuldigen, dass er auf Grund eines ähnlichen Fehlschlusses behauptet habe den Kreis quadriert zu haben, so muss hier wohl eine Verwechslung stattgefunden haben zwischen dem, was Hippokrates erstrebt, und dem, was er wirklich erreicht $\mathrm{zu}$ haben behauptet hat. Diese Beschuldigung ist indessen die Veranlassung dazu gewesen, dass wir noch seine Untersuchungen kennen; diese haben 
nicht nur zu einem hübschen Ergebnis gefühìt, nämlich zu den ersten Quadraturen von Flächen, die von krummen Linien begrenzt sind, sondern sie sind zuglejeh ein vortreffliches Beispiel für das, was einem tüchtigen Geometer des 5ten Jahrhunderts zur Verfügung stand und wie er es zu gebrauchen wusste. Namentlich aus diesem Grunde wollen wir hier einen Auszug aus dem Bericht des Eudemus über seine Arbeiten geben.

Wie berichtet wird beweist Hippokrates zuerst, dass ähnliche Kreisabschnitte sich wie die Quadrate über den Durchmessern verhalten, und zwar soll er dies mit Hülfe des entsprechenden Satzes über zwei Kreise gemacht haben. Der bewiesene Satz wird demnächst benutzt um das "Möndchen» zu quadrieren, das von einem Halbkreise und einem Bogen von $90^{\circ}$ über dessen Durchmesser begrenzt wird. Es wird bewiesen, dass dies Möndchen gleich dem gleichschenkeligen rechtwinkeligen Dreieck ist, das sich in den Halbkreis beschreiben lässt. Darauf wird auf folgende Weise ein Möndchen konstruiert, dessen grösserer Bogen grösser als ein Halbkreis ist. Es wird zuerst ein Trapez konstruiert, von dem 3 Seiten jede gleich $a$ und die vierte gleich $a l^{\prime} \overline{3}$ ist ( $«$ in der Potenz dreimal so gross als die anderen», d. h. ihr Quadrat ist dreimal so gross als jedes der anderen); um dieses wird ein Kreis beschrieben, und das Möndchen wird abgeschnitten zwischen dem grösseren Bogen der Sehne $a \sqrt{3}$ und einem Bogen über derselben Sehne, der demjenigen über der Seite $a$ ähnlich ist. Es wird gezeigt, dass das Möndchen dem Trapeze gleich ist.

Hippokrates hat noch ein drittes Möndchen konstruiert, das sich quadrieren lässt. In dem Bericht über dieses werde ich, abgesehen von einzelnen modernen Umschreibungen (wie $r \sqrt{\frac{3}{2}}$ ), mit einer direkten Wiedergabe 
des Referates von Eudemus beginnen ${ }^{1}$ ). "Ein Kreis mit dem Durchmesser $A B(=2 r)$ und dem Mittelpunkt $K$ ist gegeben. Die $C D$ steht senkrecht auf der Mitte von $K B$. Zwischen diese Senkrechte und die Kreisperipherie wird eine Strecke $E Z$ von der Länge $r \sqrt{\frac{3}{2}}$ eingeschoben, deren Verlängerung durch $B$ geht. $E H$ wird parallel $A B$ gezogen. Man zieht $K E$ und $K Z$, von denen die letzte

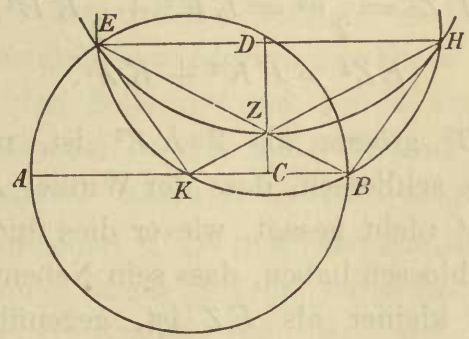

in ihrer Verlängerung die $E D$ in $H$ schneidet. Endlich ziehe man $B H$ und die Verlängerung $B Z$ von $E Z$. $B H$ wird gleich $E K$ sein, und um das Trapez $E K B H$ lässt sich ein Kreis beschreiben. Gleichfalls wird ein Kreisbogen durch $E, Z$ und $H$ gezogen. [Jeder von den beiden Abschnitten über den Strecken $E Z$ und $Z H$ wird den $\mathrm{Ab}$ schnitten über den Strecken $E K, K B$ und $B H$ gleich sein].

Das hier gebildete Möndchen $(E K B H Z)$ wird gleich der Figur sein, die man aus den drei Dreiecken (das ist Viereck $E K B H Z$ ) bildet...» Das wird dadurch gezeigt, dass jeder der beiden Abschnitte über $E Z$ und $Z H$ nach

1) So wie P. Tannery es in den Mémoires de la Socièté de Bordeaux t. V. (2. Reihe, 2. Heft) in einer von den Zusätzen des Simplicius befreiten Gestalt wiederzugeben versucht hat. Die eckigen Klammern bezeichnen eine von Tannery ausgefüllte Lakune. 
der Konstruktion das anderthalbtfache von jedem der drei Abschnitte über $E K, K B$ und $B H$ ist.

Hippokrates zeigt noch, dass der äussere Bogen $E K B H$ dieses Möndchens kleiner als ein Halbkreis ist, da der in das Segment $E K H$ einbeschriebene Winkel stumpf ist. Sein Beweis dafür lässt sich mit unseren Zeichen folgendermassen wiedergeben:

$$
\begin{gathered}
E Z^{2}=\frac{3}{2} r^{2}=E K^{2}+\frac{1}{2} K B^{2}, \\
E Z^{2}>E K^{2}+K Z^{2} .
\end{gathered}
$$

Dass $K B^{2}$ grösser als $2 K Z^{2}$ ist, muss Hippokrates daraus schliessen, dass der Winkel $K Z B$ stumpf ist; aber es ist nicht gesagt, wie er dies findet. Er kann es daraus geschlossen haben, dass sein Nebenwinkel $E Z K$, der $E K$, die kleiner als $E Z$ ist, gegenüberliegt, spitz sein muss.

In dem erhaltenen Schriftstück wird noch ein gewisses Möndchen konstruiert, das, $\mathrm{zu}$ einem gewissen Kreise hinzugefügt, eine Fläche liefert, die sich quadrieren lässt: Dieses Möndchen ist es, dessen Quadratur zur Quadratur des Kreises geführt haben würde. Dass dieses nicht identisch mit einem der vorher quadrierten ist, muss Hippokrates, der selbst imstande war diese Möndchen so herzustellen, dass sie sich quadrieren liessen, ebenso gut gesehen haben wie wir.

Um nun durch die citierten Untersuchungen in der That einen Einblick in die damaligen Leistungen der Mathematik zu gewähren, will ich zunächst darauf hinweisen, dass über eine Konstruktion, wie die eines Trapezes aus seinen Seiten, kein Wort verloren wird, dass die Benutzung der Grössen der Dreiecksseiten für die Untersuchung, ob ein Winkel im Dreieck spitz, recht oder stumpf ist, als wohl bekannt betrachtet wird, ebenso 
wie der Satz, dass Kreisflächen sich wie die Quadrate über den Durchmessern verhalten. Den euklidischen Beweis des letzten Satzes kann man jedoch noch nicht gekannt haben, überhaupt keinen, der den späteren griechischen Mathematikern genügt haben würde. Ausgangspunkte für einen faktisch richtigen Beweis kann man indessen in solchen Betrachtungen, wie die von Antiphon gemissbrauchten waren, gehabt haben. Eine Strecke wie $r \sqrt{\frac{3}{2}}$ hat man leicht konstruieren können, sei es nun durch die in der geometrischen Algebra erwähnte Methode, ein Rechteck mit den Seiten $r$ und $\frac{3}{2} r$ in ein Quadrat zu verwandeln, oder sei es durch Anwendung des pythagoreischen Lehrsatzes. "Die Einschiebung» der Strecke $E Z=r \sqrt{\frac{3}{2}}$ zwischen $C D$ und die Kreislinie, so dass ihre Verlängerung durch $B$ geht, ist abhängig von einer Gleichung zweiten Grades, die man, wie wir als ganz bestimmt annehmen, damals durch geometrische Konstruktion lösen konnte. Indessen ist es, wie wir bald erörtern werden, doch möglich, dass diese Konstruktion auf andere Weise ausgeführt worden ist.

Die verschiedenen Versuche, den Kreis mit Hülfe von Zirkel und Lineal zu quadrieren, misslangen, und in der neuesten Zeit hat man bewiesen, dass sie misslingen mussten. Das Verlangen nach einer exakten Lösung, die nach den Forderungen der damaligen Zeit durch Konstruktion zu einer geometrischen Darstellung führen sollte, konnte deshalb nur erfüllt werden durch Einführung anderer Kurven als Gerade und Kreis. Hierbei kam es nicht sonderlich darauf an, ob derartige Kurven sich mechanisch darstellen liessen, und noch weniger darauf, sie durch eine diskrete Reihe von Punkten darzustellen, denn diese würden ja nur eine Annäherung gestatten. Die Hauptsache war dagegen, hier wie in anderen ähnlichen 
Fällen, in einer exakten Definition eine mathematisch sichre, theoretische Grundlage für die Bestimmung $\mathrm{zu}$ geben, eine Grundlage, auf der sich eventuell weitergehende Untersuchungen, in denen die konstruierte Grösse verwendet wurde, aufbauen liessen. Man verfuhr in dieser Beziehung ebenso, wie wenn man in der Gegenwart neue Funktionen einführt für die exakte Bestimmung von solchen Grössen, die sich durch die bis dahin bekannten nur mit Annäherung darstellen lassen. Am besten war es natürlich, wenn eine und dieselbe Kurve sich auf verschiedene Konstruktionen anwenden liess, so dass die gemeinsame Theorie der Kurven allen Konstruktionen zu Gute kommen konnte.

Eben dies war der Fall mit einer Kurve, die für die Quadratur des Kreises benutzt wurde und deshalb den Namen Quadratrix erhielt. Sie soll ursprünglich von Hippias aus Elis erdacht sein, um für die Dreiteilung des Winkels benutzt zu werden. Die Eigenschaft, durch welche die Alten sie in Worten definierten, können wir, wenn wir mit $y$ die Ordinate eines ihrer Punkte in einem rechtwinkeligen Koordinatensystem bezeichnen, und durch $\vartheta$ den Winkel, den der Radiusvector desselben Punktes mit der Abscissenaxe bildet, darstellen durch die Gleichung

$$
\frac{y}{b}=\frac{\vartheta}{\varrho}
$$

wo wir durch $\varrho$ einen rechten Winkel bezeichnen, und durch $b$ den $\vartheta=\varrho$ entsprechenden Wert von $y$. Die Winkel werden durch die Bogen gemessen, die sie als Centriwinkel auf einem Kreise mit dem Radius $b$ abschneiden. Mit der jetzt gebräuchlichen Bezeichnung $\pi$ ist also $\varrho=b \frac{\pi}{2}$.

Da $y$ und $\vartheta$ proportional sind, so ist die Verwend. barkeit der Kurve für die Teilung eines Winkels in gleiche 
Teile oder in solche Teile, die in einem gegebenen Verhältnis stehen, ohne weiteres erkennbar. Ihre Verwend. barkeit für die Quadratur des Kreises wurde jedoch zuerst von Dinostratus entdeckt oder strenge bewiesen, da er bewies, dass die Abscisse ihres Schnittpunktes mit der Abscissenaxe gleich $\frac{b^{2}}{\varrho}$ oder $\frac{2 b}{\pi}$ sei; denn der Quotient $\frac{b^{2}}{\varrho}$ kann weder grösser noch kleiner sein als die genannte Abscisse. Wäre er grösser, so müsste es, da die Radienvectoren der Kurve mit $\vartheta$ wachsen, einen Punkt der Kurve geben, dessen Radiusvector gleich $\frac{b^{2}}{\varrho}$ wäre. Man müsste also (wenn wir der Übersichtlichkeit wegen unsere trigonometrischen Zeichen und Gleichungen da benutzen, wo Dinostratus Proportionen gebrauchte) haben

$$
\frac{b^{2}}{\varrho} \cdot \sin \vartheta=y=b \frac{\vartheta}{\varrho}=\frac{b^{2}}{\varrho} \cdot \frac{\vartheta}{b},
$$

oder der dem Radius $\frac{b^{2}}{\varrho}$ entsprechende Sinus müsste dem demselben Radius entsprechenden Bogen gleich sein. Wäre er kleiner, so müsste es einen Punkt geben, dessen Abscisse $\frac{b^{2}}{\varrho}$ wäre, für den also

$$
\frac{b^{2}}{\varrho} \operatorname{tg} \vartheta=y=\frac{b^{2}}{\varrho} \frac{\vartheta}{b},
$$

oder die dem Radius $\frac{b^{2}}{\varrho}$ entsprechende Tangente müsste dem demselben Radius entsprechenden Bogen gleich sein. Beide Dinge sind unmöglich.

Was nun den Inhalt dieses Beweises betrifft, so sieht man, dass Dinostratus sich nicht mit einer Bemerkung begnügt wie diejenige ist, die wir durch $\lim \cdot \frac{\operatorname{sinz}}{z}=1$ 
oder durch lim. $\frac{\operatorname{tg} z}{z}=1$ ausdrücken würden; vielmehr umgeht er ganz die Frage nach unendlicher Annäherung dadurch, dass er nur die Ungleichheiten $\sin z<z<\operatorname{tg} z$ benutzt, die ja im übrigen auch beide notwendig sind für eine exakte Bestimmung jeder der beiden Grenzwerte. Die Art, wie die Grenzbestimmungen umgangen werden, stimmt im wesentlichen mit der Art und Weise überein, wie dies im Exhaustionsbeweise geschieht; aber Dinostratus war auch ein Schüler von dessen Erfinder Eudoxus.

Wir werden später sehen, dass die Abhängigkeit zwischen Variationen von Kreisbogen und Strecken, die durch die Quadratrix dargestellt werden, einzelnen numerischen Bestimmungen $\mathrm{zu}$ Grunde gelegt wurde.

Auch Archimedes - dessen wirkliche Berechnung von Kreisen wir später erwähnen werden - hat Kurven untersucht, die sich etwa wie die Quadratix anwenden lassen, nämlich die sogenannten archimedischen Spiralen $(r=a \vartheta)$. Ihre Verwendbarkeit für Winkelteilung ist ohne weiteres erkennbar, und Archimedes schliesst sowohl die Bestimmung von Tangenten wie diejenige von Flächeninhalten an die Quadratur des Kreises an. Nach moderner Auffassung verwendet er wohl zunächst die Quadratur des Kreises oder die Zahl $\pi$ für diese Bestimmungen; aber der Vergleich mit der Benutzung der Quadratrix lässt erkennen, dass man ebensoviel Wert darauf gelegt hat auf diesem Wege, namentlich durch Bestimmung der Tangente, wenn auch nicht eine Konstruktion, so doch in Worten eine gute geometrische Bestimmung einer Strecke zu erhalten, die gleich der Peripherie des Kreises ist. Die Kurve selbst veranschaulicht auf die deutlichste Weise das periodische Wachsen von dem, was wir jetzt circuläre Funktionen (Kreisfunktionen) nennen. 


\section{Dreiteilung des Winkels; Einschiebungen.}

Wir haben soeben die Anwendung der Quadratrix und der archimedischen Spirale auf die Dreiteilung des Winkels berührt. Ausser diesen beiden will ich noch zwei andere Lösungen dieser Aufgabe anführen, die frühzeitig die Mathematiker beschäftigt haben. Die eine, deren Alter sich nicht bestimmen lässt, kann sehr wohl aus dem 5ten Jahrhundert herstammen, während die andere unter den von den Arabern aufbewahrten sogenannten archimedischen Hülfssätzen enthalten ist, vielleicht also von Archimedes herrührt. In beiden wird die Lösung auf eine sogenannte Einschiebung zurückgeführt.

1) Ist $A B C$ der Winkel, der in drei gleiche Teile geteilt werden soll, so zieht man zuerst $A C$ senkrecht auf $B C$, und $A E$ parallel

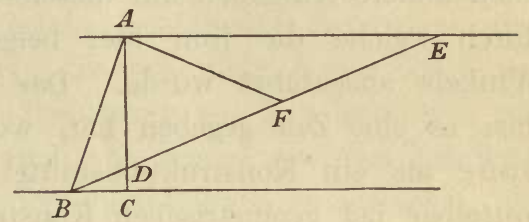
$B C$, und dann wird zwischen $A C$ und $A E$ die Strecke $D E=2 A B$ so eingeschoben, dass ihre Verlängerung durch $B$ geht. Ist dann nämlich $F$ die Mitte von $D E$, so ist

$\angle A B F=\angle A F B=2 \angle A E F=2 \angle C B D$, mithin

2) Ist $A B C$ der Winkel, der in drei gleiche Teile geteilt werden soll, und schneidet ein Kreis um $B$ die beiden Schenkel und die Verlängerung von $A B$ über $B$ hinaus in $A, C$ und $D$, so wird zwischen die Verlängerung von $B D$ und die Kreisperi- 
pherie eine Strecke $E F=B C$ so eingeschoben, dass ihre Verlängerung durch $C$ geht. Dann ist

$$
\angle D E F=\frac{1}{2} \angle B F C=\frac{1}{2} \angle F C B=\frac{1}{3} \angle A B C \text {. }
$$

Was nun die beiden hier verlangten Einschiebungen angeht, so sind sie wie die gestellte Aufgabe selbst von Gleichungen dritten Grades abhängig, lassen sich also nicht mit Hülfe von Gerade und Kreis lösen. Indessen sei hier bemerkt, dass die Zurückführung einer Konstruktion auf eine Einschiebung ohne genauere Angabe darüber, wie diese auszuführen sei, sehr oft in der griechischen Geometrie vorkommt. So haben wir eine solche in dem angeführten Bruchstück von Hippokrates getroffen, und Archimedes führt in seiner Schrift über Spiralen andere Aufgaben auf dieselbe Einschiebung zurück, durch welche die ihm hier beigelegte Dreiteilung des Winkels ausgeführt wurde. Das kann darauf deuten, dass es eine Zeit gegeben hat, wo man die Einschiebung als ein Konstruktionsmittel anerkannte, das unmittelbar bei geometrischen Konstruktionen neben Zirkel und Lineal angewandt werden durfte. Unter einer Einschiebung wird dann im allgemeinen die Konstruktion einer Strecke verstanden, deren Endpunkte auf gegebenen Linien liegen, und die selbst oder in ihrer Verlängerung durch einen gegebenen Punkt geht. Sie lässt sich einigermassen leicht mechanisch ausführen durch ein Lineal (oder ein gefaltetes Stück Papier), auf das man zwei Marken im Abstande der gegebenen Strecke abgetragen hat. Dieses Lineal dreht man um den festen Punkt, indem man es gleichzeitig so verschiebt, dass die eine Marke der einen gegebenen Linie folgt, und mit einer solchen Bewegung fährt man solange fort, bis die andere Marke sich auf der zweiten gegebenen Linie befindet.

Wegen des theoretischen Zieles, das die Griechen mit ihren Konstruktionen verfolgten, begnügten sie sich 
jedoch nicht lange mit dieser mechanischen Leichtigkeit. Da man überdies, um auf möglichst wenigen Voraussetzungen bauen zu könnnen, auch so wenige anerkannte Konstruktionsmittel haben musste wie nur möglich war, so wurde die unmittelbare Ausführung der Einschiebungen bald überall dort verdrängt, wo sie sich durch Zirkel und Lineal, die einzigen Konstruktionsmittel, die in Euklids Elementen Bürgerrecht erhalten, ausführen liessen. Möglicherweise sind ältere Anwendungen die Ursache, dass Apollonius zwei Bücher über Einschiebungen geschrieben hat, die, wie wir wissen, über die Ausführung dieser mit Hülfe von Zirkel und Lineal gehandelt haben. Er kann dadurch dem Mangel in älteren Werken haben abhelfen wollen, dass Aufgaben auf Einschiebungen zurückgeführt sind, ohne dass eine solche Ausführung angegeben wird.

Bei Einschiebungen, die sich nicht durch Zirkel und Lineal, sondern durch Benutzung von Kegelschnitten ausführen lassen, ist es auch von einem gewissen Zeitpunkt an obligatorisch geworden diese Kurven anzuwenden, sich also nicht mit der mechanischen Ausführung zu begnügen. Dass dies erst nach Archimedes geschehen sein sollte, kann man keineswegs mit voller Sicherheit daraus schliessen, dass er sich damit begnügt, Aufgaben auf Einschiebungen zurückzuführen; denn der Umstand, dass man sich früher mit einer mechanischen Ausführung begnügte, wird für ihre Ausführung durch Kegelschnitte feste Regeln hervorgerufen haben, die Archimedes als bekannt betrachten konnte. Wie die Einschiebungen des Archimedes sich durch Kegelschnitte ausführen lassen, ist später von Pappus angegeben worden.

Wo man die Einschiebungen nicht auf die Benutzung dieser anderen Konstruktionsmittel zurückgeführt hat, ja nicht hat zurückführen können, da ist eine theoretische Untersuchung der Einschiebung selbst erforderlich gewesen. 
Am besten hat dies geschehen können durch Aufstellung einer Definition und eine darauf gegründete Untersuchung derjenigen Kurve, die bei der oben beschriebenen mechanischen Konstruktion von dem einen Endpunkt der gegebenen Strecke durchlaufen wird, nämlich von dem, der nicht an die eine gegebene Linie gebunden ist. Durch die Schnittpunkte dieser Kurve mit der zweiten gegebenen Linie wird dann die Einschiebungsaufgabe gelöst. Eine solche Untersuchung ist auch, sogar nach Archimedes' Zeit, von Nikomedes in dem Falle vorgenommen worden, wo die erste der gegebenen Linien eine Gerade ist. Die erzeugte Kurve wird dann eine Konchoide genannt. Nikomedes hat zugleich einen Apparat erdacht um diese Kurve mechanisch zu erzeugen. Die Benutzung dieses Apparates deckt sich ungefähr mit der oben beschriebenen mechanischen Ausführung einer Einschiebung.

Wie nun auch die Einschiebung ausgeführt worden sein mag, so hat doch diejenige Zurückführung der Dreiteilung des Winkels, die wir - mit allem möglichen Vorbehalt - dem Archimedes beigelegt haben, eine grosse Bedeutung in der späteren Geschichte der Mathematik erhalten. Namentlich liegt sie der Lösung zu Grunde, die Vieta für Gleichungen 3ten Grades im sogenannten irreduciblen Fall gegeben hat.

\section{Verdoppelung des Würfels.}

Von den Aufgaben, die in ihrer algebraischen Form von Gleichungen 3ten Grades abhängig sind und später im Altertum durch Kegelschnitte gelöst wurden, war die Dreiteilung des Winkels nicht die einzige, die man bereits im 5ten Jahrhundert in Angriff genommen hatte. Von noch grösserer Bedeutung war die Aufgabe, die die geo- 
metrische Form der reinen kubischen Gleichung darstellt, nämlich die Verdoppelung oder Multiplikation des Würfels.

Diese Aufgabe heisst das delische Problem in Veranlassung eines Orakelspruches, wonach ein würfelförmiger Altar auf der Insel Delos doppelt so gross gemacht werden sollte ohne seine Form zu verändern; man darf indessen wohl annehmen, dass Pythia bei dieser Gelegenheit durch die Mathematiker inspiriert worden sei. Wie bereits erwähnt hatte man in der geometrischen Algebra Produkte von zwei allgemeinen Faktoren und Operationen mit den daraus zusammengesetzten Ausdrücken zweiten Grades umgeformt in Rechtecke und in Operationen mit Flächen, und in Verbindung damit das Ausziehen der Quadratwurzel vertauscht mit der Verwandlung eines Rechtecks in ein Quadrat, eine Aufgabe, die von den Pythagoreern gelöst worden sein soll. Da lag es denn nahe von diesen «ebenen» Aufgaben zu den entsprechenden «räumlichen» überzugehen. Man musste dann ein Produkt von 3 Grössen durch ein Parallelepipedon darstellen, und Operationen mit Ausdrücken vom 3ten Grade als Operationen mit Raumgebilden. Nächst so einfachen Dingen wie Einführung einer neuen Kante oder Grundfläche in ein Parallelepipedon und Anwendung davon auf Addition und Subtraktion, oder Verwandlung eines Parallelepipedons mit rechteckiger Grundfläche in ein solches mit quadratischer, musste die Aufgabe, ein Parallelepipedon in einen Kubus $\mathrm{zu}$ verwandeln, sich mit derselben Macht geltend machen, wie sich nach den Quadratwurzeln die Frage nach Kubikwurzeln demjenigen aufdrängt, der die Algebra in ihrer gegenwärtigen Gestalt aufbauen sieht. Wie $\sqrt[3]{2}$ die nächstliegende irrationale Kubikwurzel ist, wurde die Verdoppelung des Würfels das nächstliegende 
Beispiel für Aufgaben der hier bezeichneten Art. Als solche und durch die neuen Schwierigkeiten, die sie darbot, erweckte sie grosses Interesse bei den Mathematikern.

Der erste Beitrag zur Lösung dieser Aufgabe, den wir erwähnt finden, wird dem Hippokrates zugeschrieben. Ebenso wie die Verwaudlung eines Rechtecks in ein Quadrat auf der Konstruktion einer mittleren Proportionale beruht, soll er die Aufgabe von der Verdoppelung des Würfels, also vermutlich auch die etwas allgemeinere Aufgabe von der Verwandlung des Parallelepipedons in einen Kubus, auf die andere zurückgeführt haben, zwei mittlere Proportionalen $\mathrm{zu}$ bestimmen. Ist nämlich das Parallelepipedon bereits in ein solches $a^{2} b$ mit der quadratischen Grundfläche $a^{2}$ und der Höhe $b$ verwandelt, und soll dieses wieder in den Würfel $x^{3}$ verwandelt werden, so lässt sich $x$ bestimmen aus den Proportionen

$$
a: x=x: y=y: b \text {. }
$$

Ob nun diese Umformung dem Hippokrates zuzuschreiben ist oder nicht, nach ihm erscheint das Delische Problem gewöhnlich unter der Form der Aufgabe: zwei mittlere Proportionalen $x$ und $y$ zu bestimmen zu den gegebenen Strecken $a$ und $b$.

Die erste von den vielen Lösungen, die diese Aufgabe im Altertum erfahren hat, verdankt man dem Ar-

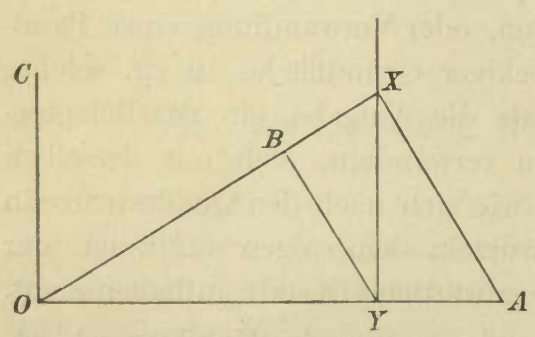
chytas. Um diese Lösung recht $\mathrm{zu}$ verstehen, muss man festhalten, dass er darauf ausgeht eine Figur zu konstruieren, die aus zwei Geraden $O Y A$ und $O B X$ besteht, zwischen denen die gebrochene Linie $A X Y B$ so gezeichnet werden soll, dass $X Y$ senkrecht auf der ersten, $A X$ und 
$Y B$ senkrecht auf der zweiten stehen, während $O A$ und $O B$ von gegebener Länge sind. Dann sind nämlich offenbar $O X$ und $O Y$ die beiden mittleren Proportionalen zwischen $O A$ und $O B$. Man kennt also den Durchmesser $O A$ eines Kreises, auf dem $X$ liegen soll, aber nicht den Durchmesser $O Y$ eines Kreises, auf $\operatorname{dem} B$ liegen soll. Archytas sucht diesen letzten Kreis einzuführen als Schnittkreis der Kugel über $O A$ als Durchmesser. Da $O B$ gegeben ist, so wird der Punkt $B$ auf einem Schnittkreis dieser Kugelfläche liegen, die Linie $O B$ und dadurch der Punkt $X$ auf dem Umdrehungskegel, der diesen bekannten Schnittkreis zur Leitlinie hat. Wenn man nun versucht die verlangte Stellung zu erreichen durch eine Drehung der Figur um die in ihrer Ebene in $O$ auf $O A$ errichtete Senkrechte $O C$, so wird die Projektion $Y$ des Punktes $X$ auf die von $O A$ durchlaufene Ebene einen grössten Kreis beschreiben, die Linie $X Y$ also eine Cylinderfläche, auf der der Punkt $X$ auch liegen muss.

Da nun $X$ ferner während der Umdrehung dauernd auf dem Kreise über $O A$ als Durchmesser liegen soll, so muss er auf der Kurve liegen, die dieser Kreis während seiner Bewegung auf der Cylinderfläche aufzeichnet, d. h. in Wirklichkeit auf der Schnittlinie der Cylinderfläche mit dem durch die Umdrehung des Kreises um seine Tangente in $O$ erzeugten Wulst. Der Punkt $X$ wird dann bestimmt durch den Durchschnitt zwischen dieser cylindrischen Raumkurve und der obengenannten Kegelfläche, und durch seine Bestimmung ist die Aufgabe gelöst.

Diese Lösung wird kaum zu einer wirklich durchgeführten praktischen Bestimmung angewandt worden sein. Hierauf deutet unter anderem der Umstand, dass die wirkliche Erzeugung der Raumkurve nicht genannt wird, denn der hierzu dienende Wulst ist nur eine von uns 
eingeschobene Erklärung. Archytas hat sicher erkennen können, dass man durch successives Probieren leichtere und genauere Bestimmungen von $A X$ und $A Y$ erhält. Das was hier beabsichtigt war, war also eine theoretische Bestimmung, die bei weitergehenden Untersuchungen, bei denen Kubikwurzeln vorkommen, benutzt werden konnte. Damit diese in dieser Beziehung wirklich befriedigend hätte sein können, müsste man jedoch dem Archytas eine Bekanntschaft mit der benutzten Raumkurve oder doch mit Hülfsmitteln um ihre Eigenschaften anzugeben beilegen, die er schwerlich besessen haben wird.

Seine Lösung erhält dagegen grossen Wert für uns als ein unmittelbares Zeugnis für das, was er zu leisten vermochte. $\mathrm{Er}$ ist auf seine Aufgabe losgegangen mit dem Gedanken an die Anwendung des Kreises zur Lösung der entsprechenden ebenen Aufgabe. Er versucht, ob die Kugel sich nicht auf entsprechende Weise für die Lösung der vorliegenden räumlichen Aufgabe sollte verwenden lassen, und er führt diesen Versuch durch mit klarer Erfassung der räumlichen Verhältnisse, die sich dabei darbieten, ja er schreckt nicht zurück vor der Einführung einer Kurve, die ein gewisser Kreis während seiner Bewegung auf einem Cylinder aufzeichnet. Ausser von einem sicheren Gedankengang bei ihm selbst zeugt seine Konstruktion von einem Vertrautsein mit der Anwendung geometrischer Örter zur Bestimmung von Punkten, die hinreichend entwickelt war, um ihre Erweiterung auf den Raum vornehmen zu können. Wir dürfen daraus schliessen, dass die Geometrie des Raumes und die Anwendung geometrischer Örter wenigstens in der Ebene zu seiner Zeit bereits zu einer recht bedeutenden Entwickelung gelangt war.

Es wird berichtet, dass Archytas' Schüler Eudoxus zur Lösung derselben Aufgabe einige andere Kurven be- 
nutzt habe. Man hat geraten auf Projektionen der Schnittkurven zwischen den 3 Flächen, die in Wirklichkeit bei der Konstruktion des Archytas benutzt werden. Eudoxus' Schüler Menächmus verfiel dagegen darauf das Hülfsmittel zu benutzen, das später im griechischen Altertum auf diese und viele andere Aufgaben angewandt wurde, nämlich die Kegelschnitte. Nach den Berichten späterer Schriftsteller soll er die beiden mittleren Proportionalen zwischen $a$ und $b$ bestimmt haben als die Koordinaten $x$ und $y$ der Schnittpunkte zwischen den durch zwei von den Gleichungen

$$
a y=x^{2}, b x=y^{2}, x y=a b
$$

bestimmten Kurven, und zugleich soll er gezeigt haben, wie diese Kurven, die ja Parabeln und eine Hyperbel werden, sich stereometrisch als Schnitte an Umdrehungskegeln darstellen lassen. $\mathrm{Zu}$ diesen Bestimmungen werden wir zurückkehren, sobald wir, nachdem wir in unserer allgemeinen Untersuchung weiter fortgeschritten sein werden, die Entwickelung der Lehre von den Kegelschnitten im Zusammenhange behandeln.

Hier ist dagegen der Ort um noch solche Anwendungen anderer Hülfsmittel zu berühren, die man noch weithin in der folgenden Zeit fortfuhr für die Konstruktion der beiden mittleren Proportionalen ausfindig zu machen. Man erfand verschiedene mechanische Werkzeuge für die Konstruktion einer Figur, die wie die Figur auf S. 84 ähnliche Dreiecke enthalten, durch die sich unmittelbar die verlangte Verbindung ergiebt. Eines von diesen wird Plato zugeschrieben, ein zweites rührt von Eratosthenes her. Da sich indessen ergiebt, dass keiner von diesen Apparaten eine wirkliche Bedeutung für die Entwickelung der Mathematik gehabt hat, so wollen wir uns eine Beschreibung von ihnen und ihrem Gebrauch ersparen und uns damit begnügen $\mathrm{zu}$ bemerken, dass 
diese Apparate Descartes veranlasst haben gleichfalls einen zu erdenken, den er in seiner Geometrie beschreibt.

Die Konstruktion der beiden mittleren Proportionalen ist auch von Nikomedes auf eine Einschiebung zurückgeführt worden. Die hierzu dienende Konstruktion ist jedoch keineswegs so einfach wie diejenigen sind, die für die Dreiteilung des Winkels benutzt werden.

\section{Theoreme und Probleme; Bedeutung der geometrischen Konstruktion.}

Wir haben teils über die Hauptanschauungen und die sich daran schliessenden Operationsmethoden gesprochen, die im 5ten Jahrhundert ihren Anfang nahmen und sich in der folgenden griechischen Mathematik weiter entwickelten, teils durch Erwähnung einzelner Untersuchungen Proben von dem damaligen reellen Inhalt dieser Mathematik gegeben. In dem Maasse wie man fortschritt bedurfte man fester und zuverlässiger Formen, die zu diesen Anschauungen stimmten und sie dadurch in noch höherem Grade sicher stellten, und die dem stets wachsenden Inhalt in sich Raum gewährten. Die hierzu führende Arbeit wurde in Platos philosophischer und $\mathrm{Eu}$ doxus' mathematischer Schule und durch Verhandlungen zwischen beiden ausgeführt.

Als Beispiel für eine solche Verhandlung können wir einen Streit darüber anführen, in wie weit die mathematischen Wahrheiten als Theoreme (Lehrsätze) oder als Probleme (Aufgaben) auftreten dürfen. Das erste wurde von den Platonikern geltend gemacht, die sich darauf stützten, dass die Lösung einer Aufgabe nur etwas zustande bringe, was schon im voraus vorhanden sei: gleichseitige Dreiecke existieren unabhängig davon, ob 
man sie konstruiert, und man kann ein solches nur deshalb konstruieren, weil der Begriff «gleichseitiges Dreieck» eine Realität hat, bevor man es konstruiert. Für die Schüler des Eudoxus, die bei dieser Gelegenheit namentlich von Menächmus repräsentiert wurden, war die mathematische Hervorbringung durch Konstruktion oder doch durch Untersuchung der Figur die Hauptsache.

In äusserer Hinsicht scheint keine der Parteien die andere besiegt zu haben, da Theoreme und Probleme neben einander in Euklids Elementen vorkommen. Von grösserer Bedeutung ist die Prüfung dessen gewesen, was ausser der rein äusseren Form Theoreme und Probleme charakterisiert. Das hat man wenigstens später etwa folgendermassen ausgedrückt: im Theoreme wird das einzig mögliche ausgesagt, im Probleme wird das verlangt, was anders sein könnte. Nach diesen Kennzeichen muss man entscheiden, ob eine Wahrheit in der einen oder anderen Form mitgeteilt werden soll. Beispielsweise würde es unrichtig sein als Problem zu stellen: "Einen rechten Peripheriewinkel zu konstruieren, der auf einem Halbkreise steht.»

Wichtiger als solche Bestimmungen in Worten ist es jedoch die Rolle kennen zu lernen, die Theoreme und namentlich Probleme bei den uns erhaltenen Schriftstellern spielen, namentlich in Euklids Elementen. Vielleicht begreift man dadurch auch besser die von Menächmus verfochtene Ansicht als durch die überlieferte Mitteilung. Diese lässt die Platoniker geltend machen, dass das gleichseitige Dreieck existiert, bevor es konstruiert wird. Im Gegensatz hierzu kann Menächmus behauptet haben, dass man erst erfährt, dass es wirklich existiert, wenn man es konstruiert und damit den Beweis verbindet, dass diese Konstruktion wirklich zum Ziele führt. So verfährt Euklid, indem er sich nicht damit begnügt gleichseitige 
Dreiecke zu definieren, sondern, bevor er weiteren Gebrauch von ihnen macht, sich ihrer Existenz dadurch versichert, dass er im ersten Satze des ersten Buches die Aufgabe löst ein solches zu konstruieren und die Richtigkeit der Konstruktion beweist.

Die Notwendigkeit eines solchen Verfahrens macht sich insofern von selbst geltend, als gleichseitige Dreiecke demnächst bei neuen Konstruktionen benutzt werden sollen; aber es ist beachtenswert, dass Euklid auf dieselbe Weise mit solchen Dingen verfährt, die in der Folge nur im Beweise für einen Lehrsatz benutzt werden sollen. Bevor er in I, 16 die Mitte einer geradlinigen Strecke benutzen darf, muss er in I, 10 durch Konstruktion dieses Punktes bewiesen haben, dass er wirklich existiert. Etwas ähnliches gilt für alle ähnlichen Fälle. Die wesentliche Bedeutung der geometrischen Konstruktion liegt darin, dass sie zum Beweise dafür dienen soll, dass dasjenige, auf dessen Darstellung die Konstruktion ausgeht, wirklich existiert.

Mag nun auch Menächmus zuerst diese Bedeutung der geometrischen Probleme, die durch Konstruktion gelöst werden, $\mathrm{zu}$ vollem Bewusstsein gebracht haben, so hat diese sich doch auch schon früher geltend gemacht. Das hängt nämlich auf das genaueste zusammen mit der geometrischen Algebra. Als man gefunden hatte, dass keine Zahl oder kein Zahlenverhältnis (Bruch) existiert, die mit sich selbst multipliciert 2 ergeben, und als man, statt eine solche Zahl zu verlangen, eine Strecke verlangte, welche die Seite eines Quadrates ist von doppelter Grösse wie das Quadrat über einer gegebenen Strecke, so musste man die Existenz einer solchen Strecke beweisen. Das geschieht dadurch, dass man sie als Diagonale des Quadrates über der gegebenen Strecke darstellt. Eine ähnliche Bedeutung erhält die Lösung allgemeiner Glei- 
chungen 2ten Grades durch eine Konstruktion. Erst mit dieser allgemeinen Auffassung vor Augen begreift man vollkommen den Wunsch nach einer konstruktiven Lösung von der Quadratur des Kreises, der Dreiteilung des Winkels, der Verdoppelung des Würfels und der Bestimmung der beiden mittleren Proportionalen. Ohne sie kann man nämlich durchaus nicht begreifen, dass die zum technischen Gebrauch ungeeigneten Lösungen, wie die von der Quadratur des Kreises durch die Quadratrix und wie die Bestimmung der mittleren Proportionalen durch Archytas, überhaupt irgendwelche Befriedigung gewähren konnten. Dieselbe Auffassung wird auch den Schlüssel zum Verständnisse anderer Verhältnisse in der griechischen Mathematik abgeben.

In gewissen Fällen wird übrigens diese Benutzung der Konstruktionen auch uns nicht fern liegen. Das gilt namentlich, wenn eine ganz allgemein gestellte Aufgabe nicht immer möglich ist, sondern gewisse Bedingungen für ihre Möglichkeit verlangt. In solchen Fällen beginnen die griechischen Schriftsteller damit, die Notwendigkeit dieser Bedingungen nachzuweisen. Das geschieht durch den Beweis für ein Theorem, das ausspricht, dass die betreffende Figur immer die Eigenschaften besitzt, die die Bedingungen für die Möglichkeit verlangen. Dass diese Bedingungen ausreichend sind, wird demnächst in einem Probleme bewiesen, indem angegeben wird, wie die Figur $\mathrm{zu}$ konstruieren ist, wenn sie erfüllt sind, und bewiesen wird, dass die Figur dann wirklich zustande gebracht ist. Das erste Beispiel hierfür besitzen wir in Euklid I, 20 und 22. Der erste Satz enthält den Lehrsatz, dass jede Seite eines Dreiecks kleiner ist als die Summe der beiden anderen, der zweite das Problem ein Dreieck zu konstruieren, dessen Seiten gegeben sind, wenn alle drei dieser Bedingung genügen. 


\section{Die analytische Methode; die analytisch-synthetische Darstellungsform.}

Der wichtigste Beitrag, den die Schulen von Plato und Eudoxus geliefert haben um der Mathematik die äussere Form zu geben, in der sie bei Euklid und den folgenden griechischen Mathematikern erscheint, ist gewiss die Ausgestaltung der sogenannten apagogischen oder analytischen Methode und der Formen Analyse und Synthese, durch die man sich sowohl zuverlässige Frgebnisse ihrer Anwendung als auch eine unanfechtbare Darstellung dieser Ergebnisse sicherte.

Die analytische Methode findet zu allernächst Anwendung bei der Lösung von Aufgaben, und deshalb wollen wir zuerst von ihr reden. Wir glauben indessen, dass die logische Bedeutung der Regeln, die aufgestellt wurden um die Lösung zu finden und darzustellen, sich am besten verstehen lässt, wenı wir für einen Augenblick das Gebiet der griechischen Mathematik verlassen und von der analytischen Lösung von Aufgaben ganz im allgemeinen sprechen und zum Teil ihre Anwendung durch Beispiele klar machen, die anderen Aufgaben und anderen Hülfsmitteln, als den Griechen zu Gebote standen, entnommen sind. Ich beabsichtige dadurch auf einen $\mathrm{zu}$ dem ursprünglichen stimmenden konsequenten Gebrauch hinzuweisen, der sich in der Mathematik von den Worten Analyse und Synthese, analytisch und synthetisch machen lässt, und der Platz greifen müsste statt der Verwirrung, zu der in der neueren Zeit die ausschliessliche Anwendung des Wortes Analyse auf die algebraische Analysis den ersten Anstoss gegeben hat.

Eine mathematische Aufgabe geht darauf aus Grössen oder Figuren zu finden, die gewissen Forderungen genügen. 
Bei ihrer Lösung kann ein Erraten, dass sich auf Ähnlichkeiten mit anderen Aufgaben gründet, oft wohl eine Rolle spielen, und es soll nicht geleugnet werden, dass wichtige mathematische Resultate zuerst auf diesem Wege erreicht sein können; aber ein solches Erraten liegt ausserhalb jeder eigentlichen Methode. Bei jeder methodischen Behandlung wird es darauf ankommen, die gestellten Forderungen $\mathrm{zu}$ "analysieren». Man muss sie zuerst klar in Gedanken festhalten, was nur dadurch geschehen kann, dass man sie sich erfüllt, die Aufgabe also gelöst denkt. Demnächst kommt es darauf an, auf irgend eine Weise, nach Regeln, die von derartigen Aufgaben her bekannt sind, oder nach neu gefundenen Regeln, die Forderungen in neue umzuformen, die notwendigerweise erfüllt sind, wenn die ersten es sind, und diese Umformung fortzusetzen, bis man zuletzt zu Forderungen gelangt, die man zu erfüllen imstande ist.

Bei dieser Analyse findet man, wie die Aufgabe gelöst werden muss, wenn sie sich überhaupt lösen lässt. Die Synthese besteht dann zuerst in der wirklichen Ausführung dieser Lösung: in einer solchen Bestimmung der gesuchten Grössen und Figuren, dass die umgeformten Forderungen befriedigt sind. Danach wird noch ein $\mathrm{Be}-$ weis dafür verlangt, dass dann auch die ursprünglich gestellten Forderungen befriedigt sind. Dieser Beweis lässt sich, wenn sich keine einfacheren Wege darbieten, in der Regel führen durch eine Umformung der Forderungen in der entgegengesetzen Reihenfolge wie diejenige war, die bei der Analyse benutzt wurde, so dass man damit schliesst, dass die Erfüllung der neuen Forderungen, die man an die Stelle der ursprünglichen gesetzt hat, auch notwendigerweise die Erfüllung dieser mit sich führt. Der Beweis kann fortgelassen werden oder ist bereits in der Analyse geführt, wenn man in dieser 
nur solche Umformungen benutzt hat, die sich umkehren lassen, so dass die neuen Forderungen nicht nur die notwendigen, sondern auch die ausreichenden Bedingungen für die alten sind, aber sonst nicht.

Als Beispiel wollen wir die Lösung von Aufgaben durch algebraische Gleichungen nehmen. Indem man Benennungen für die unbekannten Grössen einführt und diese auf ganz dieselbe Weise wie die Bezeichnungen für bekannte Grössen in die Gleichungen eintreten lässt, die die gegebenen Forderungen ausdrücken, denkt man sich diese Gleichungen befriedigt, also die Aufgabe gelöst. Die vorhin erwähnte Umformung der Forderungen wird dargestellt durch die Umformung der Gleichungen, bis man zu solchen Gleichungen gelangt, die die Lösung ergeben. Das kann z. B. in der analytischen Geometrie ${ }^{1}$ geschehen durch Herstellung der Gleichungen für solche geometrischen Örter, durch welche die Aufgabe gelöst wird. Wird die Analyse auf Aufgaben angewendet, die darauf ausgehen die Werte von Unbekannten zu finden, so werden die umgeformten Gleichungen diejenigen sein, in denen die Unbekannten isoliert sind. Wenn wir uns nur an diesen letzten Fall halten, so besteht die auf die Analyse folgende Synthese 1) in der wirklichen Ausrechnung der durch die gefundenen Ausdrücke gegebenen Grössen, ihre Umformung nach bestimmten Regeln, z. B. ihre Verkürzung, Reduktion auf einfache Irrationalität etc. mit einbegriffen, 2) in einer Prüfung dieser Grössen.

${ }^{1}$ Die Bezeichnung «analytische Geometrie» wollen wir in der gewöhnlichen Bedeutung benutzen, ohne Rücksicht darauf, dass auch andere Geometrie analytisch sein kann, und dass man auch synthetisch mit den Hülfsmitteln operieren kann, die nun einmal den Anspruch erworben haben analytische Geometrie genannt zu werden. 
Diese wird wohl in der Regel durch direktes Einsetzen vorgenommen, lässt sich aber auch in Übereinstimmung mit dem, was oben über die Bildung des synthetischen Beweises gesagt wurde, dadurch ausführen, dass man Schritt für Schritt durch die benutzten Gleichungen in umgekehrter Ordnung zurückgeht. Dass ein solcher Beweis nicht an und für sich durch die vorangehende Analyse überflüssig gemacht wird, weiss man aus solchen Fällen, in denen man durch Potenzieren einen Wurzelausdruck fortgeschafft hat. War dieser einer von den Werten der Wurzel, z. B. der positive Wert einer Quadratwurzel, so weiss man, dass man dann fremde Lösungen einführen kann. Statt eines Beweises für die Richtigkeit liefert die Probe dann einen Beweis für die Unrichtigkeit dieser letzten Wurzeln. Die Analyse allein lässt also die Möglichkeit für fremde Lösungen offen. Ist die Lösung im voraus bekannt, so kann sie und ihr Beweis allein synthetisch mitgeteilt werden, aber damit ist ein anderer Übelstand verbunden. Ist die Aufgabe beispielsweise die Gleichung

$$
x^{2}-a x+b=0
$$

oder eine Aufgabe, die, auf eine Gleichung gebracht, so ausgedrückt werden würde, so kann man synthetisch mitteilen, dass

$$
x=\frac{a}{2}+\sqrt{\left(\frac{a}{2}\right)^{2}-b},
$$

und unter dem Quadratwurzelzeichen die positive Quadratwurzel verstehen. Die Probe oder der synthetische Beweis ergiebt, dass diese Lösung richtig ist; man sieht aber nicht, ob sie die einzige richtige ist.

Was hier von der algebraischen Lösung nachgewiesen ist, gilt allgemein: Die Analyse allein kann $\mathrm{zu}$ viele Lösungen geben, die Synthese allein zu wenige. 
Noch wollen wir untersuchen, auf welchem Punkte der vollständigen Behandlung sich die Bedingungen für die Möglichkeit darbieten. Man pflegt sie heut zu Tage an die vollständige Beendigung der formellen Auflösung anzuschliessen, die man dann diskutiert. In dem ebengenannten Beispiele wird aus dem gefundenen Ausdruck geschlossen, dass, damit $x$ reell sein kann, $\left(\frac{a}{2}\right)^{2} \geq b$ sein muss. Eine solche Diskussion wird jedoch nur dadurch möglich, dass man durch Einführung der Benennungen «negative und imaginäre Grössen» auch solche Grössen anerkennt, die man ursprünglich nicht als Lösungen erwartet hatte. Ohne diese neuen Arten von Grössen würde man schon an einem früheren Punkte der Analyse auf die Bedingung für die Möglichkeit getroffen sein. Wenn man beispielsweise aus der obenstehenden Gleichung abgeleitet hat, dass

$$
\left(x-\frac{a}{2}\right)^{2}+b=\left(\frac{a}{2}\right)^{2},
$$

so kann man daraus nur

$$
\left(x-\frac{a}{2}\right)^{2}=\left(\frac{a}{2}\right)^{2}-b
$$

ableiten, wenn $\left(\frac{a}{2}\right)^{2} \geqq b$, da die rechte Seite sonst keinen Sinn giebt. Die Bedingungen für die Möglichkeit werden also aus der Analyse ebenso abgeleitet wie die Aufösung, lassen sich aber ebenso wie diese in einer rein synthetischen Darstellung mitteilen.

Das Gebiet, auf welches die Griechen die hier geschilderte Methode zur Lösung von Aufgaben anwandten, sind die geometrischen Aufgaben, deren Endziel, wie wir gesehen haben, im allgemeinen eine Konstruktion ist, entweder eine wirkliche mit Hülfe von Zirkel und 
Lineal, oder eine formelle. Solange man methodisch die Lösung solcher Aufgaben gesucht hat, muss man nach unseren allgemeinen Bemerkungen analytisch zu Werke gegangen sein. Eine solche Behandlungsweise muss gewiss bereits gebraucht worden sein bei der geometrischen Lösung der Gleichungen zweiten Grades durch die Pythagoreer. Die Methode kann indes mehr oder minder bewusst angewandt worden sein, denn, wie es sich oft in der Geschichte der Mathematik gezeigt hat, ist faktisch eine Methode $\mathrm{zu}$ benutzen nicht dasselbe wie sie sich so klar zu machen, dass man sie jedesmal zur Verfügung hat, wenn sich der Bedarf danach herausstellt, geschweige denn sie so aufzustellen, dass sie auch anderen zur Verfügung steht.

Eine Konstruktion, die in ausgeprägter Weise zeigt, dass sie durch Anwendung der analytischen Methode gefunden worden ist, ist Archytas' Bestimmung vọn zwei mittleren Proportionalen. Er konnte nämlich unmöglich die Anwendung der ihm im voraus unbekannten cylindrischen Kurve erraten haben, und deshalb muss es ausschliesslich die von ihm angewandte Analyse gewesen sein, die ihn zu ihrer Einführung gezwungen hat, ganz wie wenn in einer modernen analytisch-geometrischen Untersuchung ein geometrischer Ort, der bei der Lösung der Aufgabe zur Verwendung kommt, sich als eine Kurve herausstellt, von der man vorher nichts wusste, die aber durch die aus der Analyse hervorgehende Gleichung definiert wird. Während wir hierdurch eine, bereits benutzte, Andeutung darüber erhalten, dass die Zurückführung der Aufgaben auf die Benutzung geometrischer Örter und die Bestimmung dieser Örter zu den Seiten der analytischen Methode gehörte, die bereits eine gewisse Entwickelung bei den Pythagoreern gefunden hatten, erfuhr die Methode durch die von Archytas' Nachfolgern Plato 
und Eudoxus gestifteten Schulen die formelle Entwickelung, die danach bei den griechischen Mathematikern in dauerndem Gebrauche blieb.

Die Anwendung der Methode und die Darstellung der durch sie gewonnenen Resultate bestand in einer Reihe von Gliedern, deren Beschreibung wir am leichtesten an die elliptische Flächenanlegung (S. 47) als Beispiel anknüpfen können. Indessen wollen wir die einzelnen Glieder etwas kürzer ausdrücken, als die Alten es gethan haben würden.

1) Die Aufgabe wird gestellt in der sogenannten

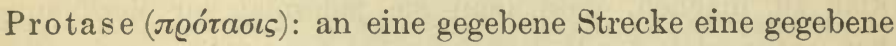
Fläche (Quadrat) so anzulegen, dass ein Quadrat fehlt.

2) Die Aufgabe wird ausgesprochen mit Bezug auf eine bestimmte, gezeichnete Figur in der Ekthese ('̌ $\% \vartheta \varepsilon \sigma \varsigma)$ :

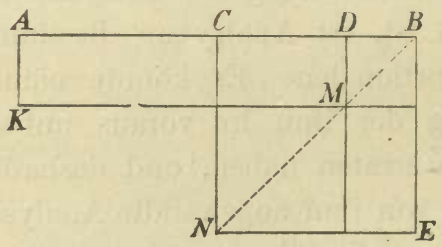

das (gezeichnete) Quadrat $q$ soll als Rechteck an die Strecke $A B$ so angelegt werden, dass ein Quadrat fehlt.

3) Man denkt sich die Aufgabe gelöst (durch das Rechteck $A M$, das das Quadrat $B M$ fehlen lässt) und führt sie in der Apagoge, der Transformation (å $\left.\tau \alpha \gamma \omega \gamma \eta^{\prime}\right)$, zurück auf eine bekannte Aufgabe: Ist $C$ die Mitte von $A B$, so wird das Rechteck $K C$ hin auf $D B$ gelegt (als $D E$ ). Dadurch wird das Rechteck $A M$ verwandelt in einen Gnomon oder in die Differenz zwischen den Quadraten $C B^{2}$ und $C D^{2}$. Die Strecke $C D$ muss also 
so bestimmt werden, dass dieser Gnomon dem Quadrate $q$ gleich wird.

4) In der Resolution, wie man sie genannt hat, wird demnächst auseinandergesetzt, wie weit man nun wirklich alles besitzt, was notwendig ist um die gestellte Aufgabe zu lösen. Im vorliegenden Falle findet das nur statt, wenn $q$, das eben so gross sein soll wie ein von dem Quadrat $C B^{2}$ abgeschnittener Gnomon, kleiner als dieses Quadrat ist. Wenn man dies bemerkt hat, so hat sich dadurch allerdings ein Mangel an der gestellten Aufgabe herausgestellt. Es ist nämlich, wenigstens in der überlieferten Litteratur, Regel, dass die Aufgaben mit einer solchen Begrenzung gestellt werden, dass sie gelöst werden können. Dadurch erhält man statt der Aufgabe, die wir hier $\mathrm{zu}$ stellen versucht haben, teils ein Theorem, teils ein enger begrenztes Problem. Das Theorem (das sich in einer etwas allgemeineren Form in Euklid VI, 27 findet) muss darauf hinaus laufen, dass ein Rechteck, so an eine Strecke angelegt, dass ein Quadrat fehlt, kleiner ist als das Quadrat über der halben Strecke, oder, wenn man will, dass ein Rechteck kleiner ist als ein Quadrat von demselben Perimeter. Das Problem (das in allgemeinerer Form in Euklid VI, 28 behandelt wird) wird dasselbe wie dasjenige, dessen Lösung hier versucht ist, nur mit dem Zusatz, dass das gegebene Quadrat kleiner sein muss als das Quadrat über der Hälfte der gegebenen Strecke. Dieser Zusatz zur Protase wird der Diorismus

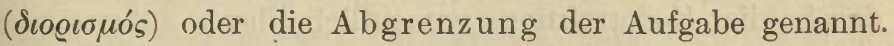
In der Ekthese muss ferner von den Figuren, die man annimmt, ausgesprochen werden, dass sie die Bedingung $\left(q<C B^{2}\right)$ erfüllen. Durch die Abgrenzung, die wir uns nun also in die Protase und Ekthese eingeführt denken, wird, wie wir sehen werden, die Resolution ganz überflüssig; denn nun wird der Versuch, ob die Aufgabe sich 
durch das Vorliegende lösen lässt, gelingen, und die Resolution wird dann mit der in der Synthese folgenden Angabe, wie sie zu lösen ist, ganz zusammenfallen. Sehen wir dagegen nicht auf die überlieferten Mitteilungen über Resultate von zh Ende geführten Untersuchungen, sondern auf die Anwendung der Methode auf neue Untersuchungen, so muss die Resolution eine wichtige Rolle gespielt haben. Während der Analyse hat man nämlich beständig prüfen müssen, ob die Apagoge weit genug geführt sei um die Aufgabe lösen zu können; ausserdem ist aber die Resolution ein Mittel gewesen um das zu erreichen, was wir bereits (S. 92) als ein Hauptziel für die Behandlung von Aufgaben genannt haben, nämlich die ursprüngliche Aufgabe in das Theorem und in das Problem zu zerlegen, durch die man sich versichert, dass die Bedingungen für die Existenz der verlangten Figur beziehungsweise notwendig und ausreichend sind. Sowohl in dem angeführten Beispiel wie im allgemeinen ist das, was man durch diese Methode erreicht hat, die Bestimmung eines Maximums oder eines Minimums.

Was die Resolution auch liefern sollte, das ist die Zahl der Auflösungen. So ist im vorliegenden Falle zu bemerken, dass es, wenn $C D^{2}$ die richtige Grösse erhält, gleichgültig ist, ob $D$ auf die eine oder die andere Seite von $C$ fällt, ein Umstand, auf den man gleichzeitig mit der Entdeckung des Maximalwertes von $q$ hat aufmerksam werden können. Indessen legten die Griechen, die sich durch die Konstruktion namentlich davon überzeugen wollten, dass die Figur überhaupt existierte, darauf kein sonderliches Gewicht. Da in anderen Fällen die Mehrdeutigkeit einer Aufgabe auf der Mehrdeutigkeit derjenigen beruht, auf die sie zurückgeführt wird, so wird sie, wenn sie bei den letzteren unbeachtet geblieben ist, auch nicht bei der Analyse der ersteren bemerkt worden sein. Wegen 
dieser Unterlassung mussten Fälle, bei denen die Mehrdeutigkeit den Griechen von einiger Wichtigkeit $\mathrm{zu}$ sein schien, zum Gegenstande besonderer Untersuchung gemacht werden.

Die Transformation und Resolution machen die Analyse aus, durch welche die Auflösung gefunden wird. Darauf wird die gefundene Lösung in der Synthese dargestellt. Diese enthält:

5) Die Konstruktion ( $\alpha \tau \alpha \sigma \varkappa \varepsilon v \eta ́)$, in der das Gesuchte mit Hülfe der anerkannten Konstruktionsmittel zustande gebracht wird. Es ist jedoch keine Rede davon alle Einzelheiten namhaft zu machen, sondern nur davon, die von früher her bekannten Konstruktionen anzugeben, aus denen die verlangte sich zusammensetzen lässt: in unserem Beispiel die Bestimmung von $C D$ durch den pythagoreischen Lehrsatz u. s. w. Die Konstruktion wird also nur mit einer kleinen Formveränderung eine Wiederholung dessen, was in der Resolution gesagt ist.

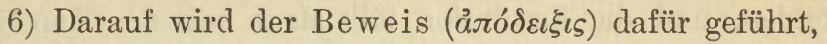
dass die Konstruktion wirklich die verlangte Figur zustande gebracht hat. Dieser wird in der Regel durch Anwendung derselben Schlüsse geführt, die in der Transformation in umgekehrter Reihenfolge benutzt worden sind. So wird im Beispiel das Rechteck $A M$ aus der Gnomonfigur dadurch gebildet, dass das Rechteck $D E$ auf $A C$ gelegt wird.

7) Endlich legt man sich in der Konklusion ( $\sigma v \mu$ $\pi \varepsilon ́ \varrho \alpha \sigma \mu \alpha)$ Rechenschaft darüber ab, dass man wirklich das verlangte Ziel erreicht hat. Das geschieht durch eine Wiederholung der Protase, eingeleitet durch «also ist u.s.w.» und abgeschlossen durch "was zu thun war».

Während die Analyse, die in 3 und 4, der Transformation und Resolution, enthalten ist, namentlich methodische Bedeutung für das Finden der Auflösung gehabt 
hat, ist sie nicht notwendig, wenn es nur darauf ankommt das Gefundene auf eine unanfechtbare Weise darzustellen, und das war stets der Hauptzweck bei den schriftlichen Mitteilungen der Griechen. Sie wird deshalb sehr oft ausgelassen, so dass die Darstellung nur noch aus den Abschnitten besteht, die wir hier mit 1, 2, 5, 6, 7 bezeichnet haben; dadurch gelangt man $\mathrm{zu}$ einer Darstellungsform, die wir synthetisch nennen würden. Diese synthetische Darstellungsform wird namentlich benutzt bei der systematischen Behandlung einer ganzen Theorie, deren einzelne Konstruktionen den Verfassern im voraus mehr oder weniger bekannt gewesen oder in weiterem $\mathrm{Zu}$ sammenhange gefunden sind, wie in Euklids Elementen und in dem grössten Teile von Apollonius' Lehre von den Kegelschnitten. Übrigens erfährt man eigentlich auch nicht mehr an den Stellen, wo die Analyse mitgeteilt wird; denn erstens lässt sich nach dem Gesagten die Transformation durch Umkehrung aller Schlüsse des Beweises bilden, und die Resolution fällt dann mit der Konstruktion zusammen; und zweitens ist die Analyse, die mitgeteilt wird, nur die Analyse der durch den Diorismus abgegrenzten Aufgabe und nicht - wie in unserem ursprünglichen Beispiele - diejenige, die zur Abgrenzung geführt hat.

Nachdem wir so ausführlich über die Analyse und die damit verbundene synthetische Darstellung von Problemen gesprochen haben, können wir rascher über die Anwendung dieser Methode und der entsprechenden Formen auf Theoreme hinweggehen. Die synthetische Darstellungsform besteht hier zunächst aus ganz denselben Gliedern oder kann jedenfalls daraus bestehen; nur muss man in diesen ïberall Theorem an die Stelle von Problem setzen. Die Konstruktion besteht hier nur in der Konstruktion der zum Beweise erforderlichen Hülfslinien, und 
fehlt, wenn solche nicht nötig sind, und die Konklusion schliesst hier mit den Worten «was zu beweisen war». Diese selben Glieder, die, wie man sieht, auch für die Theoreme logisch ausreichend sind, findet man deshalb, sowohl was Probleme als was Theoreme betrifft, überall bei Euklid.

Indessen kann auch mit Bezug auf Theoreme von einer eigentlich analytischen Methode die Rede sein. Diese lässt sich benutzen, wenn man prüfen will, ob ein Theorem, das von anderen mitgeteilt ist, oder dessen Aufstellung vielleicht durch Erraten geschehen ist, richtig ist oder nicht. Man beginnt damit, die Richtigkeit des Theoremes, das wir $A$ nennen wollen, vorauszusetzen; dann formt man dieses, ganz wie in der bei den Problemen angewandten Apagoge oder Transformation durch eine Reihe von Schlüssen um, bis man sieht, dass es zu einem neuen Resultate $K$ führt, von dem man weiss, dass es richtig ist oder falsch. Im ersten Falle ist noch nur eine Möglichkeit dafür vorhanden, dass $A$ richtig ist, aber keinerlei Gewissheit. $K$ kann aus einer Schlussreihe hervorgegangen sein, in der nur scheinbar Gebrauch von $A$ gemacht ist; auch wenn man moderne algebraische Hülfs. mittel benutzt, kann das geschehen, z. B. wenn man auf beiden Seiten des Gleichheitszeichens ohne es zu merken mit einer zusammengeseszten Grösse multipliciert hat, die in Wirklichkeit Null wird. Wenn man aus $A$ das richtige Resultat $K$ abgeleitet hat, so muss die Richtigkeit von $A$ dadurch geprüft werden, dass man womöglich die in der Aufgabe durchlaufene Schlussreihe zurück verfolgt, so dass also die Richtigkeit von $K$ diejenige von $A$ mit sich führt. Ist das der Fall, so liefert diese umgekehrte Schlussreihe den Beweis für die Richtigkeit von $A$, und man begnügt sich damit, diesen Beweis in der 
oben erwähnten synthetischen Darstellung mitzuteilen mit Auslassung der Analyse, die dazu geführt hat.

In dem Falle, wo das aus der Annahme $A$ abgeleitete Resultat falsch ist, kann man dagegen unmittelbar schliessen, dass auch $A$ falsch ist. Dies, oder, wenn $A$ und $B$ zwei Behauptungen sind, von welchen notwendig die eine richtig sein muss, die Behauptung, dass $B$ richtig ist, kamn man also als Theorem aufstellen und den Beweis dadurch führen, dass die Annahme, $B$ unrichtig oder $A$ richtig, zu dem unrichtigen Resultat $K$ führen würde. Ein solcher antithetischer Beweis ist apagogisch, also eigentlich analytisch. Da er indessen volle Sicherheit dafür gewährt, dass die Behauptung $B$ richtig ist, so wird er vielfach in Schriften gebraucht, in denen die Darstellung sonst synthetisch ist, häufig z. B. in Euklids Elementen. Die antithetische Beweisform wurde auch von Dinostratus auf die Quadratrix angewandt (S. 77) und wird, wie wir sehen werden, immer im Exhaustionsbeweise benutzt.

Ein Beispiel dafür, dass ein Theorem nicht aus einer Analyse des versuchsweise aufgestellten Theoremes selbst oder dessen Umkehrung hervorzugehen braucht, sondern aus der Analyse eines damit verbundenen Problemes, hatten wir dagegen in dem Theorem, das ausgeschaltet wurde, als wir oben versuchten die elliptische Flächenanlegung in zu grosser Allgemeinheit aufzustellen, dass nämlich ein Rechteck, das so an eine Strecke angelegt wird, dass ein Quadrat fehlt, nicht grösser ist als das Quadrat über der halben Strecke. Der synthetische Beweis für den etwas allgemeineren Satz bei Euklid VI, 27 wird in Übereinstimmung mit jener Analyse (S. 99) geführt. 
12. «Elemente»; analytische Hülfsmittel.

\section{"Elemente»; analytische Hülfsmittel.}

$\mathrm{Ob}$ man die Analyse benutzt um die Lösung einer Aufgabe oder den Beweis für einen Lehrsatz zu finden, oder ob man die Synthese benutzt um das gefundene darzustellen, immer ist die Lösung aus Lösungen von einfacheren Aufgaben zusammengesetzt und der Beweis auf der Richtigkeit von einfacheren Sätzen aufgebaut. Es wird also vorausgesetzt, dass man im voraus im Besitze von solchen ist. Um sich auf den hier beschriebenen Wegen vorwärts $\mathrm{zu}$ arbeiten, muss man im voraus eine Sammlung einfacherer Lösungen von Aufgaben und einfacherer Lehrsätze haben, die man als Ausgangspunkt benutzen kann. Die Werke, welche solche Sammlungen enthalten, heissen Elemente.

Die ersten Elemente, von denen berichtet wird, waren von Hippokrates geschrieben; aber leider kennen wir nicht dies aus so früher Zeit stammende Werk des erfindungsreichen und, wie es scheint, von den philosophischen Schulen ziemlich unabhängigen Geometers. Die reellen und formellen Fortschritte, die mittlerweile in den Schulen gemacht wurden, wurden später in neue Elemente aufgenommen. Einer von diesen Fortschritten, die Abgrenzungen oder Diorismen, werden dem nächsten Verfasser von Elementen, Leon, zugeschrieben, der sie also wohl mit in seine Elemente aufnahm. Seine und andere spätere Elemente sind verloren gegangen, nachdem diejenigen von Euklid die Alleinherrschaft gewonnen hatten, die sie während mehr als 2000 Jahren überall da behalten sollten, wohin die griechische Mathematik vorgedrungen war.

Mit diesem Hauptwerk wollen wir uns recht ausführlich beschäftigen. Es wird sich dann auch beim Studium dieses Werkes zeigen, wie solide es aus synthetisch dar- 
gestellten Problemen und Theoremen zusammengesetzt ist, und eine wie sichere Grundmauer es abgeben musste für die mathematischen Gebäude, die darauf aufgeführt wurden. Bei den hierzu führenden weitergehenden Untersuchungen konnte sich jedoch neben den durch und durch auf lo. gische Sicherheit berechneten Elementen das Bedürfnis nach Hülfsmitteln geltend machen, die eine für die analytische Arbeit bequemere Form besassen. Beispiele für darauf hinzielende Arbeiten lassen sich auch aus der Zeit vor und nach Euklid und ebenso von ihm selbst anführen. So soll Hermotimus, ein Nachfolger von Eudoxus, über geometrische Örter geschrieben haben, vermutlich über die sogenannten ebenen Örter, d. h. solche, die durch Gerade und Kreis dargestellt werden. Über denselben Gegenstand hat der grosse Geometer Apollonius später zwei Bücher geschrieben, über deren Inhalt Referate vorliegen, die in der neueren Zeit einen nicht geringen Einfluss auf die Bildung der analytischen Geometrie erhalten haben. Als ein Hülfsmittel bei der Anwendung der analytischen Methode haben wir ferner Euklids Data zu erwähnen. Dieses Werk geht, was seinen Inhalt betrifft, nicht über die «Elemente» hinaus, sondern teilt deren Inhalt in anderer Form mit. Die Sätze desselben gehen nämlich sämtlich darauf hinaus, dass, wenn gewisse Grössen oder Stücke einer Figur "gegeben» sind, gewisse andere es auch sind, d. h. durch die ersten bestimmt sind. Die ersten Sätze des Buches sagen aus, dass gegebene Grössen ein gegebenes Verhältnis, eine gegebene Summe u. s. w. haben, ein späterer, dass gegebene Geraden sich in einem gegebenen Punkte schneiden; andere stellen die Bedingungen dafür fest, dass ein Dreieck der Art nach gegeben ist, $d$. h. einem gegebenen ähnlich wird; noch andere teilen mit, dass zwei Grössen, deren Summe oder Differenz und deren Rechteck gegeben ist, selbst gegeben sind u. s. w. 
Die Bedeutung dieses Buches als analytisches Hülfsmittel ist einleuchtend. Es kam zunächst in der "Transformation» darauf an, aus der, eventuell mit Hülfslinien versehenen, Figur solche bekannte Stücke herauszufinden, die unbekannte Stücke bestimmen. Wenn man dann in der «Resolution» Auskunft darüber geben soll, dass man wirklich im Besitze des für die Lösung der Aufgabe Erforderlichen ist, so kann das dadurch allein geschehen, dass man die Sätze in der Form anführt, in der sie sich in den Data finden.

Die einzelnen Sätze in Euklids Data gewähren uns zugleich einen Einblick in einige der specielleren analytischen Methoden, die man zur Verfügung hatte. So ist in den Data nicht nur die Rede davon, welche Stücke ein Dreieck bestimmen können, sondern auch davon, welche nur seine Gestalt bestimmen. Es liegt nahe hieraus zu schliessen, dass man nicht nur Aufgaben dadurch löste, dass man in der Figur Dreiecke aufsuchte, mit deren vollständiger Konstruktion die Aufgabe beginnen konnte, sondern auch dadurch, dass man solche suchte, deren Gestalt allein bestimmt war. Die Konstruktion eines Dreiecks von dieser Gestalt konnte im allgemeinen nur der Ausgangspunkt sein für die vorläufige Kỏnstruktion einer Figur, die der gesuchten ähnlich war; hinterher würde dann die wirkliche Grösse einer oder der anderen Strecke einzuführen sein. In der griechischen Mathematik liegen in der That Aufgaben vor, die auf diesem Wege gelöst sind.

Die Zurückführung einer Aufgabe auf die Bestimmung zweier Grössen durch ihr Produkt (Rechteck) und ihre Summe oder Differenz, also auf die geometrische Lösung von Gleichungen zweiten Grades, wird durch die zuletzt angeführten Sätze aus den Data als eine brauchbare Methode hervorgehoben. Sie ist von den griechischen Mathe- 
matikern vielfach angewandt worden. Wir werden später sehen, dass andere Sätze der Data eine ähnliche Bekanntschaft mit verwickelteren Gleichungen verraten, die durch Proportionen und geometrische Algebra ausgedrückt sind.

\section{3. Überblick über Euklids Elemente; synthetisches Lehrgebäude.}

Euklids Elemente bestehen aus 13 Büchern, zu denen man in den meisten Ausgaben eine Arbeit von Hypsikles als 14tes, und eine jüngere und unbedeutendere Arbeit als 15tes Buch hinzugefügt hat.

Das erste Buch enthält die wichtigsten Sätze über Seiten und Winkel in Dreiecken, über Konstruktion von diesen, über senkrechte und parallele Geraden, über $\mathrm{Pa}$ rallelogramme und über ihren und der Dreiecke Flächen. inhalt. Das 2te Buch enthält die bereits benutzte Grundlage für die geometrische Algebra, das 3te die Lehre vom Kreise und von Linien und Winkeln im Kreise, auch den Potenzsatz. Das 4te Buch handelt von ein- und umbeschriebenen Vielecken, darunter namentlich von der Konstruktion des regelmässigen Dreiecks, Vierecks, Fünfecks, Sechsecks und Fünfzehnecks.

Euklids persönliche Arbeit an diesen Büchern ist wohl besonders darauf ausgegangen, diesen bekannten Stoff genauer als bisher geschehen darzustellen in Übereinstimmung mit den mittlerweile gestellten strengeren formellen Ansprüchen. Eine eigentliche mathematische Arbeit kann indessen auch damit verbunden gewesen sein. Die Proportionen wurden nämlich, wie wir bereits gesehen haben, in der Geometrie angewendet, auch bevor die exakte Proportionslehre des Eudoxus entstanden war. Wenn man dann in vielen Fällen gleichwohl seine $\mathrm{Zu}$ - 
flucht zu einer Proportionslehre nehmen musste, die nur auf der Lehre von rationalen Grössen aufgebaut war, so kam es nicht sonderlich darauf an, ob sie etwas früher oder später benutzt wurde. Euklid dagegen kannte Eudoxus' Lehre von den Proportionen. Diese war indessen zu neu, als dass sie ihren Platz am Anfange des Systemes hätte bekommen können, und musste deshalb bis zum 5 ten Buch aufgeschoben werden. Vorher musste also jede, offene oder versteckte, Benutzung der Proportionen und der Ähnlichkeit absolut vermieden werden. Es liegt z. B. nahe anzunehmen, dass es gerade diese Rücksicht war, die Euklid - wie früher berührt - dazu zwang, den Beweis für den pythagoreischen Lehrsatz zu erdenken, der sich am Schlusse seines ersten Buches findet. Um es verständlich $\mathrm{zu}$ machen, dass es überhaupt möglich war so weit ohne Proportionen zu gelangen, will ich daran erinnern, dass mit Hülfe der geometrischen Algebra die Sätze über die Potenz eines Punktes mit Bezug auf einen Kreis (III, 35-37) bewiesen worden waren. Diese Sätze werden benutzt um ein gleichschenkeliges Dreieck zu konstruieren, in dem der Winkel an der Spitze halb so gross ist wie ein Winkel an der Grundlinie (IV, 10); die Grundlinie ist dann Seite eines regelmässigen Fünfecks, das denselben umbeschriebenen Kreis hat wie dieses Dreieck (IV, 11).

Im 5ten Buch wird dann die Proportionslehre des Eudoxus dargestellt, und im 6ten Buch deren Anwendungen nicht nur auf die Geometrie, sondern, wie wir sehen werden, auch auf die Erweiterung der geometrischen Algebra. Die Konstruktion der mittleren Proportionale und die stetige Teilung einer Strecke, die im 2ten Buche in einer anderen Form mit Hülfe der geometrischen Algebra erhalten waren, kommen hier wieder vor, aber diesmal mit Hülfe der Proportionen abgeleitet, nämlich in 
VI, 13 und 30. Wie viele von den einzelnen Sätzen und Beweisen in diesen Büchern von Eudoxus herrühren, und wie viele früher mit einer weniger entwickelten Lehre von den Proportionen verknüpft waren, wissen wir nicht. Euklid gebührt sicher die Ehre dafür, alles zu einem systematischen Ganzen verarbeitet $\mathrm{zu}$ haben.

In dieses Ganze hat er jedoch nicht die specielle Lehre von rationalen Grössen und den ganzen Zahlen, durch deren Verhältnisse sie ausgedrückt werden, hineingearbeitet. Diese wird im 7ten bis 9ten Buch dargestellt, folgt also wohl auf die allgemeine Lehre von den Proportionen, ist aber nicht auf diese aufgebaut. Die Beweise sind wahrscheinlich dieselben, die man vor Eudoxus' Zeit benutzt hat, und von denen die Resultate damals auch auf irrationale Grössen übertragen wurden.

Die irrationalen Grössen selbst werden im 10ten Buch behandelt. Hier findet sich diejenige Klassifikation von ihnen, die Theätet begonnen hatte (vergl. S. 57), die aber von Euklid vollendet worden sein soll. Hier und bei der Anwendung der Klassifikation auf die Bestimmung der Stücke der regulären Polyeder findet sich wohl Euklids bedeutendste persönliche Arbeit.

Vor dieser Anwendung ist es jedoch nötig die elementare Stereometrie $\mathrm{zu}$ entwickeln. Das geschieht im 11ten Buch. Die Berechnung des Volumens der Pyramide verlangt infinitesimale Grenzbestimmungen; diese gewinnt man, wenn sie auch formell umgangen werden, durch den Exhaustionsbeweis von Eudoxus, der dafür im 12 ten Buch verwandt wird, nachdem er zuerst zu der zweiten in der elementaren Geometrie notwendigen Grenzbestimmung benutzt worden ist: zu dem Beweise dafür, dass zwei Kreise sich wie die Quadrate über ihren Durchmessern verhalten. Erst im 13ten Buch kommt die Bestimmung der Stücke der regulären Polyeder. 
Wie man sieht sind bis $\mathrm{zu}$ einem gewissen Grade gleichartige Stoffe, wie die Lehre von den irrationalen Grössen, und gleichartige Methoden, wie die Anwendungen des Exhaustionsbeweises, zusammengestellt. Zum Teil ist diese Zusammenstellung jedoch auf Rechnung der früheren historischen Entwickelung zu stellen, Euklid kann jedenfalls erst in zweiter Linie Rücksicht darauf nehmen. Mit Rücksicht auf die in der vorhergehenden Zeit entwickelten strengen logischen Principien, zu deren weiterer Verschärfung Euklid durch seine Schrift über Fehlschlüsse beigetragen hatte, war die logische Unanfechtbarkeit die Hauptsache. Während diese für das einzelne Problem oder Theorem, wie wir gesehen haben, durch die synthetische Darstellung verbürgt wurde, kam es darauf an, sowohl innerhalb jedes einzelnen Buches wie im ganzen Werke, die einzelnen Probleme und Theoreme so $\mathrm{zu}$ ordnen, dass die Grundlage, auf der, und das Material, aus dem jedes neue aufgeführt werden sollte, bereits durch die vorhergehenden hergestellt war. Mit Bezug hierauf haben wir angeführt, dass nicht einmal der Mittelpunkt einer Strecke in einem Beweis benutzt werden durfte, bevor nicht seine Existenz durch Konstruktion bewiesen worden war.

Eine solche Zusammenfügung von Sätzen, in der man, wie in einem synthetischen Beweise für einen einzelnen Satz, von dem Bekannten zu dem Unbekannten übergeht, sich also vom Einfachen und Einzelnen zum Zusammengesetzteren und Allgemeineren erhebt, wollen wir ein synthetisches Lehrgebäude nennen - ohne dass jedoch aus dem Altertum eine unmittelbare Berechtigung für diese Bezeichnung vorliegt. In einem solchen Lehrgebäude ist der Ausgangspunkt und der Schluss von besonderer Bedeutung. 
Was den Ausgangspunkt betrifft, so ist es klar, dass den Problemen, deren Lösungen aus solchen zusammengesetzt sind, die durch frühere Probleme gegeben wurden, und den Theoremen, deren Beweise auf frühere Theoreme und Probleme aufgebaut sind, gewisse er s te Konstruktionen vorangehen müssen, deren Ausführung ohne weiteres als bekannt betrachtet wird, und gewisse erste Behauptungen, deren Richtigkeit als unmittelbar einleuchtend angesehen wird. Die ersteren heissen bei Euklid Postulate oder

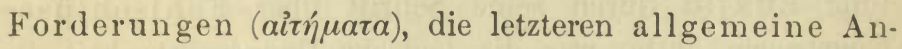

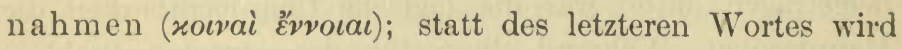
jedoch gewöhnlich das bei anderen, namentlich philosophischen Schriftstellern vorkommende Axiome ( $\dot{\xi} \xi \iota \dot{\omega} \mu \alpha \tau \alpha)$ genommen. Vor diesen beiden Arten von Voraussetzungen müssen die Begriffe aufgestellt werden, von denen diese Voraussetzungen gelten. Das geschieht in den Definitionen (ő@o ). Mit den Begriffen und Voraussetzungen, die in dieser Weise von Euklid aufgestellt werden, wollen wir uns demnächst beschäftigen. Dadurch lernen wir denn auch die Ansprüche kennen, die die Alten im ganzen an ihre Voraussetzungen stellten.

Ausser den Voraussetzungen zieht bei einem synthetischen Lehrgebäude auch der Schluss eine gewisse Aufmerksamkeit auf sich, da es bei der ganzen Anordnung den Anschein erhält, als ob alles Vorhergehende als Grund. lage für diesen Schluss mitgenommen ist. Wie bereits erwähnt schliessen Euklids Elemente mit der Bestimmung der Stücke der regulären Polyeder und der daraus folgenden Konstruktion der Polyeder. Dies ist ganz gewiss nicht Euklids einziges Ziel gewesen, denn im Verlauf seiner Arbeit nimmt er vieles mit, was weder direkt noch indirekt für diese Bestimmung benutzt wird; er hat also eine allgemeine Grundlage für weitergehende mathematische Untersuchungen hergestellt und sicherlich auch 
herstellen wollen. Die grosse Bedeutung, die der Konstruktion der regulären Polyeder als Schlussstein seiner Arbeit zukommt, ist jedoch die Veranlassung gewesen, dass Arbeiten anderer Schriftsteller über diese Polyeder bereits in sehr alte Ausgaben des Euklid als 14tes und 15 tes Buch aufgenommen sind.

In bestimmterer Weise geht die Behandlung im ersten Buche, für sich allein genommen, darauf aus, gerade das mitzubekommen, was logisch notwendig ist, un die erforderliche Grundlage für die in nächsten Buche entwickelte geometrische Algebra zu erreichen. Diese wird der Schlussstein des ersten Buches, nämlich der Satz vom Gnomon, I, 43, und der pythagoreische Lehrsatz, I, 47. Auch hier wird jedoch ein vorläufiges Ziel mitgenommen, das mitten im Buche in Verbindung mit der für das Hauptziel notwendigen Parallelentheorie erreicht wird, nämlich der Satz (32) über die Winkelsumme eines Dreiecks. Im übrigen enthält das Buch Sätze über die Lage gerader Linien gegen einander, über senkrechte und parallele Geraden mit zugehörigen Konstruktionen, über Kongruenz und Konstruktion ron Dreiecken, und über die Abhängigkeit zwischen Gleichheit und Ungleichheit ron Seiten und Winkeln in einer Vermengung, die nur wenig übersichtlich ist, die aber eine Folge ist ron der logisch wohl gesicherten Methode, nach der die Sätze allmählich auf einander aufgebaut sind. Beispielsweise wollen wir anführen, dass die Sätze über Kongruenz der Dreiecke sich in 4, 8 und 26 finden, und dass Euklid keine Veranlassung nimmt die Kongruenz von Dreiecken zu untersuchen, die in einem Winkel, einer anliegenden und einer gegenüberliegenden Seite übereinstimmen. Für Sätze hierüber hat er nämlich keine Verwendung; dagegen behandelt er im 6ten Buche, wo er Sätze über die Ähnlichkeit der Dreiecke zusammenstellt, auch den hierher gehörigen 
Fall. Am Schlusse des Buches sind die Sätze über Flächengleichheit mehr zusammengerückt.

Da wir hier den Begriff synthetisches Lehrgebäude aufgestellt haben, so wollen wir des Gegensatzes wegen - und da wir auch, abgesehen von der Anwendung auf die Schriften der Alten, wünschen $\mathrm{zu}$ voller Klarheit über die Begriffe Analyse und Synthese zu gelangen - berühren, was wir unter einem analytischen Lehrgebäude verstehen würden. Während man sich in dem synthetischen Lehrgebäude erst allmählich zu der Betrachtung von zusammengesetzteren und allgemeineren Verhältnissen erhebt, nimmt man im analytischen Lehrgebäude ein allgemeines Princip, dass gerade durch seine Allgemeinheit eine gewisse Einfachheit besitzen kann, zum Ausgangspunkt und entwickelt von diesem aus die Verhältnisse, die in den verschiedenen einzelnen Fällen zur Geltung gelangen müssen. Eine Behandlung der Geometrie, bei der man mit der geraden Linie und dem Kreise anfängt und sich demnächst durch die Kegelschnitte zu Kurven höheren Grades erhebt, ist ihrer ganzen Anlage nach synthetisch, selbst wenn die Einzelheiten analytisch behandelt werden; eine Behandlung aber, bei der man sofort die Eigenschaften allgemeiner Kurven untersucht und daraus im besonderen Sätze über die Gerade oder die Kegelschnitte ableitet, ist ihrer Anlage nach analytisch. Ein ausgeprägtes Beispiel für eine analytische Behandlungsweise besitzen wir in der analytischen Mechanik von Lagrange, in der alles aus dem Princip der virtuellen Geschwindigkeiten abgeleitet wird. Wäre dies Princip selbst nur als eine Hypothese aufgefasst, die durch ihre Anwendungen oder Konsequenzen bewiesen werden sollte, so würde das Verfahren ganz übereinstimmen mit der bereits erwähnten Anwendung der analytischen Methode auf einzelne Sätze. Ist das Princip dagegen wie bei Lagrange im voraus sicher gestellt, so ist das angewandte Verfahren dennoch wesentlich dasselbe, und wenn man es analytisch nennt, so stimmt das immer noch zu der früher gebrauchten Anwendung dieses Wortes. Im übrigen wird in der Regel durch ein synthetisches Fortschreiten vom Specielleren zum Allgemeineren der allgemeine Gesichtspunkt gewonnen werden, der der Ausgangspunkt für ein analytisches Lehrgebäude ist. 


\section{Euklids geometrische Voraussetzungen.}

Die Voraussetzungen, auf denen Euklid die Geometrie aufbaut, muss man in den Definitionen, Postulaten und Axiomen suchen, die an der Spitze seiner verschiedenen Bücher stehen. Besonderes Interesse haben diejenigen, die zum ersten Buche gehören, da sie und die nach und nach darauf aufgebauten Resultate auch den folgenden zu Grunde liegen. Bei diesen wollen wir deshalb hier namentlich verweilen, sie jedoch sofort durch einige der in andere Bücher eingeführten neuen Voraussetzungen ergänzen. Diejenigen, die in Verbindung mit besonderen Theorien, wie mit der Lehre von den Proportionen, stehen, wollen wir jedoch erst in Verbindung mit diesen Theorien besprechen.

Bei dem ersten Durchlesen von Euklids Definitionen, Postulaten und Axiomen wird man gewiss finden, dass sie keineswegs auf gleicher Höhe mit den formellen und logischen Ansprüchen stehen, die die Alten nach unserer Aussage erhoben haben. Beispielsweise wird man sehen, dass verschiedene von den Definitionen gar nichts von dem sagen, was definiert werden soll, und keine Sicherheit dafür gewähren, dass wirklich etwas existiert, was den Definitionen entspricht. Die Definition der geraden Linie sagt nichts mehr, als wenn man gesagt hätte: es giebt eine gewisse Art von Linien, die gerade heissen. Was es für Linien sind - also die Eigenschaften der Linien, die man in unseren Tagen für die Definition benutzen würde - das wird erst in den Postulaten ausgesagt, die also sagen: wir wollen davon ausgehen, dass die gerade Linie die und die Eigenschaften hat. Auch die Postulate und Axiome sind zum Teil mit einer Kürze ausgedrückt, die sie rätselhaft erscheinen lassen kann und einen starken Gegensatz zu der vorsichtigen Ausführ- 
lichkeit bildet, mit der alles in den eigentlichen mathematischen Sätzen und ihren Beweisen behandelt wird.

Die Sache ist die, dass in die Definitionen, Postulate und Axiome alles das verwiesen wird, zu dessen Voraussetzung im Lehrgebäude der Mathematiker berechtigt sein will, ohne weder eine Erklärung oder Beschreibung vom «wie», oder eine Begründung vom "weshalb» $z u$ geben. Es ist seine Sache im voraus ein genaues Verzeichnis von dem zu geben, was er voraussetzen will, und dieses muss so deutlich sein, dass es, sobald er es benutzen soll, klare Auskunft giebt, so dass es dann klar wird, dass er weder mehr noch weniger gebraucht als das, wozu er sich das Recht ausbedungen hat; die Abstraktionen aber, die dazu geführt haben die Begriffe aufzustellen und ihnen in Postulaten und Axiomen gerade diese bestimmten Eigenschaften beizulegen, ja selbst ein vorläufiger Nachweis darüber, dass er wirklich die Forderung erfüllt habe, ihnen weder zu viele noch $\mathrm{zu}$ wenige Eigenschaften beizulegen, gehen ihn nichts an. Er ist als Mathematiker nur verantwortlich dafür, dass derjenige, der ihm alle diese Dinge einräumt, hinterher durch seine sicheren Schlüsse gezwungen werden muss alles das einzuräumen, was er daraus ableitet. Dabei muss es sich praktisch zeigen, dass er eine hinreichende Anzahl von Voraussetzungen gemacht hat. Dass er nicht zu viele gemacht hat, lässt sich wohl kaum so unmittelbar nachweisen; aber wenn er es gethan hätte, so würde er sich dem aussetzen, dass andere ihm nachweisen könnten, dass er es gethan hätte, nämlich dadurch, dass einige von den Voraussetzungen in Widerstreit mit einander wären oder aus einander abgeleitet werden könnten.

Wenn man die von den Alten, namentlich von $\mathrm{Eu}$ klid, ausdrücklich aufgestellten geometrischen Voraussetzungen richtig würdigen will, so muss man mehr dar- 
auf sehen, welches diese Voraussetzungen sind, als darauf, dass Angaben darüber fehlen, woher sie stammen, oder auf die Form, unter der sie auftreten. Dann wird sich zeigen, dass es dieselben sind wie diejenigen, auf denen wir noch heutigen Tages die Geometrie aufführen, und dass sie mit einer Sicherheit und Vollständigkeit vorgeführt werden, die dauernd auch denen zum Muster dienen muss, die Veranlassung zu einzelnen Ergänzungen oder Modifikationen finden könnten. Um sie ganz zu verstehen, müssen wir jedoch dann und wann auch die, wenigstens für eine moderne Auffassung, mangelhaften Formen berühren, in denen mehrere von ihnen auftreten.

Wir wollen damit beginnen diejenigen Definitionen hervorzuziehen, die uns Veranlassung zu einigen Bemerkungen geben können. Der Punkt wird durch seine Unteilbarkeit definiert (I, Def. 1.). Von da aus geht es weiter zu der Linie als Länge ohne Breite (I, 2), zu der Fläche mit Länge und Breite $(I, 5)$ und im 11ten Buche zum Körper mit Länge, Breite und Dicke (XI, 1). Diese Definitionen geben keine Aufklärung darüber, wie man zu den Begriffen Punkt, Linie, Fläche und Körper gelangen soll, nennen also als eine Voraussetzung, auf der man bauen soll, dass man bereits im Besitze dieser Begriffe ist und versteht, was es heissen soll, dass der Punkt 0 Dimensionen hat, die Linie $1 \mathrm{u}$. s. w.; hierin liegt wieder, dass eine Linie als ein geometrischer Ort für Punkte, Fläche und Körper als solche für Linien und Flächen aufgefasst werden. Um wirklich in den Besitz der Begriffe $\mathrm{zu}$ gelangen, wird man in der Regel nicht diesen synthetischen Weg von Punkt zu Linie, Fläche und Körper gehen, sondern den umgekehrten analytischen, indem man also vom Körper als etwas unmittelbar gegebenem ausgeht, die Fläche als eine Grenze für den Körper betrachtet u. s. w. Dass dieser Weg zu den Begriffen zu 
gelangen den Alten nicht unbekannt war, sieht man aus einer anderen Reihe von Definitionen, nämlich $\mathrm{XI}$, 2, I, 6 und I, 3, die indessen bei Euklid nicht neue Definitionen von Fläche, Linie und Punkt sind, sondern nur Angaben darüber, wie Körper, Fläche und Linie begrenzt werden.

Dass man nicht in den Definitionen, sondern erst in den Postulaten in Verbindung mit einem der Axiome Aufklärung darüber suchen muss, was eine gerade Linie ist, habe ich bereits berührt. Auch die Existenz der Kreislinie wird erst sichergestellt in den Postulaten, wogegen ihre Definition ( $I, 15)$ eher $\mathrm{zu}$ viel als $\mathrm{zu}$ wenig zu sagen scheint. Diese berichtet nämlich nicht nur, dass alle Punkte der Kreislinie dieselbe Entfernung vom Centrum haben sollen, sondern giebt zugleich an, dass der Kreis selbst eine Figur ist, also ein durch die Kreislinie abgegrenzter Teil der Ebene, und dass das Centrum innerhalb dieser Linie liegt. Wenn nicht gesagt ist, dass die Kreislinie alle Punkte von der zuerst angeführten Eigenschaft enthalten soll, so wird die erste der angeführten Angaben immerhin kein überflüssiges Unterscheidungsmerkmal zwischen der ganzen Kreislinie und dem Kreisbogen. Dadurch kann sie ihren Platz zwischen den Definitionen behaupten. Im übrigen werden wir sehen, dass diese Angaben, wenn sie ihren Platz nicht hier erhalten hätten, in einer oder der anderen Form unter die Postulate aufgenommen werden müssten.

Die Definition eines Durchmessers im Kreise (I, 17) enthält dagegen einen Zusatz, der sicher überflüssig ist, nicht nur für die Definition, sondern überhaupt unter den Voraussetzungen. Es wird nämlich nicht nur gesagt, dass der Durchmesser durchs Centrum geht, sondern auch, dass er den Kreis halbiert. Dies letztere ist ein Satz, der sich durch die Kongruenz der beiden Teile, in die 
der Kreis geteilt wird, beweisen lässt. Vielleicht hat ein späterer Herausgeber ihn in die Definition hineingeschoben, weil er sich in der That in keinem Lehrsatz bei Euklid findet.

Euklids Definition eines Winkels ist an sich beinahe ebenso leer wie die Definition der geraden Linie. Dem wird jedoch abgeholfen durch die Axiome, in denen allgemeine Kennzeichen dafür aufgestellt werden, ob eine geometrische Grösse grösser, gleich, oder kleiner ist als eine andere derselben Art. Diese Kennzeichen lassen sich nämlich auch auf Winkel anwenden, und da es sich zugleich zeigt, dass Winkel addiert werden können, so erhalten sie dadurch wohl definierte Grössen (vergl. im Folgenden S. 127). Im übrigen sei bemerkt, dass die ursprüngliche Definition des Winkels auch anwendbar ist auf Winkel zwischen krummen Linien. Benutzt wird dieser Begriff in III, 16, wo gezeigt wird, dass die Senkrechte auf dem Durchmesser in einem Punkte der Kreisperipherie einen kleineren Winkel mit dem Kreise bildet oder ihm näher kommt als jede andere gerade Linie.

Die Postulate, die Euklid im ersten Buche aufstellt, sind nach der neuesten und zuverlässigsten Textrevision ${ }^{1}$ folgende:

1. Eine gerade Linie von einem Punkte bis zu einem anderen $\mathrm{zu}$ ziehen.

2. Eine begrenzte gerade Linie unbegrenzt zu verlängern.

3. Einen Kreis mit gegebenem Mittelpunkt und gegebenem Radius zu beschreiben.

4. Alle rechten Winkel sind unter sich gleich.

${ }^{1}$ Euclidis Elementa; edidit et latine interpretatus est J. L. Heiberg. Lipsiae 1883-88, $8^{\circ}$. 
5. Wenn eine gerade Linie, die zwei andere gerade Linien schneidet, auf derselben Seite innere Winkel bildet, die zusammen kleiner als zwei Rechte sind, so werden die letzgenannten beiden Linien bei der Verlängerung bis ins Unendliche sich auf der Seite schneiden, wo die Winkelsumme kleiner als zwei Rechte ist.

Die Konstruktionen, aus denen sich alle übrigen nach diesen Postulaten sollen zusammensetzen lassen, sind diejenigen, die praktisch durch Zirkel und Lineal ausgeführt werden. Indessen würde es irreführend sein, nur dies einseitig festhalten zu wollen. Das zeigt sich unter anderem dadurch, dass dann kein rechter Platz mehr bleiben würde für die beiden letzten Postulate, weshalb denn auch bereits sehr frühe Herausgeber sich haben verleiten lassen diese unter die Axiome $\mathrm{zu}$ versetzen.

Wie man sieht, werden Lineal und Zirkel gar nicht genannt. Durch ihre Benutzung würde man ja auch nur ein unvollständiges Bild von der mathematischen geraden Linie und dem mathematischen Kreise geben. Selbst die drei ersten Postulaten geben, wie wir bereits über Euklids Voraussetzungen überhaupt gesagt haben, gar keine Aufklärung darüber, von wo aus oder durch welche Mittel man das zustande bringt, was man vorausgesetzt haben will. In Übereinstimmung damit, dass die Probleme der Alten im wesentlichen Sätze über die Existenz, und ihre Lösungen Beweise für die Existenz des Behandelten oder Gesuchten sind, sind die Postulate Behauptungen über dessen Existenz, deren Anerkennung ohne Beweis oder Nachweis verlangt wird. Die Behauptungen, die in den drei ersten Postulaten enthalten sind, wollen dann nur sagen, dass es eine gerade Linie durch zwei beliebige gegebene Punkte giebt, dass diese ohne Grenze verlängert werden kann, und dass es einen Kreis giebt mit einem beliebigen gegebenen Mittelpunkt und einem beliebigen 
gegebenen, von diesem ausgehenden Radius, oder mit anderen Worten einen Kreis, der einen gegebenen Mittelpunkt hat und durch einen gegebenen Punkt geht. Dass das dritte Postulat wirklich so $\mathrm{zu}$ verstehen ist und nicht etwa verlangt, dass die Existenz eines Kreises mit gegebenem Mittelpunkt und einem an einer Stelle der Ebene gegebenen Radius ohne Beweis eingeräumt werde, ergiebt sich sofort daraus, dass Euklid im 2ten Satze zeigt, dass die Bestimmung eines solchen Kreises mit Hülfe der im ersten Satze mitgeteilten Konstruktion eines gleichseitigen Dreiecks sich aus den postulierten Konstruktionen zusammensetzen lässt. $\mathrm{Da}$ dies sich wirklich thun lässt, so hat Euklid durch die angeführte Einschränkung des 3ten Postulates nur die bereits genannte Pflicht erfüllt nicht zuviel vorauszusetzen. Wäre dagegen nur die Rede von der praktischen Ausführung mittels des Zirkels, so wäre die Lage des gegebenen Radius gleichgültig gewesen, und man darf wohl sagen, dass der im 2 ten Satze angegebene Weg nicht für die praktische Ausführung von Zeichnungen bestimmt gewesen ist.

Bei dieser Auffassung von der Bedeutung der Postulate ist es offenbar, dass es nicht genügt, die Existenz der auf die einfachste Weise bestimmten geraden Linien und Kreise zu postulieren. Die geometrischen Konstruktionen werden dadurch ausgeführt, dass man mittels des Durchschnittes verschiedener Linien Punkte bestimmt, die wieder zur Bestimmung von neuen Linien benutzt werden können. Dann muss die Existenz der Schnittpunkte ebensowohl wie diejenige der Linien postuliert werden, denn sie kann unmöglich eine Folge dieser letzteren sein. Im 5ten Postulat wird es deshalb ausdrücklich als eine neue Voraussetzung aufgestellt, dass zwei gerade Linien sich schneiden, wobei jedoch die Einschränkung gemacht werden muss, die notwendig ist, damit die Behauptung wirk- 
lich wahr werden kann, eine Einschränkung, die hier ganz dieselbe Rolle spielt, wie der Diorismus $z u$ einem Problem. Wenn nicht die Existenz des Schnittpunktes im 5ten Postulat verlangt worden wäre, so würden die Lösungen der Probleme, bei denen Schnittpunkte zwischen geraden Linien benutzt werden, im Allgemeinen durchaus nicht diejenigen Beweise für die Existenz der konstruierten Figuren geben, die die wesentliche Ausbeute der Konstruktionen sein sollten.

Ist diese Betrachtung richtig, so wird man Postulate vermissen, die auf ähnliche Weise die Existenz von Schnittpunkten zwischen der geraden Linie und dem Kreise oder zwischen zwei Kreisen anerkennen. Allerdings muss die vollständige Abgrenzung der Fälle, in denen das Durchschneiden wirklich stattfindet, bereits die Entwickelung von mehreren Sätzen verlangen, und vielleicht hat der Umstand, dass Euklid deshalb nicht sofort diese Abgrenzung in voller Allgemeinheit geben kann, ihn abgehalten die hierzu dienenden Forderungssätze aufzustellen. Um überhaupt den Kreis bei Konstruktionen benutzen zu können, sind jedoch wenigstens einige Voraussetzungen über sein Schneiden mit der geraden Linie und mit anderen Kreisen notwendig. Welche Euklid benutzt, das muss man in den Anwendungen suchen, die er macht.

Da sieht man demn in Satz I, 12, dass er um sicher zu sein, dass ein Kreis mit gegebenem Mittelpunkt eine gewisse gerade Linie schneidet, diesen Kreis dureh einen Punkt gehen lässt, der auf der dem Mittelpunkt entgegengesetzten Seite der Geraden liegt, und dass er es in Satz 1 als einleuchtend betrachtet, dass zwei Kreise, jeder mit dem Mittelpunkt auf der Peripherie des anderen, sich in zwei Punkten schneiden, und in Satz 22, dass auch ein Kreis, der sowohl dureh einen Punkt innerhalb als durch einen Punkt ausserhalb der Peripherie eines anderen Kreises 
geht, diesen anderen Kreis schneidet. Dass er sich auf diese Voraussetzungen stützt, geht aus den betreffenden Stellen hervor, und an anderen Stellen wird nichts über das Durchschneiden zwischen Kreis und gerader Linie oder Kreis vorausgesetzt, bevor das dazu erforderliche bewiesen ist.

Enthalten die von Euklid ausdrücklich aufgestellten Voraussetzungen denn gar nichts über diese faktischen Voraussetzungen, deren Euklid sich an den angeführten Stellen, namentlich in Satz 12, vollkommen bewusst ist? Die Postulate thun es jedenfalls nicht, aber, wie wir gesehen haben, sind die Untersehiede zwischen Postulaten und Definitionen nicht so ausgeprägt, dass man allein zwischen den ersten zu suchen braucht. Dann ist es klar, dass Euklid die Berechtigung zur Benutzung dieser Voraussetzungen darin suchen kann, dass er in den Definitionen gesagt hat, ein Kreis sei eine Figur, die den Mittelpunkt enthält, woraus dann folgen muss, dass eine Kreislinie eine hinreichend verlängerte gerade Linie in zwei Punkten schneiden muss, wenn sie ihren Mittelpunkt auf der einen Seite von dieser Linie hat und durch einen Punkt auf der anderen Seite geht, und ebenso eine andere Kreislinie, wenn sie einen ausserhalb liegenden Punkt mit einem inneren verbindet. Im übrigen sei bemerkt, dass man auf ähnliche Weise in gewissen Fällen das Durchschneiden gerader Linien ohne Benutzung des 5ten Postulates beweisen kann, wenn man berücksichtigt, dass die Umfänge von Polygonen auch Flächen abgrenzen, die keine unendliche Ausdehnung haben. Davon macht Euklid in I, 21 Gebrauch.

Noch fehlt uns eine Erklärung dafür, wie die Behauptung, dass alle rechten Winkel gleich gross sind, ihren Platz unter den Postulaten erhalten kann. Aus den Axiomen geht hervor, dass alle Winkel gleich gross sind, wenn sie kongruent sind, sonst nicht, und die Behauptung ist also 
genau dieselbe wie die andere, dass alle rechten Winkel kongruent sind. Da ein rechter Winkel (in Def. 10) als solcher definiert wird, der seinem Nebenwinkel gleich ist, so läuft das Postulat darauf hinaus, dass der Winkel, den wir jetzt einen gestreckten nennen, eine bestimmte Grösse hat, oder dass die Verlängerung einer gegebenen geraden Linie über den einen Endpunkt hinaus eindeutig bestimmt ist. Eine volle Bestätigung dafür, dass dies gemeint ist, erhält man, wenn man sieht, dass das Postulat gerade auf diese Weise faktisch angewandt wird; das geschieht im Beweise für den Satz I, 14.

Das 4te Postulat wird also ein Zusatz zum 2ten, dass nämlich die in diesem enthaltene Bestimmung der Verlängerung einer geraden Linie eindeutig ist, und es hat wohl zunächst deswegen seinen Platz unter den Postulaten und nicht unter den Axiomen erhalten. Das Postulat würde nicht vermisst werden von einem modernen Leser, der gewohnt ist, dass auf die Anzahl der Lösungen Rücksicht genommen wird, und sich deshalb zunächst denken würde, dass die Eindeutigkeit bereits im 2ten Postulat mit unterverstanden ist. Wenn es nun doch einmal dasteht, so vermisst man ein anderes Postulat, welches ausdrückt, dass auch die in dem ersten Postulat gegebene Bestimmung einer geraden Linie eindeutig ist. Von dieser Eindeutigkeit macht. Euklid ausdrücklich Gebrauch in Satz I, 4, wo er in seiner Beweisführung das Argument gebraucht, dass "zwei gerade Linien keinen Flächenraum einschliessen können»; aber diese Behauptung, die durchaus zusammenfällt mit derjenigen, dass das erste Postulat eindeutig ist, findet sich nicht unter den aufgestellten Voraussetzungen. Hier liegt unzweifelhaft eine Inkonsequenz vor. Auf diese ist man schon im Alterturn aufmerksam geworden und sie hat die Herausgeber veranlasst, die in I, 4 ausdrïcklich benutzte Voraussetzung entweder 
- und wahrscheinlich am frühesten - unter die Postulate aufzunehmen, wohin sie mit demselben Recht gehört wie das Postulat I, 4, oder unter die Axiome. Dieses neue Postulat drückt zugleich aus, dass die Bestimmung eines Punktes mittels des 5ten Postulates als Schnittpunkt zwischen zwei Geraden eindeutig ist.

Die Eindeutigkeit des 3ten Postulates über die Bestimmung eines Kreises durch Mittelpunkt und Radius braucht man dagegen nicht vorauszusetzen. Man kann hier nämlich wieder davon Gebrauch machen, dass der Kreis bereits in den Definitionen vollständiger bestimmt ist als die gerade Linie. Dadurch ist Euklid imstande in den Sätzen III, 5 und $6 \mathrm{zu}$ beweisen, dass koncentrische Kreise sich nicht schneiden oder berühren können, dass also der vollständige geometrische Ort für die Punkte, die denselben Abstand von einem gegebenen Punkte haben wie ein anderer Punkt, núr aus einer geschlossenen Kurve besteht, mit anderen Worten, dass das 3te Postulat nur einen Kreis giebt.

Euklids 1stes, 2tes, 4tes und 5tes Postulat, ergänzt durch die in Satz I, 4 benutzte Voraussetzung, dass das erste Postulat eine eindeutige Bestimmung geben soll, und, wie wir sehen werden, durch eine im 7 ten Axiome enthaltene Voraussetzung, drücken alle Eigenschaften einer geraden Linie aus, welche ihrer Verwendung in der Geometrie zu Grunde liegen. Unvermerkt, ja, wie wir sehen werden, ohne es selbst gewahr worden zu sein, hat Euklid damit indes gleichzeitig die Grundeigenschaften der Ebene aufgestellt erhalten. Die ausdrücklich aufgestellte Definition der Ebene (I, 7) ist an sich ebenso nichtssagend wie diejenige der geraden Linie. Die Ebene wird noch in den Definitionen I, 8 und 15 genannt, wo ausgesprochen wird, dass die Schenkel eines Winkels in derselben Ebene liegen müssen, und dass der Kreis eine ebene 
Figur ist. Von grösserer Bedeutung ist es, dass in den aufgestellten Postulaten stillschweigend vorausgesetzt wird, dass die verschiedenen Bestimmungen innerhalb einer und derselben Ebene stattfinden. Ohne dieses würde das 5te Postulat geradezu sinnlos sein. Die Eigenschaft, die namentlich durch das erste und zweite Axiom einer Ebene beigelegt wird, wird nun die, dass sie jede gerade Linie, die durch zwei ihrer Punkte geht, nebst ihren Verlängerungen bis ins Unendliche ganz enthält. Hätte Euklid selbst dies ausdrücklich festgestellt, so hätte er darin eine wirkliche Grundlage erhalten können für die drei ersten Sätze des 11ten Buches, die aussagen, dass eine gerade Linie, die teilweise in einer Ebene liegt, nicht aus dieser herausgehen kann, dass zwei gerade Linien, die sich schneiden, in einer Ebene liegen (und sie bestimmen), und dass die Durchschnittslinie zweier Ebenen gerade ist. Nun stellt er einige andere Beweise auf, von denen der für XI, 1 die Richtigkeit von XI, 2 voraussetzen muss, der umgekehrt wieder auf XI, 1 aufgebaut ist. In logischer Beziehung, sowohl principiell wie formell, steht Euklids Behandlung der Stereometrie im ganzen hinter seiner ebenen Geometrie zurück, wofür wir ein noch wichtigeres Beispiel bei der Besprechung seiner Axiome sehen werden. Indessen wird sich zeigen, dass die griechischen Mathematiker trotz dieses Mangels die stereometrischen Sätze und Operationen dennoch in einem recht bedeutenden Umfange kannten.

Während wir bei Definitionen und Postulaten, um genaue Auskunft über die Voraussetzungen zu erhalten, die sie ausdrücken sollen, zum Teil unsere Zuflucht zu den Anwendungen haben nehmen müssen, die Euklid in seinen Sätzen faktisch von ihnen gemacht hat, so geben diejenigen Axiome des ersten Buches, deren Ächtheit für 
unzweifelhaft angesehen wird und mit denen wir uns deshalb ausschliesslich beschäftigen wollen, nämlich $1-3$ und $7-8^{1}$, eine ebenso kurze wie klare Auskunft über die Grundlage für die Anwendung der Begriffe Gleichheit und Ungleichheit auf Grössen im allgemeinen, und auf geometrische Grössen im besonderen. Der erste Beitrag zum Begriffe Gleichheit wird im Axiom 1 gegeben: Grössen, die einer und derselben Grösse gleich sind, sind unter sich gleich. Der Umstand, dass das Wort "gleich» in der Erklärung des Begriffes Gleichheit vorkommt, macht diese Erklärung nicht wertlos, was unter anderem daraus ersichtlich ist, dass man nicht in der im Axiome enthaltenen Erklärung «ungleich» an die Stelle von "gleich» setzen kann. Sie ist indessen nicht ausreichend um einen anwendbaren Grössenbegriff geben zu können. Es muss hinzugenommen werden, dass eine Grösse nicht verändert wird durch Teilung und eine darauf folgende Zusammensetzung aus allen Teilen. Dies ist in den Axiomen 2 und 3 enthalten, die aussagen, dass Gleiches, um Gleiches vermehrt oder vermindert, Gleiches giebt. Ferner muss, wenn man auch die Ungleichheit berücksichtigen will, hinzugenommen werden, dass man etwas Kleineres erhält, wenn man nicht alle Teile mitnimmt; das wird im Axiom 8 ausgesagt: das Ganze ist grösser als ein Teil. Hierdurch ist auch eine Erklärung von Addition und Subtraktion allgemeiner Grössen gegeben, und zugleich ist darin enthalten, dass die Reihenfolge der Summanden gleichgültig ist. Wenn der Grössenbegriff in einer modernen Arithmetik ${ }^{2}$ so definiert wird, «dass von denjenigen Eigenschaften der Gegenstände, die sich nicht

${ }^{1}$ Euclidis Elementa ed. Heiberg, Lipsiae 1883-88.

2 Julius Petersen, Arithmetik og Algebra til Skolebrug, Kjøbenhavn 1879, S. 3. 
verändern, wenn ihre Teile in verschiedener Reihenfolge zusammengesetzt werden, sich aber verändern, wenn einige der Teile fortgenommen werden, gesagt wird, sie besässen Grösse», so stimmt diese Definition durchaus zu den angeführten Axiomen, die sogar den Vorzug haben etwas direkter zu erklären, was gleich, grösser oder kleiner ist.

Dieser allgemeine Grössenbegriff muss durch besondere Kennzeichen ergänzt werden, die seine Anwendung ermöglichen sowohl auf bestimmte Arten von Grössen, wie geometrische Grössen, Gewichte u. s. w., als auch auf rein abstrakte Zahlengrössen. Euklid, für den die geometrische Grösse zur abstrakten Grösse wird, da sie in der geometrischen Algebra zur Darstellung von Grössen jeder Art, auch von Zahlen, dient, muss zuallererst Kennzeichen für die Gleichheit geometrischer Grössen geben; das geschieht im 7 ten Axiom zum ersten Buch, von dem wir jetzt gleich reden wollen. Erst im 5ten Buche wird eine unmittelbare Darstellung abstrakter Grössen als Verhältnisse gegeben und ausserdem Kennzeichen für ihre Gleichheit und Ungleichheit. Die Verhältnisse zur Einheit sind Zahlen in der modernen und allgemeinen Bedeutung dieses Wortes. Die Einheit wird jedoch erst im 7ten Buch eingeführt und dann nur als Maass für damit kommensurable Grössen angewandt. Die dazu dienenden Voraussetzungen werden wir im Zusammenhang mit dem übrigen Inhalt dieser Bücher besprechen.

Im 7 ten Axiom des ersten Buches, zu dem wir nun. mehr nach diesem Ausblick auf andere Grössenbestimmungen als die geometrische zurückkehren, wird ausgesprochen, dass kongruente Grössen oder solche, die sich zur Deckung bringen lassen, gleich sind. Dieses Kennzeichen für geometrische Gleichheit geht ganz natürlich dem im 8ten Axiome enthaltenen Kennzeichen für Ungleichheit voran, das keine besondere Ergänzung mit Bezug 
auf die geometrischen Grössen bedarf. Euklid weist im 7 ten Axiom mit grosser Sicherheit auf das hin, was immer der erste Ausgangspunkt für jede Untersuchung von Grössen in der Geometrie sein muss. Die Kongruenz ist es bereits beim praktischen Messen, das darin besteht, nach und nach von dem, was gemessen werden soll, eine Reihe von Teilen aufzuzählen, die dem Maasse kongruent sind. Sie ist es ferner sowohl in Euklids eigenem Lehrgebäude wie in allen folgenden, die geometrische Grössen behandeln: man geht davon aus, dass gewisse Grössen gleich sind, weil sie kongruent sind, und ungleich, weil die eine selbst ein Teil der anderen ist oder einem Teile der anderen kongruent ist. Eben dieses Verfahren wendet Euklid im ersten Buche an um zu zeigen, wie Gleichheit und Ungleichheit von Seiten oder Winkeln desselben Dreiecks oder verschiedener Dreiecke sich gegenseitig bedingen. Die Resultate hiervon kombiniert er dann mit den allgemeinen Voraussetzungen über Grössen. Ja er ist sogar bestrebt das specifisch geometrische Princip der Kongruenz so wenig wie möglich anzuwenden. So benutzt er in I, 26 nicht unmittelbar die Kongruenz um zu beweisen, dass Dreiecke, die in einer Seite und den anliegenden Winkeln übereinstimmen, es auch in den übrigen Stücken thun, sondern er schliesst dies antithetisch aus den früheren Kongruenzfällen.

Für gerade Linien und Winkel fällt Gleichheit zusammen mit Kongruenz. Für gebrochene Linien, Flächenund Rauminhalte kann man dagegen erst, nachdem man Gleichheit durch Kongruenz nachgewiesen hat, Gleichheit ohne Kongruenz nachweisen durch Zusammensetzung nach den allgemeinen Voraussetzungen über Grössen, wie es beispielsweise in I, 35 geschieht, wo bewiesen wird, dass Parallelogramme von derselben Grundlinie und Höhe gleich sind. $\mathrm{Zu}$ der Grösse krummer Linien und der 
Flächen, die von solchen begrenzt werden, sowie zu der Grösse der meisten Rauminhalte kann man nur durch Grenzübergänge gelangen, die von den Alten durch den Exhaustionsbeweis ausgeführt wurden, und die zum Teil zugleich die Aufstellung neuer Voraussetzungen verlangen. Das werden wir sehen, wenn wir dazu gelangen Euklids 12 tes Buch, sowie die Arbeiten von Archimedes zu besprechen.

Dagegen müssen wir sogleich erwähnen, dass die Art und Weise, wie Euklid in der Stereometrie das im 7 ten Axiom aufgestellte Kongruenzaxiom benutzt, mit einem sehr wesentlichen Mangel behaftet ist. Dieser hängt damit zusammen, dass man in seiner Stereometrie jedes Unterscheiden zwischen Kongruenz und Symmetrie vermisst, lässt aber doch erkennen, dass er symmetrische Figuren nicht für kongruent hält. Denn in solchem Falle würde er gemeint haben im 7 ten Axiom eine ausreichende Grundlage für Volumenbestimmungen zu besitzen. Statt dessen stellt er eine neue Voraussetzung auf, die sowohl auf kongruente wie auf symmetrische Figuren passen kann. In der 10ten Definition des 11ten Buchs werden gleiche und ähnliche Raumfiguren als solche definiert, die von gleichvielen gleichen und ähnlichen (d. h. kongruenten) ebenen Figuren eingeschlossen werden. Diese Definition enthält ausser einer Namengebung zugleich eine geometrische Voraussetzung, also ein Axiom, dass nämlich diese Figuren auch gleich an Rauminhalt sein sollen ${ }^{1}$. Das wird in XI, 29 benutzt, wo be:wiesen wird, dass Parallelepipeda von derselben Grundfläche und Höhe gleich sind, und ausserdem wird daraus in XI, 28 geschlossen, dass die beiden dreiseitigen Prismen,

1 Cauchy hat bewiesen, dass derartige Figuren wirklich immer kongruent oder symmetrisch sind. 
aus denen ein Parallelepipedon besteht, gleich sind. Nun weiss man, dass die Prismen, die in dem ersten Beweise umgelegt werden, kongruent sind, und dass die dreiseitigen Prismen des letzten Satzes durch Umlegen der Teile in kongruente Prismen verwandelt werden können. Das kann Euklid nicht bemerkt haben; denn dann würde die Einführung eines neuen Princips für die Gleichheit von Körpern überflüssig und deshalb seinem gewöhnlichen Verfahren widerstreitend gewesen sein.

Das 7te Axiom ist in der hier nachgewiesenen Benutzung nur ein Kennzeichen für geometrische Gleichheit gewesen oder, wenn man will, eine Definition davon; jedenfalls aber steckt darin eine wirkliche geometrische Voraussetzung oder ein Axiom von sehr wesentlicher Bedeutung. Dieses drückt aus, dass überhaupt von kongruenten Figuren die Rede sein kann, also von der Verlegung von Figuren an andere Stellen des Raumes. Nach Euklids Axiom bestimmen die geometrischen Grössen alles dasjenige, was während einer solchen Verlegung unverändert bleibt. Worin diese Verlegung bestehen soll, wird jedoch gar nicht charakterisiert, ja sie wird nicht einmal im Axiom erwähnt, aber die Anwendungen zeigen, dass an die empirische Verlegung gedacht wird, die von den physischen, sogenannten unveränderlichen Körpern her bekannt ist.

Wenn wir früher berührt haben, dass das Axiom 1, 7 notwendig ist um eine gerade Linie vollständig $\mathrm{zu}$ charakterisieren, so dachten wir eben daran, dass Euklid, z. B. in den Beweisen für die Kongruenzsätze, bestimmt die Voraussetzung benutzt, dass eine gerade Linie sich durch Verlegung nicht verändert. 


\section{Anmerkung über die Voraussetzungen der Geometrie.}

Wenn man die zuletztgenannte Eigenschaft der geraden Linie mit denjenigen kombiniert, die bereits in den Postulaten ausgedrückt sind, die Eindeutigkeit der Bestimmung durch zwei Punkte miteinbegriffen, so wird die gerade Linie als solche definiert, die ihrer ganzen Ausdehnung nach mit einer anderen geraden Linie zusammenfällt, wenn sie so verlegt wird, dass zwei ihrer Punkte mit zweien der anderen Geraden zusammenfallen. Dass diese Definition keinen Kreisschluss enthält, obgleich die gerade Linie durch Zusammenlegen mit einer anderen geraden Linie bestimmt wird, erkennt man daraus, dass keine andere Linie diese Eigenschaft besitzt. Dagegen ist das Axiom von der Möglichkeit einer Verlegung vorausgesetzt. Nach der Definition erhält man die gerade Linie als geometrischen Ort für die festen Punkte eines Körpers, der sich dreht, während zwei Punkte fest liegen. Die Konstruktion gerader Linien durch ein Lineal, also durch eine bewegliche gerade Linie, folgt gleichfalls aus der Definition.

Diese Definition findet sich nun allerdings nicht bei Euklid, sondern sie ist eine Zusammenfassung der Eigenschaften, die er faktisch benutzt, und die er nach und nach in Postulaten und Axiomen aufstellt. Diese bestimmen demnächst die Ebene als eine Fläche, die jede Gerade, die durch zwei von ihren Punkten geht, ganz enthält. Diese Bestimmung enthält indessen mehr. als eine gute Definition enthalten darf, da sie die Ebene weder als geome trischen Ort für eine einfach unendliche Anzahl gerader Linien, noch für eine doppelt unendliche Anzahl von Punkten bestimmt. Man kann jedoch die Bestimmung in eine Definition und in ein Axiom oder Postulat zerlegen. Die Ebene muss dann zuerst definiert werden als der Ort für die Geraden, die einen festen Punkt mit den Punkten einer festen Geraden verbinden, und demnächst muss als unbeweisbare, aber für das geometrische Lehrgebäude notwendige Voraussetzung hinzugefügt werden, dass diese Fläche dann die oben erwähnte allgemeine Eigenschaft hat.

Über diese Schwierigkeit kommt man dadurch nicht hinweg, dass man, wie es auch wohl geschehen ist, die Ehene definiert als geometrischen Ort für die Punkte, die gleichen Abstand von zwei festen Punkten haben. Es gelingt dann wohl in der Stereometrie 
zu beweisen, dass die so definierte Ebene in der That jede Gerade enthält, von der sie zwei Punkte enthält, aber es gelingt das nur auf der Grundlage der ebenen Geometrie, in der man bereits diese Voraussetzung über die Ebene, die alle behandelten Figuren enthält, gemacht hat.

Mit Bezug auf die Ebene wird noch eine Voraussetzung gemacht, die sich nicht aus der hier aufgestellten Definition ableiten lässt, nämlich die, welche im 5ten Postulat enthalten ist, dass abgesehen von einem genauer bezeichneten Fall - zwei Geraden derselben Ebene sich schneiden.

Wie wir erwähnt haben, macht Euklid, wenn er es auch nicht so deutlich hervorhebt, noch eine geometrische Voraussetzung, die auch geradlinige Figuren betrifft, nämlich die, dass eine in sich selbst zurücklaufende (gebrochene oder krumme) Linie der Ebene einen endlichen Flächenraum einschliesst, und dass sie von jeder geraden oder in sich selbst zurücklaufenden Linie, die einen ausserhalb und einen innerhalb liegenden Punkt verbindet, in wenigstens zwei Punkten geschnitten wird. Ähnliche Voraussetzungen schliessen sich an die geschlossenen Flächen an; sie spielen aber erst eine Rolle, wenn man weiter geht als Euklid.

Die geometrischen Voraussetzungen, die Euklid benutzt, sind also folgende: 1) das Verlegungsaxiom, 2) und 3) die beiden angeführten Voraussetzungen über eine Ebene, 4) die Voraussetzung (S. 123) über geschlossene Konturen (und Oberflächen). In den wesentlichsten Punkten berührt er diese in seinen Definitionen, Postulaten und Axiomen, die ausserdem Erklärungen der gebrauchten Benennungen, und endlich in den Axiomen 1-3 und 8 klar ausgesprochene Voraussetzungen über die allgemeine Grössenlehre enthalten. Diese letzteren geben nicht nur Worterklärungen, sondern sprechen zugleich die für den Aufbau einer wirklichen Grössenlehre notwendige Voraussetzung aus über Unveränderlichkeit und Veränderlichkeit der Grössen durch Teilung, auf die eine Zusammensetzung aus allen oder aus einigen Teilen folgt.

Die deutliche Weise, in der die wichtigsten geometrischen Voraussetzungen also bei Euklid hervortreten, hat die in seinen Elementen aufgestellte Grundlage der Geometrie zu einem guten Ausgangspunkt gemacht für die Untersuchungen der neueren Zeit über den Bereich der einzelnen Voraussetzungen und über ihre gegenseitige Unabhängigkeit. Insofern sie wirklich unter einander unabhängig sind, muss man nämlich einige von 
ihnen festhalten und aus ihnen allein Konsequenzen ziehen können, während man andere unbeachtet lässt. Dadurch erhält man eine Verallgemeinerung der Geometrie; denn die Sätze, welche man dann beweist, gelten sowohl für den "Raum», der zugleich den übrigen Voraussetzungen genügt, als auch für solche Räume, die es nicht thun. Sie können auch in dem durch Euklids Voraussetzungen definierten Raum eine derartige weitergehende Anwendung finden, dass beispielsweise gerade Linien mit Kurven vertauscht werden, die durch einige Eigenschaften der gewöhnlichen geraden Linie charakterisiert werden, so dass ausser dieser auch andere Linien darin einbegriffen sind.

Die wichtigste dieser Verallgemeinerungen, die projekti. vische Geometrie, ist jedoch nicht eigentlich durch Spekulationen über die Axiome entstanden. Ihre meisten Sätze sind aus Verallgemeinerungen hervorgegangen, die sich innerhalb der auf allen Voraussetzungen Euklids aufgebauten Geometrie als zweckmässig erwiesen haben. In Wirklichkeit setzt sie sich jedoch über einige von diesen Voraussetzungen hinweg, und deshalb kann sie auch von Anfang an ohne diese aufgeführt werden. Dasjenige, von dem man in der projektivischen Geometrie absieht, ist das Verlegungsaxiom und die darauf gegründete Lehre von geometrischen Grössen, und dadurch kommt man auch nicht dazu - wenigstens so lange die projektivische Geometrie sich nicht selbst einen neuen Begriff von Verlegung und dadurch einen neuen Grössenbegriff bildet - die durch Euklids übrigen Axiome festgelegten allgemeinen Grössenbegriffe $\mathrm{zu}$ benutzen. Berücksichtigt werden dagegen die in den Postulaten enthaltenen Voraussetzungen, wohei jedoch von den Entlehnungen abzusehen ist, die auch darin aus der Grössenlehre gemacht sind. Dadurch fällt einmal das dritte Postulat über die Bestimmung des Kreises ganz fort, da der Kreis im voraus dadurch definiert ist, dass sein Radius eine unveränderliche Grösse haben soll, und zweitens fallen die Einschränkungen beim 5ten Postulat fort, so dass dieses folgenden Wortlaut enthält: zwei Geraden derselben Ebene schneiden sich immer in einem Punkte. Den direkten Widerspruch mit derjenigen Geometrie, in der alle euklidischen Voraussetzungen benutzt werden, oder mit "der euklidischen Geometrie» vermeidet man dadurch, dass man den parallelen Geraden der letzteren unendlich ferne Schnittpunkte beilegt. Dadurch dass sie überhaupt nicht fragt, in wieweit Schnittpunkte unendlich fern sind oder nicht, ge- 
15. Anmerkung über die Voraussetzungen der Geometrie. 135

langt die projektivische Geometrie dahin, sowohl die euklidische als auch die im Folgenden erwähnte nicht-euklidische Geometrie in sich $\mathrm{zu}$ begreifen.

Die gerade Linie erhält in der projektivischen Geometrie dieselben Eigenschaften wie in der euklidischen mit Ausnahme der Verlegung, durch die eine gerade Linie zum Zusammenfallen mit einer anderen gebracht wird, und die Eigenschaften der Ebene werden ebenso wie bei Euklid an diejenigen der Geraden angeschlossen. Die beiden Voraussetzungen, die sich an die Bestimmung der Ebene knüpften, werden beibehalten. Da man nun nur auf diesen und nicht mehr auf dem Verlegungsaxiom weiter bauen darf, so ist ersichtlich, dass die projektivische Geometrie, wenn sie selbständig entwickelt wird und man nicht die euklidische Geometrie als im voraus bekannt betrachtet, erst einen wirklichen Inhalt bekommt, wenn man aus einer einzelnen Ebene herausgeht und dadurch dahin gelangen kann diese Voraussetzungen zu benutzen. Was die euklidische Voraussetzung über geschlossene Kurven betrifft, so zeigt es sich nämlich, dass diese nicht in der Weise von den fortgefallenen Voraussetzungen unabhängıg ist, dass sie in der projektivischen Geometrie beibehalten werden kann; im Gegenteil erhält man in dieser zwei Arten geschlossener Linien in der Ebene, von denen die eine eine Gerade in einer geraden Anzahl von Punkten (oder in keinem) schneidet, die andere in einer ungeraden.

Im Gegensatze zu der projektivischen Geometrie ist die sogenannte nicht-euklidische Geometrie gerade durch Spekulationen über die von Euklid aufgestellten Voraussetzungen entstanden, namentlich über eine einzelne von ihnen, nämlich diejenige, die wir im 5ten Postulat getroffen haben. Diese Spekulationen versteht man vielleicht am besten, wemn wir daran erinnern, dass dieses Postulat in den meisten Ausgaben seinen Platz unter den Axiomen gefunden hat, wo es durch Einschiebung von anderen weniger echten Axiomen den Namen «Euklids 11tes Axiom» erhalten hat. Während die Bestimmung eines Punktes als Schnittpunktes zweier Geraden unter den Postulaten ein natürliches Gegenstück zu der Bestimmung einer Geraden durch zwei Punkte bildete, so musste die daran geknüpfte Begrenzung besondere Aufmerksam. keit erwecken, wenn man dieselbe Voraussetzung unter den den Lehrsätzen entsprechenden Axiomen traf. Das Axiom, dass zwei Geraden, deren innere Winkel auf derselben Seite einer schneidenden dritten eine Summe kleiner als 2 Rechte bilden, sich auf 
dieser Seite schneiden, wurde dann ein Gegenstück zu dem Lehrsatz, dass die Geraden parallel sind, wenn die Summe 2 Rechte beträgt. Diesen letzten Satz beweist Euklid in I, 27 und 16 mit Hülfe der übrigen Voraussetzungen; lässt sich dann das 11te Axiom und der davon abhängige wichtige Satz über die Winkelsumme eines Dreiecks nicht auch beweisen?

Diese Frage hat im Laufe der Zeiten unzählige Versuche zu Beweisen hervorgerufen. Einige von diesen mögen eine gewisse Berechtigung gehabt haben, insofern sie solche andere Voraussetzungen, deren Richtigkeit man ebenso bereitwillig von vornherein einräumen würde, an die Stelie derjenigen von Euklid setzten. Nur hat man nicht immer, so wie Euklid es thut, selbst ausgesprochen und eingeräumt, dass man eine Voraussetzung machte. Wir wollen beispielsweise für das hier erwähnte euklidische Axiom oder für die damit verbundenen Sätze, dass eine gerade Linie eindeutig bestimmt ist, wenn sie durch einen Punkt geht und einer gegebenen Geraden parallel ist, oder dass die Summe der Dreieckswinkel gleich 2 Rechten ist, diejenigen Beweise anführen, die den Weg in gute Lehrbücher aus unserer und der nächst vorhergehenden Zeit gefunden haben. Von diesen verdankt man diejenigen, die wir unter 2 und 3 aufführen, dem französischen Mathematiker Legendre.

1) Man begnügt sich damit das Axiom zu veranschaulichen und so die Leser zu überreden es ebenso anzunehmen, wie sie früher die übrigen geometrischen Voraussetzungen angenommen haben.

2) Man kann den Satz, dass zwei Winkel eines Dreiecks den. dritten bestimmen, an die Bestimmung eines Dreiecks aus einer Seite und den beiden anliegenden Winkeln anschliessen. Die Grösse einer einzelnen Seite kann nämlich nur den Maassstab für die gezeichnete Figur bestimmen, also keinen Einfluss haben auf die Gestalt des Dreiecks, mithin auch nicht auf die Winkel. Die beiden gegebenen Winkel bestimmen also den dritten. Wie man sieht wird der Beweis dadurch geführt, dass man die Vorstellung vor Ähnlichkeit oder von einer vom Maassstab unabhängigen Gestalt als geometrische Voraussetzung festsetzt, auf der man bauen will. Das, was hier gethan wird, entspricht ganz dem, was Euklid selbst gethan hat, als er im Verlegungsaxiom die Kongruenz als geornetrisches Grössenprincip festsetzte.

3) Andere begnügen sich nicht wie Euklid damit, die Grösse eines Winkels mit Hülfe des Verlegungsprincips $\mathrm{zu}$ bestimmen, 
sondern sie lassen die Grösse eines Winkels das Verhältnis sein zwischen dem von den Schenkeln des Winkels eingeschlossenen unendlichen Flächenstück und der Fläche der ganzen Ebene. Wenn man nun durch einen Punkt zwei Parallelen zu einer gegebenen Geraden ziehen könnte, so würde die eine ganze von der letzten Geraden abgeschnittene Halbebene, die ja gleich zwei Rechten ist, nur einen Teil ausmachen von einem der vier von den beiden ersten Geraden gebildeten Winkel, deren jeder kleiner ist als zwei Rechte. Man sieht leicht, dass die neue Voraussetzung hier in der Winkeldefinition liegt und darauf hinaus läuft, dass das in dieser aufgestellte Verhältnis zwischen zwei unendlichen Grössen einen bestimmten Wert hat.

4) Noch andere beweisen, dass die Summe der Aussenwinkel eines Polygons gleich 4 Rechten ist, indem sie eine Gerade sich allmählich um die Scheitelpunkte der Winkel von der einen anliegenden Seite bis auf die andere drehen lassen. Wenn diese in ihre ursprüngliche Lage zurückkehrt, so soll sie sich im Ganzen um denselben Betrag gedreht haben, wie wenn sie bei der Drehung um einen festen Punkt in ihre ursprüngliche Lage zurückgekehrt wäre. Auch hier ist man nicht bei der in Euklids Verlegungsprincip liegenden Charakterisierung des Winkels als Grösse stehen geblieben, sondern der Winkel ist vielmehr als Teil einer ganzen Umdrehung definiert worden; hierin liegt aber die Voraussetzung, dass dieser Teil eine Grösse von solcher Beschaffenheit hat, dass es gleichgültig bleibt, ob man eine ganze Umdrehung um einen einzelnen Punkt vornimmt oder ob man diese Umdrehung in Umdrehungen um verschiedene Punkte zerlegt. Dass hier wirklich eine Voraussetzung gemacht wird, die ebenso wie Euklids eigenes Axiom speciell für die Ebene gelten soll, das sieht man, wenn man die Ebene mit einer Kugelfläche, die Geraden mit Bogen grösster Kreise vertauscht. Dann hört die Voraussetzung nämlich auf richtig zu sein.

5) Von grösserer Bedeutung mit Bezug auf das Verhältnis von Euklids Axiom zu seinem eigenen Lehrgebäude ist jedoch ein Versuch von Legendre, der sich wirklich an Euklids übrige Voraussetzungen hält. Auf der Grundlage von diesen kann er allerdings nicht den allgemeinen Satz über die Winkelsumme eines Dreiecks beweisen, aber es gelingt ihm doch darzuthun, dass die Winkelsumme nicht grösser ist als zwei Rechte. Einer von den Wegen, auf denen er dies Resultat erreicht, folgt sehr 
genau einem von Euklids eigenen Beweisen, nämlich dem in I, 16. Hier beweist Euklid, dass der Nebenwinkel eines Winkels $C$ in einem Dreieck $A B C$ grösser ist als jeder von den beiden anderen Winkeln, z. B. als $A$. Das wird dargethan, indem man die Mitte $D$ von $A C$ mit $B$ verbindet und darauf $D A_{1}=B D$ abträgt. Das Dreieck $A_{1} B C$ erhält dann dieselbeWinkelsumme wie $A B C$ und enthält einen Winkel $B C A_{1}$, der gleich der Summe der Winkel $A$ und $C$ des ursprünglichen Dreiecks ist; daraus folgt $A+C<2 \mathrm{R}$, oder dass $A$ kleiner ist als der Nebenwinkel von $C$. Die hier angewandte Operation wiederholt Legendre, indem er jedesmal

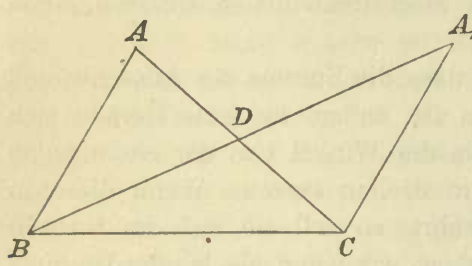
dafür sorgt, dass der Winkel, der hier $B$ genannt ist, der kleinste Winkel des Dreiecks ist, das weiter umgewandelt wird. Das neue Dreieck, zu dem er gelangt, hat dann beständig dieselbe Winkelsumme und enthält einen Winkel, $B$ oder $A$, der gleich oder kleiner ist als die Hälfte des vorhergehenden kleinsten Winkels. Nach einem Princip, das Euklid später in Verbindung mit dem Exhaustionsbeweise aufstellt, kann man auf diesem Wege zuletzt zu einem Dreieck gelangen, in dem ein Winkel kleiner ist als eine beliebige gegebene Grenze. Euklids eigener Satz zeigt, dass die Summe der beiden anderen Winkel kleiner als zwei Rechte ist. Man kann dann leicht, wenn man will mit dem Exhaustionsbeweise, nachweisen, dass die Summe aller drei Winkel, die unverändert dieselbe wie in dem ersten gegebenen Dreieck $A B C$ geblieben ist, nicht grösser als zwei Rechte ist.

War man so weit gekommen ohne das 11te Axiom (das 5te Postulat) zu benutzen, so lag darin die Aufforderung weiter $z u$ gehen. Man musste sich daran halten, dass die Summe der Dreieckswinkel gleich 2 Rechten oder kleiner sein konnte. Wenn das letztere der Fall war, so konnte man beweisen, dass die Winkelsumme abnahm, wenn die Fläche des Dreiecks zunahm. Als Ausgangspunkt für die Untersuchung darüber, ob gerade Linien sich schneiden, hatte man nun nur den, den wir die Voraussetzung über geschlossene Konturen genannt haben, aber man gelangte doch dahin zu beweisen, dass das Schneiden zwischen einer gegebenen Geraden und einer Geraden durch einen gegebenen Punkt stattfindet, wenn diese Gerade in das eine Paar Scheitelwinkel fällt, die von zwei 
15. Anmerkung über die Voraussetzungen der Geometrie. 139

bestimmten, durch den gegebenen Punkt gehenden, geraden Linien gebildet werden. Andere Sätze dieser sogenannten nicht-euklidischen Geometrie, die von Lobatschewsky und Bolyai entwickelt ist, stimmen mehr mit denen der gewöhnlichen euklidischen Geometrie überein.

Noch können wir eine Art von Geometrie anführen, die von Euklids Voraussetzungen ausschliesslich diejenige über geschlossene Konturen und die entsprechenden räumlichen Voraussetzungen benutzt; diese nennt man Analysis situs.

Mit den hier angedeuteten geometrischen Untersuchungen über die Voraussetzungen der Geometrie hat man in unseren Tagen auch erkenntnistheoretische Fragen danach, woher wir sie haben, verbunden. Sind die Voraussetzungen vollkommen willkürlich? oder sind sie auf angeborenen Vorstellungen aufgebaut? oder enthalten sie Wahrheiten, die man durch Erfahrung kennen gelernt hat? Im letzteren Falle darf man nicht sagen, dass die in den Voraussetzungen enthaltenen Behauptungen absolut richtig sind, sondern nur, dass die Abweichungen zu klein gewesen sind um wahrgenommen $\mathrm{zu}$ werden. Auf solche Fragen giebt Euklid, nach dem von uns bereits Gesagten, durchaus keine Antwort. Ihm genügt es, Voraussetzungen aufzustellen, und zu beweisen, dass, wenn sie gültig sind, alles, was daraus abgeleitet wird, es auch ist. Es wird dann Sache desjenigen, der seine Resultate benutzen will, mit sich darüber ins Reine zu kommen, wie weit er die Voraussetzungen anerkennen will.

\section{Die allgemeine Lehre von den Proportionen; Euklids 5tes und 6tes Buch.}

Wir haben im Vorhergehenden verschiedentlich Gelegenheit gehabt auf diejenigen Stellen in Euklids ersten Büchern aufmerksam zu machen, in denen die Behandlung von der jetzt gebräuchlichen abweicht. Diese Abweichungen beruhten, sowieit sie nicht von rein formeller Natur waren, im wesentlichen darauf, dass Euklid sich in diesen ersten Büchern den Gebrauch der Proportionen versagen muss und deshalb Beweise liefert, die auf der 
geometrischen Algebra aufgebaut sind. Der Grund hierfür war wie schon erwähnt der, dass man längst eingesehen hatte, dass die ältere Lehre von den Proportionen streng genommen nur auf kommensurable Grössen anwendbar war. Diesem Übelstande hatte Eudoxus durch eine neue und wahrhaft allgemeine Lehre von den Proportionen abgeholfen, aber an die Entwickelung dieser macht Euklid sich erst im 5ten Buche. Bei diesem Buche wollen wir ausführlicher verweilen um die Proportionslehre genauer kennen zu lernen, die nicht nur ein Hauptfundament für die nachfolgende antike Mathematik wurde, sondern zugleich die Grundlage für die allgemeine Grössenlehre kommender Zeiten enthält.

Die grosse sachliche Bedeutung dieses Buches werden wir am besten ans Licht ziehen können, wenn wir von Euklids vielen Benennungen für Proportionen, die auf verschiedene Weise aus anderen gebildet werden, absehen und diese Bildungen in der modernen algebraischen Zeichensprache wiedergeben. $\mathrm{Zu}$ dem Zweck wollen wir mit den ersten Buchstaben $a, b, c \ldots$ des Alphabetes allgemeine Grössen bezeichnen, die Euklid durch Strecken darstellt, und durch $m, n, p \ldots$ ganze Zahlen, denen Euklid in den Figuren je nach den Beispielen passende kleine Werte erteilt. Aus seinem Buche wird hervorgehen, dass auch diese geometrische Darstellung einen recht guten Überblick gewährt. Von den zahlreichen Definitionen haben wir dann nur Verwendung für die folgenden drei.

In Def. 4 wird gesagt, dass zwei Grössen ein Verhältnis bilden, wenn Vielfache jeder einzelnen von ihnen dahin gebracht werden können die andere zu übertreffen. Hiermit wird nicht nur gesagt, dass die Grössen von derselben Art sein sollen, so dass sie überhaupt verglichen werden können, sondern es wird zugleich eine weiter- 
gehende Forderung ausgesprochen, die sich als unentbehrlich erweisen wird sowohl bei der Ausdehnung der Proportionslehre auf inkommensurable Grössen, als späterhin bei den infinitesimalen Untersuchungen, die sich bei Euklid und Archimedes mittels des auch von Eudoxus erfundenen Exhaustionsbeweises ausgeführt finden.

In Def. 5 wird gesagt, dass

$$
a: b=c: d,
$$

wenn $m a \gtreqless n b$ mit sich bringt, dass $m c \gtreqless n d$, und in Def. 7, dass

$$
a: b>c: d,
$$

wenn es solche Werte von $m$ und $n$ giebt, dass

$$
m a>n b \text {, aber } m c \leqq n d .
$$

Allerdings wird in 5 nicht das Wort "gleich» für die beiden Verhältnisse gebraucht; da aber später in den Sätzen 11 und 13 bewiesen wird, dass $a: b=c: d$ und $c: d \geqq e: f$ es mit sich bringen, dass $a: b \geqq e: f$, so ist eben von Gleichheit die Rede. Die Bedeutung dieser Definitionen von der Grösse eines Verhältnisses wird klar, wenn man beachtet, dass sie in Wirklichkeit identisch sind mit der modernen Bestimmung einer irrationalen reinen Zahl durch rationale Näherungswerte. Erstens ist nämlich eine reine Zahl das Verhältnis einer Grösse zu einer Einheit derselben Art. Zweitens laufen Euklids Vergleiche eben hinaus auf Vergleiche von Verhältnissen mit rationalen Näherungswerten $\frac{n}{m}$.

Nun wollen wir sehen, wie diese Definitionen einer exakten Lehre von Verhältnissen und Proportionen zu Grunde gelegt werden. 
In den Sätzen 1-3 und 5-6 werden folgende Hülfssätze vorausgeschickt:

$$
\begin{array}{rlrl}
m a \pm m b & =m(a \pm b), & & (1 \text { und } 5) \\
m a \pm n a & =(m \pm n) a, & & (2 \text { und } 6) \\
n \cdot m a & =n m \cdot a . &
\end{array}
$$

Unsere Wiedergabe der letzten drei Sätze ist jedoch insofern etwas frei, als beispielsweise in 2 gesagt wird, dass $m a+n a$ dasselbe Vielfache von $a$ ist wie $m b+n b$ von $b$; aber sowohl die Beweise - durch Zerlegung der ganzen Zahlen in ihre Einer - wie die Anwendungen stimmen durchaus zu unserer Angabe der Bedeutungen.

Diese Hülfssätze und die Definition 4 werden benutzt, um folgende Sätze zu einfachen Folgen der Definitionen 5 und $7 \mathrm{zu}$ machen:

Wenn

so ist

$$
\begin{gathered}
a: b=c: d, \\
m a: n b=m c: n d ;
\end{gathered}
$$

wenn $a \overline{>} b$, so ist $a: c\rangle b: c$, aber $c: a \overline{<} c: b$;

wenn

$$
\begin{aligned}
& a: b=c: d \\
& c: d=e: f, \\
& a: b=e: f,
\end{aligned}
$$

und aus solchen gleichen Verhältnissen lässt sich ein neues ebenso grosses bilden, nämlich

$$
(a+c+e):(b+d+f)
$$

wenn aber

$$
\begin{aligned}
& a: b=c: d \\
& c: d>e: f \\
& a: b>e: f .
\end{aligned}
$$

und

so ist

Als Beispiel für die Beweisführung wollen wir Satz 8 betrachten, in dem, wenn $a>b$, die Bestimmung von zwei solchen ganzen Zahlen $m$ und $n$ verlangt wird, dass 
$m a>n c>m b$. Das wird dadurch erreicht, dass man die Forderung mit den folgenden vertauscht, die nach Definition 4 erfüllbar sind:

$$
\begin{gathered}
m b>c \text { und } m(a-b)>c, \\
\quad(n-1) c<m b<n c,
\end{gathered}
$$

woraus folgt, dass

$$
n c<m a \text {. }
$$

Die Sätze 9 und 10, die die Umkehrungen von 7 und 8 sind, werden antithetisch bewiesen. In 14 wird mit Hülfe der vorhergehenden Sätze bewiesen, dass wenn

$$
\begin{gathered}
a: b=c: d, \\
a \geqq c \text { mit sich bringt, dass } b \geqq d .
\end{gathered}
$$

In 15 wird mit Hülfe von 12 bewiesen, dass

$$
m a: m b=a: b .
$$

Die Sätze 16-19 enthalten folgende Umformungen der Proportion

$$
a: b=c: d \text {. }
$$

Aus dieses erhält man $a: c=b: d$,

$$
\begin{aligned}
(a-b): b & =(c-d): d, \\
(a+b): b & =(c+d): d, \\
a & : b=(a-c):(b-d) .
\end{aligned}
$$

16 und 17 werden mit Hülfe von Definition 5 bewiesen; daneben werden bei Satz 16 die beiden vorhergehenden Sätze benutzt. In Satz 17 wird aus der gegebenen Proportion abgeleitet, dass, für alle Werte von $m$ und $n$, $m a \gtreqless(m+n) b$ mit sich bringt, dass $m c \gtreqless(m+n) d$, woraus folgt, dass

$$
m(a-b) \gtreqless n b \text { mit sich führt, dass } m(c-d) \gtreqless n d .
$$


18 und 19 werden - der erstere antithetisch - aus 16 und 17 abgeleitet.

Eine Umformung vermisst man jedoch, nämlich diejenige in

$$
b: a=d: c .
$$

Da diese ausdrücklich im Beweise für 20 angewandt wird, so hat man sie in einem Zusatz zu Satz 7 suchen wollen, was jedoch misslich ist, da in 7 nur die Rede von dem Fall ist, wo $b=d$. Deshalb haben einige Herausgeber den Zusatz nach Satz 4 verlegen wollen. Die Sache ist von keiner grossen Bedeutung, da die Umformung eine unmittelbare Folge von Definition 5 ist.

Die Sätze 20-23 enthalten die wichtige Lehre von zusammengesetzen Verhältnissen. 22 sagt aus:

wenn

so folgt, dass

$$
\begin{gathered}
a: b=d: e \text { und } b: c=e: f, \\
a: c=d: f .
\end{gathered}
$$

Als Vorbereitung auf den Beweis wird in 20 bewiesen, dass nach den Voraussetzungen die Bedingung $a \gtreqless c$, woraus nach 8 folgt, dass $d: e=a: b \geqq c: b=f: e$, nach 9 und 10 mit sich bringt, dass $d \geqq f$. Da nun die gegebenen Proportionen sich nach 4 umformen lassen in

und

so muss auch

$$
m a: n b=m d: n e
$$

$$
n b: p c=n e: p f
$$

$m a \gtreqless p c$ mit sich führen, dass $m d \geqq p f$. 
Der Satz 23, der aussagt, dass, wenn

$$
a: b=e: f \text { und } b: c=d: e
$$

auch

$$
a: c=d: f
$$

ist, wird auf dieselbe Weise bewiesen und ist in derselben Weise durch 21 vorbereitet.

In diesen Sätzen wird gesagt, dass das Verhältnis $a: c$ aus den Verhältnissen $a: b$ und $b: c$ zusammengesetzt sei. Fassen wir das antike Verhältnis als eine moderne Zahl auf, so ergiebt sich, dass das aus den beiden Verhältnissen zusammengesetzte Verhältnis dasselbe ist, was man jetzt ein Produkt nennt. Obgleich den Verhältnissen, die zusammengesetzt werden, bestimmte Formen beigelegt werden, da das Hinterglied des einen das Vorderglied des anderen sein soll, so übt das dennoch keine Beschränkung auf. die Zusammensetzung von Verhältnissen aus. Aus der geometrischen Darstellung in VI, 12 ergiebt sich nämlich auch, dass jedes Verhältnis sich so umformen lässt, dass eines von seinen beiden Gliedern einen gegebenen Wert erhält. In VI, 23, wo bewiesen wird, dass das Verhältnis zwischen zwei Parallelogrammen von gleichem Winkel aus den Verhältnissen der Seiten zusammengesetzt ist, sieht man denn auch, dass man den letzteren die Formen $a: b$ und $b: c$ giebt, um sie zusammenzusetzen.

Wenn wir dieses hier schon mit berücksichtigen, so enthalten die Sätze 22 und 23 vollständige Beweise für die Behauptungen, die man jetzt folgendermassen ausdrücken würde: Ein Produkt ist bestimmt durch seine Faktoren, und die Reihenfolge der Faktoren ist gleichgültig.

Die Alten besassen also zwei verschiedene Darstellungen von dem, was man jetzt unabhängig davon, ob die Faktoren rational oder irrational sind, ihr Produkt 
nennt, nämlich die eben geschilderte und die in der geometrischen Algebra benutzte durch Rechtecke. Dass es wirklich im wesentlichen dasselbe ist, was auf diese beiden Arten dargestellt wird, sieht man aus dem eben angeführten 23sten Satze des 6ten Buches. Die Darstellung durch zusammengesetzte Verhältnisse verbindet einen wesentlichen Vorteil mit ihrer grösseren Umständlichkeit. Wäh. rend die geometrische Algebra gewöhnlich nur Produkte aus zwei Faktoren behandelt, die als Rechtecke dargestellt sind, und man sich in den Raum begeben muss, um Produkte aus drei Faktoren als Parallelepipeda dargestellt $\mathrm{zu}$ erhalten, so lässt sich ein Produkt aus einer beliebigen Anzahl von Faktoren als ein aus diesen zusammengesetztes Verhältnis darstellen. Giebt man den Faktoren die Formen

$$
a: b, b: c, c: d, d: e,
$$

so wird das daraus zusammengesetzte Verhältnis $a: e$; dies wird ausdrücklich in Satz 22 ausgesprochen.

Ein Beispiel für den allgemeinen Gebrauch, den die Griechen von zusammengesetzten Verhältnissen machten, haben wir bereits gehabt in der Umwandelung der Aufgabe von der Würfelverdoppelung in die Bestimmung von zwei mittleren Proportionalen. Die fortlaufende Proportion

$$
a: x=x: y=y: b
$$

der Alten drückt also ganz dasselbe aus, was man nun ausdrücken würde durch

$$
\frac{a}{b}=\left(\frac{a}{x}\right)^{3} \text { oder } \frac{b}{a}=\left(\frac{x}{a}\right)^{3} \text {. }
$$

Auf dieselbe Weise werden auch höhere Potenzen ausgedrückt als Verhältnisse zwischen dem ersten und letzten Gliede einer fortlaufenden Proportion, d. h. einer solchen, deren Glieder eine geometrische Reihe (Quotientenreihe) bilden. 
Dass man auch zu Euklids Zeiten in dieser Beziehung weiter war, als sich unmittelbar aus seinem 5ten Buch ergiebt, das sieht man aus IX, 35, wo eine Bestimmung von der Summe der Glieder einer geometrischen Reihe gegeben wird. In unserer Sprache würde die darin enthaltene Untersuchung folgendermassen ausgedrückt werden: Wenn

$$
\frac{a}{b}=\frac{b}{c}=\frac{c}{d}
$$

so ist $\frac{b-a}{a}=\frac{c-b}{b}=\frac{d-c}{c}=\frac{d-a}{a+b+c}$.

Der Satz wird indessen nicht nur ausgesprochen von einer Summe aus drei aufeinander folgenden Gliedern, deren Betrachtung Euklid im Beweise für ausreichend gehalten hat. Da dieser allein auf Sätze des 5ten Buches aufgebaut ist, so ist er allgemeingültig, wenn auch Euklid für den Augenblick ihn nur deshalb mitnimmt, weil er in dem folgenden zahlentheoretischen Satz Verwendung für den Satz hat, dass

$$
1+2+2^{2}+\ldots 2^{n}=2^{n+1}-1
$$

Wir müssen um so viel grösseres Gewicht auf diese Darstellung von Produkten und Potenzen legen, als sie bis in die neuere Zeit hinein die Grundlage geblieben ist für solche algebraische Untersuchungen, die auf Allgemeinheit Anspruch machten und sich nicht auf rationale Zahlen beschränkten.

Der Satz V, 24 sagt aus: wenn

und

$$
a: c=d: f
$$

so folgt, dass $(a+b): c=(d+e): f$;

dieser Satz ist nahe von derselben Art wie diejenigen sind, die den Sätzen über zusammengesetzte Verhältnisse voran- 
gehen; er kann aber erst hier seinen Platz finden, weil der Satz 22 dazu benutzt wird, um aus den beiden gegebenen Proportionen, nach Umkehrung der Verhältnisse in der zweiten (Bildung der reciproken Werte), abzuleiten, dass

$$
a: b=d: e,
$$

wonach sich mit Hülfe von 18 ergiebt, dass

$$
(a+b): b=(d+e): e .
$$

Eine neue Zusammensetzung der Verhältnisse (nach 22) führt dann zu der Proportion, deren Richtigkeit bewiesen werden soll. Die erstgenannte Anwendung von Satz 22 wird dadurch interessant, dass sie zeigt, dass die Division von Verhältnissen keine neuen und besonderen Sätze verlangt.

Der Satz 25 sagt aus, dass, wenn vier Grössen proportional sind, die Summe aus der grössten und kleinsten grösser ist als die Summe der beiden anderen; dieser Satz wird durch 19 bewiesen. Ein besonderer Fall, der hier jedoch nicht erwähnt wird, ist der, dass die Mittelgrösse zwischen zwei Grössen (arithmetisches Mittel) grösser ist als ihre mittlere Proportionale (geometrisches Mittel); dies wird in VI, 27 durch geometrische Algebra bewiesen und liefert den Diorismus für Gleichungen zweiten Grades.

Zwar ist die im 5ten Buche gegebene Lehre von den Proportionen, trotz der geometrischen Veranschaulichung, vollkommen allgemein und auf alle Arten von Grössen anwendbar; aber sie bedurfte einer Ergänzung, die nach der Weise der Alten geometrisch werden musste. Die Existenz der Verhältnisse geht aus den Definitionen hervor, sobald man nur Grössen hat, die nach Definition 4 Verhältnisse bilden können. Indessen ist, wie oben berührt, ein Beweis erforderlich für die Existenz einer sol- 
chen Grösse, die in Verbindung mit einer gegebenen ein Verhältnis von gegebenem Werte bildet, und diese Existenz wird durch geometrische Konstruktion einer vierten Proportionale bewiesen.

Diese geometrische Ergänzung zu der Lehre von den Proportionen findet sich im 6ten Buche, das zugleich die wichtigsten Anwendungen dieser Lehre auf die Geometrie enthält, namentlich auf ähnliche Figuren, sowie ihre Kombination mit der geometrischen Algebra. Das wichtige Ziel, das durch diese Kombination erreicht wird, ist die geometrische Darstellung und Lösung von Gleichungen 2 ten Grades, in denen $x^{2}$ mit einem Koefficienten behaftet ist. Wenn dieser Koefficient a rational war, so verstanden die Alten wohl, wie wir gesehen haben (S. 61), die Gleichung in eine solche mit der Unbekannten $a x$ und ohne Koefficienten des quadratischen Gliedes umzuformen. Wenn der Koefficient dagegen irrational ist und selbst durch eine Strecke dargestellt werden muss, so reicht die gewöhnliche geometrische Algebra mit zwei Dimensionen nicht mehr aus.

In Satz 1, wo bewiesen wird, dass Dreiecke und Parallelogramme von derselben Höhe sich wie die Grundlinien verhalten, findet die euklidische allgemeine Definition von der Gleichheit von Verhältnissen zweckmässige Verwendung. Da gleiche Grundlinien gleiche Flächeninhalte ergeben, so führt eine unmittelbare Anwendung dieser Definition zu dem allgemeinen Satze, ohne dass es nötig wäre, wie es in modernen Lehrbüchern geschieht, den Fall, wo die gleichartigen Grössen kommensurabel sind, nachher zu dem allgemeinen zu erweitern.

Nach diesem Satze folgen in 2 und 3 die Sätze über parallele Transversalen im Dreieck und über die Teilung einer Dreieckssite durch die Halbierungslinie des gegenüberliegenden Winkels. Darauf folgen in $4-7$ die Haupt- 
sätze über ähnliche Dreiecke; diese werden bewiesen durch Konstruktion eines Dreiecks, das dem einen gegebenen ähnlich und dem anderen kongruent wird. Sie werden sofort (in 8) auf ein rechtwinkeliges Dreieck angewandt und auf die beiden Dreiecke, worin dieses durch die Höhe auf die Hypotenuse geteilt wird.

$9-13$ enthalten die Teilung einer Strecke in gleiche oder proportionale Teile, sowie die Konstruktion der dritten Proportionale (d. h. der vierten zu $a, b$ und $b$ ), der vierten und der mittleren Proportionale. Die letzte Konstruktion ist dieselbe, die schon in II, 14 auf Grundlage der geometrischen Algebra angewandt wurde zur Bestimmung der Seite eines Quadrates, das einem gegebenen Rechteck gleich war.

Darauf kommen in 14-23 die Sätze über das Verhältnis zwischen den Flächeninhalten von Figuren. Den Hauptsatz 23 über die Flächeninhalte gleichwinkeliger Parallelogramme haben wir schon besprochen. Im Beweise (19) dafür, dass das Verhältnis ähnlicher Dreiecke - wie wir sagen - gleich dem Quadrate des Verhältnisses zwischen zwei homologen Seiten ist, wird das Verhältnis $a: b$ zwischen diesen beiden, um mit sich selbst zusammengesetzt werden zu können, auf die Form $b: c$ gebracht, so dass das quadratische Verhältnis $a ; c$ wird. Die Satzgruppe enthält auch noch (in 16) den Satz, dass in einer Proportion das Rechteck aus den äusseren Gliedern gleich demjenigen aus den inneren Gliedern ist.

Am Schlusse des Buches werden demnächst in 28 und 29 die durch Hülfe der Proportionslehre verallgemeinerten Flächenanlegungen behandelt. Eine von der Proportionslehre unabhängige Verallgemeinerung besteht darin, dass die Rechtecke mit Parallelogrammen von einem beliebig gegebenen Winkel vertauscht werden; da aber diese letzte Verallgemeinerung ohne Einfluss ist auf die 
geometrisch-algebraische Bedeutung dieser Aufgaben, so wollen wir von ihr absehen und hier nur von Rechtecken sprechen. Die Aufgaben, von denen die Rede ist, sind dann folgende:

An eine gegebene Strecke (a) eine gegebene Fläche $(B)$ als ein solches Rechteck (mit der Höhe $x$ ) anzulegen, dass das fehlende (28) oder überschiessende (29) Rechteck einem gegebenen (mit den Seiten $c$ und $d$ ) ähnlich wird.

Die Auflösungen werden dieselben, die wir aus II, 5 und 6 für die Fälle abgeleitet haben (S. 46-49), wo die fehlenden oder überschiessenden Figuren Quadrate sein sollen, ausgenommen, dass die früheren Quadrate jetzt mit Rechtecken vertauscht werden, die den gegebenen ähnlich sind. Die Ähnlichkeit tritt überdies dadurch

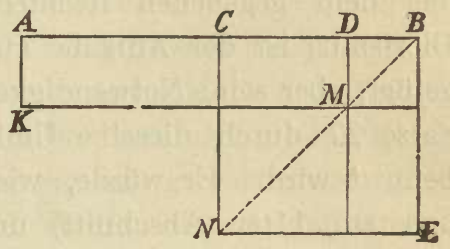
deutlich hervor, dass eine Diagonale der ähnlichen Rechtecke auf eine und dieselbe Gerade fällt. Die erweiterten algebraischen Anwendungen, die sich jetzt erreichen lassen, erkennt man aus der folgenden algebraischen Darstellung der Aufgaben und der Umlegungen von Figuren, wodurch sie gelöst werden, nämlich:

$$
B=a x \mp \frac{c}{d} x^{2}=\mp \frac{d}{c}\left(\frac{a}{2} \mp \frac{c}{d} x\right)^{2} \pm \frac{d}{c}\left(\frac{a}{2}\right)^{2},
$$

wo wir durch die moderne Benutzung der Vorzeichen nur eine zweimalige Darstellung haben vermeiden wollen. Um $x$ zu finden, kommt es darauf an das Rechteck $\frac{d}{c}\left(\frac{a}{2} \mp \frac{c}{d} x\right)^{2}$ zu konstruieren, das dem gegebenen ähnlich, und der Differenz oder Summe der bekannten Flächen 
$\frac{d}{c}\left(\frac{a}{2}\right)^{2}$ und $B$ gleich sein soll. Hierzu dient, wenn $B$ als eine gegebene geradlinige Figur vorausgesetzt wird, die Aufgabe 25, die bereits bei den Pythagoreern erwähnt wurde: Eine Figur zu konstruieren, die einer gegebenen geradlinigen Figur gleich und einer ànderen ähnlich ist.

Die Aufgabe 28 verlangt als Diorismus, dass

$$
B \bar{z} \frac{d}{c}\left(\frac{a}{2}\right)^{2},
$$

oder dass die gegebene Fläche $B$ nicht grösser ist als ein Rechteck über der Hälfte der gegebenen Strecke $a$, das dem gegebenen Rechteck $c d$ ähnlich ist. Dieser Diorismus ist der Aufgabe auf gewöhnliche Weise hinzugefügt, aber seine Notwendigkeit ist in dem vorhergehenden Satze 27 durch dieselbe Umlegung bewiesen, die in 28 benutzt wird. Er würde, wie schon früher bemerkt (in unserem 11ten Abschnitt) unmittelbar aus der Analyse hervorgegangen sein, die der synthetischen Darstellung in 28 entspricht. Wenn das Rechteck $c d$ mit einem Quadrat vertauscht wird, so sagt der Diorismus aus, dass ein Quadrat grösser ist als ein Rechteck von derselben Seitensumme (ein Resultat, das, wie schon bemerkt, auch aus V, 25 folgt).

Satz 30 enthält die stetige Teilung einer Strecke. Die Konstruktion wurde schon einmal in II, 11 (S. 52) angegeben und stützte sich da auf II, 6; nun stützt sich dieselbe Konstrultion auf Satz VI, 29, der eine Verallgemeinerung von II, 6 ist. Der Grund für die Wiederholung ist derselbe wie bei der Konstruktion der mittleren Proportionale, dass nämlich die Aufgabe nun durch die Lehre von den Proportionalen anders ausgedrückt wird als früher (Teilung nach äusserem und mittlerem Verhältnis). 
Satz 31 enthält die Erweiterung des pythagoreischen Lehrsatzes auf beliebige ähnliche Figuren über den Seiten eines rechtwinkeligen Dreiecks. Durch diesen Satz, der von Euklid persönlich herrühren soll, lässt sich die Subtraktion und Addition von Figuren bei den Flächenanlegungen in 28 und 29, wenn $B$ als dem Rechteck $c d$ ähnlich gegeben oder ihm ähnlich gemacht ist, mit Hülfe eines rechtwinkeligen Dreiecks ausführen.

In 33, dem letzten Satze des Buches, wird bewiesen, dass Kreisbogen und die darauf stehendén Centri- und Peripheriewinkel proportional sind.

Wie man sieht, enthalten das 5te und 6te Buch die notwendige Grundlage für eine exakte und vollkommen allgemeine Behandlung solcher Aufgaben, die in unserer Algebra von Gleichungen des ersten und zweiten Grades abhängig sind, durch die Lehre von den Proportionen und durch diese in Verbindung mit geometrischer Algebra. Dass man wirklich diese Grundlage benutzt hat, das ergiebt sich aus den vorliegenden weitergehenden Arbeiten, wie aus der geometrisch-algebraischen Behandlung der Lehre von den Kegelschnitten bei Apollonius und aus einer grossen Menge von den Untersuchungen, die uns Pappus aufbewahrt hat. Dass man sich sogar ausdrücklich für solche algebraische Arbeit rüstete, erkennt man aus verschiedenen Sätzen in Euklids Data, die eine Menge Aufgaben - von der angeführten Art und in den angeführten Formen - nennen, deren Lösung zweckmässigerweise während der fortgesetzten analytischen Arbeit als so bekannt betrachtet wird, dass es genügt, die neuen Aufgaben darauf zurückzuführen.

Was wir als eine Gleichung ersten Grades mit allgemeinen Koefficienten schreiben würden, das wird in den Data der allgemeinen Gültigkeit wegen als eine Proportion ausgedrückt. Um nur ein Beispiel statt vieler zu 
nennen, so sagt Satz 15 der Data aus: Wenn man gegebene Grössen zu jeder von zwei Grössen addiert, die in einem gegebenen Verhältnis stehen, so stehen entweder die Summen selbst in dem gegebenen Verhältnis, oder der Überschuss der einen über eine gegebene Grösse steht in dem gegebenen Verhältnis zu den anderen. Das heisst soviel, dass $x$ bestimmt wird durch die Proportion

$$
(a+m-x):(b+n)=a: b .
$$

Die erste der genannten Alternativen drückt aus, dass $x$ Null werden kann, nämlich wenn

$$
m: n=a: b .
$$

Ein negatives $x$ wird dadurch vermieden, dass man die Gleichung mit der folgenden

$$
(a+m):(b+n-x)=a: b
$$

vertauscht.

Wir haben bereits früher angeführt (S. 107), dass die Data Aufgaben enthalten, die sich unmittelbar auf Flächenanlegungen zurückführen lassen. Als Beispiel für solche Sätze der Data, die Bekanntschaft mit Aufgaben verraten, die mehr indirekt von Gleichungen zweiten Grades abhängen, seien hier 85 und 87 genannt: Wenn zwei Strecken unter einem gegebenen Winkel ein Parallelogramm von gegebener Grösse einschliessen, und die Summe oder Differenz der Quadrate über diesen Strecken gegeben ist, so sind die Strecken auch gegeben. Man kannte mit anderen Worten (unter geometrischer Form) die Lösungen der Gleichungen

$$
x y=a, x^{2} \pm y^{2}=b
$$




\section{Kommensurable Grössen und ihre Behandlung durch Zahlen; Euklids 7,-9. Buch.}

Im 7ten Buche wird eine Einheit eingeführt, wodurch die Grössen, die sie misst, durch ganze Zahlen ausgedrückt werden. In diesem und den beiden folgenden Büchern werden dann ganze Zahlen, sowie ihre Verhältnisse und andere Verbindungen zwischen ihnen behandelt. Im 7 ten Buche begegnen wir für ganze Zahlen solchen Sätzen über Proportionen, die im 5ten Buche bereits auf allgemeingültige Weise bewiesen sind. Zur Erklärung dessen dient der Umstand, dass die allgemeingültige Proportionslehre des 5ten Buches ziemlich neu und deshalb noch nicht genügend entwickelt war, um auf allen den Gebieten, die sie in der Wirklichkeit umfasst, zu Grunde gelegt zu werden. Dadurch ist uns die Proportionslehre des 7 ten Buches überliefert worden als eine Probe von der älteren Behandlungsweise, bei der noch keine Rücksicht auf die Möglichkeit genommen wurde, dass die Glieder der Verhältnisse inkommensurabel sein können.

Dass die Lehre von den Verhältnissen zwischen ganzen Zahlen als einbegriffen in der bereits entwickelten allgemeineren Lehre nicht einfach fortgelassen werden konnte, beruht darauf, dass bei den ganzen Zahlen auch noch andere Rücksichten zu nehmen waren, namentlich auf Fragen nach der Teilbarkeit und auf die Reduktion von Zahlenverhältnissen auf die möglichst kleine Zahl. Das zeigt sich sofort dadurch, dass für Zahlen eine neue Definition der Proportionalität gegeben wird, nämlich in Def. 20. Nach dieser ist $\frac{a}{b}=\frac{c}{d}$, wenn $a$ und $c$ entweder dieselben Vielfachen, oder derselbe aliquote Teil 
oder dieselben aliquoten Teile von $b$ und $d$ sind, $d$. h. wenn gleichzeitig $a=m \cdot \frac{b}{n}$ und $c=m \cdot \frac{d}{n}$. Was die Gleichheit der Verhältnisse betrifft, so enthält diese Definition allerdings nichts anderes, als was schon in der 5ten Definition des 5ten Buches einbegriffen ist; indessen wird man doch bald sehen, dass durch die Art und Weise, wie sie gebraucht wird, eine nicht unwichtige Voraussetzung hineingebracht wird.

In den Sätzen 1-3 werden die bekannten Regeln für die Bestimmung des grössten gemeinschaftlichen Maasses aufgestellt und bewiesen. Direkt wird bewiesen, dass man ein gemeinschaftliches Maass erhält, und antithetisch, dass man das grösste erhält. In 4 wird bewiesen, dass man, wenn $a$ und $b$ ganze Zahlen sind und $f$ ihr grösstes gemeinschaftliches Maass, immer schreiben kann $a=m f$, $b=n f$ und dadurch $a=m \cdot \frac{b}{n}$. Ist $a<b$, so wird $m \geq 1$, $n>1$. Durch diese Bestimmung werden $m$ und $n$ relativ prim. Prüft man nun auf der Grundlage dieser Bestimmung, ob nach Definition $20 \frac{a}{b}=\frac{c}{d}$, so bringt man zugleich die Voraussetzung hinein, dass, wenn es der Fall ist, in $c=m \cdot \frac{d}{n}$ der letzte Faktor $\frac{d}{n}$ eine ganze Zahl ist, dass also $n$, wenn es in einem Produkt $m . d$ aufgeht und relativ prim gegen den einen Faktor $m$ ist, in dem anderen Faktor $d$ aufgehen muss. Dieser Fundamentalsatz der Zahlentheorie ist also bereits in die Voraussetzungen hineingelegt, und es ist folglich nicht von grosser theoretischer Bedeutung, wenn Euklid später auf der Grundlage von diesen mehrere darin einbegriffene Sätze bewreist, wie in 30 den Satz, dass eine Primzahl, die in einem Produkt aufgeht, in einem der Faktoren aufgehen muss. Die er- 
wähnten Voraussetzungen sind namentlich in Satz 20 benutzt, der aussagt, dass, wenn $\frac{a}{b}=\frac{c}{d}$ und $c$ und $d$ so klein wie möglich sind, $c$ in $a$ und $d$ in $b$ aufgeht; dieser Satz bildet ein wichtiges Glied in der Beweisführung, durch die man zu Satz 30 gelangt.

Wie bekannt benutzt man in einem wirklichen Beweise für den genannten Fundamentalsatz den Umstand, dass, wenn $a$ und $b$ relativ prim sind, $k$ der grösste gemeinschaftliche Faktor für $k a$ und $k b$ ist, ein Satz, der aus den Regeln für die Bestimmung des grössten gemeinschaftlichen Faktors folgt, der aber von Euklid nicht mitgenommen wird. - Was man bei Euklid vermisst, das ist ein Beweis dafür, dass die in 4 beschriebene Umformung von $a$ in $m \cdot \frac{b}{n}$ die einzige ist, für die $m$ und $n$ relativ prim sind.

Aus dem Gesagten ergiebt sich allerdings, dass Euklid den Grund für die Lehre von den ganzen Zahlen nicht so tief legt wie den für die Geometrie und für die Lehre von den allgemeinen kontinuierlichen Grössen; die Sorgfalt aber, mit der im übrigen die zahlreiche Reihe von wesentlich theoretischen Sätzen dargestellt und begründet wird, zeugt dennoch sowohl von einem richtigen Blick dafür, dass auch die Arithmetik einer exakten Behandlung bedarf, als auch von einer Benutzung derjenigen Operationen, auf deren Theorie soviel Mühe verwandt wird. Die drei arithmetischen Bücher haben jedoch keine so grosse und grundlegende Bedeutung für die Mathematik bis zu unseren Tagen erhalten wie die vorhergehenden und Teile von den folgenden; deshalb wollen wir uns hier mit ganz wenigen Bemerkungen über ihren ferneren Inhalt begnügen.

Die Lehre von den Verhältnissen im 7 ten Buch wird der Hauptsache nach eine Darstellung der wichtigsten allgemeinen Sätze, die bei der Rechnung mit Brüchen 
benutzt werden. (Deshalb haben wir auch im Gegensatz zu unserer Wiedergabe des 5ten Buches die Verhältnisse als Brüche geschrieben).

Die fortlaufenden Proportionen, die im Sten und 9ten Buche behandelt werden, sind, wie wir bereits erwähnt haben, die antike Form für geometrische Reihen, hier mit ganzen Gliedern. Die Verhältnisse zwischen Gliedern einer solchen Reihe mit verschiedener Stellzahl sind die antike Form für die verschiedenen Potenzen von ganzen Zahlen und Brüchen. Einzelne Sätze über Wurzeln entstehen durch Einschaltung von mittleren Proportionalen.

Die bedeutendsten zahlentheoretischen Sätze, die erreicht werden, sind die Sätze 20 und 36 des 9 ten Buches. Im ersten wird die Unendlichkeit der Reihe der Primzahlen dadurch bewiesen, dass das Produkt der ersten Primzahlen +1 entweder eine höhere Primzahl ist oder eine solche als Faktor enthält. Der zweite sagt aus, dass das Produkt

$$
\left(1+2+2^{2}+\ldots+2^{n}\right) \cdot 2^{n}
$$

wenn der erste Faktor eine Primzahl ist, eine "vollkommene Zahl» wird, d. h. eine solche, die gleich der Summe aller ihrer Faktoren ist. Die Richtigkeit ist leicht dargethan, da - wie bereits früher (S. 147) erwähnt wurde - die dazu notwendige Summation von geometrischen Reihen in Satz 35 dargestellt ist.

\section{Inkommensurable Grössen; Euklids 10tes Buch.}

Wenn wir bei der Besprechung des 10ten Buches, des umfangreichsten der Elemente, auch nicht sehr ins Einzelne gehen, so geschieht das nicht etwa, weil die darin niedergelegte Arbeit, die von Theätet begonnen 
und von Euklid vollendet ist, zu wenig bedeutend sein sollte um unsere volle Aufmerksamkeit $\mathrm{zu}$ verdienen. Im Gegenteil rührt die Schwierigkeit, die sich uns trotz der sorgfältig durchgeführten Bearbeitung entgegenstellt, wenn wir uns einen Überblick über den Inhalt verschaffen wollen, davon her, das es eine beschwerliche Aufgabe ist ohne irgendwelche Zeichensprache zwischen den in diesem Buche klassificierten irrationalen Grössen zu unterscheiden. Dass das Buch dennoch, obgleich man lange Zeit nachher die daraus entnommenen Klassifikationen angewandt finden kann, nicht eine so anhaltende historische Bedeutung hat gewinnen können wie vieles andere bei Euklid, hat seinen Grund darin, dass weiterhin die Zeichensprache, auch schon auf frühen Stufen ihrer Entwickelung, einen viel einfacheren Überblick über die verschiedenen Arten irrationaler Grössen gewährte. Auch wir wollen uns hier damit begnügen mit Hülfe der Zeichensprache Auskunft darüber zu geben, welches die Grössen sind, die klassificiert werden, ohne uns um die Benennungen zu bekümmern, wodurch dies erreicht wird. Was diese angeht, so will ich nur, um direkten Misverständnissen bei Lesern Euklids vorzubeugen, darauf aufmerksam machen, dass er, wenn er von «rationalen» Grössen spricht, damit nicht nur solche meint, die mit der Einheit kommensurabel sind, sondern auch solche, deren Quadrate es sind, die also «nur in der Potenz kommensurabel sind» mit der Einheit. Das Wort Einheit wird hier jedoch nicht so wie in zahlentheoretischen Büchern gebraucht, sondern eine willkürlich gewählte Grösse, die als rational betrachtet wird, spielt in diesem Zusammenhange dieselbe Rolle wie eine Einheit.

Über Kommensurabilität und Inkommensurabilität vergewissert man sich, wie schon früher erwähnt (S. 55), durch direkte Versuche das grösste gemeinschaftliche Maass 
zu bestimmen. Die Inkommensurabilität wird daran erkannt, dass diese Operation sich bis ins Unendliche fortsetzen lässt, während die successiven Reste bis ins Unendliche oder unter jede gegebene Grenze abnehmen. Dieses Abnehmen bis ins Unendliche wird mit derselben wissenschaftlichen Strenge genommen wie jede unendliche Annäherung bei den Alten. Das geschieht auch hier mit Hülfe der 4ten Definition des 5ten Buches, die in Satz 1 dahin umgeformt wird, dass man, indem man von einer gegebenen Grösse mehr als die Hälfte subtrahiert, und von jedem folgenden Reste wiederum mehr als die Hälfte, schliesslich zu einer Grösse gelangen kann, die unter jeder gegebenen Grenze liegt. Mit diesem Satz als Ausgangspunkt werden zuerst einige allgemeine Untersuchungen irrationaler Grössen, ohne Rücksicht auf ihre Entstehung, vorgenommen, und ebenso Untersuchungen von hieraus gebildeten neuen irrationalen Grössen. Dann kommen besondere Untersuchungen über Quadratwurzeln, darunter auch die früher berührten Untersuchungen über diejenigen Fälle, in denen diese sich als rational ergeben, namentlich über rationale rechtwinkelige Dreiecke. Die Formen für irrationale Grössen, die ferner aufgestellt werden, sind vierte $W$ urzeln aus rationalen Grössen und Ausdrücke von der Form $p \pm \sqrt{p^{2}-q}, \sqrt{p^{2}+q} \pm p, \sqrt{a} \pm \sqrt{b}$ sowie die Quadratwurzeln aus diesen Ausdrücken, oder richtiger, wie wir an einem Beispiel sehen werden, gewisse Umformungen dieser Quadratwurzeln in Summen oder Differenzen. Die Glieder der letzteren werden durch Gleichungen von der Formen $x^{2}+y^{2}=a, x \cdot y=b$, wo $a$ und $b$ schon selbst eine gegebene Form haben, bestimmt.

Ausser den Definitionen der verschiedenen Klassen irrationaler Grössen besteht die Arbeit, welche Euklid hier ausgeführt hat, im wesentlichen in Beweisen dafür, dass die gebildeten Grössen irrational und im allgemeinen 
unter einander verschieden sind. Das letztere muss mit einem ausdrücklichen Hervorheben der besonderen Fälle verbunden werden, in denen ein Ausdruck von einer der Formen sich auf eine einfachere Form reducieren oder sich aus Ausdrücken von einfacheren Formen zusammensetzen lässt. Hierher gehört die bekannte Umformung des doppelt irrationalen Ausdrucks $\sqrt{p+\sqrt{p^{2}-q^{2}}}$ in einen einfach irrationalen. Diese Umformung wird in 54 und 91 beziehungsweise für + und - vorgenommen. Dieselbe Transformation wird dann in 57 und 94 angewandt, um den Ausdruck $\sqrt{p \pm \sqrt{p^{2}-q}}$, wo $q$ kein Quadrat ist, umzuwandeln in

$$
\sqrt{\frac{p+\sqrt{q}}{2} \pm \sqrt{\frac{p-\sqrt{q}}{2}}}
$$

Auf diese Form führen die Gleichungen, die in 39 und 76 dazu dienen, die sogenannte "grössere» und «kleinere» irrationale Grösse darzustellen. Die Operationen, wodurch diese und ähnliche Umformungen vorgenommen werden, werden allerdings in der Form der geometrischen Algebra dargestellt, sind aber sachlich dieselben, die man jetzt in der algebraischen Zeichensprache ausdrücken und zur Lösung der entsprechenden Gleichungen anwenden würde.

\section{Elemente der Stereometrie; reguläre Polyeder; Euklids 11tes und 13tes Buch.}

Indem Euklid so im 10ten Buche Gelegenheit findet, eine recht weitgehende algebraische Fertigkeit in der Behandlung solcher Aufgaben zu zeigen, die jetzt durch wiederholte Lösung von Gleichungen zweiten Grades be 
handelt werden, stellt er namentlich die Mittel her um diejenigen Grössen zu benennen, $z u$ denen ihn die Bestimmung der Seiten und Kanten bei regulären Polygonen und Polyedern führt. Ehe er im 13ten Buche hierzu gelangt, muss er zuerst im 11ten Buche die ersten Elemente der Stereometrie darstellen.

In den ersten Sätzen, die über die Lage von Geraden und Ebenen gegen einander handeln, wird man sofort dieselben Sätze und Beweise antreffen, die sich in modernen Lehrbüchern finden. Neben den Sätzen muss Euklid hier wie in der ebenen Geometrie Konstruktionen mitnehmen, da durch diese die notwendigen Beweise für die Existenz der betreffenden Figuren geführt werden. Da Konstruktionen durch Ebenen nicht in der Weise vorbereitet sind wie Konstruktionen durch Geraden es in den Postulaten des ersten Buches waren, so werden die Konstruktionen so weit möglich auf planimetrische zurückgeführt. So wird eine Senkrechte auf eine Ebene von einem Punkte $A$ ausserhalb dieser dadurch in 11 gefällt, dass man zuerst von $A$ eine Senkrechte $A D$ an eine beliebige Gerade $B C$ der Ebene zieht und dann von $A$ eine Senkrechte auf diejenige Gerade der Ebene, die im Fusspunkte $D$ der ersten Senkrechten senkrecht auf $B C$ steht. In 12 erhält man die Senkrechte in einem Punkte einer Ebene dadurch, dass man zuerst von einem ausserhalb liegenden Punkte eine Senkrechte auf die Ebene fällt und dann zu dieser eine Parallele durch den gegebenen Punkt zieht.

Besonders werden solche Sätze mitgenommen, die später Anwendung finden können bei Untersuchungen und Konstruktionen von Parallelepipeden und Polyedern, so in 20 und 21 die bekannten Sätze über die Seiten einer dreiseitigen oder einer beliebigen konvexen Ecke. 
Demnächst wird die Konstruktion einer dreiseitigen Ecke mit gegebenen Seiten in 22 vorbereitet und in 23 ausgeführt. Das geschieht dadurch, dass man auf den Schenkeln dieser Winkel gleiche Stücke abschneidet, dann aus den Seiten, die in den so entstandenen gleichschenkeligen Dreiecken diesen Winkeln gegenüberliegen, ein Dreieck konstruiert und um dieses Dreieck einen Kreis beschreibt; der Mittelpunkt dieses Kreises ist dann die Projektion des Scheitelpunktes der gesuchten Ecke. Die Möglichkeit der Konstruktion, wenn die Seiten den in 20 und 21 gestellten Bedingungen genügen, wird sorgfältig bewiesen und dadurch dargethan, dass diese Bedingungen ausreichend sind.

Im übrigen handelt das Buch namentlich von $\mathrm{Pa}$ rallelepiden und von den Verhältnissen zwischen den Grössen von solchen, und schliesst mit der Bestimmung des Inhaltes eines dreiseitigen Prismas. Die Beweise für die Inhaltsbestimmungen leiden übrigens unter dem Mangel in den geometrischen Voraussetzungen über stereometrische Grössen, den wir früher (S. 130) besprochen haben.

Im 12ten Buche kommt unter anderem die Bestimmung des Inhaltes einer Pyramide vor, über die wir später in Verbindung mit den übrigen Bestimmungen, die im 12ten Buche mittels des Exhaustionsbeweises ausgeführt werden, genauer berichten wollen. Die übrige Fortsetzung der Stereometrie folgt im 13ten Buch, das die Bestimmung der 5 regelmässigen Körper enthält, sowie die Bestimmung der Grösse ihrer Kanten, wenn der Durchmesser der umbeschriebenen Kugel gegeben isł. Hierzu sind zunächst einige geometrisch-algebraische Hülfssätze erforderlich, sowie eine vollständigere Bestimmung der Seiten regulärer Polygone als diejenige ist, die die Konstruktion der Polygone im 4ten Buch unmittelbar giebt. 
Die ersten Sätze lassen sich in der Zeichensprache der jetzigen Algebra folgendermassen ausdrücken: Wenn $x$ und $y$ die Abschnitte der stetig geteilten Strecke $a$ sind, und $x>y$, so ist

1. $x+\frac{a}{2}=\frac{a}{2} \sqrt{5}$ (2 ist die Umkehrung des Satzes 1.)

3. $y+\frac{x}{2}=\frac{x}{2} \sqrt{5}$

4. $a^{2}+y^{2}=3 x^{2}$

5. $a^{2}=x(a+x)$.

Hieraus wird in 6 geschlossen, dass $x$ und $y$ unter die Art von irrationalen Grössen gehören, die im 10ten Buche den Namen "Apotomen» erhalten haben.

Darauf folgen einige Sätze über die Seiten regelmässiger Fünfecke, Sechsecke und Zehnecke. Unter diesen ist in 10 ein eleganter Beweis dafür beachtenswert, dass von den drei genannten Vielecksseiten die erste Hypotenuse, die beiden anderen Katheten eines rechtwinkeligen Dreiecks sind. In 11 wird dann auf Grundlage geometrischer Betrachtungen eine Berechnung der Fünfecksseite vorgenommen, wenn der Durchmesser $d$ des umbeschriebenen Kreises gegeben ist. Euklids eigene Bestimmung führt geradezu dahin, dass die Seite auf die Weise berechnet werden muss, die wir durch den Ausdruck $\frac{d}{2} \sqrt{\frac{5}{2}-\frac{1}{2} \sqrt{5}}$ angeben, aber da Euklid die Mittel fehlen einen solchen Ausdruck aufzustellen, so begnügt er sich damit den Satz auszusprechen, dass die Fünfecksseite, wenn $d$ rational ist, irrational wird von der Form, die er im 10ten Buche «kleinere Irrationale» genannt hat, und seine wirkliche Bestimmung als einen Beweis für diese Behauptung zu gestalten. Dass der Beweis so 
weitläufig wird, beruht darauf, dass ausdrücklich nachgewiesen werden muss, dass die doppelte Irrationalität sich nicht aufheben lässt, denn in solchem Falle würde die irrationale Grösse zu einer anderen Klasse gehören.

In 12 wird die Dreiecksseite bestimmt, und in 13 wird ein reguläres Tetraeder konstruiert und gezeigt, dass die Kante $k$ gleich $d \sqrt{\frac{2}{3}}$ ist, wenn $d$ den Durchmesser der umbeschriebenen Kugel bedeutet.

In 14 wird ein reguläres Oktaeder konstruiert und nachgewiesen, dass $k=d \sqrt{\frac{1}{2}}$.

In 15 wird ein reguläres Hexaeder konstruiert und nachgewiesen, dass $k=d \sqrt{\frac{1}{3}}$.

In 16 wird ein reguläres Ikosaeder konstruiert und durch Ausführung der wirklichen Berechnung gezeigt, dass die Kante eine «kleinere Irrationale» ist.

In 17 wird ein reguläres Dodekaeder konstruiert und durch Ausführung der wirklichen Berechnung bewiesen, dass seine Kante unter die irrationalen Grössen gehört, die Apotomen genannt werden.

18 zeigt an einer und derselben Figur die Konstruktionen der verschiedenen Kanten. Diese Figur wird benutzt um sie unter einander $\mathrm{zu}$ vergleichen.

Die Konstruktionen zeigen, dass die 5 regulären Polyeder wirklich existieren. Im Schlusssatze des Buches wird der Beweis hinzugefügt, dass sie die einzig möglichen sind.

Die meisten Ausgaben von Euklid enthalten noch ein sogenanntes 14 tes Buch, das von dem etwas späteren Mathematiker Hypsikles herrührt, und ein gewiss viel jüngeres 15tes Buch. Man hat sie als Anhänge zum Euklid aufgefasst, weil sie ebenso wie dessen letztes Buch von regulären Polyedern handeln. Das Buch des Hypsikles ist in der That eine weitergehende Behandlung dieses Gegenstandes. Als Beispiel für die darin 
enthaltenen Sätze mag der angeführt werden, dass die um die Seitenflächen eines regulären Ikosaeders und Dodekaeders beschriebenen Kreise gleich gross werden, wenn die beiden Polyeder in dieselbe Kugel beschrieben sind. Als Specialuntersuchung gehört dies Buch eigentlich gar nicht unter die Elemente, aber es ist ein hübsches Beispiel für die Untersuchungen, mit denen sich die Mathematiker der alexandrinischen Zeit beschäftigten. Nach der Vorrede bildet es die Fortsetzung ähnlicher Untersuchungen, die von dem grossen Geometer Apollonius herrühren.

In diesem Zusammephange wollen wir auch noch eine andere Arbeit nennen, die sich an die Untersuchung regulärer Polyeder angeschlossen hat, nämlieh Archimedes' Bestimmung von halbregulären Polyedern, d. h. von solchen, die von regulären Polygonen verschiedener Art begrenzt werden. In einer Arbeit, die verloren gegangen ist, von der uns aber Pappus berichtet, fand Archimedes, dass es 13 solcher Körper giebt.

\section{Der Exhaustionsbeweis; Euklids 12tes Buch.}

Wesentlich dieselben Mittel, durch welche er in der Lehre von den Proportionen eine exakte Behandlung auch von solchen Grössen erreichte, die sich nur näherungsweise durch rationale Zahlenverhältnisse bestimmen lassen, brachte Eudoxus auch in Anwendung für die exakte Bestimmung solcher Grössen, die als Grenzwerte für eine unendliche Annäherung entstehen, und für das Operieren mit ihnen. Die Methode, die er erfand um diese Grenzwerte sicher zu stellen ohne direkten Gebrauch von dem, damals von den Mathematikern in den Bann gethanen, Unendlichkeitsbegriffe $\mathrm{zu}$ machen, hat so bestimmte For- 
men, dass sie ihren eigenen Namen verdient. Wir wollen den Namen gebrauchen, den man ihr im 17ten Jahrhundert gegeben hat, und sie den Exhaustionsbeweis nennen. Dieser wird auf der Voraussetzung aufgebaut, die in der 4ten Definition des 5ten Buches aufgestellt ist, oder gewöhnlich mehr unmittelbar auf dem daraus abgeleiteten Satz 1 des 10ten Buches, dass man, wenn man von einer Grösse die Hälfte oder mehr als die Hälfte wegnimmt und diese Operation eine hinreichende Anzahl von Malen wiederholt, schliesslich zu einer Grösse gelangen kann, die kleiner ist als irgendwelche gegebene Grösse derselben Art. (In der modernen Sprache: $\operatorname{Lim} \alpha \cdot \beta \cdot \gamma \ldots=0$, wenn $\alpha, \beta, \gamma \ldots \overline{\frac{1}{2}}$ ).

Wir wollen den Exhaustionsbeweis kennen lernen aus seiner ersten Anwendung bei Euklid, wo er in XII, 2 benutzt wird um zu beweisen, dass die Flächen zweier Kreise sich wie die Quadrate ihrer Durchmesser verhalten. Im vorhergehenden Satz 1 ist bewiesen, dass ähnliche einbeschriebene. Polygone sich wie die Quadrate ihrer Durchmesser verhalten. Man kann dann, wenn man sich kurz ausdrücken will, sagen, dass der Beweis für 2 darin besteht, die Kreise als Grenzen für solche Polygone zu betrachten. Die Zuverlässigkeit dieses Grenzüberganges wird durch den Exhaustionsbeweis gewährleistet. Die hierzu dienende Anwendung von X, 1, welche erst im Beweise selbst vorkommt, läuft in diesem Falle darauf hinaus, dass man in einen Kreis ein Polygon mit so vielen Seiten beschreiben kann, dass die Differenz zwischen diesem und dem Kreise kleiner wird als irgendwelche gegebene Grenze. Die Dreiecke, die man bei einer Verdoppelung der Seitenzahl von den Segmenten abzieht, aus denen diese Differenz besteht, sind nämlich halb so 
gross wie Rechtecke, die diese Segmente ganz enthalten, also selbst grösser als die Hälfte der Segmente.

Um nun, wenn $A$ und $B$ die Kreise, $a$ und $b$ ihre Radien sind, zu beweisen, dass

$$
A: B=a^{2}: b^{2},
$$

nimmt man an, dass

$$
a^{2}: b^{2}=A: C .
$$

Um zu prüfen, ob es dann möglich ist, dass $C<B$, beschreibt man in $A$ und $B$ ähnliche, reguläre Polygone $A^{\prime}$ und $B^{\prime}$ mit so vielen Seiten, dass $B-B^{\prime}<B-C$, also $B^{\prime}>C$. Dann müsste man haben

$$
a^{2}: b^{2}=A: C=A^{\prime}: B^{\prime}
$$

das ist aber unmöglich, da $A>A^{\prime}$, aber $C<B^{\prime}$.

Der Fall, wo $C>B$, wird auf den vorhergehenden zurückgeführt, da man aus $C>B$ würde ableiten können, dass

wo

$$
\begin{gathered}
b^{2}: a^{2}=C: A=B: D, \\
D<A .
\end{gathered}
$$

Es ist klar, dass derselbe Beweis immer, wenn variierende Grössen $A^{\prime}$ und $B^{\prime}$ die Grenzwerte $A$ und $B$ haben, und das Verhältnis $A^{\prime}: B^{\prime}$ einen konstanten Wert hat, benutzt werden kann um darzuthun, dass das Verhältnis $A: B$ denselben Wert hat. Wenn im besonderen $A^{\prime}=B^{\prime}$, so giebt das $A=B$. Die Alten stellen jedoch nicht, so wie man es in der modernen Lehre vom Unendlichen thun würde, diesen Satz ein für allemal auf, denn das würde ebensoviel sein, als wenn sie solche Begriffe wie unendliche Annäherung erklären und dadurch anerkennen wollten; vielmehr wiederholen sowohl Euklid wie später Archimedes dieselben Beweisformen jedes einzelne Mal, wo sich Verwendung dafür findet. 
Bereits in Satz 5, in dem bewiesen wird, dass zwei dreiseitige Pyramiden von derselben Höhe sich wie die Grundflächen verhalten, hat Euklid Gelegenheit die angeführte Beweisführung zu wiederholen, nachdem er in 3 und 4 bewiesen hat, dass die Voraussetzungen für ihre Anwendbarkeit vorhanden sind. Das geschieht dadurch, dass eine dreiseitige Pyramide, wie in nebenstehender Figur, durch die Ebenen $E F G, E G I H$ und $E H K$, die

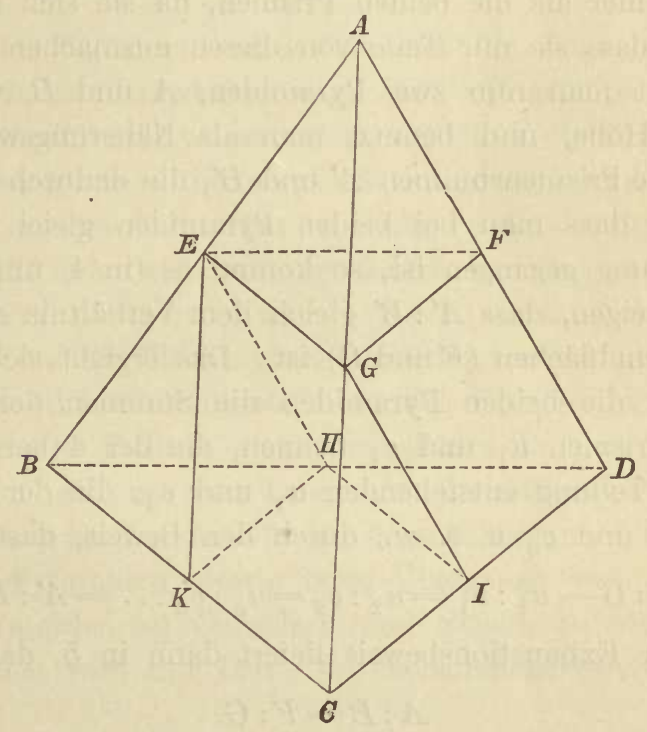

durch die Mitten von 3 oder 4 Kanten gelegt sind, zerlegt wird in zwei ihr ähnliche Pyramiden von halb so grossen linearen Dimensionen, und in zwei Prismen, von denen sich mit Hülfe der Sätze des vorhergehenden Buches zeigen lässt, dass sie gleich gross sind, und von denen das eine dieselbe Höhe und Grundfläche hat wie eine der kleinen Pyramiden. Teilt man nun wieder jede von den kleinen Pyramiden auf dieselbe Weise, und fährt 
man damit fort, so wird man auf diese Weise als Näherungswerte für die Pyramide die Summe der 2 ersten, der 4 folgenden, der 8 folgenden u. s. w. Prismen erhalten. Man sieht, dass die Prismen, die man jedesmal, wenn man zu einer neuen Annäherung übergeht, von der Pyramide fortnimmt, mehr als die Hälfte von dem betragen, was übrig bleibt; die beiden kleinen Pyramiden, die bei der Teilung einer Pyramide entstehen, sind nämlich kleiner als die beiden Prismen, da sie sich so legen lassen, dass sie nur Teile von diesen ausmachen.

Hat man nun zwei Pyramiden, $A$ und $B$, von derselben Höhe, und benutzt man als Näherungswerte für diese die Prismensummen $A^{\prime}$ und $B^{\prime}$, die dadurch gebildet werden, dass man bei beiden Pyramiden gleich weit in der Teilung gegangen ist, so kommt es (in 4) nur darauf an zu zeigen, dass $A^{\prime}: B^{\prime}$ gleich dem Verhältnis zwischen den Grundflächen ( $F$ und $G$ ) ist. Das ergiebt sich, wenn wir für die beiden Pyramiden die Summen der beiden ersten Prismen $u_{1}$ und $v_{1}$ nennen, die der 4 bei der folgenden Teilung entstehenden $u_{2}$ und $v_{2}$, die der 8 nächsten $u_{3}$ und $v_{3}$ u. s. w., durch den Beweis, dass

$$
F: G=u_{1}: v_{1}=u_{2}: v_{2}=u_{3}: v_{3} \ldots=A^{\prime}: B^{\prime} \text {. }
$$

Der Exhaustionsbeweis liefert dann in 5, dass

$$
A: B=F: G \text {. }
$$

Die Bedeutung des hier benutzten Verfahrens erkennt man am besten, wenn man beachtet, dass der Satz 3 die Bedingungen liefert, die nach $\mathrm{X}, 1$ gewährleisten, dass

$$
A=u_{1}+u_{2}+u_{3}+u \text {. s. w. bis ins Unendliche. }
$$

Diese Betrachtung ruft den Wunsch hervor, die konvergente Reihe genauer zu untersuchen. Man sieht nun leicht, und teilweise wird das auch von Euklid in XII, 4 benutzt, dass jedes von den beiden gleich grossen Prismen 
in $u$, zweien von den 4 gleich grossen Prismen in $u_{2}$ ähnlich ist, und so fort, woraus sich ergiebt, dass $u_{2}=\frac{1}{4} u_{1}$, $u_{3}=\frac{1}{4} u_{2}$ u. s. w., oder dass

$$
A=u_{1}\left[1+\frac{1}{4}+\left(\frac{1}{4}\right)^{2}+\ldots\right]=\frac{4}{3} u_{1}=\frac{1}{3} u_{0},
$$

wenn $u_{0}$ ein Prisma bedeutet von derselben Höhe und Grundfläche wie die Pyramide $P$. Für die Richtigkeit dieses Satzes lässt sich leicht ein Exhaustionsbeweis führen.

Dieses Verfahren wendet Euklid allerdings nicht an. Wenn wir es nichtsdestoweniger für wert gehalten haben, es hier anzuführen, wo wir nicht nur die Verfahrungsarten Euklids, sondern überhaupt die der Alten kennen lernen wollen, so geschieht das deshalb, weil Archimedes wirklich, wie wir bald erwähnen werden, ganz dieselbe Summation einer unendlichen Reihe anwendet um die Fläche eines Parabelsegmentes zu finden. Statt dieser Summation wendet Euklid in Satz 7 die bekannte Teilung eines dreiseitigen Prismas in drei Pyramiden an, um den Inhalt der dreiseitigen Pyramide zu finden. Es ist überflüssig, beim Übergange zu mehrseitigen Pyramiden, sowie beim Übergange von Prismen und Pyramiden zu Cylindern und Kegeln zu verweilen; der letztere wird mit Hülfe des Exhaustionsbeweises vollzogen.

Schwieriger ist der in 18 hergestellte Beweis dafür, dass zwei Kugeln sich wie die Kuben der Radien verhalten, denn hier kann man nicht so einfache Näherungswerte bilden wie bei den Kreisflächen. Als Vorbereitung dafür wird die Aufgabe (17) gelöst: in eine Kugel ein Polyeder zu beschreiben, das eine kleinere koncentrische Kugel ganz umschliesst. Diese Aufgabe wird folgendermassen gelöst. In einen grössten Kreis der grösseren Kugel (wir wollen ihn Äquator nennen) wird ein reguläres 
Polygon von gerader Seitenzahl $(2 n)$ so beschrieben, dass es den in derselben Ebene liegenden grössten Kreis der kleineren Kugel ganz umschliesst. Darauf wird in den Äquator der grossen Kugel ein reguläres Polygon von doppelt so vielen Seiten $(4 n)$ beschrieben. Durch dessen Ecken und den Pol des Äquators werden neue grösste Kreise (Meridiane) gelegt, welche vom Schnittpunkt mit dem Äquator an in ebenso viele Teile $(4 n)$ wie der Äquator geteilt werden. Die Teilpunkte werden dann Eckpunkte des gesuchten Polyeders sein, dessen Seitenflächen Trapeze und Dreiecke werden, von denen die Dreiecke um die Pole herum liegen. Aus Euklids vollständigem Beweise geht hervor, dass er diese Lösung hat geben wollen, wenn es auch nicht diese, sondern eine andere unrichtige Lösung ist, die in dem nun vorliegenden Texte dem Beweise vorangeht.

Hier hat man allerdings nicht eine Reihe Näherungswerte für die Kugeln, aber der Exhaustionsbeweis lässt sich dennoch wie früher führen. Sind nämlich $A$ und $B$ die gegebenen Kugeln, $a$ und $b$ ihre Radien, und ist $C$ eine zu $B$ koncentrische Kugel bestimmt durch die Gleichung

$$
A: C=a^{3}: b^{3} \text {, }
$$

so kann man, wenn $C<B$, in $B$ ein Polyeder $B^{\prime}$ beschreiben, das $C$ ganz umschliesst, und in $A$ ein diesem ähnliches Polyeder $A^{\prime}$. Diese werden dann ebenso benutzt wie die Grössen, die wir in dem früher geführten Exhaustionsbeweise $A^{\prime}$ und $B^{\prime}$ genannt haben.

Um zu voller Klarheit über den logischen Wert des Exhaustionsbeweises zu gelangen, wird es nützlich sein, ihn mit modernen Arten der Betrachtung zu vergleichen. Wenn auch die Alten solche Ausdrücke wie Grenzwert einer unendlichen Annäherung durchaus vermeiden, so 
sind es dennoch, wie schon angeführt, in der That solche Werte, die man durch den Exhaustionsbeweis bestimmt. Es ist sogar der exakte Grenzbegriff, der der ganzen Untersuchung zu Grunde gelegt wird, da verlangt wird, dass die (endliche) Annäherung so weit soll gebracht werden können, dass die Abweichung des Näherungswertes vom Grenzwerte kleiner wird als jede gegebene Grösse. Der Exhaustionsbeweis ist ein exakter, antithetischer Beweis für die Eindeutigkeit dieser Bestimmungsart, oder dafür, dass zwei Grössen, die auf diese Weise Grenzen für dieselben Näherungswerte werden, gleich gross sind. Er ist ein notwendiges Glied in jeder vollständigen Infinitesimaluntersuchung, und zudem ist er nichts anderes als ein solches Glied, weshalb man sagen kann, dass da, wo es einen Exhaustionsbeweis giebt, auch eine Infinitesimaluntersuchung vorhanden ist, nämlich die, die zu dem Resultat führt, dessen Richtigkeit hinterher bewiesen wird.

Die Infinitesimaluntersuchungen, die man bei den alten Autoren da findet, wo sie den Exhaustionsbeweis benutzt haben, lassen sich auch auf bestimmte von den infinitesimalen Methoden zurückführen, die man jetzt anwendet. So kann man sagen, dass nicht nur die Pyramiden bei Euklid XII, 5 und das Parabelsegment bei Archimedes, sondern auch die Kreise im Satze XII, 2 durch konvergente Reihen bestimmt sind, und Archimedes benutzt, wie wir sehen werden, solche Summen von unendlich vielen unendlich kleinen Grössen, die wir jetzt bestimmte Integrale nennen. Der Exhaustionsbeweis macht die Anwendung von diesen vollkommen exakt. Die Alten gehen jedoch in den überlieferten Schriften so ganz darin auf diese Exaktheit in jedem einzelnen Falle sicher zu stellen, dass kein Raum dafür bleibt diejenigen Methoden, die sie benutzen um die Resultate zu finden, über den 
augenblicklichen Bedarf hinaus $\mathrm{zu}$ entwickeln und andere neue Methoden zu bilden.

Als im 17ten Jahrhundert die Infinitesimaluntersuchungen namentlich im Anschluss an Archimedes' Schriften wieder aufgenommen wurden, kam es hauptsächlich darauf an, nicht nur seine Begründung der Resultate $\mathrm{zu}$ verstehen, sondern zugleich $\mathrm{zu}$ sehen, wie diese und neue sich finden liessen, also Methoden hierfür zu entwickeln. Man fuhr jedoch fort die nach und nach gewonnenen Resultate zum grossen Teil entweder durch wiederholte Anwendungen des Exhaustionsbeweises sicher zu stellen, oder ihnen doch Zutrauen zu verschaffen durch die Bemerkung, dass dieser Beweis sich würde benutzen lassen. So verfuhr z. B. Fermat, und man fuhr sogar damit fort, nachdem die Differential- und Integralrechnung durch Newton und Leibnitz begründet worden war. Als man sich allmählich mehr in die Benutzung der Methode zur Auffindung neuer Resultate vertieft und sich im Umgehen mit unendlich kleinen Grössen Routine erworben hatte, vergass man jedoch oft die logische Sicherstellung, die der Exhaustionsbeweis $\mathrm{zu}$ geben bestimmt war. Man betrachtete unendlich kleine Grössen als hinreichend definiert durch ihren Namen, und es konnte wohl vorkommen, dass einer sich soweit vergass, eine Grösse als durch eine unendliche Reihe definiert zu betrachten, ohne sich von ihrer Konvergenz überzeugt zu haben.

Erst in unserem Jahrhundert haben die Forderungen nach Exaktheit, denen die Alten durch den Exhaustionsbeweis genügten, wieder volle Anerkennung gefunden. Man erfüllt sie eben dadurch, dass man für die Existenz und Eindeutigkeit der Grenzwerte solche Beweise führt, die im wesentlichen mit dem Exhaustionsbeweise zusammenfallen. Nur führt man diesen jetzt - und dass 
man das kann, deuteten wir bereits bei der ersten Anwendung des Exhaustionsbeweises an - ein für allemal oder wendet ihn doch nur an bei der Behandlung so allgemeiner Begriffe, wie es die Summe einer unendlichen Reihe oder ein bestimmtes Integral sind, während man ihn im Altertum für jede einzelne Anwendung wiederholte.

Eine nicht unwesentliche Formverschiedenheit, die allerdings die Stringenz der Schlüsse unberührt lässt, aber von dem verschiedenen Ausgangspunkt herrührt, ist jedoch vorhanden zwischen' der Behandlung dieser Fragen im Altertum und in der Gegenwart. Sie trat bereits hervor, als im allgemeinen die Rede war von der Kontinuität der Grössen. Das Vorhandensein dieser wurde von den Alten unmittelbar vorausgesetzt durch die geometrisch dargestellten Grössen in Euklids vier ersten Büchern, und erst hinterher lernt man im 5ten Buche arithmetische Mittel kennen, die sich auch benutzen lassen um Grössen zu vergleichen, die nicht kommensurabel sind. Nun dagegen stellt man vielmehr diese arithmetische Grössenbestimmung an die Spitze und wendet sie erst nachher an auf die mehr empirischen, kontinuierlich variierenden Grössen. Ebenso wird man jetzt oft den konvergenten arithmetischen Näherungsprocess, durch den die Fläche einer ebenen Figur, z. B. eines Kreises, oder ein Volumen, z. B. einer Pyramide, bestimmt wird, voranstellen und als Definition für Flächeninhalt oder Volumen benutzen. Die Alten dagegen betrachteten jede ebene Fläche und jedes Volumen als definiert durch die allgemeinen Grössenaxiome, die wir in Euklids erstem Buche kennen gelernt haben. So wurde der Satz, dass der Kreis grösser ist als jedes einbeschriebene und kleiner als jedes umbeschriebene Polygon, eine unmittelbare Folge des 8ten Axiomes. Aus den Axiomen des ersten Buches wurden die Nähe- 
rungsprocesse abgeleitet, die damals für die Bestimmungen angewandt wurden und jetzt für die Definitionen angewandt werden. Die Übereinstimmung besteht darin, dass man damals ebenso wie jetzt sichere Beweise für die Konvergenz dieser Processe verlangte.

Die allgemeinen Grössenaxiome reichen indessen nicht aus, wenn es darauf ankommt die Länge einer krummen Linie oder den Inhalt einer krummen Fläche zu bestimmen. Deshalb hält man es jetzt für besonders notwendig, diese Begriffe durch eben den Näherungsprocess zu definieren, durch den die Grössen faktisch bestimmt werden. Es zeigt sich, dass wenigstens Archimedes für die hier berührte Schwierigkeit ein offenes Auge gehabt hat. Er sucht ihr nicht abzuhelfen durch formelle Definitionen der genannten Begriffe, sondern statt dessen stellt er nach Weise der Alten ausdrücklich die Voraussetzungen auf - postuliert sie -, die er ausser den allgemeinen Voraussetzungen über Grössen in den Näherungsprocessen, wodurch die Grössen bestimmt werden, und in den Beweisen für die Konvergenz dieser Processe anwendet. Diese Voraussetzungen werden als Postulate $\mathrm{zu}$ seiner Schrift über die Kugel und den Cylinder aufgestellt. Sie sagen aus, dass

1) die gerade Linie der kürzeste Weg zwischen zwei Punkten ist;

2) dass von zwei Linien zwischen denselben Punkten, die ihre Konvexität nach derselben Seite wenden, die äussere die grössere ist;

3) dass eine ebene Fläche kleiner ist als eine krumme von demselben Umkreis;

4) dass von zwei krummen Flächen mit demselben (ebenen) Umkreis, die ihre Konvexität nach derselben Seite wenden, die äussere die grössere ist. 
Es ist offenbar ein Missverständnis, wenn man zuweilen in dem ersten von diesen Postulaten - aus seinem Zusammenhang herausgerissen - eine Definition der geraden Linie hat sehen wollen. Vielmehr dient dieses und das folgende zur Definition der Länge einer krummen Linie, die beiden letzten zur Definition des Inhaltes einer krummen Fläche. Dass diese indirekten Definitionen ausreichend sind, zeigt sich dadurch, dass sie sich .wirklich für die Bestimmungen verwerten lassen. Dagegen enthalten 2 und 4, die sicherlich nicht ganz entbehrt werden könnten, etwas mehr als streng notwendig ist.

Nachdem wir nun auch hier die allgemeinen Principien kennen gelernt haben, die Archimedes benutzte um seine infinitesimalen Bestimmungen mittels des Exhaustionsbeweises sicher zu stellen, können wir uns im Folgenden damit begnügen die Zerlegungen, wodurch die Bestimmungen ausgeführt wurden, und die dadurch erreichten Resultate anzugeben, ohne dass es nötig wäre auf die Einzelheiten der strengen Beweisführung einzugehen.

\section{Infinitesimale Bestimmungen bei Archimedes.}

Obgleich die ausserordentlichen Verdienste des Archimedes sich auch auf anderen Gebieten zeigen, die zum Teil schon berührt sind oder später erwähnt werden sollen, so tritt uns seine schöpferische Kraft dennoch vor allem entgegen sowohl in den infinitesimalen Untersuchungen, für die Eudoxus schon eine so zuverlässige Grundlage geschaffen hatte, als auch in der Lehre vom Gleichgewicht, von der man vor Archimedes keine exakte Behandlung kennt. In diesen Untersuchungen hat er vielfach Gelegenheit sich ausser mit der elemen- 
taren Mathematik auch mit der Lehre von den Kegelschnitten vertraut zu zeigen, so vertraut, dass er sogar Schnitte an solchen Flächen behandeln kann, die durch Umdrehung von Kegelschnitten entstanden sind. Da wir jedoch über die griechische Lehre von den Kegelschnitten am liebsten im Zusammenhange reden wollen, so wollen wir in unserer sonstigen Besprechung von Archimedes' Arbeiten nur jedesmal die Eigenschaften der Kegelschnitte erwähnen, die er gerade benutzt, ohne vorläufig zu untersuchen, woher er sie kennt.

Wir wollen unsere Mitteilungen über Archimedes' infinitesimale Untersuchungen mit der. Schrift über die Quadratur der Parabel beginnen, weil wir in dieser Schrift ausnahmsweise erfahren, wie Archimedes von Anfang an zu seinem Resultat gelangt ist, und weil dies Resultat naturgemäss den Anstoss zu den damit verwandten Untersuchungen in anderen Schriften gegeben haben kann.

Archimedes nennt den Weg, auf dem er zuerst die Fläche des Segmentes gefunden hat, das von einem $\mathrm{Pa}$ rabelbogen und seiner Sehne begrenzt wird, mechanisch, weil er sich auf die Sätze von statischen Momenten und vom Schwerpunkt des Dreiecks stützt, die in seinem Buche über das Gleichgewicht ebener Figuren, das später besprochen werden soll, dargestellt werden. Die Untersuchung lässt sich kurz folgendermassen wiedergeben: Nimmt man die Sehne $A C$ (deren Länge wir a nennen wollen) zur Abscissenaxe und den durch den einen Endpunkt $A$ der Sehne gehenden Parabeldurchmesser $A G$ zur Ordinatenaxe, bezeichnen ferner $x$ und $y$ die Koordinaten eines Punktes $E$ der Parabel, $y_{1}$ die der Abscisse $x$ entsprechende Ordinate $Z L$ der Tangente $C G$ im anderen Endpunkt der Sehne, dann ist - was Archinedes zuerst aus den damals bekannten Sätzen über die Parabel ableitet -

$$
a \cdot y=x \cdot y_{1} \text {. }
$$


Die Ordinate $y_{1}$ hat deshalb in der Lage, die sie wirklich einnimmt, dasselbe Moment mit Bezug auf die Linie $A G$, wie die Ordinate $y$ haben würde, wenn man sie parallel

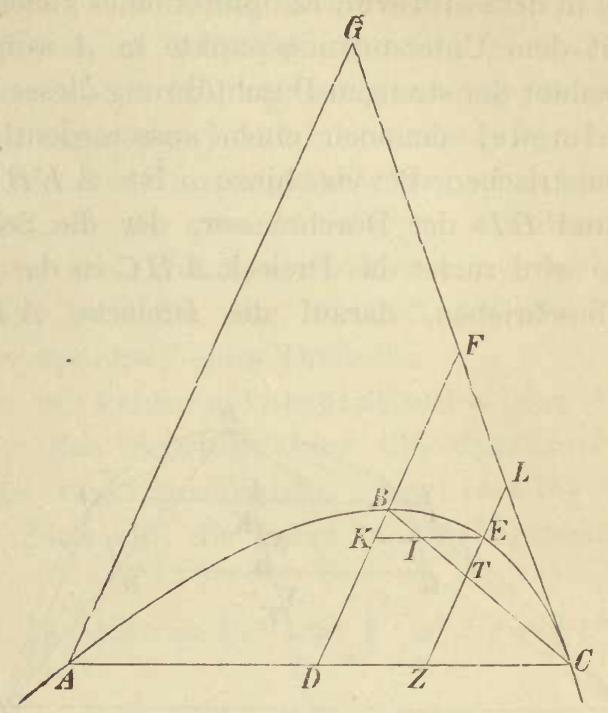

mit sich selbst nach $C$ verlegte. Durch eine Teilung der Figur in Streifen mittels Parallelen zur Ordinatenaxe und durch eine Anwendung des Exhaustionsbeweises, die am ehesten einer vollständigen Begründung des Satzes entsprechen würde, dass

$$
a \int_{0}^{a} y d x=\int_{0}^{a} y_{1} x d x,
$$

beweist Archimedes dann, dass das Moment des in $C$ angebrachten ganzen Parabelsegmentes mit Bezug auf $A G$ dasselbe ist wie das Moment des Dreiecks $A C G$ in seiner natürlichen Lage. Da nun der Abstand des Schwerpunktes des Dreiecks $A C G$ von $A G$ ein Drittel von demjenigen des Punktes $C$ ist, so wird das Segment $\frac{1}{3}$ vom Dreieck 
A C G oder $\frac{2}{3}$ des Dreiecks, das von der Sehne und den 'Tangenten in ihren Endpunkten begrenzt wird. [Bei der genaueren Ausführung hiervon denkt Archimedes sich die Parabel in dem anderen Endpunkt eines gleicharmigen Hebels mit dem Unterstützungspunkte in $A$ aufgehängt].

Ungeachtet der strengen Durchführung dieses Beweises fügt Archimedes dennoch einen ausserordentlich hüb. schen geometrischen Beweis hinzu. Ist $A E B F C$ das Segment und $B D$ der Durchmesser, der die Sehne $A C$ halbiert, so wird zuerst das Dreieck $A B C$ in das gegebene Segment beschrieben, darauf die Dreiecke $A E B$ und

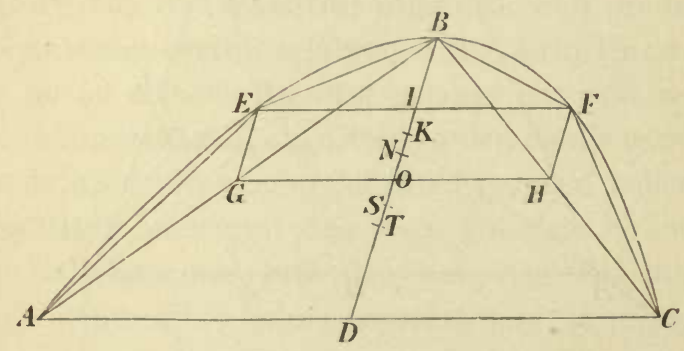

$B F C$ in die abgeschnittenen Segmente, entsprechende Dreiecke in die neuen Segmente u. s. w. Dann ergiebt sich leicht, dass jedes Dreieck (wie $A E B$ ) in einer neuen Reihe von Dreiecken gleich $\frac{1}{8}$ eines Dreiecks (wie $A B C$ ) in der vorhergehenden Reihe ist. Da in jeder neuen Reihe doppelt so viele Dreiecke wie in der vorhergehenden sind, so erhält man

$$
\text { Segment } \begin{aligned}
A B C & =\left[1+\frac{1}{4}+\left(\frac{1}{4}\right)^{2}+\ldots\right] \triangle A B C \\
& =\frac{4}{3} \triangle A B C .
\end{aligned}
$$

Der Beweis wird - wie in unserer Besprechung von Euklids 12tem Buche berïhrt wurde - als ein Exhaustionsbeweis durchgeführt. 
Während Archimedes' geometrische Quadratur der Parabel faktisch auf der Summation einer unendlichen Reihe beruht, haben wir in unserer Wiedergabe der "mechanischen» Bestimmung das Integralzeichen benutzt um eine Zerlegung in Stücke, die sämtlich gleichzeitig bis ins Unendliche abnehmen, zu bezeichnen. Archimedes' Behandlung kann hier jedoch nicht eine Integration genannt werden, sie dient im Gegenteil dazu, eine Integration zu vermeiden, da sie nur die vorliegende Untersuchung auf eine andere zurückführt, deren Resultat vorher schon ohne Integration gefunden war, nämlich auf die Bestimmung des Schwerpunktes eines Dreiecks.

Von wirklichen Integrationen lässt sich dagegen reden in den Schriften über die Spiralen und über Konoide und Sphäroide. Archimedes stellt dort nämlich Sätze auf, die genau unseren Formeln

$$
\int_{0}^{c} x d x=\frac{1}{2} c^{2} \text { und } \int_{0}^{c} x^{2} d x=\frac{1}{3} c^{3}
$$

entsprechen, und wendet diese auf unter sich verschiedene geometrische Bestimmungen an, die man jetzt - mit Ausnahme der bei jeder einzelnen Frage wiederholten Anwendung des Exhaustionsbeweises - auf dieselbe Weise mit Hülfe der angeführten Integralformeln ausführen würde. Diese Sätze, von denen der erste in der Einleitung zu der Schrift über Konoide und Sphäroide angeführt. wird, der zweite in einem Zusatz zu Satz 10 über die Spiralen, sind folgende:

$$
\begin{gathered}
\frac{n^{2}}{2} h<h+2 h+3 h+\ldots+n h<\frac{(n+1)^{2}}{2} h \\
\frac{n^{3}}{3} h^{2}<h^{2}+(2 h)^{2}+(3 h)^{2}+\ldots+(n h)^{2}<\frac{(n+1)^{3}}{3} h^{2} .
\end{gathered}
$$


Der erste ergiebt sich unmittelbar durch eine Summation der Glieder einer arithmetischen Reihe (Differenzreihe), die sicher schon lange bekannt gewesen ist. Der zweite beruht auf einer im Hauptsatz 10 ausgeführten Summation der betreffenden Reihe. Archimedes findet, dass

$$
\begin{gathered}
3\left[h^{2}+(2 h)^{2}+(3 h)^{2}+\ldots+(n h)^{2}\right]= \\
(n+1)(n h)^{2}+h(h+2 h+3 h+\ldots+n h) .
\end{gathered}
$$

Der Beweis, in dem $h, 2 h$ u. s. w. durch Strecken dargestellt werden, lässt sich, wenn wir $h$ als Einheit betrachten und die gesuchte Summe der Quadrate $s$ nennen, folgendermassen wiedergeben:

$$
\begin{gathered}
(n+1) n^{2}=n^{2}+[(n-1)+1]^{2}+[(n-2)+2]^{2}+\ldots \\
+[2+(n-2)]^{2}+[1+(n-1)]^{2}+n^{2} \\
=2 . s+2 .(n-1)+4(n-2)+6 .(n-3)+\ldots \\
+2(n-1) .1 .
\end{gathered}
$$

Addiert man hierzu

$$
(n+n-1+n-2+\ldots+1),
$$

so erhält man

$$
2 s+n+3 \cdot(n-1)+5 \cdot(n-2)+\ldots+(2 n-1) \cdot 1 .
$$

Dass diese Grösse genau gleich $3 s$ ist, geht aus der Summation der folgenden Gleichungen hervor, deren Richtigkeit aus der Formel für die Summe der Glieder einer Differenzreihe folgt:

$$
\begin{aligned}
n^{2} & =n+2(n-1+n-2+\ldots+1) \\
(n-1)^{2} & =n-1+2(n-2+n-3+\ldots+1) \\
(n-2)^{2} & =n-2+2(n-3+n-4+\ldots+1)
\end{aligned}
$$

Diese Summation ist ein wertvolles algebraisches Nebenprodukt ron Archimedes' Untersuchung. 
Die Anwendung, die Archimedes hiervon in seiner Schrift über die Spiralen macht, ist die Berechnung eines Sektors einer archimedischen Spirale $r=a . \vartheta$. Die Fläche eines solchen ist

$$
\frac{1}{2} \cdot \int_{\vartheta_{0}}^{\vartheta_{1}} r^{2} d \vartheta=\frac{1}{2 a} \int_{r_{0}}^{r_{1}} r^{2} d r
$$

wird also durch die letzte der beiden angeführten Integrationen gefunden. Archimedes bestimmt das Verhïltnis $\mathrm{zu}$ einem Kreissektor mit dem Radius $r$, und erreicht dies durch'Teilung des Winkels $\vartheta_{1}-\vartheta_{0}$ und dadurch zugleich der Sektoren, durch Konstruktion von Kreissektoren, die von Spiralsektoren eingeschlossen werden und diese umschliessen, durch Vergleichung mit diesen Kreissektoren, und durch Anwendung des Exhaustionsbeweises.

Als Konoide werden teils Umdrehungsparaboloide bezeichnet, teils Umdrehungshyperboloide mit zwei Netzen, von denen jedoch nur das eine benutzt wird. Sphäroide sind Umdrehungsellipsoide. In der Schrift über diese Arten von Flächen bestimmt Archimedes das Volumen von Segmenten, die von einer solchen Fläche und einer beliebigen Ebene begrenzt werden. Archimedes ist imstande die Beschaffenheit eines willkürlichen ebenen Schnittes an einer solchen Fläshe $\mathrm{zu}$ ermitteln und seine Axen mit Hülfe der Abschnitte zu bestimmen, die seine Ebene auf dem zugehörigen Durchmesser der Fläche abschneidet. Er hat ferner die Fläche einer Ellipse gefunden, was leicht durch Vergleichung von Figuren geschieht, die in die Ellipse und in einen Kreis über ihrer einen Axe als Durchmesser beschrieben werden. Die Volumenbestimlmungen werden dann auf eben die Integrationen zurückgeführt, die Archimedes, wie wir gesehen haben, in einer anderen Form kannte. 
Die beiden angeführten Schriften sind ausser durch die darin enthaltenen Flächen- und Volumenbestimmungen auch noch in anderer Beziehung merkwürdig. So liefert die Schrift über Konoide und Sphäroide, wie sich schon aus dem Angeführten ergiebt, uns Aufklärungen über Archimedes' Bekanntschaft mit den Kegelschnitten. In der Schrift über die Spiralen finden sich einige früher erwähnte «Einschiebungen». Zur Hauptaufgabe dieser Schrift gehört ausser der Flächenbestimmung auch noch eine andere infinitesimale Aufgabe, nämlich die Bestimmung von Tangenten an die Spiralen. Dazu benutzt Archimedes, selbstverständlich unter der gebührenden Kontrole mittels des Exhaustionsbeweises, dasselbe infinitesimale Dreieck, das jetzt für die Bestimmung von Tangenten an Kurven benutzt wird, die in Polar-Koordinaten ausgedrückt sind. Als Resultat ergiebt sich, dass die Polarsubtangente $r . \vartheta$ wird. Die Subtangenten in den Endpunkten der verschiedenen ganzen Umläufe der Spiralen erhielten - wie wir früher (S. 78) berührt haben für Archimedes ein besonderes Interesse dadurch, dass sie geradlinige Darstellungen von Kreisperipherien sind.

Die wichtigste integrationsähnliche Bestimmung, die wir Archimedes verdanken, dürfte jedoch seine Bestimmung der Kugeloberfläche sein. Das Verfahren ist, wenn auch in einer anderen Form, ungefähr dasselbe, das in unseren elementaren Lehrbüchern benutzt wird, und führt, in Übereinstimmung mit dem Titel des Werkes, namentlich dahin, dass Kugelzonen und die entsprechenden Stücke der Mantelfläche des umbeschriebenen Cylinders gleich gross sind. Von da aus gelangt man jedoch leicht $\mathrm{zu}$ anderen Bestimmungsformen und gleichfalls zu der Bestimmung des Volumens von Kugel, Sektor und Segment. Da Archimedes ebensowenig wie Euklid irgend- 
welche Einheit einführt, so bestehen diese letzten Bestimmungen in der Konstruktion von Cylindern und Kegeln, die den gesuchten Raumgrössen gleich sind.

Im zweiten Buch derselben Schrift werden (ausser der eben genannten Bestimmung der Volumina von Segmenten) verschiedene Aufgaben über die gesuchten Raumgrössen behandelt, darunter diejenige, durch eine Ebene eine Kugel in zwei Segmente zu teilen, die in einem gegebenen Verhältnis stehen. Die Lösung hängt wie bekannt von einer Gleichung dritten Grades ab. Auf eine solche führt auch Archimedes die Aufgabe zurück, indem er ihr folgende Form giebt: Eine Strecke $D Z$, auf der die Punkte $B$ und $T$ gegeben sind, so durch einen Punkt $X$ zu teilen, dass

$$
D B^{2}: D X^{2}=X Z: T Z .
$$

$D B$ ist hier der Durchmesser $2 r$ der Kugel, auf dessen Verlängerung $B Z=r$ abgetragen ist, $D X$ ist die Höhe des einen Segmentes, und wenn dieses sich $\mathrm{zu}$ dem anderen verhalten soll wie $m: n$, so ist

$$
T Z=\frac{m}{m+n} \cdot r
$$

Archimedes verspricht diese Gleichung später $\mathrm{zu}$ lösen und hebt für den Augenblick nur hervor, dass der Bedingung für die Möglichkeit, welche die Gleichung verlangt, genügt wird durch die vorliegende Aufgabe über die Kugel. Ein Grund für diesen Aufschub - der leider dazu geführt hat, dass die Auflösung in dem vorliegenden Text fehlt - mag darin gelegen haben, dass Archimedes in demselben Buche mehrfach Verwendung für dieselbe Gleichung hatte. Der letzte (9te) Satz des Buches sagt nämlich aus, dass das grösste von den Kugelsegmenten, die eine gegebene krumme Oberfläche haben, eine Halb- 
kugel ist. Der Beweis, der hierfür geführt wird, der übrigens in dem überlieferten Text nur unvollständig ist, lässt deutlich erkenmen, dass er erst gemacht ist, nachdem das Resultat auf anderem Wege gefunden worden war. Eine wirkliche Ableitung des genannten Satzes hätte dagegen einen natürlichen Platz in demselben Zusatz finden können, den Archimedes mit Bezug auf die Kugelteilung versprochen hatte. Ein solcher Satz über ein Maximum, wie dieser 9te, kommt nämlich stets bei den Griechen als Diorismus zu einer Aufgabe vor. Dieser hat im gegenwärtigen Falle darauf ausgehen müssen ein Kugelsegment $\mathrm{zu}$ finden, dessen Volumen und krumme Oberfläche gegeben waren, und diese Aufgabe lässt sich eben mittels der oben genannten Gleichung lösen.

Nun findet sich wie gesagt der versprochene Zusatz nicht in dem überlieferten Text selbst, aber man nimmt an, dass er in einem anderen alten Manuskript enthalten gewesen ist, das von Eutokius, dem Kommentator des Archimedes, gefunden und im Auszuge wiedergegeben ist. In diesem Manuskript wird die Gleichung des Archimedes mit Hülfe von Kegelschnitten gelöst, und aus der Gleichung werden solche Bestimmungen der Möglichkeit abgeleitet, dass ihre Anwendung auf die Aufgabe, «ein Kugelsegment von gegebenem Volumen und gegebener krummer Oberfläche zu finden» unmittelbar den Satz 9 ergeben würde. Eben diese Lösung soll später mitgeteilt werden, da sie eines der besten überlieferten Beispiele dafür ist, wie die Alten die sogenannten «räumlichen Aufgaben» behandelten.

Dass die Bestimmung der Kugeloberfläche in reichem Maasse Veranlassung $\mathrm{zu}$ weiteren Untersuchungen giebt, hat sich also sofort in Archimedes' Schrift zu erkennen gegeben. Anwendungen in der Praxis oder auf andere Wissenschaften, wie die Geographie, liegen auch nahe. 
Diese Umstände mögen dazu beigetragen haben, dass Archimedes diese Bestimmung unter seinen Arbeiten am höchsten stellte. Ein genügender Grund hierfür lag jedoch schon in dem Umstande, dass es ihm gelungen war, den Inhalt einer nicht abwickelbaren krummen Fläche zu einer Zeit zu berechnen, wo sogar die Berechnung von ebenen Flächen und von Rauminhalten so wenig entwickelt war. Und wie wenige Flächen kennen wir nicht heutigen Tages, deren Inhalte sich auf leidlich übersichtliche Weise darstellen lassen!

Nach Archimedes' Wunsch setzte man auf sein Grab ein Monument, das eine Kugel mit einem umbeschriebenen Cylinder enthielt. Dies fand und erneuerte Cicero anderthalb Jahrhundert später, als er Quästor auf Sicilien war.

\section{Archimedes' Lehre vom Gleichgewicht.}

Die Regeln für das Gleichgewicht eines ungleicharmigen Hebels sind lange vor A rchimedes' Zeit bekannt gewesen, aber bei ihm findet man die erste wirkliche Be-

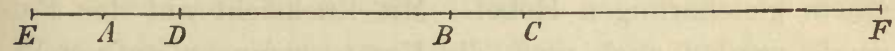

gründung. Wir können seinen Gedankengang kurz folgendermassen wiedergeben. $A$ und $C$ seien die Angriffspunkte der Gewichte $P$ und $Q$, und der Punkt $B$ sei auf $A C$ derartig bestimmt, dass

$$
A B: B C=Q: P \text {. }
$$

Dann ist der Hebel, auf dessen eigenes Gewicht keine Rücksicht genommen wird, im Gleichgewicht, wenn er in 
$B$ unterstützt wird. Der Beweis wird dadurch geführt, dass man den Hebel in einem Punkte $D$ so teilt, dass

$$
A D: D C=P: Q,
$$

und auf seiner Verlängerung $E$ und $F$ so bestimmt, dass $E A=A D$ und $C F=D C$. Man kann dann, ohne die Gleichgewichtsverhältnisse zu ändern, das Gewicht von $P$ gleichmässig über $E D$, und das von $Q$ gleichmässig über $D F$ verteilen, wodurch das ganze Gewicht $P+Q$ gleichmässig über $E F$ verteilt wird. Wegen der Symmetrie findet dann Gleichgewicht statt, wenn $E F$ im Mittelpunkte $B$ unterstützt wird.

Im ersten Buche seiner Schrift über das Gleichgewicht ebener Figuren führt Archimedes einen solchen Beweis ohne jedoch eine kontinuierliche Verteilung $\mathrm{zu}$ benutzen. Er behandelt zuerst den Fall, wo $P$ und $Q$ kommensurabel sind und sich also auf Punkte mit gleichen Abständen verteilen lassen, und geht darauf mittels des Exhaustionsbeweises zu dem Fall über, wo $P$ und $Q$ inkommensurabel sind. Er hat wie gewöhnlich ausdrücklich die Voraussetzungen aufgestellt, auf denen der Beweis aufgebaut wird, und das sind hier die Bedingungen für Gleichgewicht oder für den Mangel an Gleichgewicht bei einem gleicharmigen Hebel. Mit Rücksicht auf das Folgende werden auch noch die Voraussetzungen aufgestellt, dass die Schwerpunkte ähnlicher Figuren homologe Punkte sind, und dass der Schwerpunkt einer Figur, deren Konvexität sich überall nach aussen wendet, auf die Figur selbst fallen muss. Wemn die Beweise dafür, dass der Schwerpunkt eines Dreiecks der Schnittpunkt seiner Medianen ist, mit denen das Buch schliesst, uns jetzt unnötig weitläufig vorkommen, so beruht das darauf, dass sie auf den ausdrücklich aufgestellten Voraussetzungen haben aufgebaut werden müssen. 
Wir haben bereits gesehen, wie Archimedes die Sätze über Gleichgewicht benutzt hat um die Fläche eines Parabelsegmentes zu finden. Später hat er im zweiten Buche der Schrift über das Gleichgewicht ebener Figuren den Schwerpunkt eines Parabelsegments gefunden. Dieser Bestimmung liegt der Satz zu Grunde, dass die Schwerpunkte verschiedener Parabelsegmente ihre Durchmesser nach demselben Verhältnis teilen müssen. Das wird bewiesen durch dieselbe Teilung der Parabelsegmente in unendlich viele Dreiecke, die in seiner "geometrischen» Bestimmung der Fläche des Segmentes ${ }^{1}$ (vergl. die Figur S. 180) benutzt wurde. Der unbekannte Wert des konstanten Verhältnisses lässt sich nun finden durch Zerlegung des Segmentes $A B C$ in das Dreieck $A B C$ und zwei neue Segmente. Die dabei entstehende Gleichung ersten Grades löst Archimedes unter geometrischer Form.

Es ergiebt sich, dass Archimedes noch einen Schwerpunkt kennt, nämlich denjenigen eines beliebigen Segmentes eines Umdrehungsparaboloids. Dieser kann nicht auf dieselbe Art gefunden worden sein wie der Schwerpunkt des Parabelsegmentes, sondern seine Bestimmung muss unter der einen oder anderen Form zurückgeführt worden sein auf die bereits erwähnten, von Archimedes gekannten Integrationen, von denen sie in der That abhängt. Archimedes selbst giebt uns keine Aufklärung darüber, wie er diesen Schwerpunkt kennen gelernt hat, aber er nennt und benutzt ihn zu wiederholten Malen im 2ten Buche seiner Schrift über Körper, die auf einer Flüssigkeit schwimmen.

${ }^{1}$ Der Schluss des Beweises ist indessen im 7ten Abschnitte des überlieferten Textes durch ein offenbares Missverständnis von einem späteren Herausgeber auf ähnliche Segmente eingeschränkt worden. 
Im ersten Buche dieser hydrostatischen Schrift wird der bekannte Hauptsatz über das Gleichgewicht ganz oder teilweise eingtauchter Körper, der den Namen das archimedische Princip erhalten hat, aufgestellt und begründet. In diesem Buche nimmt Archimedes sogar einen so allgemeinen Standpunkt ein, dass er die Kugelgestalt der Erde und die Richtung der Schwere gegen ihren Mittelpunkt hin berücksichtigt. Im letzten Satze des Buches behandelt er die Aufgabe, die Gleichgewichtslage eines teilweise eingetauchten Kugelsegmentes zu finden; leider aber sind wesentliche Teile dieser Untersuchung verloren gegangen.

Dass Archimedes nicht ohne weiteres und nur aus Gründen der Symmetrie schliesst, dass es keine anderen Gleichgewichtslagen giebt als diejenige, bei der die Axe des Segmentes lotrecht steht, lässt sich vermuten aus der vollständigeren Behandlung, der er im 2ten Buche die Aufgabe unterwirft, die Gleichgewichtslagen eines Segmentes zu finden, das von einem Umdrehungsparaboloid durch einen senkrecht zur Axe gelegten Schnitt abgeschnitten wird. Bei dieser Untersuchung wird der Wasserspiegel jedoch als eben vorausgesetzt; bei ihr benutzt er die Schwerpunkte auch von solchen Segmenten, die durch schief zur Axe stehende Ebenen abgeschnitten werden.

Archimedes' statische Werke bilden die Grundlage sowohl für die theoretische Mechanik wie für die praktischen Anwendungen der Mechanik. Dass er es selbst in solchen Anwendungen weit brachte, wissen wir aus vielen Berichten des späteren Altertums, wo man es besser verstand die sichtbaren Resultate zu bewundern als die wissenschaftliche Arbeit. Eine unmittelbare Benutzung des archimedischen Princips ist seine Benutzung des specifischen Gewichtes um die Zusammensetzung von Metall- 
gemischen zu bestimmen («Hiero's Krone»). Er soll Apparate konstruiert haben um durch geringe Kraft grosse Massen zu bewegen. Die Wasserschnecke rührt von ihm her. Besondere Bedeutung erhielt seine mechanische Geschicklichkeit durch die während der Belagerung von Syrakus konstruierten Kriegsmaschinen; unter diesen ist jedoch die Anwendung des Brennspiegels, um die römische Flotte anzuzünden, eine Fabel, wenn es auch keineswegs unmöglich ist, dass er parabolische Brennspiegel erfunden hat. Mit grosser Bewunderung nannte man im Altertum ein von ihm konstruiertes mechanisches Planetarium.

\section{Die Lehre von den Kegelschnitten vor Apollonius.}

Wir haben bereits (S. 87) bei unserer Erwähnung des delischen Problems, sowie bei der Konstruktion von zwei mittleren Proportionalen angeführt, dass dieses Problem von Menächmus, dem Schüler des Eudoxus, gelöst wurde mittels der Schnittpunkte zwischen zwei von den Kurven

$$
a y=x^{2}, b x=y^{2}, x y=a b,
$$

die er als Schnittkurven zwischen einem Umdrehungskegel und einer Ebene darstellte. In Übereinstimmung hiermit wird berichtet, dass Menächmus die Kegelschnitte erfunden habe. Zugleich weiss man, dass man zu ihrer Herstellung bis zu Apollonius hinauf eine Ebene gebrauchte, die senkrecht auf einer Erzeugenden der Kegelfläche stand, wodurch Ellipsen, Parabeln und $\mathrm{Hy}$ perbeln die Namen "Schnitt des spitzwinkeligen, rechtwinkeligen und stumpfwinkeligen Kegels» erhielten. Hieraus darf man jedoch nicht schliessen, dass 
Menächmus und die Mathematiker bis zu Apollonius ein Verfahren besessen hätten, das besonders zu der Bestimmung von Eigenschaften solcher Schnitte führte, die senkrecht auf einer Erzeugenden standen, und nicht mit derselben Leichtigkeit benutzt werden konnte um nachzuweisen, dass anders belegene Schnitte ganz dieselben Eigenschaften besässen. Von einem solchen Verfahren ist uns keine Spur in der griechischen Mathematik aufbewahrt, und es dürfte, wenigstens was die Ellipse und Hyperbel betrifft, schwierig sein ein solches Verfahren $\mathrm{zu}$ $\mathrm{zu}$ erfinden.

Die Sache lässt sich indessen folgendermassen erklären. Für die Bestimmung der beiden mittleren Proportionalen liessen sich die Kurven verwenden, die durch die oben genannten Gleichungen definiert werden. Eine solche Definition musste indessen von dem Postulat begleitet sein, dass die Kurven wirklich existierten, oder mit anderen Worten, dass die durch diese Definition bestimmten Punkte kontinuierlich aufeinander folgten. Das Postulat konnte vermieden werden, wenn sich eine, auf frühere Postulate gegründete, Konstruktion der Kurven angeben liess, - ja in diesem Falle würde es sogar unstatthaft gewesen sein, neue Postulate aufzustellen. Die von $\mathrm{Me}$ nächmus gefundene Konstruktion besteht eben in der Bestimmung der Kurven als Schnitte an Kreiskegeln: die Kontinuität der Kegelfläche ist dann sichergestellt durch diejenige der Leitkurve, des Kreises, und die Kontinuität der Schnittkurve wiederum durch diejenige der Kegelfäche.

Für diese Verwendung ist jede Erzeugung als Schnitte an Kegeln gleich gut, ja um sich zu versichern, dass eine Kurve mit bestimmten Konstanten sich wirklich als Schnitt an einem Kegel erzeugen lässt, ist es sogar am bequemsten, wenn man eine gleichartige Lösung dieser Aufgabe hat. Dass dann namentlich die Lösung, bei der 
23. Die Lehre von den Kegelschnitten vor Apollonius. 193

man zugleich die Kegelfläche gerade und die Schnittebene senkrecht auf einer Erzeugenden sein lässt, zweckmässig sein kann, ergiebt sich leicht, wenn wir zuerst parabolische Schnitte betrachten, die als Schnitte am rechtwinkeligen Kegel hervorgebracht sind. $T$ sei der Scheitelpunkt einer solchen, $K T C$ ein Axenschnitt, $G P H$ die Spur eines parallel zur Grundfläche gelegten Schnittes, $y$ das

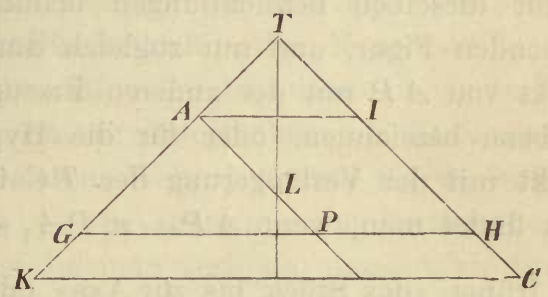

Stück, welches zwischen $P$ und der Kegelfläche auf einer Geraden abgeschnitten wird, die in $P$ senkrecht auf der Ebene der Figur steht. Dann ist, wenn $A P \perp T K$,

$$
y^{2}=G P . P H=\sqrt{2} . A P . A I=2 A P . A L .
$$

Der Schnitt, der in $A P$ senkrecht zur Ebene der Figur errichtet wird, wird dann, wenn $A P=x$ und $2 A L=p$, dargestellt durch

$$
y^{2}=p x
$$

In Übereinstimmung mit dieser Konstruktion wird der halbe Parameter $\frac{p}{2}$ noch von Archimedes «das Stück bis zur Axe» genannt, nämlich vom Scheitelpunkt $A$ der Parabel bis zur Axe des Kegels.

Man sieht also, dass Menächmus eben die Lösung derjenigen Aufgabe erhielt, die vorlag: eine Kurve mit der Gleichung $y^{2}=p x$ als Schnitt an einem Kegel darzustellen. Es kam nur darauf an, den Kegel gerade sein 
$\mathrm{zu}$ lassen, den Schnitt senkrecht zu einer Erzeugenden $\mathrm{zu}$ legen und dafür zu sorgen, dass «das Stück bis zur Axe» $\frac{p}{2}$ wurde.

Dieselben Vorteile erreicht man durch die entsprechende Darstellung der Ellipse und Hyperbel als Schnitte, die senkrecht auf der Erzeugenden eines spitzwinkeligen oder stumpfwinkeligen Umdrehungskegels stehen. Wir wollen dafür dieselben Bezeichnungen benutzen wie in der umstehenden Figur, und nur zugleich durch $A_{1}$ den Schnittpunkt von $A P$ mit der anderen Erzeugenden $T C$ der Figurebene bezeichnen, oder für die Hyperbel, den Schnittpunkt mit der Verlängerung der $T C$ über $T$ hinaus. Dann findet man, wenn $A P=x, P A_{1}=x_{1}$, wenn ferner wie früher "das Stück bis zur Axe» oder $A L=\frac{p}{2}$ der halbe Parameter ist, und wenn $A A_{1}=2 a$,

$$
y^{2}=\frac{2 A L}{A A_{1}} \cdot A P . P A_{1}=\frac{p}{2 a} x x_{1} .
$$

Diese Bestimmung (durch die Axengleichung) ist gerade diejenige, welche die ältesten griechischen Geometer in der einen oder anderen Form (ihre Bestimmung kommt der Gleichung $\frac{y^{2}}{x x_{1}}=\frac{p}{2 a}$ am nächsten) der Untersuchung der Ellipse und Hyperbel (je nachdem $x+x_{1}=2 a$, oder $x_{1}-x=2 a$ ) zu Grunde legen. Da die Konstanten der Kurve auf einfache Weise in der Figur dargestellt sind, so hat man in der That eine gute und bestimmte Methode diese Kurven als Schnitte an Kegeln darzustellen, also zu zeigen, dass sie für alle Werte der Konstanten Kegelschnitte sind.

Hierbei wird jedoch vorausgesetzt, dass diese Kurven vorher bekannt gewesen und - selbstverständlich unter 
23. Die Lehre von den Kegelschnitten vor Apollonius. 195

geometrischer Form - durch die angeführte Gleichung dargestellt worden sind. Was die Ellipse betrifft, so deutet verschiedenes hierauf; es kann dann sehr nahe gelegen haben, sie als Cylinderschnitt zu betrachten. Die Hyperbel, und namentlich die gleichseitige, hat sich, wie schon angeführt, als verwendbar für die Konstruktion von zwei mittleren Proportionalen gezeigt, aber freilich durch eine andere Gleichung bestimmt, nämlich durch diejenige, wodurch sie auf ihre Asymptoten bezogen wird. Die Verwendbarkeit für die Konstruktion der beiden mittleren Proportionalen hat dann eine gute Veranlassung gegeben, - um zu untersuchen, ob die Kurve nicht etwa eine auf anderem Wege bekannte Kurve, z. B. ein Kreis, sein könne - die ursprüngliche Bestimmung durch die Mittel, über die man verfügte, namentlich durch die geometrische Algebra, umzuformen. Die Umformung in die Axengleichung hat dann gerade für dieses Hülfsmittel ziemlich nahe gelegen. Ein unmittelbarer Zusammenhang zwischen der Asymptotengleichung und der Darstellung als Kegelschnitt ist aber kaum bekannt gewesen.

Das, was durch senkrechte Schnitte auf der Erzeugenden eines Umdrehungskegels erreicht wurde, war also das Mittel, jede Parabel, Ellipse oder Hyperbel als Schnitt eines Umdrehungskegels darzustellen. Selbstverständlich sah man zugleich, dass umgekehrt alle derartig angebrachten Schnitte Parabeln, Ellipsen und Hyperbeln sind. Es hat zugleich unmöglich der Aufmerksamkeit entgehen können, dass die besondere Lage durchaus gar keine Rolle bei dieser umgekehrten Bestimmung spielt. Jedenfalls hat dieselbe Bestimmungsmethode sich als anwendbar gezeigt, sobald man überhaupt Veranlassung fand nach anders belegenen Schnitten zu fragen. Das finden wir bestätigt in Archimedes' Schrift über Konoide und Sphäroide. Bereits die Einleitung zu dieser Schrift zeigt, 
dass man sogar vor seiner Zeit wenigstens alle elliptischen Schnitte an geraden Kegeln kannte, und im Verlaufe seiner eigenen Untersuchungen werden sogar gewisse ellip. tische Schnitte an schiefen Kreiskegeln betrachtet, nämlich solche, die senkrecht auf der Symmetrieebene des Kegels stehen. Archimedes löst die Aufgabe, die nach unserer Ausdrucksweise heissen würde: Bestimmung der cirkulären Schnitte an einer Kegelfläche zweiter Ordnung mit bekannten Hauptschnitten. Da Archimedes bei den vorliegenden Aufgaben keine Verwendung für hyperbolische Schnitte hat, die auf die angeführte Weise liegen, so darf man aus seinem Schweigen nicht schliessen, dass er sie nicht gekannt habe.

Archimedes erhält sogar Gelegenheit uns das Hülfsmittel kennen zu lehren, wodurch man die planimetrische Bestimmung ebener Schnitte an Kreiskegeln fand. Die

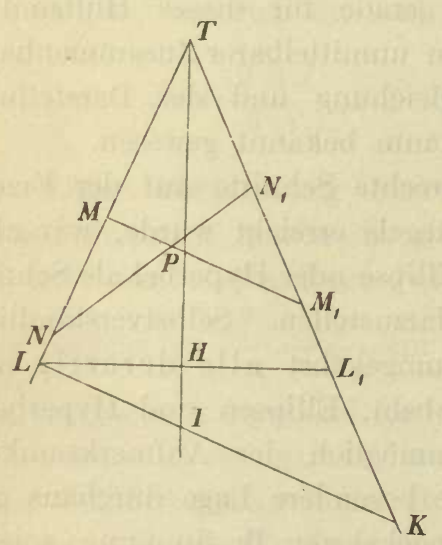

Figur möge diejenige sein, in der der Kegel von seiner Symmetrieebene geschnitten wird (oder, wenn der Kegel gerade ist, ein beliebiger Axenschnitt), $T L$ und $T K$ die in dieser Ebene liegenden Erzeugenden, $L K$ die Spur der kreisförmigen Grundfläche. Die Beschaffenheit des ebenen Schnittes, der in $N N_{1}$ projiciert wird, ergiebt sich dann durch folgenden planimetrischen Hülfssatz: wenn die Geraden $N N_{1}$ und $M M_{1}$, welche die festen geraden Linien $L T$ und $T K$ in $M$ und $N$, und in $M_{1}$ und $N_{1}$, sowie sich selbst in $P$ schneiden, ihre Richtungen nicht 
23. Die Lehre von den Kegelschnitten vor Apollonius. 197

verändern, so ist $\frac{P M \cdot P M_{1}}{P N \cdot P N_{1}}$ konstant oder gleich $k$. Ist nun $M M_{1}$ die Spur eines der Grundfläche parallelen Schnittes, und $y$ das Stück, welches von der in $P$ projicierten Geraden zwischen $P$ und der Kegelfläche abgeschnitten wird, so ist

$$
y^{2}=P M \cdot P M_{1}=k \cdot P N \cdot P N_{1},
$$

und das ist eben die Eigenschaft, durch die wir eine Ellipse oder Hyperbel charakterisiert haben.

Der hier benutzte planimetrische Satz wird als bekannt vorausgesetzt, ist also gewiss auch vor Archimedes benutzt worden um die Eigenschaften der Kegelschnitte abzuleiten. Er gilt nach dem sogenannten Potenzsatz, den wir später besprechen werden und der dem Archimedes bekannt war, auch wenn die Punkte $M, N, M_{1}$ und $N_{1}$ auf einem beliebigen Kegelschnitt liegen. A rchimedes konnte deshalb in der angeführten Schrift über Umdrehungsflächen zweiter Ordnung ebene Schnitte an diesen auf ganz dieselbe Weise bestimmen.

Wenn wir angenommen haben, dass die Entdeckung des Menächmus wesentlich darin bestand, dass Parabel, Ellipse und Hyperbel sich als Kegelschnitte darstellen liessen, so haben wir damit die Annahme verbinden müssen, dass diese Kurven wenigstens teilweise vorher untersucht worden waren, namentlich in Verbindung mit dem delischen Problem und auf Grundlage der Eigenschaften, die jetzt durch ihre einfachsten Gleichungen dargestellt werden. Diese Annahme wird in hohem Grade durch den Umstand unterstützt, dass es in allen weitergehenden Untersuchungen bei griechischen Schriftstellern die planimetrischen Haupteigenschaften sind und nicht die Darstellung als Kegelschnitte, die der Untersuchung zu Grunde gelegt werden. Sie trägt auch dazu bei zu 
erklären, dass die Lehre von den Kegelschnitten gleich nach Menächmus sich so rasch bei den Griechen entwickeln konnte, wie es der Fall war. Das Interesse für diese Lehre musste dadurch wachsen, dass man bald wahrnehmen musste, dass die Kegelschnitte sich nicht nur wie von Menächmus für die Konstruktion der beiden mittleren Proportionalen verwenden liessen, sondern auch bei der Lösung von vielen anderen Aufgaben, die man vergebens mit Hülfe von Zirkel und Lineal zu lösen versucht hatte. $\mathrm{Zu}$ dem Behufe musste man die Kegelschnitte als geometrische Örter benutzen, als "räumliche Örter», wie man sie damals nannte. Das Gewicht, das man auf eine solche Anwendung legte, zeigt sich dadurch, dass das älteste Werk über Kegelschnitte, das angeführt wird, den Titel «räumliche Örter» hatte. Es rührte von einem Mathematiker Aristäus her, der ein älterer Zeitgenosse von Euklid war. Dass der Titel dieses verlorenen Werkes wirklich eine besondere Bedeutung hatte und nicht nur ein Name für die Lehre von den Kegelschnitten im allgemeinen war, geht daraus hervor, dass die bald nachher erschienenen Bücher über Kegelschnitte von Euklid die räumlichen Örter des Aristäus ergänzen und nicht vertreten sollten. Man fuhr sogar fort diese Schrift neben den Kegelschnitten des Apollonius, welche diejenigen des Euklid vollständig verdrängten, zu studieren und zu benutzen.

Der Gebrauch, den Aristäus wahrscheinlich von den Kegelschnitten gemacht hat, und den man in weitergehendem Maasse gemacht hat, als die Lehre von den Kegelschnitten durch Euklid und Apollonius weiter entwickelt worden war, wird sich am besten verstehen lassen, wenn wir aus dem grossen Werk des Apollonius gelernt haben werden, wie die Alten diese Kurven überhaupt бehandelten. Aus diesem Werke werden wir zu- 
23. Die Lehre von den Kegelschnitten vor Apollonius. 199

gleich, wenn wir besonders darauf aufmerksam machen, welche Fortschritte man Apollonius persönlich verdankt, eine Vorstellung von dem gewinnen, was bereits Euklids Darstellung enthalten haben muss. Vorläufig wollen wir nur bemerken, dass sich aus Archimedes' Schriften erkennen lässt, dass dies nicht so ganz wenig gewesen sein kann; denn die Sätze über Kegelschnitte, die Archimedes als bekannt voraussetzt, sind gewiss in Euklids verlorenem Werke $\mathrm{zu}$ finden gewesen. In diesem muss man nicht nur die bereits angeführte Beziehung der Kegelschnitte auf ihre Axen haben finden können, sowie die daran angeschlossene Bestimmung von Tangenten, konjugierten Durchmessern und Asymptoten, sondern auch die entsprechende Beziehung auf zwei konjugierte Durchmesser, und die bereits dem Menächmus bekannte Beziehung auf die Asymptoten; endlich auch den soeben erwähnten Potenzsatz.

\section{Die Kegelschnitte des Apollonius.}

Aus Apollonius' grossem Werke über die Kegelschnitte kennen wir hauptsächlich die Lehre der Alten von den Kegelschnitten, so wie wir ihre elementare Geometrie aus Euklid kennen. Von Apollonius' 8 Büchern über Kegelschnitte sind uns, jedoch nur 7 erhalten geblieben, die 4 ersten auf Griechisch, die 3 letzten durch eine arabische Übersetzung. Die 4 ersten Bücher enthalten das, was man die Elemente der Lehre von den Kegelschnitten nennt, das heisst die zusammenhängende Darstellung der Haupteigenschaften der Kegelschnitte, die man zum Ausgangspunkt nehmen muss sowohl für die Anwendung der Lehre von den Kegelschnitten auf die Lösung von Konstruktionsaufgaben mittels räum- 
licher Örter, als auch für weitergehende besondere Untersuchungen einzelner, die Kegelschritte betreffender Fragen. Die folgenden Bücher dagegen enthalten hierher gehörige Specialuntersuchungen. Das fünfte Buch, das über Normalen an die Kegelschnitte handelt, ist zugleich das vollständigste erhaltene Beispiel für die Anwendung der Kegelschnitte auf Konstruktion, nämlich auf die Konstruktion von Normalen, die von einem gegebenen Punkte ausgehen, und für die an eine solche Konstruktion angeschlossene feinere theoretische Untersuchung.

Die allgemeine Kenntnis, welche die Alten von den Kegelschnitten hatten, lernen wir jedoch zunächst und vor allem aus den vier ersten Büchern kennen. Bei diesen wollen wir deshalb so lange verweilen, dass wir nicht nur einen Überblick über das zu geben vermögen, was die Alten über die Kegelschnitte wussten, sondern es auch verständlich machen, dass sie wirklich Mittel besassen um so weit zu gelangen.

Zunächst müssen wir dann sehen, wie im ersten Buche die Grundmauer aufgeführt wird. Geschieht dies auch mit einem anderen Ausgangspunkt, als der von Apollonius' Vorgängern benutzte war, so sind doch, wie sich aus seinen Vorreden ergiebt, die einzelnen Sätze, aus denen diese Grundmauer zusammengefügt ist, zum grossen Teile dieselben, die auch seine Vorgänger kannten und anwandten. Was die Hyperbel betrifft, so zeigt sich jedoch in diesen Sätzen der bedeutende Fortschritt, dass Apollonius, wenn er auch die beiden Äste der Hyperbel "zusammengehörende Hyperbeln» nennt, dennoch diese Äste als eine einzige Kurve behandelt, und dadurch die Gleichartigkeit in den Sätzen über Ellipse und Hyperbel erreicht, die nur auf diesem Wege möglich ist.

Der neue Ausgangspunkt besteht darin, dass Apol- 
lonius, statt Schnitte zu betrachten, die von Ebenen in einer bestimmten Lage an Umdrehungskegeln hervorgebracht werden, sofort beliebige ebene Schnitte an beliebigen Kreiskegeln betrachtet. Das Verfahren, welches benutzt wird um eine solche planimetrische Eigenschaft an diesen Schnitten abzuleiten, dass sie der weiteren Untersuchung der Kurven zu Grunde gelegt werden kann, ist eine Verallgemeinerung desjenigen, das wir Archimedes haben benutzen sehen um Schnitte senkrecht zur Symmetrieebene des Kegels zu untersuchen; dadurch wird aber die planimetrische Eigenschaft auch allgemeiner. Es wird dann nicht mehr diejenige, die dadurch ausgedrückt wird, dass man den Kegelschnitt auf eine von seinen Axen und die halben zugehörigen Sehnen als Ordinaten bezieht, sondern diejenige, die man auf ähnliche Weise erhält, indem man den Kegelschnitt auf einen beliebigen Durchmesser und dessen Sehnen bezieht. Das muss man festhalten, wenn man den rechten Überblick über den Gang des Buches erhalten will. Von den untersuchten Kurven ist es anfangs nur bekannt, dass sie diese Eigenschaft besitzen in Bezug auf einen einzelnen Durchmesser und ein dazu gehöriges Sehnensystem, das im allgemeinen einen schiefen Winkel mit dem Durchmesser bildet. Erst im weiteren Verlaufe der Darstellung sieht man, dass sie dieselbe Eigenschaft besitzen in Bezug auf unendlich viele Durchmesser, und am Schlusse des Buches werden endlich solche Durchmesser konstruiert, die senkrecht auf ihren Sehnen stehen, und gezeigt, dass die Kurven, wenn man sie auf diese bezogen hat, sich als Schnitte in Umdrehungskegel hineinlegen lassen. Erst dann ist die Identität der von A pollonius behandelten Kurven mit den bereits früher bekannten Kegelschnitten vollkommen dargethan.

Diese letzten Resultate machen also das Ziel aus, 
das Apollonius während der Aufführung des ganzen ersten Buches vor Augen gehabt hat. Während des Aufbaus ist er jedoch teils genötigt gewesen, teils hat er sich die Gelegenheit dazu verschafft, viele Eigenschaften der Kurven darzustellen, die in den späteren Büchern zur Verwendung gelangen können, oder die es an und für sich wert sind, dass man sie kennen lernt. So wird die

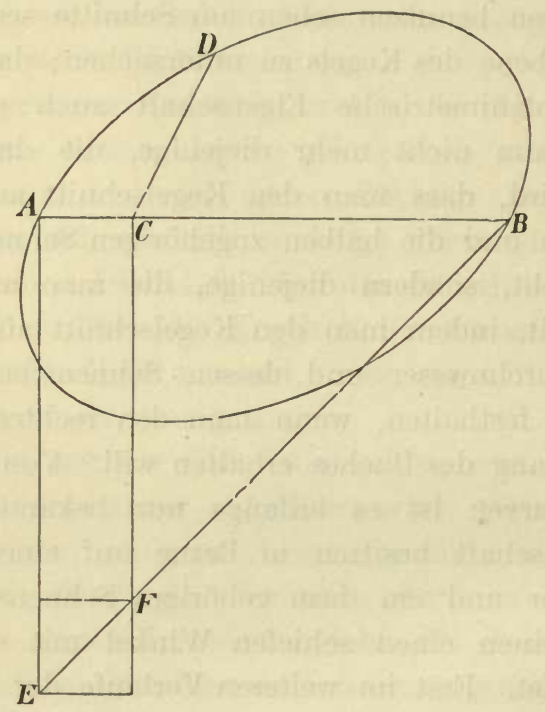

Lehre von den Tangenten und ihrer Bestimmung mitgenommen als Einleitung zu der, für den Hauptzweck des Buches notwendigen, Lehre von den Durchmessern $\mathrm{zu}$ parallelen Sehnensystemen.

Will man sich eine Vorstellung von den benutzten Arten des Verfahrens machen, so geschieht das am besten durch Vergleichung mit der, in der jetzigen analytischen Geometrie gebräuchlichen, algebraischen Umformung der Gleichungen in die neuen Formen, die man durch 
Beziehung auf neue Koordinatensysteme erhält, oder überhaupt mit den algebraischen Operationen der analytischen Geometrie. Diese werden nur von Apollonius mit Hülfe der geometrischen Algebra dargestellt. Die Brauchbarkeit dieser hierfür wird man am besten erkennen, wenn man die geometrische Form sieht, unter der Apollonius diejenigen Gleichungen für die Kegelschnitte darstellte, die er aus ihrer Lage auf dem Kegel ableitete. In diesen Gleichungen werden sie, wie schon gesagt, auf einen Durchmesser und die dazu gehörigen

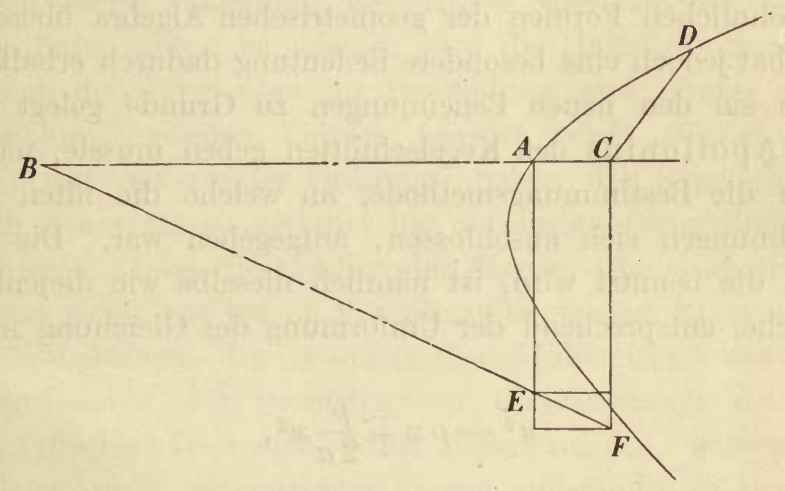

halben Sehnen als Ordinaten bezogen. $A B$ sei ein Durchmesser $2 a$ einer Ellipse oder Hyperbel, $C D$ die Hälfte einer dazu gehörigen Sehne. Dass dann das Quadrat $C D^{2}$ in einem konstanten Verhältnis $\frac{p}{2 a}$ zu dem Produkt $A C$. C B stehen soll, das findet seinen Ausdruck dadurch, dass man in $A$ und $C$ auf $A B$ die Senkrechten $A E$ und $C F$ errichtet, und auf der ersten $A E=p$ abträgt. Ist dann $F$ der Schnittpunkt zwischen $C F$ und $B E$, so muss das Quadrat über $C D$ dem Rechteck $A F$ gleich sein; denn $C F$ ist dann eben $\frac{p}{2 a} \cdot C B$. Die Hülfsfigur 
macht also den geometrisch-algebraischen Apparat aus, durch den man dasselbe darstellt, was wir, wenn $C D=y$ und $A C=x$, ausdrücken würden durch die Gleichung

$$
y^{2}=\frac{p}{2 a} x(2 a \mp x)
$$

Man sieht, dass die dargestellte Grundeigenschaft dieselbe ist, die man vor Apollonius kannte (und die man deshalb mit Unrecht das Theorem des Apollonius nennt). Die Darstellungsform stimmt durchaus mit den gewöhnlichen Formen der geometrischen Algebra überein. Sie hat jedoch eine besondere Bedeutung dadurch erhalten, dass sie den neuen Benennungen zu Grunde gelegt ist, die Apollonius den Kegelschnitten geben musste, nachdem die Bestimmungsmethode, an welche die alten Bezeichnungen sich anschlossen, aufgegeben war. Die Figur, die benutzt wird, ist nämlich dieselbe wie diejenige, welche, entsprechend der Unformung der Gleichung in

$$
y^{2}=p x \mp \frac{p}{2 a} x^{2}
$$

ausdrückt, dass das Quadrat $y^{2}$ an die Strecke $A E=p$ so angelegt ist, dass die Seiten des mangelnden oder überschiessenden Rechtecks sich wie $p: 2 a$ verhalten (vergl. Euklids 6tes Buch). In Übereinstimmung mit den bei der Auflösung der Gleichungen zweiten Grades benutzten Bezeichnungen wird die dargestellte Kurve selbst Ellipse oder Hyperbel genannt, je nachdem das Rechteck $E F$ mangelt oder überschiesst. Ist weder ein Überschuss noch ein Mangel vorhanden, so hat man eine einfache Anlegung des Quadrates $y^{2}$ an $p$. Die Kurve $y^{2}=p x$ hat dann denselben Namen Parabel bekommen, wie die einfache Flächenanlegung. 
Man sieht, dass die geometrische Algebra hier genau dieselben Dienste leistet, wie die Algebra in der späteren analytischen Geometrie. Wie wir jetzt die Grundeigenschaft einer Kurve durch eine algebraische Gleichung ausdrücken, so wird sie bei Apollonius durch eine Figur dargestellt. Dadurch dass diese Hülfsfigur rechtwinkelig zur Abscissenaxe gezeichnet ist, auch wenn die Ordinaten diese unter schiefen Winkeln schneiden, erhält sie eine gewisse Unabhängigkeit von der Figur, bei deren Untersuchung sie benutzt werden soll. Ebenso wie die algebraische Gleichung der Kurve vom zweiten Grade mit Bezug auf $x$ ist, ebenso wird die Hülfsfigur dieselbe, die in den Elementen zur Darstellung und Lösung einer Gleichung zweiten Grades benutzt wird. Gerade als "Kurven von zweiter Ordnung» haben die Kegelschnitte sich daher als so geeignet für die antike Behandlungsart erwiesen. Dass diese selbst der Algebra eine geometrische Form giebt, hat jedoch Veranlassung gegeben $\mathrm{zu}$ manchen Kombinationen des geometrischen Hülfsmittels mit dem Gegenstande der geometrischen Untersuchung, die der analytischen Geometrie ferner liegen würden, namentlich solange diese geometrische Fragen vollständig in Rechenaufgaben verwandelte. Im Gegensatze hierzu gleicht die antike Behandlung etwas mehr der jetzt üblichen Benutzung der analytischen Geometrie, bei der man die geometrische Bedeutung der vorzunehmenden Umformungen nicht vergisst.

Wir können allerdings hier nicht bis ins Einzelne die Umformungen der unter geometrischer Form dargestellten Gleichungen der Kurve verfolgen, wodurch man nach und nach zu dem Resultat gelangt, das wir als das Ziel des Buches bezeichnet haben, aber als Beispiel wollen wir doch ein Zwischenglied nennen, das eine Hauptrolle sowohl hier wie bei den weitergehenden Untersuchungen 
im 3ten Buche spielt. Ein Kegelschnitt mit dem Mittelpunkt $C$ und den Durchmessern $C E$ und $C B$ wird als geometrischer Ort für solche Punkte $H$ betrachtet, dass das Viereck $C M H T$, welches begrenzt wird von diesen beiden Durchmessern, von der Linie $H M$, die den von $C B$ halbierten Sehnen parallel ist, und von der $H T$, die den von $C E$ halbierten Sehnen parallel ist, einen konstanten Flächeninhalt erhält. Für uns ist dieser Flächensatz allgernein gültig,

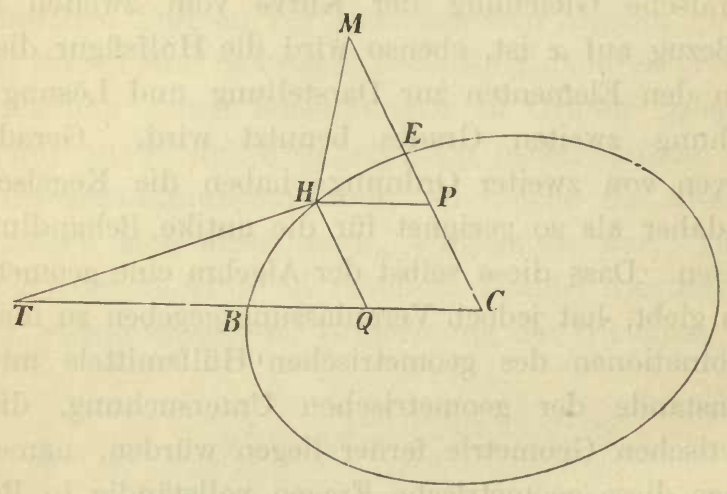

sobald wir die einzelnen Teile eines uneigentlichen Vierecks, wenn ein solches vorkommt, nach gewöhnlichen Regeln mit Vorzeichen rechnen. Apollonius dagegen muss ihn in mehrere verschiedene Sätze zerlegen, deren Zusammenhang er jedoch offenbar vor Augen hat. Die Anwendung, die Apollonius im ersten Buche von diesem Satze macht, wird man verstehen, wenn wir darauf hinweisen, dass der Satz zuerst aus der Gleichung abgeleitet wird, durch welche die Kurve auf den einen Durchmesser und seine Sehnen bezogen wird, und dass er darauf auf entsprechende Weise zu der Gleichung führt, wodurch 
die Kurve auf den anderen Durchmesser und seine Sehnen bezogen wird.

Aus dem ersten Buche wollen wir noch die Bestimmung der Tangenten erwähnen. Nach der Gleichung der Kurve kommt es bei dieser darauf an, durch einen Punkt $\left(x^{\prime}, y^{\prime}\right)$ der Kurve eine Gerade so zu ziehen, dass jeder andere Punkt $(x, y)$ von dieser der Bedingung

$$
\frac{y^{2}}{p x \mp \frac{p}{2 a} x^{2}}>\frac{y^{\prime 2}}{p x^{\prime} \mp \frac{p}{2 a} x^{\prime 2}}
$$

genügt, worin für die Parabel $\frac{p}{2 a}$ mit 0 vertauscht wird. Apollonius zeigt, dass dies für die Ellipse und Hyperbel erreicht wird, wenn die Tangente und die Ordinate im Punkte $\left(x^{\prime}, y^{\prime}\right)$ den Durchmesser harmonisch teilen (die Bezeichnung harmonisch ist jedoch neueren Ursprungs). Der Beweis ist etwas zu weitläufig, um hier wiederholt zu werden. Dagegen lässt sich der Beweis dafür, dass eine, von einem Punkte $\left(x^{\prime}, y^{\prime}\right)$ der Parabel $y^{2}=p x$ gezogene Gerade, welche die Abscissenaxe im Punkte $\left(-x^{\prime}, 0\right)$ trifft, Tangente der Parabel ist, sich etwa auf folgende Weise wiedergeben. Ist $(x, y)$ ein Punkt dieser Geraden, so wird

$$
\frac{y^{2}}{\left(x^{\prime}+x\right)^{2}}=\frac{y^{\prime 2}}{4 x^{\prime 2}}
$$

Da man nun durch Euklid weiss, dass die mittlere Proportionale zwischen zwei Grössen (ihr geometrisches Mittel) kleiner ist als ihr arithmetisches Mittel, oder dass

$$
x^{\prime} x<\left(\frac{x+x^{\prime}}{2}\right)^{2} \text {, so wird } \frac{y^{2}}{x}>\frac{y^{\prime 2}}{x^{\prime}} .
$$


Wenn man recht erkennt, wie gut und vollständig der Grund in Apollonius' erstem Buche gelegt ist, so versteht man um so mehr, wie er sich in mehreren von den übrigen Büchern so hoch erheben kann, namentlich im 3ten und zumteil im 5ten Buche. Wir müssen uns hier damit begnügen, den Inhalt dieser verschiedenen Bücher in aller Kürze anzugeben.

Im 2ten Buche werden die Haupteigenschaften der Asymptoten und konjugierten Durchmesser auseinandergesetzt. Ausser den zusammengehörenden Ästen einer Hyperbel werden auch konjugier te Hyperbeln betrachtet, die in verschiedenen Winkeln zwischen denselben Asymptoten liegen und Durchmesser von gleicher Länge haben. Es werden nämlich auch den Durchmessern, welche die Kurven nicht schneiden, Längen beigelegt, die in Wirklichkeit dieselben sind, die wir jetzt benutzen. Ausserdem werden verschiedene Aufgaben über Durchmesser und Asymptoten gelöst, darunter die Konstruktion von Mittelpunkt und Axen eines gegebenen Kegelschnittes, Konstruktion einer Tangente, die einen gegebenen Winkel mit dem Berührungsdurchmesser bildet u. s. w.

Das 3te Buch enthält vor allem solche Sätze, welche sich auf die Punkte der Kurven unabhängig von Durchmessern und Axen beziehen. Der Ableitung dieser Sätze wird der schon genannte Flächensatz zu Grunde gelegt, der in Wirklichkeit eine Beziehung der Kurve auf zwei nicht konjugierte Durchmesser darstellt. Man begreift, dass er auch einen guten Ausgangspunkt für den Beweis des auch von Archimedes gekannten Potenzsatzes abgeben kann; denn dieser betrifft Sehnen mit gegebenen, aber willkürlich gewählten Richtungen. Die Hauptsätze über Pol und Polare finden sich auch vor. Endlich kommt auch die Erzeugung eines Kegelschnittes durch zwei solche Geradenbüschel vor, die man jetzt pro- 
jektivische nennt. Die Scheitel der Büschel sind beliebige Punkte $A$ und $C$ der Kurve, und die einander entsprechenden Geraden $A M$ und $C M$ werden dadurch bestimmt, dass sie auf den, zu den Tangenten in $A$ und $C$ gezogenen Parallelen die Stücke $C P$ und $A Q$ abschneiden, welche ein Rechteck von konstantem Flächeninhalt bilden.

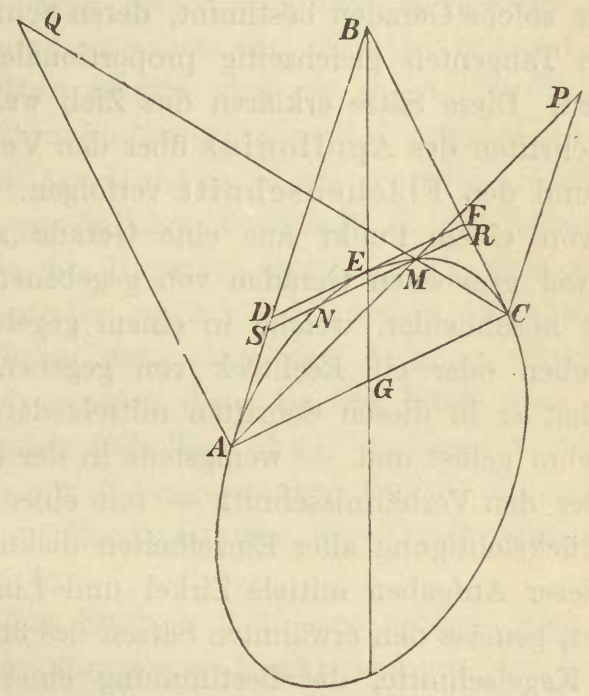

Man sieht leicht, dass alle diese Sätze unvollständig und wenig übersichtlich werden, wenn ein einzelner $\mathrm{Hy}$ perbelast allein für sich betrachtet wird. Man versteht deshalb, dass Apollonius' neue Auffassung der beiden Kurvenäste namentlich seinem drittem Buche einen wesentlichen Vorzug vor der früheren Behandlung desselben Gegenstandes verliehen hat, selbst wenn einzelne Sätze in begrenzterer Form vorher bekannt gewesen sind.

Eine andere Reihe von Sätzen desselben Buches enthält die einfachsten Bestimmungen von Tangenten ohne 
Benutzung der Berührungspunkte, namentlich von Tangenten an eine Hyperbel als denjenigen Geraden, die auf den Asymptoten vom Mittelpunkt aus - und von Tangenten an eine Ellipse und Hyperbel als denjenigen Geraden, die auf parallelen Tangenten vom Berührungspunkte aus - Stücke abschneiden, die ein Rechteck von konstantem Inhalt bilden. Tangenten an eine Parabel werden als solche Geraden bestimmt, deren Schnittpunkte mit festen Tangenten gleichzeitig proportionale Strecken durchlaufen. Diese Sätze erklären das Ziel, welches zwei kleinere Schriften des Apollonius über den Verhältnisschnitt und den Flächenschnitt verfolgen. Die Aufgaben: "von einem Punkt aus eine Gerade zu ziehen, die auf zwei gegebenen Geraden von gegebenen Punkten an Stücke abschneidet, welche in einem gegebenen Verhältnis stehen oder ein Rechteck von gegebener Fläche bilden," hat er in diesen Schriften mittels der geometrischen Algebra gelöst und - wenigstens in der erhaltenen Schrift über den Verhältnisschnitt - mit einer fast peinlichen Berücksichtigung aller Einzelheiten diskutiert. Die Lösung dieser Aufgaben mittels Zirkel und Lineal liefert in der That, gemäss den erwähnten Sätzen des 3ten Buches über die Kegelschnitte, die Bestimmung einer Tangente von einem gegebenen Punkte an einen hinlänglich bestimmten Kegelschnitt.

Noch ein bemerkenswerter kleiner Abschnitt ist in demselben dritten Buche enthalten, nämlich eine elementar-geometrische Behandlung der Lehre von den Brennpunkten der Ellipse und Hyperbel. Die Lage dieser Punkte $F$ und $F_{1}$ auf der Hauptaxe $A A_{1}$ ist, wie angegeben wird, dadurch bestimmt, dass das Rechteck $A F . F A_{1}$ gleich $\frac{1}{2} a p$ sein muss, wo $2 a$ und $p$ die Länge der Axe und des Parameters bezeichnen. Mit Hülfe der eben genannten Bestimmung von Tangenten an die Ellipse 
und Hyperbel ergiebt sich dann, dass das Stück, welches die Tangenten in $A$ und $A_{1}$ auf einer beweglichen Tangente abschneiden, von $F$ und $F_{1}$ aus unter einem rechten Winkel gesehen wird, wonach die übrigen Hauptsätze leicht $\mathrm{zu}$ erreichen sind. Über den Brennpunkt der $\mathrm{Pa}$ rabel findet sich merkwürdigerweise nichts bei Apollonius. Pappus' Hülfssätze zu einer verlorenen Schrift Euklids lassen jedoch vermuten, dass dieser Punkt teilweise wenigstens schon dem Euklid bekannt war.

Im 4ten Buche wird die grösste Anzahl (Maximum) der Schnittpunkte zwischen zwei Kegelschnitten bestimmt. Hier giebt Apollonius in der Vorrede ausdrücklich an, dass sein persönlicher Fortschritt in der Bezugnahme auf beide Äste der Hyperbel besteht, ein Umstand, der hier eine Hauptrolle spielt. Bei unserer Auseinandersetzung über die Behandlung räumlicher Aufgaben durch die Alten werden wir genauer über das 5te Buch reden. Das 6te Buch handelt teils über ähnliche Kegelschnitte, teils enthält es einige Erweiterungen der im ersten Buche vorgenommenen Konstruktionen von Kegeln durch vorgelegte Kegelschnitte.

Das 7te Buch enthält eine grössere Sammlung von Ausdrücken für gewisse Funktionen von den Längen konjugierter Durchmesser, von den zugehörenden Parametern u. s. w. Namentlich kommen hier die wichtigen Sätze vor, dass der Inhalt desjenigen Dreiecks, welches von einem Paare konjugierter Durchmesser (die bei der $\mathrm{Hy}$ perbel Durchmesser konjugierter Hyperbeln sind) und der zwischen ihnen liegenden Sehne gebildet wird, konstant ist, ebenso wie die Summe oder Differenz der Quadrate von konjugierten Durchmessern. In den anderen Fällen, wo die Funktionen sich nicht als konstant erweisen, werden ihre Maximal- oder Minimalwerte gesucht. Da nun angegeben wird, dass das 7 te Buch die Beweise für die 
Diorismen der Aufgaben enthält, die in dem jetzt verlorenen 8ten Buch ihre Lösung fanden, so müssen diese Aufgaben darauf ausgegangen sein solche konjugierte Durchmesser $\mathrm{zu}$ finden, für welche diese Funktionen gegegebene Werten haben. Die Ausdrücke, welche im 7 ten Buche für diese Funktionen gefunden sind, haben sofort die für die Lösung der Aufgaben erforderlichen Gleichungen geliefert.

\section{Räumliche Örter und Aufgaben.}

Der ursprüngliche Zweck der Lehre von den Kegelschnitten bestand darin, wie wir bereits angegeben haben, geometrische Örter herzustellen, die sich bei der Lösung solcher Aufgaben benutzen liessen, bei denen die Gerade und der Kreis nicht mehr ausreichten. Aufgaben, die sich mit Hülfe der Geraden und des Kreises lösen lassen, heissen ebene Aufgaben, und Gerade und Kreis heissen als geometrische Örter ebene Örter. Im späteren Altertum nahm man an, dass der letzte von diesen Namen der ursprüngliche sei und daher rühre, dass die angeführten Linien ursprünglich als in einer Ebene liegend bestimmt werden. Es ist jedoch genau eben so wahrscheinlich, dass der Name eben ursprünglich solchen Aufgaben angehört hat, die von Gleichungen von höchstens dem zweiten Grade abhängen, also in der geometrischen Algebra in der Ebene durch Relationen zwischen Flächen dargestellt werden. Ist das richtig, so hat der Name «räumliche A ufgaben» ursprünglich solchen angehört, die von Gleichungen 3ten Grades abhängen und durch Relationen zwischen Parallelepipeden dargestellt werden. "Räumliche Örter» bezeichnen solche geometrische Örter, die Kegelschnitte sind. Man darf annehmen, dass dieser Name 
daher rührt, dass sie für die Lösung von räumlichen Aufgaben bestimmt waren. Bereits im späteren Altertum nahm man jedoch an, dass umgekehrt der Name räumliche Örter der ältere sei und von der stereometrischen Definition der Kegelschnitte herrühre.

Leider ist die Schrift des Aristäus, in der die Kegelschnitte namentlich als geometrische Örter behandelt worden sein sollen, verloren gegangen, und wir wissen nichts von späteren Werken, in denen dasselbe Ziel verfolgt ist. Da jedoch Apollonius' Lehre von den Kegelschnitten denselben Gegenstand von einer anderen Seite behandelt hat, so lässt sich daraus schliessen, welche «räumlichen Örter» man gekannt hat oder doch leicht hat finden können, sobald in einer Aufgabe Verwendung dafür war. Bereits aus Apollonius' erstem Buche sieht man, dass dies nicht nur der Fall gewesen sein muss, wenn gewisse gegebene Linien sich sofort als konjugierte Durchmesser oder dergleichen ergaben. Im Flächensatze wird nämlich die Kurve auf zwei nicht konjugierte Durchmesser bezogen. Das dritte Buch führt uns jedoch weiter, teils durch seinen eigenen allgemeineren Charakter, teils dadurch, dass Apollonius ausdrücklich angiebt, wozu es benutzt werden soll. Seine besondere, gewiss durch Einführung der beiden Hyperbeläste vervollständigte, Behandlungsart soll nämlich nach seiner eigenen Angabe den Mängeln bei der älteren Bestimmung räumlicher Örter abhelfen. Unter diesen Örtern wird ausdrücklich «der Ort zu drei oder vier Geraden» genannt. Der Ort zu vier Geraden ist diejenige Kurve, welche, wenn $x, y, z$ und $u$, gemessen auf Schiefen von gegebenen Richtungen, die Abstände eines Punktes von vier feșten Geraden bezeichnen und $k$ eine Konstante bedeutet, durch die Gleichung

$$
x z=k y u
$$


dargestellt wird. Der Ort zu drei Geraden wird auf ähnliche Weise durch die Gleichung

$$
x z=k y^{2}
$$

dargestellt.

Ältere, nicht ganz vollständige Beweise dafür, dass diese Örter Kegelschnitte sind, muss Apollonius als so wohl bekannt voraussetzen, dass seine Leser, ohne dass die Beweise wiederholt wurden, sehen konnten, dass sie durch sein 3tes Buch vervollständigt wurden. Dieses Buch muss also die Voraussetzungen vervollständigt haben, auf denen man vorher diese Beweise aufführte und auch fortfahren sollte sie aufzuführen. Wir, die wir die ältere Bestimmung der Örter nicht kennen, können nur aus dem, was im Buche vorliegt, versuchen Schlüsse auf diese zu ziehen. Das ist nicht so schwierig für den Ort zu drei Geraden. Dass ein beliebiger Kegelschnitt ein solcher Ort ist, leitet Apollonius nämlich selbst (im Verlaufe des Beweises für die erwähnte Erzeugung durch projektivische Büschel) ab aus einem speciellen Falle des Potenzsatzes. Dass er auch ein Ort zu vier Geraden sein kann, lässt sich in dem Falle, wo zwei gegenüberliegende Geraden, $y=0$ und $u=0$, parallel sind, aus dem allgemeinen Potenzsatz ableiten.

Dass schon hierdurch viel erreicht ist, sieht man am besten durch eine Zusammenstellung mit der Darstellung eines «räumlichen Ortes» durch die analytische Geometrie. Nimmt man einen Punkt dieses Ortes zum Anfangspunkt, so erhält man eine Gleichung von der Form

$$
a x^{2}+b x y+c y^{2}+d x+e y=0
$$

oder

$$
x(a x+b y+d)=-c y\left(y+\frac{e}{c}\right) .
$$


Die Kurve wird also geradezu als Ort zu vier Geraden dargestellt, von denen zwei gegenüberliegende parallel sind. Da nun die von den Alten vorgenommene Umlegung von Flächen und Einführung von Koefficienten mittels der Proportionslehre genau unserer Behandlung von Ausdrücken zweiten Grades entspricht, so wird die jetzige Darstellung einer Kurve durch eine allgemeine Gleichung zweiten Grades ziemlich genau der antiken Darstellung als Ort zu vier Geraden, von denen zwei gegenüberliegende parallel sind, in geometrischer Tragweite entsprochen haben. Hierauf liess sich auch der allgemeine Ort zu vier Geraden zurückführen. Dass man wirklich ein Auge für diese umfassende Bedeutung des Ortes zu vier Geraden hatte, geht aus dem Gewicht hervor, das Apollonius darauf legt, gerade die Behandlung dieses Ortes verbessert $\mathrm{zu}$ haben. Übrigens scheint auch Euklids verlorene Schrift über Porismen Mittel zu solchen Umformungen angegeben $\mathrm{zu}$ haben, wie sie hier Verwendung finden könnten.

Es giebt eine verlorene Schrift des Apollonius, die man mit dem Ort zu vier Geraden in Verbindung bringen könnte, nämlich die Schrift über den bestimmten Schnitt. Man weiss nämlich, dass diese die Konstruktion von Punkten auf einer Geraden enthalten hat, deren Abstände von zwei Punktepaaren derselben Geraden Rechtecke von gegebenem Verhältnis bilden, sowie eine sorgfältige Diskussion dieser Aufgabe. Auf diese Aufgabe wird die Bestimmung der Schnittpunkte zwischen einer Geraden und einem Ort zu vier Geraden zurückgeführt, wenn alle vier Abstände parallel mit der gegebenen Geraden gerechnet werden. Fasst man die Sache so auf, so fällt die Bestimmung eines Ortes zu vier Geraden zusamnen nit dem Satze über Involution der Schnittpunkte einer Geraden mit einem Kegelschnitte und mit den gegenüber- 
liegenden Seiten eines einbeschriebenen Vierecks, der später von Desargues wiedergefunden ist und seinen Namen trägt. Sicher ist es, dass die Schrift über den bestimmten Schnitt einzelne wichtige Teile der jetzigen Lehre von der Involution enthalten hat.

Wie soeben angeführt waren räumliche Aufgaben wohl ursprünglich solche, die von Gleichungen dritten Grades abhingen und stereometrisch dargestellt wurden. Später dagegen wurde der Name mit der Lösung durch Kegelschnitte verbunden, und dadurch kam es, dass er faktisch auch solche Aufgaben umfasste, die - wenn man sie auf eine Gleichung gebracht haben würde von Gleichungen vierten Grades abhängig gewesen wären.

Die einfachste räumliche Aufgabe, die reine kubische Gleichung, haben wir bereits in der Form der Frage nach der Multiplikation des Würfels kennen gelernt, und wir haben zugleich gesehen, wie die Benutzung von Kegelschnitten sich ursprünglich an die Lösung dieser Aufgabe knüpfte. Andere Beispiele sind uns begegnet in der Dreiteilung des Winkels oder in den Einschiebungen, worauf diese zurückgeführt wird, und wir haben erwähnt, dass Archimedes in der Schrift über die Spiralen einen anderen Gebrauch von derselben Einschiebungen macht. Wie diese durch Kegelschnitte ausgeführt wurden, das hat uns Pappus mitgeteilt.

Die wichtigsten Beispiele für die Lösung räumlicher Aufgaben durch Kegelschnitte, die wir aus den besten Tagen der griechischen Mathematik besitzen, finden sich jedoch in der überlieferten Behandlung der Gleichung, auf welche Archimedes seine Teilung der Kugel zurückführt (vergl. S. 185), und in der Konstruktion von Normalen von einem Punkte an einem Kegelschnitt im 5ten Buche des Apollonius. Was diesen Lösungen 
namentlich Interesse verleiht, ist die Sorgfalt, mit der sowohl die Bedingungen für die Möglichkeit auseinander gesetzt werden, sowie auch die verschiedene Anzahl von Lösungen, die man erhält, wenn man den gegebenen Grössen verschiedene Werte beilegt. Dadurch tritt - in voller Übereinstimmung mit dem, was wir über die Bedeutung der geometrischen Konstruktion bei den Griechen gesagt haben - deutlich hervor, dass die Konstruktion durch Kegelschnitte nicht so sehr ein Mittel - und zwar ein sehr dürftiges - ist um die gesuchten Grössen herzustellen, sondern vielmehr ein gutes theoretisches Mittel um zu untersuchen, in welchen Fällen sie existieren. Die Bestimmungen der Maxima und Minima, die man dadurch für die gegebenen Grössen erhält, sind die wirklichen und bedeutungsvollen geometrischen Sätze, die das Hauptziel der Untersuchung bildeten.

Wir haben gesehen, dass Archimedes die Kugelteilung zurückführte auf die Gleichung

$$
D B^{2}: D X^{2}=X Z: T Z \text {, }
$$

wo $D, B, T$ und $Z$ bekannte Punkte, $X$ ein gesuchter Punkt einer Geraden ist. In dem bereits erwähnten überlieferten Manuskript, das vielleicht von Archimedes selbst herrührt und einen Anhang zu seinem 2ten Buch über die Kugel und den Cylinder gebildet hat, wird diese Aufgabe auf eine Weise gelöst, die wir am leichtesten in modernen algebraischen Zeichen folgendermassen wiedergeben können. Schreiben wir seine Gleichung in der Form

$$
\frac{b^{2}}{x^{2}}=\frac{a-x}{c}
$$

und setzen wir diese beiden Quotienten gleich $\frac{e}{y}$, wo $e$ eine beliebige Gerade ist, so lassen sich $x$ und $y$ bestimmen 
als Schnittpunkte zwischen der Parabel $x^{2}=\frac{b^{2}}{e} \cdot y$ und der Hyperbel $(a-x) y=c e$.

Bei den Anwendungen auf die Kugelteilung sind die Konstanten, die wir hier mit $a$ und $c$ bezeichnet haben, positiv, und es kommt darauf an, einen solchen Wert von $x$ zu bestimmen, dass $0<x<\frac{2}{3} a$, da $X$ zwischen $D$ und $B$, den Endpunkten des Kugeldurchmessers $D B=\frac{2}{3} D Z$, fallen muss. Die erhaltene Darstellung der Gleichung begreift jedoch auf Grund der verschiedenen Lagen, die man $Z$ und $T$ erteilen kann, oder die man den gesuchten Punkt $X$ einnehmen lassen kann, alle Gleichungen von der Form

$$
x^{3}+a x^{2}+b=0
$$

in sich, und die Aufgabe lässt sich so stellen, dass man alle Wurzeln mit bekommt. Wir haben hier also ein Beispiel für das, was wir schon früher gesagt haben, dass nämlich, wenn die Griechen auch nicht unsere Benutzung der Vorzeichen kannten, die geometrische Darstellung diesen Mangel immerhin weniger fühlbar machte.

Was nun die Grenzbedingungen betrifft, so werden diese bei den einzelnen Aufgaben zum Teil darauf beruhen, ob der Punkt $X$ auf oder neben die Intervalle fällt, welche die vorliegende Aufgabe fordert; was aber zugleich bei allen diesen Aufgaben eine Hauptsache bleibt, das ist die Erkennung der Grenzfälle, in denen die Kegelschnitte sich berühren, zwei Wurzeln also zusammenfallen; denn dadurch wird der Übergang zwischen den Fällen gebildet, in denen diese beiden. Wurzeln reell sind, also nach der damals geltenden Auffassung wirklich existiren konnten oder nicht. In dem aufbewahrten Bruchstücke wird angegeben, dass dieser Übergangsfall eintritt, wenn $x=\frac{2}{3} a$, 
wenn also $b^{2} c=\frac{4}{27} a^{3}$. Ist dagegen $x<\frac{2}{3} a$, so muss $b^{2} c<\frac{4}{27} a^{3}$ sein, was also die Bedingung für zwei Auflösungen wird. Dies wird leicht bewiesen mit Hülfe der Sätze über die Tangenten der Kegelschnitte. Soll nämlich die Tangente der Parabel im Punkte $P$ die Hyperbel berühren, deren Asymptoten die Geraden $y=0$ und $y=a$ sind, so muss $P$ die Mitte des Stückes der Tangente sein, welches die Asymptoten abschneiden. Der Schnittpunkt $S$ der Tangente mit der Abscissenaxe, die Tangente im Scheitelpunkt der Parabel ist, soll ferner die Mitte zwischen $P$ und dem Schnittpunkt mit der Ordinatenaxe sein. Daraus ergiebt sich, dass $D Q$, die Abscisse von $P$, gleich $\frac{2}{3} D Z$ ist. Man überzeugt sich leicht,

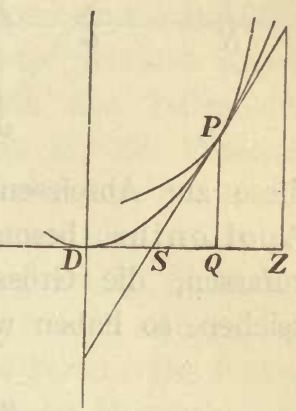
dass, wie Archimedes sagt, die für die Möglichkeit der Lösung gestellte Bedingung, $b^{2} c<\frac{4}{27} a^{3}$, wirklich bei der Kugelteilung erfüllt ist, bei der der Punkt $B$ auf $Q$, und $T$ zwischen $Q$ und $Z$ fällt. Man erhält jedoch nur eine Lösung, da die Punkte $X$ auf verschiedene Seiten von $B$ fallen würden, und man nur denjenigen benutzen kann, der auf $D B$ fällt.

Archimedes führt also seine Aufgabe über Kugelteilung zurück auf eine kubische Gleichung von sehr allgemeiner Form, die, wie bereits bemerkt wurde, auch einen Beweis liefert für den letzten Satz des zweiten Buches über die Kugel und den Cylinder, und die ferner Anwendung findet bei einigen von Archimedes an anderen Orten gestellten Aufgaben über die Bestimmung von Segmenten von Ellipsoiden und Hyperboloiden mit gegebenem Volumen. Apollonius' Bestimmung von Nor- 
malen ist dagegen ein Beispiel für eine Aufgabe, die direkt durch Kegelschnitte gelöst wird ohne Aufstellung einer Gleichung. Wir wollen uns damit begnügen seine Lö-

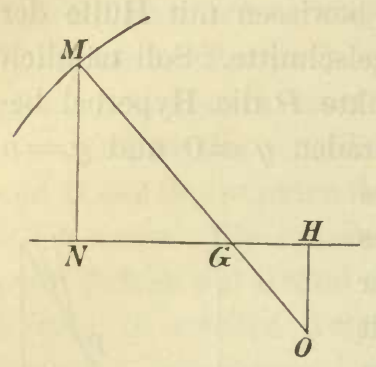
sung in der Sprache der jetzigen Algebra mitzuteilen. $O$ sei ein Punkt $\left(x_{1}, y_{1}\right)$ der an einen Punkt $M(x, y)$ eines Kegelschnittes gezogenen Normalen, $G$ der Schnittpunkt dieser Normalen mit der Hauptaxe, und $N$ die Projektion von $M$ auf dieselbe Axe. Nehmen wir dann diese zur Abscissenaxe und rechnen wir, um die von Apollonius besonders behandelten Fälle zusammenzufassen, die Grössen nach moderner Weise mit Vorzeichen, so haben wir

$$
\frac{y}{-y_{1}}=\frac{N G}{x_{1}-x-N G}
$$

$N G$ ist die Grösse, die wir jetzt die Subnormale nennen, und beträgt für die Parabel $\frac{p}{2}$; dadurch verwandelt sich die gefundene Gleichung in

$$
x y-\left(x_{1}-\frac{p}{2}\right) y-y_{1} \frac{p}{2}=0
$$

für die Ellipse und Hyperbel ist die Subnormale, wenn man den Mittelpunkt zum Anfangspunkt nimmt, beziehungsweise $\mp \frac{b^{2}}{a^{2}} x$, und dadurch verwandelt sich die Gleichung in

$$
\left(1 \mp \frac{b^{2}}{a^{2}}\right) x y-x_{1} y \pm \frac{b^{2}}{a^{2}} y_{1} x=0 .
$$


In beiden Fällen wird, wenn der Punkt $\left(x_{1}, y_{1}\right)$ ge. geben ist, der Punkt $(x, y)$ auf einer Hyperbel liegen, deren Schnittpunkte mit der gegebenen Kurve die Fusspunkte der vom Punkte $\left(x_{1}, y_{1}\right)$ ausgehenden Normalen sein werden. Diese Bestimmung der Normalen benutzt Apollonius um ausführlich zu untersuchen, wie viele Normalen sich von den verschiedenen Punkten der Ebene ziehen lassen. Die Hauptsache ist hier diejenigen Punkte zu bestimmen, deren entsprechende Hyperbeln die gegebene Kurve berühren. Die von diesen Punkten gebildete Kurve bildet nämlich den Übergang zwischen den Teilen der Ebene, die so beschaffen sind, dass man von den Punkten des einen Teiles zwei Normalen mehr ziehen kann als von denjenigen des anderen. Indem Apollonius die Bedingungen für die erwähnte Berührung sucht, findet er, wie man die Ordinate eines Punktes dieser Kurve bestimmen kann, wenn man die Abscisse kennt. Die Kurve ist dieselbe, die man jetzt die Evolute des Kegelschnittes nennt. Indessen ist weder bei Apollonius noch bei den Alten überhaupt die Rede von irgend einer genaueren Untersuchung dieser Kurve, als die hier angeführte ist.

\section{Die berechnende Geometrie.}

Aus Archimedes' Integrationen, wie wir sie genannt haben, aus Apollonius' Lehre von den Kegelschnitten und aus der von den Griechen gemachten Anwendung der Kegelsçhnitte zur Diskussion von Aufgaben, die in unserer Mathematik von Gleichungen dritten und vierten Grades abhängen, erkennen wir, bis zu welcher Höhe die griechische Geometrie und die Mathematik in geometrischer Form sich gehoben hatten. Wir haben früher gesehen, mit welcher Strenge man die Algemeingültigkeit dieser Mathematik 
sicher stellte. Wir haben indessen auch oft Gelegenheit gehabt zu erwähnen, dass man bei diesem Streben nach Algemeingültigkeit zu wenig Gewicht legte auf die Entwickelung der Hülfsmittel für wirkliche numerische Berechnung, durch welche die Mathematik praktische Anwendung erhalten konnte.

Selbstverständlich vernachlässigte man jedoch diese Seite der Sache nicht ganz. Man fuhr fort die geometrischen Sätze, die man von den ägyptischen Landmessern gelernt hatte, auf das Landmessen anzuwenden, und fügte dazu auch die Anwendung der einfachsten von den Sätzen, die man selbst fand. Es würde ungereimt sein anzunehmen, dass die Mathematiker, die Einsicht genug besassen um ihren Resultaten eine so allgemeingültige Form zu geben, nicht auch eben dadurch die specielle Anwendbarkeit dieser Resultate auf die numerischen Aufgaben kennen sollten, die in der Praxis vorkommen können. Denjenigen, welche eine so scharfsinnige Lehre von den Proportionen ausgearbeitet haben, haben die Mittel für die Behandlung solcher Aufgaben, die in der Praxis unter die einfache oder zusammengesetzte Regula de Tri gehören, nicht gefehlt. Hero - bei dem wir zugleich eines von den geometrischen Resultaten antreffen, von dessen Anwendung in der Praxis namentlich die Rede sein könnte, nämlich die Bestimmung des Inhaltes eines Dreiecks aus seinen Seiten - hat in seinen Aufgabensammlungen Zeugnis gegeben von der numerischen Anwendung wenigstens der einfachsten planimetrischen und stereometrischen Sätze und von der Auflösung von Gleichungen zweiten Grades. Das beschränkte Gebiet der Geometrie, aus dem diese Anwendungen genommen werden, und der bescheidene Grad von Genauigkeit, mit dem Hero sich bei seinen Berechnungen begnügt, deuten jedoch darauf hin, dass 
in der That guter Grund vorhanden ist, diese Seite der griechischen Mathematik etwas niedriger zu stellen.

Es war nicht ausschliesslich der schon (S. 57-61) erwähnte Mangel an Rechenfertigkeit, der in den besten Tagen der griechischen Geometrie ihrer Anwendung auf wirklicheBerechnungen Hindernisse entgegenstellte, sondern ihre Resultate waren für solche nicht besonders eingerichtet. Die Aufgaben werden in der Form von Konstruktionen gelöst, und diese lassen sich gewiss oft in Berechnung umsetzen, so wie man es sicher lange vor Hero gethan hat. Indessen giebt es, selbst wenn wir uns an die elementare Geometrie halten wollen, ein wichtiges Gebiet, auf dem diese Umsetzung sich nicht bewerkstelligen lässt, nämlich dasjenige, wo unter den Grössen, die sich durch einander bestimmen lassen sollen, nicht bloss Strecken, Flächen und Volumina vorkommen, sondern auch Winkel. Mit anderen Worten, die Griechen besassen in der besten alexandrinischen Zeit noch keine 'Trigonometrie, ein Mangel, dem erst die grossen Geometer und Astronomen jener Zeit abzuhelfen begannen. Bevor dies geschah, war man jedoch nicht ganz von solchen Untersuchungen ausgeschlossen, die man jetzt auf trigonometrischem Wege vornimmt. Die Sätze 12 und $13 \mathrm{im} 2$ ten Buche von Euklids Elementen drücken ganz dasselbe aus wie die Formel

$$
a^{2}=b^{2}+c^{2}-2 b c \cos \alpha
$$

und lassen sich ebenso ganz wie diese bei allen allgemeinen Untersuchungen anwenden, bei denen der Winkel a nicht gerade in Winkelmaass gegeben ist oder in solchem auszudrücken versucht wird. Wie scharf man den Zusammenhang zwischen der Grösse von Winkeln und dem Verhältnisse von Strecken auffasste, geht aus den Sätzen in Euklids Data hervor, die aussagen, dass ein Dreieck unter gewissen Bedingungen der Gestalt nach 
gegeben ist. Nach Satz 80 dieses Buches ist das der Fall bei einem Dreieck, von dem ein Winkel und das Verhältnis zwischen dem Rechteck aus den einschliessenden Seiten und dem Quadrat der gegenüberliegenden Seite gegeben sind. Die übrigen Winkel des Dreiecks und die Verhältnisse zwischen seinen Seiten werden also durch diese Grössen bestimmt. Im übrigen enthalten die Data weitergehende Sätze derselben Art.

Für numerische Berechnung lassen solche Sätze sich jedoch erst dann benutzen, wenn unter der einen oder anderen Form der Zusammenhang zwischen einem in Winkelmaass gegebenen Winkel und dem Verhältnis von Strecken bestimmt ist. Einen derartigen Zusammenhang kannte man wohl für die wenigen Centriwinkel regulärer Polygone, deren Seiten man konstruieren und dadurch berechnen konnte; aber teils scheint man diese Berechnung lange unterlassen zu haben, teils war hier nur die Rede von ganz einzelnen Winkeln.

Hieraus lässt sich schliessen, dass die Anwendung der Mathematik auf die Astronomie, die mit Eudoxus ihren Anfang genommen hatte, zu Euklids Zeiten noch nicht zu sonderlich genauen Bestimmungen geführt haben kann. Denn das, was man einigermassen genau beobachten kann, sind eben Winkel, und diese verstanden die Mathematiker nicht zu gebrauchen, weshalb man dann wieder umgekehrt auf derartige Bestimmungen keinen Fleiss verwandte. Man hat sicher in der Schule des Eudoxus und namentlich in derjenigen Platos seine Gleichgültigkeit in dieser Beziehung auf dieselbe Weise beschönigt wie die Gleichgültigkeit gegenüber der aus. führlichen Berechnung von irrationellen Grössen, und gemeint, dass man sich, wenn sich bei empirischen Bestimmungen doch keine mathematische Genauigkeit erreichen liesse, ebensogut mit einer gröberen Bestimmung 
begnügen könne. Wenn man solche als Postulate aufstellte, so kam es nur darauf an mit absoluter Sicherheit diejenigen Resultate abzuleiten, die aus diesen einmal aufgestellten Voraussetzungen folgen.

Wurde man nun auch auf diese Weise dahin gebracht das Messen von Winkeln zu vernachlässigen, so mussten dennoch Winkelgrössen sich bei den astronomischen Bestimmungen von selbst geltend machen. Beispielsweise konnten sie sich darbieten als Verhältnisse zwischen den Zeiten, in denen ein Bogen und der ganze Kreis bei einer gleichförmigen Kreisbewegung durchlaufen werden. Ein Beispiel für die Art und Weise, wie man bei richtigen mathematischen Schlüssen diese Art von Bestimmungen anzuwenden verstand - und zugleich ein Beispiel für die Ungenauigkeit der damaligen Bestimmung von Winkeln - besitzen wir in einer überlieferten Untersuchung des Aristarch von Samos über Abstand und Grösse der Sonne und des Mondes. Bei dieser Untersuchung, bei der Aristarch jedoch Vorgänger gehabt hat, namentlich den Eudoxus, wird teils der Radius des Erdschattens in der Entfernung des Mondes von der Erde benutzt, dessen Verhältnis zum Radius des Mondes aus der Dauer der Verfinsterung berechnet wird, teils der Winkelabstand zwischen Sonne und Mond in dem Augenblick, wo der Mond sich genau halb erleuchtet zeigt. Während im übrigen der Proportionslehre gemäss mit Verhältnissen zwischen Entfernungen und Radien operiert wird, wird dieser letzte Winkel in Winkelmaass gefunden. Sein Komplement wird von Aristarch auf $3^{0}$ veranschlagt, und daraus wird abgeleitet, dass der Abstand der Sonne 19 mal so gross ist wie der des Mondes, was dasselbe ist als wenn man annimmt, dass in unserer trigonometrischen Sprache $\sin 3^{0}=\frac{1}{19}$. 
Um dahin zu gelangen, benutzt Aristarch einen Hülfssatz, der sich trigonometrisch folgendermassen ausdrücken lässt: Wenn der Winkel $\vartheta$ von 0 bis $\frac{\pi}{2}$ wächst, so wird das Verhältnis $\frac{\vartheta}{\sin \vartheta}$ wachsen und $\frac{\vartheta}{\operatorname{tg} \vartheta}$ abnehmen. Diesen Satz betrachtet er als bekannt, und wir erkennen auch leicht die Verbindung zwischen ihm und älteren Untersuchungen, deren Bedeutung uns dadurch klarer wird. $\frac{\vartheta}{\sin \vartheta}$ und $\frac{\vartheta}{\operatorname{tg} \vartheta}$ sind nämlich dem Radiusvector und der Abscisse der Quadratrix (vergl. S. 77) proportional, und die angeführten Resultate haben sich dann natürlich an die Untersuchung dieser Kurve angeschlossen.

Zugleich wusste man aus der Bestimmung der Seiten regulärer Polygone, dass $\sin \frac{\pi}{6}=\frac{1}{2}$ und $\operatorname{tg} \frac{\pi}{8}=\frac{1}{\sqrt{2}+1}$, oder, da $\sqrt{2}>\frac{7}{5}$, dass $\operatorname{tg} \frac{\pi}{8}<\frac{5}{12}$. Hieraus erhält man zur Bestimmung von $\sin 3^{0}$ oder $\sin \frac{\pi}{60}$

$$
\sin \frac{\pi}{60}>\frac{1}{10} \cdot \sin \frac{\pi}{6}>\frac{1}{20}
$$

und

$$
\sin \frac{\pi}{60}<\operatorname{tg} \frac{\pi}{60}<\frac{2}{15} \cdot \frac{5}{12}<\frac{1}{18}
$$

mithin näherungsweise $\sin 3^{0}=\frac{1}{19}$.

Es ist beachtenswert, dass sich dasselbe Verfahren, da $\sin \vartheta<\vartheta<\operatorname{tg} \vartheta$, oder wie die Griechen es ausdrücken, da ein einbeschriebenes Polygon einen kleineren, ein umbeschriebenes einen grösseren Umfang hat als der Kreis, auf eine genährte Bestimmung von $\pi$ anwenden lässt. Danach ergiebt sich

$$
3<\pi<3 \frac{1}{3} .
$$

Bestimmungen dieser Art konnte man seit den Zeiten 
Antiphons und Brysons (S. 69, 70) ausführen, denn da man die von diesen begangenen Fehler erkannte, war man auch imstande sie zu vermeiden. Die geometrischen Hülfsmittel für die Erreichung einer grösseren Genauigkeit, die in der Berechnung der Umfänge von regulären Polygonen mit mehr Seiten bestehen würden, besass man auch zu den Zeiten Euklids. In dieser Beziehung dürfen wir nämlich nicht nur an Polygonseiten denken, deren Irrationalität er ausdrücklich darstellt, denn dabei beschränkt er sich auf diejenigen, die bei der Konstruktion regulärer Polyeder, benutzt werden, und er teilt nicht alles mit, was man, nach seinem Buche und z. B. nach Aristarchs Benutzung der Seite des regelmässigen umbeschriebenen Achtecks $\left(2 \operatorname{tg} \frac{\pi}{8}\right)$ zu urteilen, damals vermochte. Um jedoch wirklich die vorliegenden geometrischen Hülfsmittel für eine genauere Bestimmung von $\pi$, oder für eine genauere Anwendung gemessener Winkel zu benutzen, musste sich einmal das Bedürfnis nach solchen zeigen, und zweitens war grosse Energie erforderlich um damals eine Berechnung durchzuführen, bei der verschiedene Quadratwurzeln auszuziehen waren. Unter anderem machte sich dies Bedürfnis geltend bei der, im Vergleich mit früheren Messungen, genaueren Bestimmung der Schiefe der Ekliptik durch Eratosthenes und bei seiner Gradmessung. Um bei diesen den Unterschied in der Polhöhe und die Entfernung von zwei Orten mit nahezu gleicher Länge für die Berechnung des Erddurchmessers zu benutzen, musste man eine leidlich gute Bestimmung von $\pi$ besitzen. Archimedes war es, der in seiner Kreismessung die Schwierigkeiten überwandt, die sich einer solchen entgegen stellten. Wir wollen hier kurz über den Inhalt seiner Schrift berichten, in der jedoch leider keine Aufklärung darüber enthalten ist, wie 
er die grösste der vorhandenen Schwierigkeiten überwand, nämlich die Bestimmung der Quadratwurzeln.

Archimedes beginnt damit mittels des Exhaustionsbeweises darzuthun, dass der Kreis denselben Inhalt hat wie ein Dreieck, das die Peripherie zur Grundlinie und den Radius zur Höhe hat. Dadurch wird die Quadratur des Kreises auf die Berechnung der Kreisperipherie zurückgeführt. Archimedes beweist, dass das Verhältnis dieser Peripherie zum Durchmesser, also die Zahl, die in neuerer Zeit $\pi$ genannt worden ist, kleiner als $3 \frac{1}{7}$, aber grösser als $3 \frac{1}{7} \frac{0}{1}$ ist. Das wird dadurch bewiesen, dass selbst der Umfang des einbeschriebenen 96ecks grösser ist als $3 \frac{1}{7} \frac{0}{1} d$, und selbst der Umfang des umbeschriebenen 96 ecks kleiner ist als $3 \frac{1}{7} d$, wenn wir mit $d$ den Durchmesser des Kreises bezeichnen.

$\mathrm{Zu}$ diesem Ergebnis gelangt Archimedes dadurch, dass er aus den Verhältnissen zwischen den Seiten eines rechtwinkeligen Dreiecks mit einem gewissen Winkel $\mathrm{x}$ die Verhältnisse zwischen den Seiten eines rechtwinkeligen Dreiecks mit dem halben Winkel $\left(\frac{1}{2} \mathrm{x}\right)$ bestimmt. Wenn er die obere Grenze für die Peripherie sucht, so lässt er die Dreiecke mit den Winkeln $\mathrm{x}$ und $\frac{1}{2} \mathrm{x}$ die diesen Winkeln anliegende Kathete gemeinsam haben, und wenn er die untere Grenze sucht, die Hypotenuse; in beiden Fällen aber lässt die gefundene Relation zwischen den Verhältnissen sich in der trigonometrischen Sprache unserer Zeit wiedergeben durch

$$
\operatorname{tg} \frac{1}{2} x=\frac{\sin x}{1+\cos x}\left(\operatorname{oder} \frac{\operatorname{tg} x}{\sec x+1}\right)
$$

Diese Übereinstimmung wird bei der Anwendung jedoch durch den Umstand verdeckt, dass bei der einen Untersuchung obere Grenzen für die Quadratwurzeln gebraucht werden, zu denen der Übergang zwischen den 
Verhältnissen verschiedener Seiten desselben rechtwinkeligen Dreiecks $\left(\sin ^{2} x+\cos ^{2} x=1\right)$ Veranlassung giebt, und bei der anderen untere Grenzen, und dass diese durch verschieden gebildete Näherungswerte ausgedrückt werden.

Indem A r c h i m ed es von einem rechtwinkeligen Dreieck mit dem Winkel $\frac{\pi}{6}$ ausgeht und wiederholt zu neuen Dreiecken übergeht, findet er, dass (mit unseren trigonometrischen Bezeichnungen für die untersuchten Verhältnisse)

$$
\operatorname{tg} \frac{\pi}{96}<\frac{153}{4673 \frac{1}{2}} \text { und } \sin \frac{\pi}{96}>\frac{66}{2017 \frac{1}{4}}
$$

und gelangt dadurch $\mathrm{zu}$ den oben angegebenen Grenzen für $\pi$.

Nachdem durch Archimedes' Arbeit das Eis einmal gebrochen war, soll Apollonius eine noch genauere Berechnung geliefert haben. Vielleicht rührt von ihm der Wert 3,1416 her, der im wesentlichen der Genauigkeit entspricht, die sich später in den Sehnentafeln des Ptolemäus findet, und dem wir später bei indischen Schriftstellern begegnen werden.

Archimedes' Schrift enthält faktisch Bestimmungen der unteren Grenzen für $\sin \frac{\pi}{n}$ und der oberen Grenzen für $\operatorname{tg} \frac{\pi}{n}$ für $n=6,12,24,48,96$. Die letzte Bestimmung liefert auch eine brauchbare obere Grenze für $\frac{\pi}{96}$, und Aristarchs Arbeit zeigt, dass man imstande war sie zu benutzen. Apollonius' Bestimmung von $\pi$ muss zu einer noch genaueren Bestimmung des sinus eines kleinen Bogens oder derjenigen Grösse geführt haben, über deren Werte wir Tabellen von späteren griechischen Astronomen besitzen, nämlich der Sehne des 
doppelten Bogens. Um eine vollständige Tafel der Sehnen $\mathrm{zu}$ Vielfachen dieses kleinen Bogens zu berechnen, ist nun weiter nichts erforderlich als die Kenntnis des Satzes über einbeschriebene Vierecke, der jetzt der ptolemäische genannt wird, weil Ptolemäus ihn ausdrücklich für die Berechnung seiner Sehnentafel anwendet, oder die Kenntnis eines anderen Satzes, der sich auf ähnliche Weise anwenden lässt. Einen solchen hat man zu und nach der Zeit des Archimedes und Apollonius leicht finden können in dem Moment, wo man wirklich eine Sehnentafel wünschte, und die grösste Schwierigkeit hat auch hier darin bestanden, die zur Durchführung der Arbeit notwendigen Quadratwurzeln zu berechnen; aber gerade dadurch wurde man gezwungen, die dazu dienenden Methoden zu verbessern. Wie früher angeführt (S. 62) war dieser Fortschritt mit der Einführung der Sexagesimalbrüche verbunden.

Die erste Sehnentafel, von der wir sichere Nachricht haben, stammt aus dem zweiten Jahrhundert v. Chr. und ist von dem grossen Astronomen Hipparch verfasst. Diese ist, ebenso wie eine spätere von Menelaus, verloren gegangen. Die Sehnentafel, die sich in Ptolemäus' Almagest findet, ist uns dagegen überliefert und hat, da sie auf den älteren hat aufgebaut werden können, die grösste Volständigkeit und Genauigkeit erhalten. Sie geht mit Intervallen von $\frac{1}{2}^{\circ}$ bis $\mathrm{zu}$ einem Bogen von $180^{\circ}$. $\mathrm{Da}$ der sinus die Hälfte von der Sehne des doppelten Bogens ist, so spielt diese Tafel dieselbe Rolle wie eine Tafel der sinus von solchen Bogen, die mit Intervallen von $\frac{1}{4}^{0}$ bis zu $90^{\circ}$ hinaufreichen. Der Durchmesser des Kreises wird gleich 120 gesetzt, und die Sehnen werden nach dem Sexagesimalsystem in Ganzen, Minuten und Sekunden ausgedrückt, das heisst, wie bei der Winkelteilung, in Brüchen mit den Nennern 60 und $60^{2}$; das 
Verhältnis der Sehnen zum Durchmesser wird also in Brüchen mit dem Nenner 432000 angegeben. Zur Benutzung bei der Interpolation werden Dreissigstel der Differenzen zwischen den aufeinander folgenden Sehnen, den Bogendifferenzen von 1 Minute entsprechend, hinzugefügt.

Für die Berechnung dieser Tafel benutzt Ptolemäus hauptsächlich den Satz über das einbeschriebene Viereck. Dieser lässt sich unmittelbar benutzen, um die Sehne zu der Summe oder Differenz zweier Bogen, und dadurch anch die Sehne zu dem verdoppelten und halbierten Bogen zu berechnen. Wenn man von den bekannten Sehnen ausgeht, so kann man auf diesem Wege dahin gelangen, Sehnen zu Bogen von $1 \frac{1}{2}^{0}$ und $\frac{3}{4}^{0} \mathrm{zu}$ berechnen. Aus diesen wird die Sehne zu $1^{0}$ durch eine Art von Interpolation berechnet, die darauf beruht, dass das Verhältnis zwischen Sehne und Bogen abnimmt, wenn der Bogen wächst, dass also

$$
\frac{\text { Sehne } \frac{3}{4}^{0}}{\frac{3}{4}}>\frac{\text { Sehne } 1^{0}}{1}>\frac{\text { Sehne } 1 \frac{1}{2}^{0}}{1 \frac{1}{2}}
$$

Für den hier benutzten geometrischen Satz, den auch Aristarch anwandte, teilt Ptolemäus einen hübschen geometrischen Beweis mit. Nachdem die Sehne zu $1^{0}$ auf diese Art gefunden ist, wird der ptolemäische Satz für die successive Berechnung der übrigen Sehnen benutzt.

Da eine Sehnentafel dieselbe Rolle spielt wie eine Sinustafel, so kann man, wenn man will, mit Hülfe dieser Tafel und des pythagoreischen Lehrsatzes jedes Stück eines ebenen rechtwinkeligen Dreiecks durch zwei andere, unter denen sich eine Seite befindet, bestimmen und dadurch, wenn auch durch beschwerliche Rechnungen, die Bestimmungen ausführen, die in die ebene Trigonometrie hineingehören. Im Almagest finden sich Beispiele 
für verschiedene solcher Bestimmungen. Den Astronomen lag jedoch sehr viel daran, auch die sphärisch-trigonometrischen Bestimmungen zu gewinnen. Hierzu war vor allen Dingen etwas sphärische Geometrie erforderlich.

\section{Sphärische Geometrie.}

Aus der Geometrie der Kugel haben wir bei Euklid nur den Satz über das Verhältnis zwischen den Inhalten von Kugeln gefunden, zu dem Archimedes die exakte Bestimmung von Oberfläche und Inhalt hinzufügte; aber dabei konnten die Astronomen nicht stehen bleiben. Namentlich mussten sie Mittel haben um die Lage von Punkten auf der Kugel, von Sternen am Himmel, von Orten auf der Erde zu charakterisieren. Das geschieht durch Beziehung auf einen grössten Kreis, der auf die eine oder andere Weise als bekannt betrachtet werden darf, auf Horizont, Äquator oder Ekliptik am Himmel, Äquator auf der Erde, und diese Beziehung konnte nicht wohl von der verschieden sein, die jetzt durch die gewöhnlichen sphärischen Koordinaten ausgeführt wird. Eine solche Beziehung liegt zu Grunde, wenn Eratosthenes, um die Grösse der Erde zu bestimmen, die Entfernung zwischen zwei Orten von derselben Länge und bekanntem Breitenunterschiede misst, selbst wenn die Einführung der Bezeichnungen Länge und Breite erst dem $\mathrm{H}$ ipparch zugeschrieben wird. Im übrigen wollen wir daran erinnern, dass diejenige Einteilung der Kugel, die Euklid im 12ten Buche der Elemente anwendet (S. 171), genau einer Einteilung durch solche Koordinaten entspricht.

Eine mehr oder weniger direkte Anwendung sphärischer Koordinaten lässt sich benutzen um die Punkte des Himmels oder der Erde auf einer gedrechselten Kugel 
ahzubilden. Das erstere ist jedenfalls geschehen. Indessen waren die Griechen durch ihre hoch entwickelte Geometrie in den Stand gesetzt die schwierigeren Aufgaben zu lösen, die sich darbieten, wenn man auf zweckmässige Weise eine Kugel auf einer Ebene abbilden will. Mit Rücksicht hierauf wird es in dieser Darstellung der Geschichte der Mathematik genügen anzuführen, dass die Griechen verschiedene Anwendungen der in geometrischer Beziehung wichtigen und interessanten stereographischen Projektion gemacht haben. Diese besteht wie bekannt in einer Centralprojektion der Kugelfläche von einem auf ihr gelegenen festen Punkt auf denjenigen grössten Kreis, der den festen Punkt zhm Pol hat, und zeichnet sich dadurch aus, dass jeder Kreis auf der Kugel als Kreis auf die Ebene projiciert wird, und dass Winkel ibre Grösse behalten.

Aus den vorliegenden Anwendungen weiss man, dass die Griechen wenigstens die erste von diesen Eigenschaften gekannt haben, die in naher Verbindung steht mit der Lehre von den beiden Systemen von Kreisschnitten an einem schiefen Kegel. Eine Bestimmung von diesen findet sich bei Archimedes, und die Lehre von den verschiedenen Schnitten an demselben Kegel wird von Apollonius weiter entwickelt. Die stereographische Projektion darf also wohl als eine Frucht der Lehren dieser Männer betrachtet werden. Sie fand zu den Zeiten Hipparchs Anwendung an einem Apparat, der gebraucht wurde, um aus der augenblicklichen Höhe eines bekannten Sterns die Zeit in der Nacht $z u$ bestimmen. Dazu benutzte man zwei Scheiben, von denen die eine Projektionen bekannter Sterne enthielt, die andere Projektionen des Horizonts und parallel $\mathrm{zu}$ ihm gelegter Schnittkreise, beide Teile vom Südpol des Himmels aus projiciert. Es kam darauf an, die eine Scheibe so um das Bild des 
Nordpols zu drehen, dass der beobachtete Stern auf dem seiner Höhe entsprechenden Schnittkreise zu liegen kam. Eine andere Anwendung findet sich in der Geographie des Ptolemäus.

Die griechische Geometrie lieferte jedoch nicht nur die Mittel, um astronomische Bestimmungen durch solche mechanische Operationen vorzunehmen. Wir sahen soeben, dass man seit den Zeiten der grossen Geometer begonnen hatte sich mit den tabellarischen Arbeiten zu beschäftigen, die notwendig waren um derartige Aufgaben durch Berechnung zu lösen. Gleichzeitig muss die Geometrie die sphärisch-geometrischen Sätze hervorgebracht haben, auf denen die Anwendung der Tabellen beruht, und die in anderer Form unseren sphärisch-trigonometrischen Formeln über das rechtwinkelige Dreieck entsprechen. Diese Form lernen wir bei Ptolemäus kennen. Er benutzt dafür einen Satz, den man nach seiner Angabe den Satz des Menelaus genannt hat. In seiner planimetrischen Gestalt heisst dieser Satz: Wenn eine Transversale die den Ecken $A, B$ und $C$ eines Dreiecks $A B C$ gegenüberliegenden Seiten in $D, E$ und $F$ schneidet, so ist

$$
\frac{B D}{C D} \cdot \frac{C E}{A E} \cdot \frac{A F}{B F}=1
$$

der Satz bleibt aber auch bestehen, wenn man die geraden Linien mit grössten Kreisen auf derselben Kugel vertauscht, die Strecken mit den sinus der Bogenstrecken, oder, wie Ptolemäus sagen muss, mit den Sehnen des doppelten Bogens. Wahrscheinlich ist der Satz in beiden Gestalten weit älter als Menelaus. Die planimetrische gehört durchaus unter die Gegenstände, die in Euklids Porismen behandelt wurden, und was den sphärisch-geo metrischen Satz angeht, so verstand wenigstens Hipparch 
die Berechnungen auszuführen, zu denen er bei Ptolemäus benutzt wird.

Diese Berechnungen sind dieselben, die in der sphärischen Trigonometrie ausgeführt werden durch die 4 Relationen, die in einem rechtwinkeligen sphärischen Dreieck zwischen den 3 Seiten oder zwischen 2 Seiten und einem Winkel stattfinden. $A B C$ sei ein solches Dreieck mit

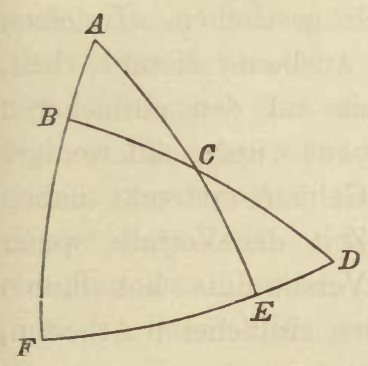
dem rechten Winkel bei $B$, und $D, E$ und $F$ seien die Punkte, in denen der grösste Kreis mit dem Pol $A$ die Seiten des sphärischen Dreiecks schneidet. Dann ist in Gradmaass $\overparen{F E}=\angle A$, und $\overparen{E D}=90^{\circ}-\angle A$. Die übrigen Bogen der Figur sind Seiten des Dreiecks $A B C$ oder deren Komplemente. Wenn man nun nach einander die vier Dreiecke $A B C$, $C D E, A F E$ und $D B F$ und jedesmal den vierten grössten Kreis als Transversale betrachtet, so erhält man eben die erwähnten vier Relationen. Erst bei späten arabischen Schriftstellern finden wir eine Relation zwischen 2 Winkeln und eine Seite.

\section{Verfall der griechischen Geometrie.}

Die Entwickelung der berechnenden Geometrie bei den Griechen, die wir soeben verfolgt haben, reichte nicht ganz wenig über den Zeitpunkt hinaus, an dem die übrige griechische Geometrie ihren Höhepunkt erreicht hatte. In den mehr abstrakten, geometrischen und in geometrischer Form dargestellten rein mathematischen Untersuchungen, in denen die Griechen besonders weit gelangten, 
können wir nämlich eigentlich nach Apollonius keinen Fortschritt von grösserer Bedeutung nachweisen. Das ist indessen nicht so $\mathrm{zu}$ verstehen, als ob die mathematische Arbeit sofort nach ihm aufgehört hätte. Der gelegte Grund war so sicher, und die angewandten Methoden der Behandlung so fruchtbar, dass es den Schülern der grossen Mathematiker, so lange die Zeitverhältnisse es zuliessen, leicht gewesen sein muss, in den angefangenen Richtungen weiter zu arbeiten. Das ist auch geschehen. Indessen ist es leicht erklärlich, dass die Ausbeute dieser Arbeit, die sich gerade dadurch, dass sie auf den einfacheren Untersuchungen der Meister aufgebaut wurde, auf weniger zugängliche und mehr specielle Gebiete erstreckt haben kann, in der nun kommenden Zeit des Verfalls weder so viel Interesse noch so viel Verständnis hat finden können wie die zu Grunde liegenden einfacheren Arbeiten, und deshalb verloren gegangen ist.

In der That liegen denn auch nur vereinzelte Mitteilungen vor über die geometrischen Arbeiten der Nachfolger oder Zeitgenossen der grossen Geometer. Von Nikomedes und seiner Konchoide haben wir bereits gesprochen (S. 82). Von Perseus wird berichtet, dass er die sogenannten spirischen Kurven untersucht habe, die, wie man annimmt, Schnitte an der Fläche waren, die durch Umdrehung eines Kreises um eine in seiner Ebene liegende Axe erzeugt wird (Wulst). Eine einzelne von diesen Kurven ist vielleicht früher von Eudoxus untersucht worden, der zur Darstellung der scheinbaren Bahnen der Planeten und ihrer Knotenpunkte eine Kurve, Hippopede genannt, angewandt hat, die möglicherweise dieselbe gewesen ist, die wir jetzt Lemniskate nennen. Die Cissoide des Diokles ist auch in unseren Tagen bekannt und wird als nützliches Beispiel für die Anwendung der Differential- und Integralrechnung auf die Geometrie 
benutzt. Diokles verdankt man auch eine neue Lösung von Archimdes' kubischer Gleichung mit Hülfe der Kegelschnitte (vergl. S. 216).

Aus der nächsten Zeit nach den grossen Geometern stammen sicherlich auch die wichtigsten der von Pappus angeführten Resultate, die sich nicht auf diese selbst zurückführen lassen. Einige können auch in den einzelnen Perioden entstanden sein, in denen das Verständnis wieder aufleuchtete, und ein einzelnes legt Pappus sich selbst bei. Wir wollen hier einige von diesen Resultaten anführen. Ausser der von Archimedes gefundenen ebenen Spiralenfläche (S. 183) hat man Flächen bestimmt, begrenzt von Spiralen, die auf entsprechende Weise auf der Kugel dargestellt werden. Archimedes' Bestimmung des Inhaltes der Kugeloberfläche wurde dabei zu Grunde gelegt. - Die Projektion eines ebenen Schnittes durch eine Erzeugende einer windschiefen Schraubenfläche (deren Erzeugenden der Grundfläche parallel sind) auf die Grundfläche ist ein Quadratrix. - Pappus legt sich selbst den wichtigen allgemeinen Satz bei, dass das Volumen eines Umdrehungskörpers gleich ist dem Produkte aus der Fläche, die von der Meridiankurve umschlossen wird, und dem Wege, den der Schwerpunkt dieser Fläche während der Umdrehung durchläuft. Dieser Satz, der nach einem späteren Wiederentdecker den Namen der Guldinschen Regel erhalten hat, muss im übrigen bei der von den Alten benutzten geometrischen Darstellung der für Schwerpunktsbestimmungen nötigen Integrationen sehr nahe gelegen haben. Pappus' allgemeine Aufstellung desselben ist jedoch ein wirkliches Verdienst. - Bei Pappus findet sich ferner eine Erweiterung des Ortes zu vier Geraden (vergl. S. 213). Für die Bestimmung einer gewissen Kurve stellt er die Forderung auf, dass das Verhältnis zwischen den Produkten der Abstände eines 
Kurvenpunktes von zwei Gruppen von in beliebiger Anzahl gegebenen Geraden einen gegebenen Wert haben soll. Das Verhältnis wird in Übereinstimmung mit Euklids fünftem Buche als ein zusammengesetztes Verhältnis dargestellt (vergl. S. 145). Pappus teilt jedoch keine Eigenschaften der Kurve mit, die über die Definition hinausgingen. Indessen ist die Kurve ein wichtiger Ausgangspunkt für Descartes' analytische Geometrie geworden.

Wie interessant auch mehrere der hier angeführten Resultate sein mögen, so spricht doch der Umstand, dass sich bei Pappus neben den zahlreichen und bedeutungsvollen Mitteilungen über die Arbeiten der alten Mathematiker, die wir bereits bei unserer Erwähnung von diesen in reichem Maasse benutzt haben, keine anderen sonderlich neuen Sätze nachweisen lassen, dafür, dass der Zeitraum zwischen Apollonius und Pappus innerhalb der Geometrie keinen Fortschritt von bleibender Bedeutung hervorgebracht hat. Dass diese 500 Jahre dann einen bedeutenden Rückgang in der Kenntnis und dem Verständnis der mathematischen Schätze früherer Zeiten mit sich gebracht haben, war zu erwarten. Das erkennt man auch deutlich aus den vielen Hülfssätzen zu den alten Schriften, die Pappus aus dieser Zwischenzeit aufbewahrt hat. Durch die sorgfältige, oft kleinliche Erklärung, die diese von Einzelheiten geben, tritt es nämlich deutlich hervor, wie wenig man sich den Blick für die Bedeutung der Gesamtheit bewahrt hatte.

Wenn man nun fragt, wie ein solcher Niedergang bei einer so gründlich und reich entwickelten Wissenschaft, wie die griechische es war, möglich sein konnte, so lässt sich wohl auf die in der historischen Übersicht genannten verschiedenen äusseren Umstände hínweisen, aber diese 
geben keine genügende Erklärung. Da, wie wir bei Pappus sehen, manche ältere Schriften beständig erhalten blieben, so könnte man erwarten, dass sich durch diese die verlorene mathematische Einsicht hätte wiedergewinnen und die unterbrochene Entwickelung hätte wiederaufnehmen lassen. Doch geschah das nicht im Altertum, und bei den Arabern und demnächst bei den Europäern der neueren Zeit musste das Studium der Schriften der Alten von einer ganz neuen Entwickelung - die zugleich nach anderen Richtungen hin fruchtbar war - begleitet werden, bevor man sich das, was diese Schriften enthielten, vollkommen aneignete.

Der Grund für einen solchen Niedergang und für das Fehlen der Bedingungen für einen Wiederaufbau ist in den Mängeln zu suchen, die in den aus dem Altertum überlieferten Schriften selbst enthalten sind. Auf diese Mängel haben wir gelegentlich schon hingewiesen, aber wir wollen sie noch einmal zusammenstellen.

Ein Mangel hängt genau mit etwas zusammen, was in anderer Beziehung unsere höchste Bewunderung hervorgerufen hat, nämlich mit der ausserordentlichen Sorgfalt, mit der man sich durch bestimmte Formen der logischen Unanfechtbarkeit versicherte. Wegen dieser wurde nämlich alles bei Seite gesetzt, was die Zugänglichkeit erleichtern, einen Überblick verschaffen oder die Absichten bei den einzelnen Operationen verdeutlichen konnte. Allerdings erhielt sich bis zu einem gewissen Grade lange das Verständnis für das, was durch diese Formen erreicht wurde, und zum Teil lernte man sie nachzuahmen; aber indem man seine ganze Kraft hierauf verwandte und keine Anleitung zu einem allgemeineren Verständnis des ganzen Zusammenhanges erhielt, blieb man bei dem Interesse für diese Formen und bei einer sparsamen Aneignung der einfachsten von den Eigenschaften stehen, die nun 
einmal in sie hineingelegt waren. Da diese Einzelheiten in den bewunderten Formen auftraten, so erhielten sie ein solches Gepräge von Vollkommenheit, dass man den Mut zu selbständigen Arbeiten verlieren musste. Die Ausbeute aus solchen würde nämlich, jedenfalls anfänglich, in einer sehr viel weniger vollkommenen Form aufgetreten sein.

Mit der geometrischen Form, die die Algebra und die allgemeine Grössenlehre angenommen hatten, war auch ein Übelstand verbunden. Sicherlich gab diese geometrische Form an Übersichtlichkeit und Verwendbarkeit für wirkliche Ausführung von algebraischen Operationen der jetzigen algebraischen Zeichensprache nicht so viel nach, wie ein moderner Leser anzunehmen geneigt sein wird. Derjenige, welcher mit dieser Art der Darstellung vertraut ist, also die Bedeutung der Figuren kennt, kann das Umlegen von ihnen und das Operieren mit ihnen mit derselben Leichtigkeit vornehmen, mit der man jetzt Buchstabenausdrücke versetzt und zusammenzieht, und ferner kann er beim mündlichen Unterricht dadurch, dass er auf die Figuren zeigt, die vorzunehmenden Operationen seinen Schülern verständlich machen. Das trug dazu bei, dass ein vollkommenes Verständnis so lange aufrecht erhalten werden konnte, als sich der mündliche Unterricht in Alexandria in Ruhe fortsetzen liess. Sobald aber diese Ruhe gestört wurde und die durch mündlichen Unterricht aufrecht erhaltene Tradition verloren ging, man also allein auf das Studium der sorgfältig ausgearbeiteten Werke angewiesen war, musste ein empfindlicher Rückgang eintreten. Die geometrische Darstellung der Algebra ist nämlich nicht leicht $\mathrm{zu}$ lesen. Das wird verständlich, wenn man festhält, dass Text und Figur bis zu einem gewissen Grade jeder für sich auftreten müssen, 
man also während des Studiums von dem einen zum anderen hin und her fahren muss.

$\mathrm{Zu}$ diesen formellen Schwächen kam nun noch eine andere, die wir schon oft genannt haben und die im wesentlichen den Inhalt selbst betraf. Die griechischen Mathematiker hatten ihre wissenschaftliche Würde so hoch gehalten, dass sie aus ihren Hauptwerken alles ausschlossen, was ihnen nicht vollkommen exakt erschien. Dadurch wurden, wie wir namentlich gesehen haben, als wir über das Ausziehen der Quadratwurzel sprachen, wirkliche numerische Berechnungen, die in der Regel nur eine Annäherung geben konnten, ausgeschlossen und der weniger hoch geachteten Logistik zugewiesen. Damit liess man zugleich die praktischen Anwendungen fahren, die den Mathematikern hätten neue Impulse geben und das Interesse für die Wissenschaft in den Zeiten hätten wachhalten können, wo der Blick für den Wert der Wissenschaft selbst nicht mehr vorhanden war.

Die schädlichen Folgen dieser Vernachlässigung der Anwendungen der Mathematik treten vielleicht am deutlichsten hervor, wenn man ihnen die nützlichen Folgen gegenüberstellt, die sich dadurch ergaben, dass man sich nach einzelnen Richtungen hin solcher Vernachlässigung nicht schuldig machte. Wir haben soeben gesehen, dass die Anwendungen auf die Astronomie die Entwickelung der berechnenden Geometrie fortdauern liessen, nachdem im übrigen ein Stillstand eingetreten war. Zugleich sahen wir, dass man in Verbindung hiermit fortfuhr bedeutende Fortschritte in der Ausführung praktischer Berechnungen zu machen. Dadurch entstand eine Vertrautheit mit den Zahlen, die sicherlich dazu beigetragen hat, die Arithmetik über den von den älteren griechischen Mathernatikern erreichten Standpunkt so weit hinaus zu entwickeln, wie sie uns bei Diophant entgegentritt. 


\section{Die spätere griechische Arithmetik; Diophant.}

Die allgemeine wissenschaftliche Grundlage für die griechische Arithmetik haben wir aus dem 7ten-9ten Buche Euklids kennen gelernt. Wenn nun auch diese Grundlage nicht den Umfang und die wissenschaftliche Sicherheit besitzt wie der in den übrigen Büchern gelegte Grund für die Geometrie und die in geometrischer Form gegebene allgemeine Grössenlehre, so ist dennoch dieselbe allgemeine Form beibehalten. Obgleich die Rede von Zahlen ist, werden die Sätze nicht durch Zahlenbeispiele verdeutlicht. Ohne die Behandlung vieler solcher Zahlenbeispiele kann die allgemeine Theorie jedoch nicht ent-standen sein. So haben die Pythagoreer sicherlich bereits viele Beispiele für die sogenannten vollkommenen Zahlen gekannt. Über verschiedene andere Zahlenformen, namentlich die Polygonalzahlen, mit denen man sich frühzeitig beschäftigte, haben wir bereits gesprochen, als wir die geometrische Arithmetik schilderten. Derartige Untersuchungen sind sicherlich in früheren Zeiten mit der praktischen Ausrechnung solcher Zahlen verbunden gewesen. Endlich haben wir gesehen, dass eine Klasse von zahlentheoretischen Untersuchungen frühzeitig die Aufmerksamkeit auf sich zog, nämlich solche, die die Anwendung der allgemeinen Lösungen der Gleichungen zweiten Grades auf numerische Gleichungen betrafen. Man untersuchte die Bedingungen dafür, dass Zusammensetzungen von Zahlen zu rationalen Aufösungen der quadratischen Gleichungen führten, also die Bedingungen dafür, dass gewisse Zahlengebilde Quadrate werden. Das muss in der Regel bei der Behandlung solcher Gleichungen geschehen, die wir jetzt unbestimmte Gleichungen zweiten Grades nennen. Wir haben auch gesehen, dass diese 
sich bei der genäherten Ausziehung der Quadratwurzel aus einzelnen bestimmten Zahlen, wie 2, benutzen lassen. Indessen haben wir in der älteren griechischen Mathematik doch nur einzelne Beispiele für solche unbestimmte Gleichungen gefunden; wir heben sie aber hier hervor als den Anfang einer Richtung, in der es die Griechen, wie wir bald sehen werden, später sehr viel weiter brachten. Das Interesse, das zuerst durch den Wunsch nach rationalen Lösungen geweckt worden war, hat sich dann später den unbestimmten Gleichungen selbst zugewendet.

Ein Beispiel dafür, dass man auch während der alexandrinischen Zeit praktische Untersuchungen über gewisse Klassen von ganzen Zahlen fortgesetzt hat, liegt in dem Berichte darüber, wie Eratosthenes die ersten Primzahlen aufgezählt hat mit Hülfe der Methode, die man «das Sieb des Eratosthenes» (cribrum Eratosthenis) nennt. Man schreibt zuerst die ganze Zahlenreihe so weit auf, als man in seiner Untersuchung gehen will, streicht darauf jede zweite Zahl, indem man mit 4 beginnt, jede dritte, indem man mit 6 beginnt, jede fünfte, indem man mit 10 beginnt u. s. w. Die Zahlen, die dabei nicht «ausgesiebt» werden, sind Primzahlen.

Man schreibt dem Archimedes eine verwickelte arithmetische Aufgabe über die Ochsen des Helios zu, aber vielleicht mit Unrecht. Dagegen haben wir ihn im Vorhergehenden gelegentlich eine andere zahlentheoretische Bestimmung ausführen sehen, für die er gerade Verwendung hatte, nämlich die Bestimmung der Summe der ersten Quadratzahlen. Die so gebildeten Summen liefern ein Beispiel für die unter der geometrischen Arithmetik erwähnten Pyramidalzahlen, nämlich für solche, die einer Pyramide mit quadratischer Grundfläche entsprechen. Diese führen zugleich ohne Schwierigkeit zu der Berech. nung der übrigen Pyramidalzahlen. 
Wir sehen also, dass Nikomachus, dem wir namentlich unser Wissen von der Bekanntschaft der Alten mit figurierten Zahlen verdanken, nicht nur mit Bezug auf die Polygonalzahlen, sondern auch auf die Pyramidalzahlen auf dem weiter bauen konnte, was von den Zeiten der grossen Mathematiker her bereits vorlag. Neben der Lehre von diesen Zahlen findet sich bei ihm eine Beobachtung, die eine Fortsetzung des bereits den Pythagoreern bekannten Satzes über die Bildung der Quadratzahlen aus Summen der ersten ungeraden Zahlen darstellt. Diese besteht darin, dass sich auch jede Kubikzahl als Summe von aufeinander folgenden ungeraden Zahlen darstellen lässt. Der in der Formel

$$
n^{3}=\left(n^{2}-n+1\right)+\left(n^{2}-n+3\right)+\ldots+\left(n^{2}+n-1\right)
$$

ausgedrückte allgemeine Satz scheint dem Nikomachus jedoch nicht vollständig bekannt gewesen zu sein.

In Verbindung hiermit sei noch angeführt, dass wir durch einen viel jüngeren römischen Schriftstéller wissen, dass man im Altertum die ersten Kubikzahlen hat summieren können, also gewusst hat, dass

$$
1^{3}+2^{3}+3^{3}+\ldots+n^{3}=\left(\frac{n(n+1)}{2}\right)^{2} .
$$

Dieses Resultat kann aus dem oben genannten abgeleitet worden sein. Bei einem arabischen Schriftsteller findet sich jedoch ein anderer Beweis, der durch seine geometrische Form griechischen Ursprung verrät, und der gleichzeitig zu dem Satze bei Nikomachus und zur Summation der Kubikzahlen geführt haben kann. Dieser lässt sich in der Sprache der jetzigen Algebra folgendermassen wiedergeben: 
29. Die spätere griechische Arithmetik; Diophant.

$$
\begin{gathered}
(1+2+\ldots+n)^{2}-(1+2+\ldots+n-1)^{2}= \\
n \frac{n(n+1)}{2}+n \frac{n(n-1)}{2}=n^{3}
\end{gathered}
$$

zugleich ist aber zu beachten, dass die Differenz zwischen den beiden Quadraten nach gewöhnlicher griechischer Weise als ein Gnomon dargestellt wird, der hier aus den beiden Rechtecken

$$
n(1+2+\ldots+n)=n \frac{n(n+1)}{2}
$$

und

$$
n(1+2+\ldots+n-1)=n \frac{n(n-1)}{2}
$$

besteht.

Bis gegen $300 \mathrm{n}$. Chr. finden sich jedoch nur zerstreute Beiträge $\mathrm{zu}$ einer weiteren Entwickelung der in den besten Zeiten der Geometrie bekannten Arithmetik, und selbst von diesen Beiträgen wissen wir nicht, wie früh das Mitgeteilte bekannt gewesen ist. Erst bei Diophant von Alexandria begegnen wir etwas Neuem von grösserem allgemeinem Interesse. Sein überliefertes arithmetisches Werk zeigt uns Seiten der griechischen Mathematik, von denen wir in den uns erhaltenen Schriften der älteren Mathematiker nur die ersten und undeutlichen Anfänge haben kennen lernen. Allerdings ist die theoretische Grundlage dieses Werkes dieselbe, die wir bei Euklid gefunden haben, und freilich fällt das Ziel von Diophants interessanten Untersuchungen zusammen mit dem früher erwähnten, aus älteren Zeiten stammenden Bestreben irrationale Grössen $\mathrm{zu}$ vermeiden; aber diese Untersuchungen werden von ihm in einem bis dahin unbekannten Umfange vorgenommen. Diese befähigen ihn Beispiele für bestimmte Aufgaben aufzustellen, die unter stark variierenden Formen zu Gleichungen mit rationalen Lösungen führen, und sie bringen ihn namentlich dahin 
zahlreiche unbestimmte Aufgaben zu stellen, bei denen es darauf ankommt rationale Lösungen $\mathrm{zu}$ finden. In seiner ganzen Darstellung findet sich ferner gegenüber den älteren überlieferten Darstellungen der grosse Unterschied, dass er beständig nur specielle Zahlenaufgaben behandelt und nur die zu diesen gehörenden Zahlenoperationen mitteilt, ohne allgemeine Sätze aufzustellen. Da bei ihm nicht nur die gegebenen Zahlen rational sind, sondern auch die gesuchten rational sein sollen, so bedarf er der geometrischen Darstellung keineswegs in demselben Maasse wie sie bei solchen Untersuchungen notwendig ist, deren Resultate auf beliebige Grössen anwendbar sein sollen, einerlei ob diese sich durch Zahlen (d. h. rationale Zahlen) darstellen lassen, oder nicht. Er benutzt wohl die von der geometrischen Darstellung entlehnten Benennungen, wie Rechteck für Produkt, aber dass die behandelten Grössen doch nur Zahlen sind, das geht z. B. daraus hervor, dass die geometrische Homogenität nicht aufrecht erhalten wird; so wird bei ihm ohne weiteres eine Seite $z u$ einer Fläche addiert.

Diophant legt sogar so wenig Gewicht auf formelle Allgemeingültigkeit, dass er durchgehends, wenn in einer Aufgabe im allgemeinen gesagt wird, dass eine gewisse Zahl einen gegebenen Wert haben soll, dieser sofort einen bestimmten Wert beilegt; mit diesem rechnet er dann zunächst, so dass die vorgelegte allgemeine Aufgabe nur insofern gelöst wird, als man aus dem gewählten Beispiel schliessen kann, wie man im allgemeinen $\mathrm{zu}$ verfahren hat. Dass dies sein wirklicher Zweck ist, und dass er nur aus Mangel an einem Zeichen für eine bekannte aber beliebige Zahl dahingebracht ist diesen bestimmten Wert einzuführen, das erkennt man daraus, dass er in solchen Fällen, wo sich nicht jede Zahl benutzen lässt, dies ausdrücklich in einem Diorismus zu der betreffenden 
Aufgabe auseinandersetzt ${ }^{1}$. Die Einführung eines bestimmten Wertes wendet Diophant auch oft versuchsweise bei der betreffenden Unbekannten an. Wenn es sich dann zeigt, dass dieser nicht passt, so kann er, wenn er dem Gange der vorgenommenen Rechnungen folgt, erkennen, welche Änderung des versuchsweise angenommenen Wertes wirklich $\mathrm{zu}$ der gegebenen Form oder dem gegebenen Werte einer anderen Grösse führen würde. Die Regel des falschen Ansatzes (regula falsi), die wir bereits bei den Ägyptern getroffen haben, ist eine sehr einfache Anwendung dieser Methode, aber Diophant wendet sie in weit mehr verwickelten Fällen an.

Bei den Rechnungen vor- und rückwärts, die solche Versuche erfordern, bedarf es grosser Fertigkeit im Umgehen mit Zahlen und im Überblicken der mit diesen vorgenommenen Operationen. Eine solche Fertigkeit legt Diophant auch vor allem an den Tag im Gegensatz zu dem, was in den überlieferten Arbeiten der alten griechischen Mathematiker vorliegt. Diese unmittelbare Fertigkeit reicht indessen nicht überall aus. $\mathrm{Da}$ die geometrische Darstellung fortgefallen war, so bedurfte es anderer Mittel um eine unbekannte Grösse und einfache Funktionen von ihr festzuhalten. Das erreicht Diophant durch eine algebraische Zeichensprache. Ist diese auch nur noch eine Abkürzung der Worte der Schriftsprache und keineswegs $\mathrm{zu}$ einem wirklichen Organ für die algebraischen Operationen bestimmt, so dient sie doch $\mathrm{zu}$ dem, was man zuallererst von einer Zeichensprache

${ }^{1}$ Der allgemeinere Überblick, den Diophant so, wie sich zeigt, an manchen Stellen gehabt hat, wo er sofort bestimmte Zahlen einführt, wird in den Anmerkungen zu Wertheims neuer deutscher Ausgabe durch Einführung von allgemeinen Bezeichnungen für diese Zahlen deutlich gemacht. 
verlangen muss: einen rascheren und bequemeren Überblick zu gewähren, als man ihn durch eine Darstellung in Worten erhalten kann.

Die Unbekannte wird durch den Buchstaben $\varsigma$ bezeichnet, der gewählt werden musste, weil er allein keine bestimmte Bedeutung als Zahl hatte. Ihre Potenzen bis zur sechsten hinauf werden durch Abkürzungen der griechischen Wörter für Quadrat u. s. w. bezeichnet, und ähnliche Bezeichnungen werden für Brüche gebraucht mit dem Zähler 1 und diesen Grössen als Nennern. So ergeben sich Bezeichnungen für

$x^{-6}, x^{-5}, x^{-4}, x^{-3}, x^{-2}, x^{1}, x, x^{2}, x^{3}, x^{4}, x^{5}, x^{6}$

ausser einer besonderen Bezeichnung für Einer (also für $x^{0}$ ). Mehrgliedrige Grössen, die aus den hier genannten und mit Zahlenkoefficienten multiplicierten Grössen zusammengesetzt sind, lassen sich dadurch auf eine leicht übersichtliche Weise aufschreiben, indem man die zu addierenden Glieder unmittelbar neben einander setzt und ein umgekehrtes $\psi$ wie unser Minuszeichen gebraucht, und einander gleich setzen. Es werden bestimmte Regeln für die Multiplikation der oben genannten Potenzen gegeben, und dadurch lassen sich wieder mehrgliedrige Grössen multiplicieren. Gleichfalls versteht Diophant aus Gleichungen neue Gleichungen dadurch zu bilden, dass er Glieder, Faktoren und Divisoren von der einen der beiden gleichen Grössen zu der anderen hinüberbringt.

Ein wesentlicher Übelstand bei dieser Zeichensprache liegt darin, dass es nur ein Zeichen für eine einzige Unbekannte giebt und daneben wieder besondere Zeichen für deren verschiedene Potenzen. Eine Erweiterung auf zwei Unbekannte würde eben, weil die letzten Zeichen besondere sind, 12 neue Zeichen erfordern, und an solche ist gar nicht gedacht worden. Indessen giebt, wie es so 
oft zu geschehen pflegt, die Mangelhaftigkeit des Werkzeuges Gelegenheit persönliche Fertigkeit zu zeigen. Solche Fertigkeit legt Diophant an den Tag, nicht nur durch Benutzung der oben angeführten Versuchswerte für die Unbekannten, die er nicht benennen kann, sondern auch auf andere Weise. Wenn in einer Aufgabe mehrere unbekannte Grössen vorkommen, die nach verschiedenen Angaben bestimmt werden sollen, so wählt er unter diesen Unbekannten diejenige, worauf er seine Bezeichnung die wir hier $x$ nennen wollen - anwenden will, so, dass er von Anfang an die übrigen durch diese ausgedrückt erhalten kann. Zugleich muss angeführt werden, dass die Bezeichnungen nicht die ganze Aufgabe hindurch festgehalten werden. So kann eine Unbekannte da sein, die von Anfang an $x$ genannt wird, in den folgenden Zwischenrechnungen kann eine zweite und dritte ebenso genannt werden, und wenn man nach der Bestimmung von diesen wieder zu der Hauptaufgabe zurückkehrt, so wird die erste Unbekannte wieder ebenso genannt. Aus diesen Bemerkungen ergiebt sich, dass Diophant im wesentlichen dasjenige, was wir Eliminationen nennen, im Kopf ausführen und in Worten darstellen musste; aber gerade dadurch erhielt er Übung darin seine Unbekannten so $\mathrm{zu}$ wählen, dass die Elimination so einfach wie möglich wurde.

Man könnte glauben, dass das Fehlen von mehr Bezeichnungen für die Unbekannten besondere Unzuträglichkeiten für die unbestimmten Aufgaben mit sich führen würde. Das ist jedoch nicht der Fall, denn diese laufen gewöhnlich darauf hinaus, dass eine zusammengesetzte Grösse ein Quadrat sein soll oder dergleichen. Für die Quadratwurzel u. s. w. hieraus bedarf es dann keiner besonderen Bezeichnung. 
Diese unbestimmten Aufgaben, für die rationale Lösungen gesucht werden, verdienen die grösste Aufmerksamkeit in Diophants arithmetischer Arbeit. In der Regel betrachtet er es jedoch nur als seine Sache eine einzige Lösung der Aufgabe $z u$ finden, und nicht eine solche allgemeine Lösung, in der alle möglichen einzelnen Lösungen einbegriffen sind. Auf diese Beschränkung darf man jedoch kein zu einseitiges Gewicht legen, wenn man recht verstehen will, was Diophant mitzuteilen hat. Sie beruht nämlich in der Regel darauf, dass er auch hier den Hülfsgrössen, durch welche die Aufgabe gelöst wird, sofort bestimmte Werte beilegt; er hat dann aber hier ebenso gut wie in den früheren Fällen sehen können, dass man auch andere Werte der Hülfsgrössen hätte benutzen können. Dies ausdrücklich zu erkennen $\mathrm{zu}$ geben findet er Gelegenheit, wenn eine auf eine gewisse Weise gebildete Grösse zu gleicher Zeit ein Quadrat sein und noch eine andere Bedingung erfüllen soll. Da genügt es nicht, der Hülfsgrösse, die sie zu einem Quadrat machen soll, einen bestimmten Wert zu geben; diese wird dagegen selbst eine unbekannte Grösse $x$, und durch diese muss Di ophant dann im allgemeinen die ursprünglich gesuchten Grössen ausdrücken, um hinterher $x$ durch die zweite gegebene Bedingung $\mathrm{zu}$ bestimmen.

Von den unbestimmten Gleichungen, zu deren Lösung Diophant Gelegenheit erhält, gehören eine grosse Menge unter die Formen

$$
\text { und } \quad \begin{aligned}
& y^{2}=a^{2} x^{2}+b x+c \\
& y^{2}=a x^{2}+b x+c^{2} .
\end{aligned}
$$

Die erste wird, indem wir, um die Darstellung abzukürzen, die jetzige Zeichensprache zu Hülfe nehmen, dadurch gelöst, dass man $y=a x+z$ setzt, die letzte dadurch, dass man $y=z x+c$ setzt; danach lässt sich $x$ leicht rational 
29. Die spätere griechische Arithmetik; Diophant.

durch $z$ ausdrücken, und $z$ kann seinerseits alle rationalen Werte annehmen (nur dürfen diese keine Grösse negativ machen). Man sieht, dass die angewandten Substitutionen dieselben sind, die jetzt benutzt werden um irrationale Differentiale rational $\mathrm{zu} \cdot$ machen.

Auf die letzte der angeführten Gleichungsformen lassen sich die zusammengehörigen Gleichungen, oder wie Diophant sagt «die doppelte Gleichung»

$$
\left.\begin{array}{l}
y^{2}=a x+b^{2} \\
z^{2}=c x+b^{2}
\end{array}\right\}
$$

zurückführen. Ja das letzte Glied braucht nicht einmal in beiden Gleichungen dasselbe zu sein, wenn es nur eine Quadratzahl ist; denn dann kann man es dahin bringen, dass es denselben Wert erhält, indem man die eine Gleichung mit einem Quadrat multipliciert. Der Einfachheit wegen haben wir angenommen, dass dies bereits geschehen sei. Durch Subtraktion der Gleichungen erhält man, wenn man zugleich $x$ durch $z$ ausdrückt,

$$
y^{2}-z^{2}=\frac{a-c}{c}\left(z^{2}-b^{2}\right)=\frac{a-c}{c}(z-b)(z+b) .
$$

Setzt man hierin $z=t+b$, so erhält man

$$
y^{2}=\frac{a}{c} t^{2}+\frac{2 a b}{c} t+b^{2}
$$

und diese Gleichung ist von der oben stehenden Form (2).

Durch andere ähnliche Kunstgriffe hat Diophant auch gewusst wenigstens einzelne rationale Lösungen von anderen zusammengehörigen Gleichungen $\mathrm{zu}$ finden, die in den Formen

$$
\left.\begin{array}{l}
y^{2}=a x^{2}+b x+c \\
z^{2}=d x^{2}+e x+f
\end{array}\right\}
$$


einbegriffen sind, jedoch nur von solchen, bei denen gleichzeitig $c$ und $f$, oder $a$ und $d$ Quadratzahlen sind.

Da wir wie erwähnt nur durch die Behandlung einer Reihe einzelner Aufgaben erfahren, wie Diophant im ganzen verfährt, so wird es zweckmässig sein ein Beispiel von einer solchen Aufgabe und ihrer Behandlung zu geben. In der 6ten Aufgabe des 6ten Buches wird ein solches rechtwinkeliges Dreieck gesucht, dass die Summe aus dem Inhalt und einer Seite, ausgedrückt in (rationalen) Zahlen, eine gegebene Zahl ist. Um es deutlich zu machen, wo Diophant seine Zeichensprache gebraucht und wo nicht, wollen wir bei der Wiedergabe $x$ und $x^{2}$ statt Diophants eigenen Zeichen schreiben, während die übrigen Buchstaben Zahlen ausdrücken, von denen Diophant in gewöhnlichen Worten spricht. Die Aufgabe läuft dann darauf hinaus rationale Werte von $A, B$ und $C \mathrm{zu}$ finden, die den Gleichungen

und

$$
\begin{aligned}
A^{2}+B^{2} & =C^{2} \\
\frac{1}{2} A B+A & =a,
\end{aligned}
$$

wo $a$ eine gegebene Zahl bedeutet, genügen. Dieser Zahl $a$ giebt Diophant jedoch sofort den Wert 7. Versuchsweise wird darauf $A=3 x, B=4 x, C=5 x$ gesetzt, wodurch der ersten gegebenen Gleichung genügt wird. Die zweite liefert dann

$$
6 x^{2}+3 x=7 .
$$

Die Bedingung dafür, dass diese Gleichung rationale Wurzeln haben soll, ist die, dass $\frac{9}{4}+6.7$ quadratisch ist. Das ist zwar nicht der Fall, aber die Rechnung mit den bestimmten Zahlen hat Diophant Gelegenheit gegeben zu sehen, wie diejenige Grösse, die ein Quadrat sein soll, aus Grössen zusammengesetzt ist, die den Seiten des Drei- 
29. Die spätere griechische Arithmetik; Diophant.

ecks proportional sind. Er erreicht also dasselbe, was wir erreichen würden, indem wir

$$
A=\alpha x, B=\beta x, C=\gamma x
$$

setzen, nämlich dass die Bedingung,

$$
\frac{\alpha^{2}}{4}+\frac{\alpha \beta}{2} \cdot 7=\text { einem Quadrat }
$$

die Bedingung für rationale Lösungen ist.

Da es nur auf das Verhältnis der Seiten ankommt, so kann man hier $\alpha=1$ setzen. Diophant wählt darauf vorläufig $\beta$ zur Unbekannten $x$, und als Ergebnis aus den versuchweise gewählten Werten erhält er dann, dass

$$
1+14 x=D^{2} \text {. }
$$

Nach der ersten gegebenen Gleichung soll zugleich

$$
1+x^{2}=E^{2}
$$

sein. Diese zusammengehörigen Gleichungen gehören unter die oben angeführte allgemeine Form (4). Nachdem Diophant hieraus die Gleichung

$$
x(x-14)=E^{2}-D^{2}
$$

abgeleitet hat, schliesst er, offenbar dadurch, dass er die rechte Seite in Faktoren zerlegt und

$$
E+D=x, E-D=x-14
$$

setzt, dass $D$ die halbe Differenz der Faktoren oder gleich 7 ist, und danach wird $x=\frac{24}{7}$.

Dieser letzte Wert stellt das Verhältnis zwischen den Katheten dar. Indem darauf Diophant $x$ eine ähnliche Bedeutung annehmen lässt wie ursprünglich, setzt er in die zweite gegebene Gleichung

$$
A=7 x, B=24 x
$$

ein; daraus ergiebt sich 


$$
7.12 x^{2}+7 x=7,
$$

woraus $x=\frac{1}{4}, A=\frac{7}{4}, B=6$ und $C=\frac{25}{4}$.

Um eine deutlichere Vorstellung von Diophants zahlreichen Aufgaben zu geben, wollen wir noch aufs Geratewohl ein paar Beispiele auswählen und kurze Angaben aus seinen Lösungen hinzufügen.

II, 20. Drei Quadratzahlen von der Beschaffenheit zu finden, dass die Differenz zwischen der grössten und mittleren in einem gegebenen Verhältnis zu der Differenz zwischen der mittleren und kleinsten steht. - Nennt man die kleinste $x^{2}$, die mittlere $(x+a)^{2}$, so wird die grösste

$$
(x+a)^{2}+m\left[(x+a)^{2}-x^{2}\right] .
$$

Dass diese ein Quadrat sein soll, wird durch eine Gleichung der oben angeführten Form (1) ausgedrückt. Diophant begnügt sich mit $m=3$ und $a=1$.

III, 2. Drei Zahlen von solcher Beschaffenheit zu finden, dass das Quadrat ihrer Summe neue Quadrate giebt, wenn man jede der Zahlen dazu addiert. - Diophant lässt die Summe $x$ sein. Die Bedingungen sind dann erfüllt, wenn die Zahlen $\left(a^{2}-1\right) x^{2},\left(b^{2}-1\right) x^{2}$ und $\left(c^{2}-1\right) x^{2}$ sind, und wenn

$$
\left(a^{2}-1\right) x^{2}+\left(b^{2}-1\right) x^{2}+\left(c^{2}-1\right) x^{2}=x,
$$

woraus sich ein rationaler Ausdruck für $x$ ergiebt. Diophant nimmt für $a, b$ und $c$ nur die Werte 2,3 und 4 .

IV, 27. Zwei Zahlen von der Beschaffenheit $\mathrm{zu}$ finden, dass ihr Produkt, vermehrt um jede der Zahlen, ein Kubus wird. - Diophant setzt die erste Zahl gleich $a^{3} \varkappa$ (und wählt für $a$ den Wert 2), die zweite gleich $x^{2}-1$, wodurch die eine Bedingung unmittelbar erfüllt wird. Nun soll aber noch

$$
y^{3}=a^{3} x^{3}+x^{2}-a^{3} x-1
$$


29. Die spätere griechische Arithmetik; Diophant.

sein. In Übereinstimmung mit der Art und Weise, wie die unbestimmten quadratischen Gleichungen (1) und (2) gelöst werden, wird diese kubische dadurch gelöst, dass $y=a x-1$ gesetzt wird; dadurch ergiebt sich eine Gleichung ersten Grades zur Bestimmung von $x$.

Wir wollen noch erwähnen, dass einzelne Aufgaben Diophant Gelegenheit geben, seine Bekanntschaft mit gewissen zahlentheoretischen Sätzen zu zeigen, so mit dem Satze, dass eine Zahl von der Form $\left(a^{2}+b^{2}\right)\left(c^{2}+d^{2}\right)$ sich auf zwei Arten in die Summe von zwei Quadraten zerlegen lässt, nämlich

$$
(a c \mp b d)^{2}+(a d \pm b c)^{2},
$$

und mit dem anderen, dass eine Zahl von der Form $4 n+3$ sich auf keine Weise in eine Summe von zwei Quadraten zerlegen lässt.

Die angeführten Beispiele werden gezeigt haben, dass Diophant nur rationale (und selbstverständlich positive) Lösungen sucht, aber nicht eben Lösungen in ganzen Zahlen. Es beruht deshalb auf einem Irrtum, wenn man unbestimmte Gleichungen ersten Grades, die sich durch ganze Zahlen sollen lösen lassen, «diophantische Gleichungen» genannt hat. Unbestimmte Gleichungen ersten Grades finden sich allerdings bei Diophant; aber er trägt nur Sorge dafür anzugeben, wie die eine Unbekannte durch die andere ausgedrückt wird, da die Rationalität von dieser diejenige der anderen mit sich bringt. Diese Aufgaben wurden für den Herausgeber Diophants im 17 ten Jahrhundert, Bachet de Méziriac, die Veranlassung, selbständig die Frage nach ganzen Auflösungen zu stellen und diese Aufgabe zu lösen. Sie war jedoch schon früher von indischen Schriftstellern gelöst, was Bachet nicht wusste. 
Nun erhebt sich die Frage, wieviel von Diophants Arbeit von ihm selbst herrührt, und wie alt das übrige ist. Um dies $\mathrm{zu}$ beantworten hat man nicht viele Anhaltspunkte. Wir haben hervorgehoben, dass man bereits zu der Zeit, wo die übrige griechische Mathematik entstand, einzelne Aufgaben von derselben Natur behandelt hat wie diejenigen, die Diophant beschäftigen. Dass uns in den überlieferten Schriften nicht mehr entgegentreten, lässt sich sehr wohl durch den Umstand erklären, dass sie nach der Natur dieser Schriften nicht in sie hinein gehörten. Indessen glaube ich nicht, dass viele von Diophants Aufgaben aus dieser Zeit herstammen. Demn wir haben nicht den Eindruck erhalten kömnen, dass die älteren griechischen Mathematiker die Rechenfertigkeit besassen, die uns bei Diophant entgegentritt. Auf der anderen Seite rührt eine so grosse Sammlung verschieden gearteter Aufgaben, wie diejenige des Diophant, sicherlich nicht von einem einzelnen Manne her. Deshalb liegt die Annahme am nächsten, dass die Bildung dieser Aufgaben auf einem sehr frühen Standpunkt begonnen hat, wahrscheinlich gleich nach der Entdeckung der irrationalen Grössen, und sich dann über die Zeit hinaus, wo sonst die Entwickelung der griechischen Mathematik in Stillstand geraten war, fortgesetzt hat, vielleicht bis zu Diophant hinauf, der in dieser Beziehung recht wohl grosse persönliche Verdienste gehabt haben kann. Dass eine solche Fortsetzung der Entwickelung bei einem einzelnen Zweige der Mathematik hat stattfinden können, kann darauf beruhen, dass die hierfür wichtige Rechenfertigkeit sich nach und nach entwickelt hat, teils wegen der Bedürfnisse der Astronomie und teils durch Berührung mit einem aıderen Volke, den Indern. Mit diesen trat namentlich Alexandria durch seinen Handel in Verbindung. Wie wir sehen werden besassen nämlich die 
29. Die spätere griechische Arithmetik; Diophant.

Inder auch bevor sie das Positionssystem, d. h. die jetzt gebräuchliche Art Zahlen zu schreiben, erfunden hatten, eine grosse Fertigkeit darin Zahlen zu benennen, darzustellen und mit ihnen zu rechnen. Diese Fertigkeit konnte besonders durch die Handelsverbindungen den Griechen übermittelt werden, die damals noch einen Teil der mathematischen Bedingungen für ihre Benutzung besassen. Als Gegengabe haben die Inder dann bei derselben Berührung einen Teil der mathematischen Resultate der Griechen empfangen. Diese haben die Inder, wie wir gleichfalls sehen werden, $\mathrm{zu}$ benutzen verstanden, namentlich da, wo sie sich in Zahlenoperationen umsetzen liessen, aber ohne dass sie jemals verstanden hätten in die strengen theoretischen Begründungen der Griechen einzudringen.

Was im besonderen Diophants Zeichensprache betrifft, die teilweise von derjenigen abweicht, die wir bei viel jüngeren indischen Schriftstellern, deren Arbeiten uns erhalten sind, antreffen, so brauchen wir darin keine Entlehnung von Fremden zu sehen. Die Abkürzungen, die er benutzt, müssen sich von selbst darbieten, sobald man ohne Benutzung der älteren geometrischen Darstellung die nach und nach gebildeten Zusammensetzungen von bekannten und unbekannten Zahlen anderen mitteilen, oder auch nur selbst festhalten will. Hat man sie einmal niedergeschrieben, so wird ihr grosser Nutzen als Mittel um Überblick zu gewinnen sich von selbst gezeigt haben. Diophants Zeichensprache braucht also gar nicht durch eine successive Entwickelung entstanden zu sein; sie kann sehr wohl von ihm selbst oder einem einzelnen Vorgänger herrühren.

Endlich wollen wir bereits hier einen Blick auf die Bedeutung werfen, die Diophants Arbeiten später erlangt haben. Die numerische Beschaffenheit von Diophants bestimmten Gleichungen ersten und zweiten Grades musste 
sie denjenigen viel leichter zugänglich machen, die nicht von vornherein in die griechische Mathematik eingeweiht waren, und zwar leichter zugänglich als die abstrakten geometrischen Formen, in denen namentlich die Gleichungen zweiten Grades bei Euklid vorkommen, und in denen diese und Gleichungen ersten Grades in den überlieferten Schriften anderer geometrischer Schriftsteller angewandt werden. Deshalb war es im wesentlichen Diophant, von dem aus die griechische Algebra zu den Arabern verpflanzt wurde, von denen aus sie wieder beim Wiedererwachen der Wissenschaften in Europa hierher zurückkehrte. Wieviel Einfluss Diophants Behandlung der unbestimmten Gleichungen auf diejenige der Inder, die wir bald erwähnen werden, ausgeübt hat, weiss man nicht; arabische Schriftsteller haben in der von ihm angegebenen Richtung weiter gearbeitet, und in Europa nahmen zahlentheoretische Studien einen neuen Aufschwung, als man solche nicht nur durch die Araber kennen lernte, sondern auch mit Diophants eigener Arbeit bekannt wurde. In dieser Beziehung genügt es daran $\mathrm{zu}$ erinnern, dass Fermat den Diophant sehr gründlich studiert hat. 


\section{Die indische Mathematik.}

\section{Kurzer Überblick.}

Wir wenden uns nun der indischen Mathematik zu, die in ganz anderen Richtungen als die griechische einen ausserordentlichen Einfluss auf die Entwickelung der Mathematik ausgeübt hat. Dieser Einfluss hat sich allerdings wohl am meisten durch unmittelbare Mitteilung der indischen Rechenkunst geltend gemacht, und nur in geringem Grade durch die Schriftsteller, die wir nun erwähnen wollen. Immerhin sind es jedoch diese, denen wir die unmittelbarste Kenntnis dessen verdanken, was die indischen Mathematiker überhaupt wussten und vermochten. Die Schriftsteller haben im Sanskrit geschrieben, einer Sprache, die damals schon eine tote Sprache war, die aber von den Brahmanen bei religiösen und wissenschaftlichen Arbeiten angewandt wurde, ebenso wie später von den Europäern das Lateinische.

Die ältesten von diesen Schriftstellern geben geometrische Regeln für die Anlage von Tempeln, die von ähnlicher Art sind wie die von den Harpedonapten im alten Ägypten benutzten (S 12). In diesen Regeln treffen wir mehrere Spuren der Beeinflussung durch die griechische Geometrie, namentlich einzelne solche Umformungen, die wir von der geometrischen Algebra her 
kennen. Im übrigen müssen wir bei den indischen Astronomen die Kenntnis von denjenigen Seiten der indischen Mathematik suchen, die durchgreifende Bedeutung erlangt haben. Diese Kenntnis wird entwickelt um der Astronomie zur Hülfe zu kommen, oder sie tritt gar nur gelegentlich innerhalb der Darstellung der Astronomie hervor.

Das letzte gilt namentlich von einer Schrift Sûrya Siddhânta aus dem 4ten oder 5ten Jahrhundert n. Chr, deren Verfasser nicht bekannt ist. Âryabhatta, geboren $476 \mathrm{n}$. Chr., hat in ein astronomisches Werk einen mathematischen Abschnitt mit aufgenommen. Von grösserer Bedeutung in mathematischer Beziehung sind das 12te und 18te Kapitel in einer grossen astronomischen Schrift von Brahmagupta, geb. 598. Eine vollständigere Kenntnis der Einzelheiten der indischen Mathematik erhalten wir durch die weit jüngeren beiden mathematischen Arbeiten des Bhâskara Âcârya (des Gelehrten), geb. 1114, welche die Titel Lîlâvatî (die Schöne) und Vîjaganîta (Wurzelberechnung) tragen. Die erstere behandelt etwa das, was wir Rechenkunst und Arithmetik nennen, der Inhalt der zweiten entspricht so ziemlich unsere Algebra. Der Name des ersten dieser Werke ist wohl so zu verstehen, dass die Rechenkunst selbst, die in den Aufgaben in sehr poetischen Ausdrücken angeredet wird, die Schöne genannt wird. Diese Anrede stimmt gut zu dem poetischen Schwung, den die Aufgaben oft haben. Úbrigens giebt es eine Legende, nach der es die eigene Tochter ist, die Bhâskara durch die anziehenden Rechenaufgaben über eine bittere Enttäuschung trösten will.

Wenn auch die Inder eine uralte Astronomie gehabt haben mögen, die sich vermutlich an die chaldäische angeschlossen hat, so zeigt doch bereits Sûrya Siddhânta 
so starke Beeinflussungen entweder durch Ptolemäus oder durch ältere griechische Astronomen, dass sich die möglicherweise ursprünglich indischen Bestandteile nicht mehr ausscheiden lassen. Griechischer Einfluss auf die indische Kultur - und umgekehrt - reicht gewiss zurück bis auf Alexanders Zug nach Indien, und hat sich später fortgesetzt teils durch einzelne Kolonien, teils durch die Handelsverbindungen, für die Alexandria ein Mittelpunkt war. Die den Griechen bekannten mathematischen Sätze und Operationen, die wir auch bei den Indern treffen, verdanken diese gewiss jenen, aber die Inder entwickeln darin Seiten, die eine sehr viel weiter gehende numerische Behandlung erfordern, als die Griechen bei ihrer streng theoretischen Behandlung jemals erreicht haben. Für eine solche Behandlung scheinen die Inder keinen Sinn gehabt zu haben, aber dafür waren sie auch vollständig frei von den Bedenken, welche die griechischen Mathematiker dahin brachten, die wirkliche Berechnung in Zahlen deshalb für geringer zu halten, weil sie oft nur zu einer Annäherung führt. Numerische Berechnung und die dadurch erhaltene praktische Prüfung war im Gegenteil für die Inder das wirkliche Mittel sich Sätze und Methoden anzueignen, von deren eigentlicher Begründung sie die Bedeutung kaum vollständig verstanden. Jedenfalls geben sie solche Begründungen nicht in Worten wieder, sondern sie begnügen sich damit zu zeichnen und durch das Wort «Siehe!» auf die Figur hinzuweisen, die der wirklichen Beweisführung der Griechen zu Grunde lag.

Von weit grösserer Bedeutung als die Entwickelung, die verschiedene Zweige der Mathematik durch die von den Indern angewandte numerische Berechnung erfuhren, ist jedoch die Schreibung der Zahlen und die Rechenkunst, die wir ihnen verdanken. Es ist dieselbe Schreibung der Zahlen mit Stellenwert für die einzelnen Ziffern 
( Positionssystem»), die wir heutigen Tages benutzen, und im wesentlichen dieselbe daran angeschlossene mechanische Ausführung der Berechnungen. Leider ist nur wenig darüber bekannt, wie dieses System sich gebildet hat, da die zur Vollendung des Systemes dienende zehnte Ziffer 0 sich bereits in Sûrya Siddhânta findet. Viel älter scheint das Positionssystem jedoch nicht zu sein, wenn auch die neun ersten, eigenen Wert besitzenden Ziffern auf. weit älteren Inschriften vorkommen. Etwas weitere Aufklärung über die frühere Behandlung der Zahlen bei den Indern lässt sich jedoch insofern in der überlieferten Litteratur finden, als ältere Methoden Zahlen zu schreiben oft beibehalten sind, entweder aus Pietät, oder wegen der besonderen Vorteile, die durch diese Methoden des Schreibens sich darboten; auf diese Weise aber kann man nur ein sehr unvollständiges Bild von der Vorgeschichte des Positionssystemes bei den Indern erhalten. Diesem Mangel wollen wir dadurch etwas ab. zuhelfen versuchen, dass wir der Darstellung des Positionssystemes bei den Indern einen ganz kurzen Überblick über dessen allgemeine Vorgeschichte als Einleitung voranschicken. Wenn wir in aller Allgemeinheit die Hülfsmittel erwähnen, deren man sich bei der Berechnung von Zahlen vor der Erfindung des Positionssystemes, oder doch bevor dieses an den betreffenden Stellen bekannt wurde, bediente, und wenn wir unter diesen die ziemlich dürftigen Mittel berühren, mit denen sich selbst die Griechen begnügen mussten, so werden wir jedenfalls eine Vorstellung davon geben können, wie grosse Schwierigkeiten es verursacht hat zu diesem System zu gelangen. Von wie grosser Bedeutung dieses System ist, keineswegs allein als Teil der Mathematik, sondern für die Menschheit in den alltäglichsten Dingen, bedarf keines näheren Nachweises. 


\section{Zahlbenennung, Zahlbezeichnung und Zahlen- rechnen vor und bei den Indern.}

Wenn eine Mutter einen Apfel für jedes von ihren 7 Kindern nehmen will, so braucht sie die Zahl 7 nicht $\mathrm{zu}$ kennen. Ebenso wenig braucht sie zu wissen, dass $2.7=14$, un jedem Kinde zwei zu geben. Sie nimmt ganz einfach je einen oder zwei für Hans, Louise u. s. w. Sie kommt dem Zahlenbegriffe näher, wenn sie beachtet hat, dass sie im ersten Falle einen Apfel für jeden Finger der einen Hand und für den ersten und zweiten Finger der andern Hand nehmen muss. Die Finger, die nichts mit den Dingen zu thun haben, von denen hier ebenso viele sein sollen, werden für sie dann nur Bezeichnungen für die Anzahl von diesen.

Dass die Völker sich auf diesem Wege zum Zahlenbegriffe erhoben haben, geht daraus hervor, dass sie sich fast alle um grössere Zahlen zu benennen des Zehner-, Fünfer- (zunächst wohl nur als Durchgangsglied zum Zehnersystem) oder Zwanzigersystemes bedienen. Diese sind, wenn es auch lange Zeit gedauert haben mag, von selbst $\mathrm{zu}$ wirklichen Systemen geworden. Nachdem man alle Finger durchgezählt hatte, oder weiter gegangen war und, wie ein Volk am Orinoco 20 ausdrückt, einen ganzen Menschen (d. h. Finger und Zehen) gezählt hatte, hat man von vorne beginnen und dann darauf achten müssen, wie viele Zehner oder Zwanziger, und wie viele Einer man über diese hinaus gezählt hatte. Dadurch sind die Zahlbildungen entstanden, die wir durch $a+b x$ bezeichnen können, wo $x$ die höhere Einheit, Zehner oder Zwanziger, bezeichnet. Wenn die Entwickelung und Benutzung der Zahlen so weit gekommen war, dass die Anzahl der 
Zehner oder Zwanziger selbst 10 oder 20 überschritt, so hat man höhere potentielle Einheiten, $10^{2}, 10^{3} \ldots$, oder wie die alten Azteken in Mexiko $20^{2}, 20^{3} \ldots$, bilden und Zahlen von den Formen

$$
a+b x+c x^{2}+\ldots
$$

darstellen müssen.

Wie weit man gegangen ist, hat sich dann nach dem Bedarf richten müssen. Die unbegrenzte Möglichkeit für die Bildung höherer Einheiten ist dagegen in der Regel erst auf einem mehr wissenschaftlichen Standpunkte geltend gemacht worden, wie von Archimedes in der Sandrechnung.

So sind die Zehner- und Zwanzigersysteme gebildet, das letztere zunächst wohl von Völkern, die immer, oder wie die Grönländer in ihrer Wohnung, nackt oder mit nackten Füssen gehen. Die deutlichen Spuren des Zwanzigersystemes, die sich in mehreren europäischen Sprachen und nicht zum wenigsten im Dänischen finden, sind dagegen späteren Ursprunges und wahrscheinlich dadurch entstanden, dass die grössere Einheit, das Stieg (20 Stück), in gewissen Fällen für Handel und Wandel bequem gewesen ist. Neben den hier genannten höheren Einheiten hat man bei der Einteilung von Münzen, Maassen und Gewichten oft andere benutzt, die sich aus mehr theoretisch Gründen als bequemer erwiesen haben, wie $12=$ $2^{2}$.3. Ein Beispiel für eine noch weiter gehende Rücksichtnahme auf derartige Gründe ist das aus Babylonien stammende Sexagesimalsystem, das wir bereits erwähnt haben.

Ausser Addition, Multiplikation und den ersten Potenzerhebungen, die, wenn auch unbewusst, diesen Zahlenbildungen $\mathrm{zu}$ Grunde liegen, hat man bei den Zahlenbenennungen auch Subtraktion benutzt, so wenn 
19 im Lateinischen un-de-viginti heisst, im Sanskrit eleonavimçati (d. h. 20-1) oder nur ûnavimçati (d. h. mangelhaft 20).

Heute gebrauchen wir, sowohl um mit den so gebildeten Zahlen zu rechnen als um sie aufzuschreiben, ein und dasselbe Hülfsmittel, aber so ist es nicht immer gewesen. Zum augenblicklichen Festhalten der Zahlen, die beim Rechnen erforderlich waren, hat man zuerst dasselbe Hülfsmittel wie beim Zählen benutzen können, nämlich die Finger. Die verschiedenen Einheiten wurden bei einem afrikanischen Volke dadurch festgehalten, dass ein Mensch einen Finger in die Höhe halten musste für jede einfache Einheit, ein zweiter für jeden Zehner, ein dritter für jeden Hunderter; ein einzelner Mensch aber kann für denselben Zweck auf verschiedene Weise die Glieder der Finger verwenden (Fingerrechnung bei Griechen und Römern). Andere mechanische Hülfsmittel, die man an sehr verschiedenen Stellen der Erde angewandt hat und noch anwendet, die von den alten Griechen und Römern und im Mittelalter in Europa benutzt wurden, und die auch civilisierte Völkerschaften heute noch für besondere Zwecke (Berechnungen beim Kartenspiel) oder als Lehrmittel in Kinderschulen benutzen, sind Rechenbretter, einfache Rechenmaschinen und Rechenpfennige. Auf den Rechenbrettern findet sich eine Einteilung in Kolumnen für Einheiten derselben Art, deren Anzahl dann durch aufgelegte Steinchen oder andere Marken bezeichnet wird. Auf den Maschinen, die in unserem Jahrhundert von asiatischen Völkern, die sie lange gebraucht haben, nach Europa gekommen sind, sind die Kolumnen mit Saiten oder Stäben vertauscht, auf denen sich Kugeln oder dergleichen verschieben lassen. Jede Kolumne oder jeder Stab kann aus zwei Unterabteilungen bestehen, von denen die eine 4 oder 5 Marken zur Be- 
zeichnung von Einheiten einer gewissen Art enthällt, die andere 1 oder 2 Marken zur Bezeichnung fünfmal so grosser Einheiten. Von Rechenpfennigen giebt es verschiedene Formen um Einheiten verschiedener Art zu bezeichnen. Dass diese Hülfsmittel sich benutzen lassen um einfache Rechnungen, Addition, Subtraktion und Multiplikation mit kleineren Zahlen auszuführen, erkennt man leicht, und deshalb wollen wir uns mit einer Untersuchung darïber, wie man das an den verschiedenen Orten gemacht hat, nicht aufhalten.

Man kommt unserem Zahlenrechnnen näher, wenn man die eingeteilten Kolumnen allerdings benutzt, aber statt Marken auf die Kolumnen zu legen Zahlzeichen für $1-9$ in sie hineinschreibt. Hierzu ist nicht nur die Kenntniss der Schreibkunst erforderlich, sondern auch, da man seine Marken nun nicht mehr mechanisch zusammenzählt, zugleich das Einüben von Tabellen (Eins und Eins, Einmaleins) oder die Benutzung geschriebener Tabellen. Man hat das Positionssystem, wenn man statt die im voraus gezeichneten Kolumnen zu benutzen, die geschriebenen Ziffern selbst diese Kolumnen bilden lässt. Dazu bedarf man eines Zeichens, das einen Platz ausfüllt ohne selbst irgend welchen Wert zu besitzen, nämlich 0. Dass die Erfindung der Null nicht ganz von selbst kam, sieht man daraus, dass das Positionssystem so lange auf sich warten liess. Im übrigen hat sich ergeben, dass selbst, nachdem es gefunden war, eine gewisse Entwickelung dazu gehörte um es benutzen zu können. Nicht nur muss man, um ein System von Kolumnen gebrauchen zu können, worin die Zahlen hineingeschrieben werden, schreiben können und einige Tabellen (Eins und Eins, Einmaleins) gelernt haben, sondern man muss auch einigermassen zierlich und gleichmässig schreiben, damit die Ziffern auf ihren rechten Platz kommen, und man muss mehr im 
Gedächtnis behalten, als wenn man überschiessende Einheiten höherer Ordnung und so viele Ziffern, wie man will, in die im voraus gezeichneten Kolumnen hineinschreiben kann.

Mit Ausnahme der beiden letzten haben die hier genannten Mittel keine Ansprüche an die Kenntnis der Schreibkunst gemacht. Dasselbe lässt sich sogar von den ersten Entwickelungsstufen der Zahlenschreibung selbst sagen, die nur in festen Marken statt der beweglichen Steine, Kugeln oder Rechenpfennige bestanden haben. Man hat, ganz so wie es jetzt geschieht, wenn die Einer einzeln kommen, z. B. beim Stimmenzählen, für jeden Einer eine Marke abgesetzt. Man hat solche Marken in neue für 5 oder 10 zusammenfassen können, für die jedoch später selbständigere Marken entstanden sind, ebenso wie für die höheren Einheiten. Auf diese Art kommt man dann zu einer zusammenhängenden Zahlbezeichnung wie diejenige der Römer war — um uns an ein wohlbekanntes Beispiel $\mathrm{zu}$ halten -. Es ist leicht zu begreifen, wie eine derartige Zahlenschreibung entstanden ist, und es würde leicht sein die Bedeutung einer solchen Zahlenschrift $\mathrm{zu}$ finden, selbst ohne irgend welche Anleitung empfangen zu haben.

Die Griechen, die in älteren Zeiten, wie sich aus alten Inschriften ergiebt, die Zahlen auf ähnliche Weise geschrieben hatten, benutzen in der uns überlieferten Litteratur vollkommen entgegengesetzte Principien für die Zahlenschreibung. Jede ganze Anzahl Einer, Zehner und Hunderter hat nämlich ihren eigenen Buchstaben. Man schreibt

$$
\text { für } \begin{array}{ccccccccccc}
1 & 2 & 3 & \ldots & 9 & 10 & 20 & \ldots & 100 & 200 & \ldots \\
\alpha & \beta & \gamma & \ldots & \vartheta & \iota & \varkappa & \ldots & \varrho & \sigma & \ldots
\end{array}
$$


dadurch wird beipsielsweise

$$
803=\omega \gamma, 83=\pi \gamma, 833 \omega \lambda \gamma .
$$

Diese Bezeichnungsart scheint im ersten Augenblick ihres unsystematischen Charakters wegen ein Rückschritt zu sein. Nichts lässt erkennen, was gleichartig ist. So verraten die Zeichen $\beta, \varkappa, \sigma$ durch nichts, dass man es mit gleichviel Einheiten verschiedener Art zu thun hat. Indessen sieht man, dass die Griechen eine $\mathrm{Zahl}$ viel kürzer darstellen als die Römer, und ihre Zahlbezeichnung darf nicht, selbst wenn sie das einzige wäre, was wir von den Griechen kennten, als Zeichen einer niedrigeren Entwickelungsstufe betrachtet werden. In unseren Tagen haben viele Sprachforscher die ältere Ansicht aufgegeben, dass es ein Zeichen für die hohe Entwickelung einer Sprache sein solle, wenn sie wie die lateinische durch vollständige Regeln alle Worte auf solche Formen und in solche Verbindungen mit einander bringt, dass derjenige, der noch keinen Begriff vom $\mathrm{Zu}$ sammenhange hat, aus den Formen schliessen kann, wohin jedes Wort gehört, und sich so den Zusammenhang konstruieren kann. Etwas ganz ähnliches findet man in den Sprachen der am wenigsten entwickelten Völker. Jetzt dagegen sieht man die Vollkommenheit einer Sprache darin, dass sie mit so wenig Mitteln wie möglich, also mit der geringsten Mühe für den Sprechenden und den Hörenden ein vollkommenes Verständnis herstellt. Hierzu gehört, dass man (wie im Englischen) solche Hülfsmittel für die Beurteilung des Zusammenhanges zwischen den einzelnen Worten auslässt, die in Wirklichkeit überflüssig sind. Soll das Verständnis nicht verloren gehen, so wird dann allerdings eine genauere Kenntnis von der Sprache, z. B. von der Bedeutung der Wortstellung, verlangt, wenn Missverständnisse unmöglich sein sollen; ist 
aber diese Kenntnis vorhanden, so gelangt man viel rascher zum Verständnis, als wenn man sich den $\mathrm{Zu}$ sammenhang durch Betrachtung der Übereinstimmung in Genus, Numerus und Casus, konstruieren soll. Einen ähnlichen Vorteil gewährte das Schreiben und Lesen der Zahlen bei den Griechen gegenüber der Weitläufigkeit der römischen Zahlen. Die Griechen konnten die Zahlen bis 1000 nicht nur ebenso kurz schreiben wie wir, sondern für den, der mit den Zeichen vertraut ist, sind ihre Zahlen auch rascher zu lesen, als wenn man sich durch eine römische Zahl hindurch zählen muss.

Als Schriftzeichen für nicht zu grosse Zahlen dürften die griechischen also sehr gut sein. Indessen waren diese Bezeichnungen, vermutlich weil man, wo es nötig war, nebenher mechanische Mittel für das Rechnen benutzte, allzu ausschliesslich nur für die schriftliche Mitteilung bestimmt. Um eine Zahlenschreibung zu erhalten, die zugleich zweckmässig als Rechenmittel und brauchbar für die Darstellung von unbegrenzten Zahlen war, musste man auf dem betretenen Wege wieder umkehren. Für eine solche Zahlenschreibung war eine Vereinigung von griechischer Kürze mit römischer Durchsichtigkeit erforderlich. Einige Annäherung hieran zeigt eine Bezeichnung, welche die Chinesen benutzen: die verschiedenen höheren Einheiten haben jede ihr besonderes Zeichen, und ihre Anzahl wird durch dieselben Ziffern angegeben, die die einfache Anzahl von Einern bezeichnen. Wenn wir die eigenen Zeichen der Chinesen mit einer Kombination der römischen nnd unserer Zahlzeichen vertauschen, so lässt sich die Auwendung dieses Systemes durch die folgenden Bezeichnungen darstellen:

$$
833=8 \mathrm{C} 3 \times 3,803=8 \mathrm{C} 3,83=8 \times 3 .
$$


Einfacher wird das System, wenn man die Art der höheren Einheit durch hinzugefügte Marken angiebt, wie

$$
833=\ddot{8} 33,803=\ddot{8} 3,83=\dot{8} 3 .
$$

Kürze, Klarheit und Anwendbarkeit sind jedoch am besten im Positionssystem vereinigt.

Nachdem wir hier an den am besten bekannten Beispielen erläutert haben, wie die Bildung von Zahlen im allgemeinen vor sich gegangen ist, und zugleich die Wege gezeigt haben, auf denen man zum Rechnen und zum Schreiben der Zahlen gelangt ist, wollen wir noch einen Blick auf das werfen, was wir in dieser Beziehung von den Indern vor der Erfindung des Positionssystemes besonderes wissen.

Dass die Inder sich früh mit grossen Zahlen beschäftigt haben, ergiebt sich daraus, dass sie schon früh Namen für die decimalen Einheiten bis hinauf $\mathrm{zu} 10^{17}$ gebildet haben. Altes Interesse für grosse Zahlen verrät sich ferner dadurch, dass in Legenden von Buddha erzählt wird, er habe derartige Namen bis hinauf $\mathrm{zu} 10^{54}$ gebildet, ja er habe Bildungen von noch höheren Zahlen beabsichtigt. Hieraus und aus der Neigung der Inder zu numerischen Übertreibung geht hervor, dass sie bereits von Alters her das besassen, was Archimedes den Griechen erst in seiner Sandrechnung brachte. In ihren Benennungen für die decimalen Einheiten fehlt es allerdings an solchen Ruhepunkten, wie wir sie in Tausend, einer Million u. s. w. haben. Das ist ein Mangel an System; aber es ist doch, wie bei der Zahlenschreibung der Griechen, ein Zeichen von Entwickelung, dass man sich durch die vielen verschiedenen Bezeichnungen überhaupt verständlich machen konnte. Die bestimmte Absonderung jeder einzelnen decimalen Einheit weist im übrigen auf die 
Principien hin, aus denen das Positionssystem hervorgehen sollte. Diese Principien finden sogar Anwendnng beim Aussprechen von Zahlen. So wird an einer Stelle die Zahl 1577917828 durch eine Mischung von bildlichen Ausdrücken für Zahlen und eigentlichen Zahlen, indem mit den Einern begonnen wird, dargestellt, durch Vasu (d. i. eine Klasse von 8 Gottheiten), 2, 8, Berge (7) Form (1), Ziffern (nämlich die 9), 7, Berge, Mondtage (15, d. i. ein halber Monat). Die letzte Bezeichnung entspricht einer zweiziffrigen mit 1 beginnenden Zahl, und dasselbe kann auch innerhalb einer auf ähnliche Weise zusammengesetzten Zahl stattfinden. Das Hersagen der Zahl geschieht auf solche Weise rascher als bei uns, wenn wir uns nicht auch etwa damit begnügen wollten, die einzelnen Ziffern der Reihe nach zu nennen. Dagegen ergiebt sich die Misslichkeit, dass eine und dieselbe Ziffer, hier beispielsweise sowohl 7 wie 8, verschiedene Namen erhält. Das hängt indessen mit einem Hülfsmittel zusammen, das angewandt wurde um sowohl Zahlen als auch mathematische Regeln im Gedächtnis zu behalten, nämlich sie in Verse zu bringen, ein Mittel, das sicherlich mit den poetischen Neigungen der Hindu in Verbindung stand, das aber auch praktischen Nutzen gewährte.

Das angeführte Beispiel findet sich allerdings bei Brahmagupta, als das Positionssystem also schon längst bekannt war; aber ihrer ganzen Beschaffenheit nach muss diese Art der Benennung, die für die verschiedenen Zahlen alte, nach Übereinkunft gebildete Namen voraussetzt, alt sein. Da in der angeführten Zahl die Null fehlt, so kann sogar dieses Beispiel selbst alt sein.

Was die eigentliche Schreibung der Zahlen betrifft, so haben wir bereits bemerkt, dass die Benutzung der 9 Ziffern sich auf uralten Inschriften findet. Um hieraus 
grössere Zahlen zusammenzusetzen kann man sich eines Verfahrens bedient haben, wie es sich bis vor kurzem auf Ceylon erhalten hat. Dieses besteht darin, dass man teils wie die Griechen die 9 Ziffern durch besondere Zeichen für $10,20,30 \ldots 100$ und demnächst für 1000 ergänzt, teils wie die Chinesen eine gewisse Anzahl von Hunderten dadurch bezeichnet, dass man die betreffende Ziffer vor das Zeichen für 100 stellt, oder man kann auch dem Princip der Chinesen ganz gefolgt sein.

Ungefähr so wie diese verfährt nämlich noch Âryabhatta. Er benutzt die Konsonanten um die Ziffern auszudrücken, und giebt durch einen hinzugefügten Vokal an, von welcher decimalen Einheit diese Anzahl genommen werden soll. So ist

$$
g a=3, g i=30, g u=30000 \text { u.s. w. }
$$

Dadurch erreicht er eine hörbare Darstellung von Zahlen, die in Verse hinein passen kann.

Die Arten des Verfahrens, welche die Inder für das Rechnen benutzten, bevor das eigentliche Positionssystem durch Einführung der Ziffer 0 vollendet worden war, können sehr wohl denen geglichen haben, die man anwandte, nachdem es zur Benutzung gelangt war; denn die Zahlenschreibung, deren man sich bediente, wird es jedenfalls deutlich gemacht haben, wieviele von jeder decimalen Einheit vorhanden waren. Unmittelbar können solche Arten des Verfahrens bei den uns überlieferten Schriftstellern benutzt worden sein, wo die Bedeutung der 9 Ziffern, die man hatte, durch ihren Platz in einem im voraus eingeteilten Rahmen angegeben wird, denn in einem solchen erhält man durchaus keine notwendige Verwendung für das Zeichen 0 . Das gilt z. B. von folgender Form für die Multiplikation von 12.735, die auch auf grössere Zahlen angewandt wurde: 


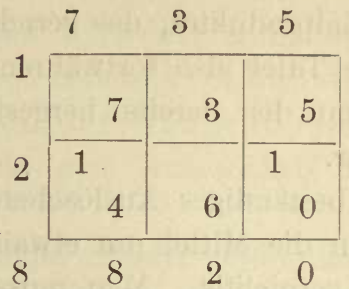

Die Produkte der einzelnen Ziffern sind derartig in Einer und Zehner zerlegt, dass man hinterher nur das Zusammenzählen in der Richtung der einen Diagonale der kleinen Quadrate vorzunehmen hat.

Die an das Positionssystem angeschlossenen Rechenregeln waren bei den Indern der Hauptsache nach dieselben, die noch heutigen Tages benutzt werden. Die Abweichungen davon hatten meist rein äussere Gründe. So hatte man Rechentafeln, die im Verhältnis zu den ziemlich grossen Ziffern, die man der Deutlichkelt wegen schreiben musste, nur klein waren; aber diese Ziffern liessen sich leicht auslöschen und durch andere ersetzen. Aus diesem letzten Grunde stand dem nichts im Wege, von links nach rechts $\mathrm{zu}$ addieren und $\mathrm{zu}$ multiplicieren, wenn man nur beständig die bereits hingeschriebenen Ziffern durch Hinzufügen der überschiessenden Einheiten höherer Ordnung verbesserte. Bei Multiplikation mit einer mehrziffrigen Zahl begann man, wenn man tüchtig genug war um ein so umständliches Aufschreiben wie das eben angeführte entbehren zu können, damit, mit der höchsten Ziffer zu multiplicieren. Während man mit der nächsthöchsten multiplicierte, konnte man gleichzeitig das dadurch entstandene Partialprodukt zu dem bereits gebildeten addieren und dieses in die so gebildete Summe umändern u. s. w. Ausser Multiplikator und Multiplikandus, welcher letztere beständig so verschoben wurde, dass seine Einer ebensoweit nach rechts standen, wie 
die Einer des Partialproduktes, das gerade gebildet werden sollte, enthielt die Tafel also fortwährend nur eine Zahl, die durch Addition der bereits hergestellten Partialprodukte gebildet war.

Ein solches beständiges Auslöschen verlangt grosse Sicherheit, da man die Mittel um etwaige Fehler zu entdecken beständig vernichtet. Man muss namentlich darauf bauen können, dass das Gedächtnis sowohl die augenblicklich vorkomnenden Zahlen, als auch die Tabellen, die benutzt werden, festhält. Solche Tabellen werden heutigen Tages in Indien in grossem Umfange auswendig gelernt, und in alten Zeiten ist es sicher nicht weniger der Fall gewesen. Man lernt jetzt Multiplikationstabellen auswendig, deren einer Faktor eine von den Zahlen von 1 bis 10 ist, der andere eine von den Zahlen von 1 bis 30 , ja bis 100 , nebst den Brüchen $\frac{1}{4}, \frac{1}{2}, \frac{3}{4}, 1 \frac{1}{2}, 2 \frac{1}{2}, 3 \frac{1}{2}$, ferner umfassende Quadrattafeln. Bei einem derartig geübten Gedächtnis ist es nicht zu verwundern, wenn die Inder auch imstande waren die jetzt sogenannte Fouriersche Multiplikation grösserer Zahlen- anzuwenden, die darin besteht sofort (kreuzweise) die Produkte von den einzelnen Ziffern der Faktoren, welche dieselbe decimale Einheit zum Produkte haben, zu bilden und zu addieren.

\section{Anwendungen des Zahlenrechnens.}

Wir wollen nun sehen, auf welche Aufgaben die Inder ferner die numerische Rechenfertigkeit, für welche die Bildung des Positionssystem das beste Zeugnis ist, anzuwenden vermochten, und wofür dieses System demnächst das beste Hülfsmittel wurde. Aufklärungen darüber können wir namentlich in den zahlreichen Rechenregeln und der reichen Sammlung von Aufgaben finden, 
die in Bhâskaras Lîlâvatî enthalten sind, aber auch anderswo. So giebt bereits Âryabhatta dieselben Regeln für das Ausziehen der Quadrat- und Kubikwurzel, die wir jetzt aus den Ausdrücken für $(a+b)^{2}$ und $(a+b)^{3}$ ableiten.

Von dem Inhalte unserer gewöhnlichen Rechenbücher kannten die Inder einfache und zusammengesetzte Regula de tri, Zinsrechnung, auch mit Zinseszins, Gesellschaftsrechnung, die Mischungsregel, Regeln für Raummessung u. s. w. Verschiedene andere Aufgaben, die wir jetzt auf eine Gleichung bringen, werden auch nach bestimmten Rechenregeln gelöst. Hierzu gehört die «Regel des falschen Ansatzes» (regula falsi), die wir bei den Ägyptern (S. 11) gefunden haben; bei dieser bleiben sie jedoch nicht stehen. Aus späteren arabischen Quellen wissen wir, dass sie auch die sogenannte "Regel der zwei falschen Ansätze» (regula duorum falsorum) benutzten. Diese dient dazu eine Aufgabe, die - wenn man sie auf eine Gleichung gebracht hätte -- von einer Gleichung ersten Grades von der Form

$$
f(x)=a x+b=k
$$

abhängen würde, durch zwei Versuche zu lösen. Geben $x=\alpha$ und $x=\beta$ beim Einsetzen in die linke Seite die von $k$ verschiedenen Werte $f(\alpha)$ und $f(\beta)$, so wird $x$ aus den Abweichungen $k-f(\alpha)$ und $k-f(\beta)$ nebst $\alpha$ und $\beta$ nach einer Rechenregel bestimmt, die sich durch die Formel

$$
x=\frac{\beta[k-f(a)]-\alpha[k-f(\beta)]}{[k-f(\alpha)]-[k-f(\beta)]}
$$

wiedergeben lässt. Wie man sieht fällt diese Rechenregel genau mit dem zusammen, was wir jetzt einfache Interpolation nennen, und wir wissen dann, dass sie 
sich nicht nur wie bei den Indern für exakte Berechnung verwenden lässt, wenn $f(x)$ wirklich eine ganze Funktion vom ersten Grade wird, sondern auch für weitergehende Annäherung, wenn $\alpha$ und $\beta$ bereits Näherungswerte sind. Eine derartige Anwendung treffen wir jedoch erst später bei den Arabern und in Europa.

Eine andere Rechenregel von sehr allgemeiner Beschaffenheit ist die Regel der Umkehrung. Diese besteht ganz einfach darin, dass man, wenn man eine Zahl finden soll, die, nachdem sie einer gewissen Reihe von Rechnungen unterworfen ist, eine bekannte Zahl darstellt, dies dadurch erreicht, dass man die letztgenannte Zahl allen umgekehrten Rechnungen in umgekehrter Reihenfolge unterwirft.

Im übrigen werden verschiedene specielle Regeln aufgestellt, die wir durch Auflösen von Gleichungen ersten oder zweiten Grades mit einer oder mehreren Unbekannten finden würden, und die die Inder, wie wir bald sehen werden, ebenso hätten finden können. Das gilt z. B. von den Rechenregeln über Differenz- und Quotientenreihen, bei denen nicht allgemeine Relationen aufgestellt werden, in denen man die verschiedenen Grössen als Unbekannte betrachten kann, sondern bei denen besondere Regeln mitgeteilt werden um jede einzelne von den Grössen zu berechnen, wenn die übrigen bekannt sind. In Lîlâvatî werden sie ohne Beweis mitgeteilt. Dasselbe gilt von verschiedenen anderen Regeln, die wir lieber im nächsten Abschnitt als Zeichen für die zahlentheoretischen Kenntnisse der Inder mitnehmen wollen.

Dagegen dürfen wir die Rechenkunst der Inder nicht verlassen ohne einige Proben von den Formen zu geben, in die sie ihre Beispiele für die Anwendung ihrer verschiedenen Rechenregeln kleiden. 
Eine rein numerische Anwendung der erwähnten Methode der Umkehrung ist das folgende Beispiel:

Schönes Mädchen mit den funkelnden Augen! (das ist Lîlâvatî). Du, die $\mathrm{Du}$ die richtige Methode der Umkehrung kennst! Nenne mir die Zahl, die mit 3 multipliciert, um $\frac{3}{4}$ des Produktes vermehrt, dividiert durch 7, vermindert um $\frac{1}{3}$ des Quotienten, multipliciert mit sich selbst, vermindert um 52, durch Ausziehen der Quadratwurzel, Addition von 8 und Division mit 10 eine 2 giebt.

Eine Aufgabe die sich durch die einfache Regel des falschen Ansatzes lösen lässt, ist folgende:

Ein fünftel eines Bienenschwarms setzt sich auf eine Kadambablume, ein Drittel auf eine Silindhablume, das Dreifache von der Differenz zwischen diesen beiden Zahlen ist auf eine Kutajablume geflogen, und eine einzelne Biene ist in der Luft umhergeflogen, angezogen vom Dufte eines Jasmins und eines Pandanus. Nenne mir, schönes Mädchen, die Anzahl der Bienen!

Eine quadratische Gleichung wird in folgender Gestalt dargestellt:

Mitten im Kampfe ergriff Prit'has rasender Sohn eine Anzahl Pfeile um Carna zu töten. Die Hälfte gebrauchte er zu seiner eigenen Verteidigung und das Vierfache der Quadratwurzel gegen die Pferde. 6 Pfeile durchbohrten den Kutscher Salya, 3 andere spalteten den Sonnenschirm und zerbrachen die Standarte und den Bogen, und Carnas Haupt wurde von einem Pfeil durchbohrt. Wie viele Pfeile hatte Arjuna (Prit'has Sohn)?

Das folgende soll, was nicht leicht zu wissen ist, ein Beispiel für umgekehrte Regula de tri sein:

Wenn eine Sklavin von 16 Jahren 32 Nishkas kostet, was kostet dann eine von 20 Jahren?

Als Unterlage für diese Berechnung wird nur ange- 
führt, das der Wert lebender Geschöpfe sich nach dem Alter richtet.

Etwas nüchterner sind folgende Beispiele für zusammengesetzte Regula de tri:

30 Balken von 12 und 16 Zoll Dicke und Breite und 14 Fuss Länge kosten 100 Nishkas; was kosten dann 14 Balken von 8 und 12 Zoll Dicke und Breite und 10 Fuss Länge? Wenn es 8 Drachmen kostet die ersten Balken eine Meile fortzuschaffen, wie viel kostet es dann die letzten Balken 6 Meilen fortzuschaffen?

Selbst hier würde es in der Praxis nicht immer aus. gemacht sein, ob Proportionalität stattfindet zwischen Preis und Grösse des Balkens oder der Menge von dem, was fortgeschafft werden soll. Man kann also durchgehends sagen, dass die Beispiele aus Interesse und zur Einübung der Berechnung gemacht seien.

Diese wenigen Beispiele zeigen, wie verschieden die Gebiete sind, denen die Aufgaben entnommen werden; bei anderen werden bald eine Anzahl von Blumen, bald ein Zinsfuss, bald eine geometrische Grösse gesucht. Gerade die reichen Einkleidungen verraten die Freude, die man am Aufstellen und Lösen von Aufgaben hatte. In Übereinstimmung hiermit schliesst ein Schriftsteller des 7 ten Jahrhunderts seine Arbeit mit folgenden Worten: "Wie die Sonne durch ihren Glanz die Sterne übertrifft, so wird der Einsichtsvolle den Ruhm anderer verdunkeln, wenn er in der Volksversammlung algebraische Aufgaben vorlegt, und noch mehr wenn er sie löst.»

\section{Algebra und Zahlentheorie; Geometrie.}

Wir wenden uns nun zu der Algebra, die bei Bhâskara namentlich in seiner Vîjaganita oder 
Wurzelberechnung behandelt wird, von der man sagen kann, dass sie ein mit Beweisen verbundenes Rechnen sei. Diese Beweise werden jedoch keineswegs mit griechischer Strenge geführt und bestehen im wesentlichen darin, dass die Aufgaben hier auf eine Gleichung gebracht sind, deren Lösung auch in der That eine Begründung für die Richtigkeit der Rechnungen giebt, die zur Auflösung führen. Dadurch erfährt man teilweise, wie die in Lîlâvatî gegebenen Rechenregeln gefunden sein können.

Die indische Algebra stimmt mit derjenigen Diophants darin überein, dass sie sich von der geometrischen Darstellung frei gemacht hat und die Zahlen nur als solche behandelt. Für Diophant als Griechen ergab sich indessen hieraus die Forderung, dass die Grössen, die aus der Rechnung hervorgingen, Zahlen, d. h. rationale Zahlen sein sollten. Wenn auch die Inder wegen ihrer weniger feinfühligen Logik kein Bedenken trugen ohne weiteres die Rechenregeln von rationalen auf irrationale Zahlen zu übertragen, so gab dennoch eben dieser Umstand ihren Operationen einen weit grösseren Umfang. Statt der in geometrischer Form gehaltenen Umformungen irrationaler Grössen bei Euklid treffen wir daher bei den Indern das Rechnen mit irrationalen Zahlen. Sie kannten Regeln um Brüchen rationale Nenner zu geben und - was Euklid in geometrischer Form ausführt um doppelte Irrationalität aufzuheben. Sie wussten sogar, dass

$$
\begin{gathered}
\sqrt{16+\sqrt{120}+\sqrt{72}+\sqrt{60}+\sqrt{48}+\sqrt{40}+\sqrt{24}} \\
=\sqrt{2}+\sqrt{3}+\sqrt{5}+\sqrt{6}
\end{gathered}
$$

ein Beispiel, das jedoch wahrscheinlich durch die umgekehrte Rechnung gebildet worden ist. 
Auch in anderer Beziehung überschritten die Inder die Grenzen, die ein vorsichtiger Grieche sich setzen musste. Da die Griechen durchaus nicht den Begriff "negative Grössen» aufgestellt hatten, so mussten sie dafür sorgen, dass die Grössen, die gleich gesetzt wurden, sicher positiv waren, und wenn eine gestellte Aufgabe von selbst zu einem negativen Resultat führte, so musste auch der Grieche, der die Bedeutung hiervon erkannte, sofort diese Erkenntnis benutzen um die Form der Aufgabe so zu verändern, dass nach der entsprechenden positiven Grösse gefragt wurde. Die rechnenden Inder nahmen die Rechnungen und ihre Resultate mehr so, wie sie sich von selbst darboten. Sie kümmerten sich nicht darum, in wie weit eine Grösse auf der einen Seite des Gleichheitszeichen wirklich positiv oder negativ war, und wenn eben die gesuchte Grösse negativ wurde, so haben sie allerdings oft eine solche Wurzel verworfen, oft aber auch verstanden sich dadurch mit ihr abzufinden, dass sie sie als Schuld bezeichneten. Sie haben auch, wenn auch zunächst nur zur Benutzung bei Behandlung der einzelnen Glieder in Rechnungen mit mehrgliedrigen Grössen, Regeln aufgestellt für das\$ Rechnen mit Grössen, die mit Vorzeichen versehen sind. In Verbindung hiermit erkannte man, das eine Quadratwurzel mit doppeltem Vorzeichen gerechnet werden muss, und deshalb legte man einer Gleichung zweiten Grades zwei Wurzeln bei. Wurde die eine von diesen negativ, so wurde sie jedoch in der Regel verworfen.

Ein anderer Beweis für eine glückliche Deutung ist eine vorkommende richtige Erklärung von $\frac{a}{0}$. Indessen trifft man auch auf ganz unrichtige Anwendungen von derartig gebildeten Grössen. 
Was die analytischen Hülfsmittel der Inder betrifft, so benutzten sie wie Diophant Zeichen, die in Wirklichkeit Abkürzungen von Wörtern waren, um gesuchte Grössen und ihre Potenzen darzustellen; sie gingen aber weiter als jener, da sie gleichzeitig mehrere verschiedene Unbekannte bezeichnen konnten. Das thaten sie, indem sie jeder von diesen eine verschiedene Farbe beilegten, deren Namen sie dann auch abkürzten. Diese Erweiterung der Zeichensprache gab auch Veranlassung zur Erweiterung der Rechnungen mit den Grössen, die durch diese Zeichensprache dargestellt wurden.

Diese verbesserten Hülfsmittel erwiesen sich den Indern nützlich bei ihrer Behandlung von bestimmten Gleichungen mit einer oder mehreren Unbekannten; aber auf diesem Gehiete treffen wir dennoch nichts anderes als das, was auch die Griechen - denen sie gewiss die Lösung der Gleichungen zweiten Grades verdankten behandeln konnten. Etwas Neues treffen wir dagegen auf dem Gebiete der unbestimmten Gleichungen. Dieses Neue besteht namentlich darin, dass die Inder sich hier nicht wie Diophant mit rationalen Lösungen begnügten, sondern Lösungen in ganzen Zahlen verlangten.

Dadurch erhalten sie bereits Gelegenheit sich mit unbestimmten Gleichungen ersten Grades zu beschäftigen. Um eine solche Gleichung durch ganze Zahlen zu lösen, benutzten die Inder ungefähr dieselben Rechnungen, die entstehen, wenn man jetzt die Aufgabe durch Kettenbrüche löst. Da die Regeln ohne Beweis gegeben werden, so wissen wir nicht, wie sie gefunden sind, und wollen deshalb nur bemerken, dass man leicht zu ihnen gelangen kann, ohne solche Begriffe, wie Kettenbrüche und ihre Näherungswerte aufzustellen. Zunächst 
ist es einleuchtend, dass man durch Multiplikation mit $c$ die Lösungen der Gleichung

$$
a x-b y=c
$$

aus den Lösungen der Gleichung

$$
a x-b y=1
$$

ableiten kann. Ist in dieser letzten Gleichung $a>b$, und erhält man bei der Division von $a$ durch $b$ den Quotienten $q$ und den Rest $r$, so wird

$$
y=q x+\frac{r x-1}{b}
$$

eine solche Bestimmung von $x$, dass $\frac{r x-1}{b}=z$ eine ganze Zahl wird, hängt dann von einer Gleichung mit einfacheren Koefficienten ab. Bei der Reduktion kommen genau dieselben Zahlen vor, wie wenn man das grösste gemeinschaftliche Maass sucht, und das Verfahren ist fortzusetzen, bis man zu einem Koefficienten 1 gelangt. Das Einsetzen hinterher fällt zusammen mit der Berechnung der Näherungswerte eines Kettenbruches.

Indessen beschäftigte man sich nicht nur mit einer einzigen Gleichung mit zwei Unbekannten, sondern auch nit Gleichungen mit mehr Unbekannten. Die Aufgaben gehen oft darauf aus eine Zahl zu finden, die, durch verschiedenen gegebenen Zahlen dividiert, gegebene Reste giebt. Möglicherweise rühren diese Aufgaben ursprünglich von den Chinesen her, bei denen man eine alte Regel für ihre Auflösung gefunden hat. Oft betreffen sie die Bestimmung der astronomischen Perioden, nach denen eine Gruppe von Erscheinungen aufs neue zusammentrifft, was Veranlassung zu Verfinsterungen u. s. w. geben kann. Die Längen eben dieser Perioden, die den griechischen Astronomen bekannt waren, geben wohl noch 
nur Veranlassung $\mathrm{zu}$ homogenen Gleichungen. Wenn z. B. nach der Zeit gefragt wird, die sowohl eine ganze Anzahl von Tagen, $x$, als auch eine ganze Anzahl von Jahren, $t$, enthält, wenn 30 Jahre $=10960$ Tage, so wird

$$
10960 t=30 x \text { oder } \frac{x}{10960}=\frac{t}{30} \text {. }
$$

Fragt man dagegen, wann das in Rede stehende Zusammentreffen wirklich eintritt, so erhält man vollständige unbestimmte Gleichungen ersten Grades. Fehlen beispielsweise $\frac{m}{n}$ Tage, bis der Tag zu Ende ist, und $\frac{p}{q}$ Jahre, bis das Jahr zu Ende ist, und soll der Augenblick, wo Tages- und Jahreswechsel zusammenfallen, nach $\left(x+\frac{m}{n}\right)$ Tagen $=\left(t+\frac{p}{q}\right)$ Jahren eintreffen, so mnss man haben

$$
10960\left(t+\frac{p}{q}\right)=30\left(x+\frac{m}{n}\right) .
$$

Bhâskara vereinfacht jedoch die Frage durch die Annahme, die sich immer mit einer gewissen Annäherung erreichen lässt, dass in den Brüchen die Nenner $q=10960$ und $n=30$ sind.

Die Gleichung $x y+-a x+b y=c$ wird leicht dadurch gelöst, dass man sie in

$$
(x+b)(y+a)=c+a b
$$

umformt, worauf es nur darauf ankommt, $c+a b$ in ein Produkt von ganzen Faktoren zu zerlegen.

Grössere Schwierigkeiten haben die Inder überwunden bei der Behandlung von unbestinmten Gleichungen, die mit Bezug auf jede der Unbekannte vom zweiten Grade sind. Von diesen suchen sie nicht nur wie Diophant, 
durch.den sie gleichwohl in diese Fragen eingeführt sein können, rationale, sondern ganze Auflösungen. Namentlich beschäftigten sie sich mit Gleichungen von der Form

$$
y^{2}=a x^{2}+b,
$$

auf die sich auch andere unbestimmte Gleichungen zweiten Grades reducieren lassen. Um eine Vorstellung von der Art und Weise ihrer Behandlung zu geben, will ich hier ihre Lösung der besonders wichtigen Gleichung

$$
y^{2}=a x^{2}+1
$$

mitteilen.

Wir beginnen mit einem Verfahren, das von demjenigen Diophants verschieden ist, und sich zur Bildung einer unbegrenzten Anzahl rationaler Lösungen benutzen lässt. Man bildet zuerst die Gleichungen

$$
\left.\begin{array}{l}
a x_{1}{ }^{2}+b_{1}=y_{1}{ }^{2} \\
a x_{2}{ }^{2}+b_{2}=y_{2}{ }^{2}
\end{array}\right\}
$$

aus denen sich $b_{1}$ und $b_{2}$ bestimmen lassen, wenn man $x_{1}, y_{1}, x_{2}$ und $y_{2}$ beliebig wählt. Reduciert man die Gleichungen auf $b_{1}$ und $b_{2}$ und multipliciert sie mit einander, so erhält man

$$
\left(a x_{1} x_{2}+y_{1} y_{2}\right)^{2}-a\left(x_{1} y_{2}+x_{2} y_{1}\right)^{2}=b_{1} b_{2}
$$

also eine dritte Gleichung

worin

$$
\left.\begin{array}{c}
a x_{3}{ }^{2}+b_{3}=y_{3}{ }^{2} \\
b_{3}=b_{1} b_{2}, x_{3}=x_{1} y_{2}+x_{2} y_{1} \\
y_{3}=a x_{1} x_{2}+y_{1} y_{2}
\end{array}\right\}
$$

Lässt man die beiden Gleichungen (3) identisch sein, so ergiebt sich 
4. Algebra und Zahlentheorie; Geometrie.

oder

$$
a\left(2 x_{1} y_{1}\right)^{2}+b^{2}=\left(a x_{1}^{2}+y_{1}^{2}\right)^{2}
$$

$$
a\left(\frac{2 x_{1}}{b} y_{1}\right)^{2}+1=\left(\frac{a x_{1}^{2}+y_{1}^{2}}{b}\right)^{2}
$$

und damit hat man eine rationale Lösung der Gleichung (2). Sucht man nun dadurch weiter zu kommen, dass man für $x_{1}$ und $y_{1}$ willkürlich gewählte Werte einsetzt, so kann man oft erreichen, dass die gefundenen Werte von $x$ und $y$ ganze Zahlen werden. Im besonderen sind die Fälle zu beachten, in denen man bereits erreicht hat, dass $b= \pm 1$ oder \pm 2 .

Ist $b=1$, so kann man auf diesem Wege aus einer Lösung von (2) eine neue ableiten, und darauf so viele wie man will.

Ist $b=-1$ oder \pm 2 , so wird (5) auch eine Lösung von (2) in ganzen Zahlen geben; denn für $b= \pm 2$ ist $y_{1}{ }^{2}=a x_{1}^{2}+2$, also wird $a x_{1}{ }^{2}+y_{1}{ }^{2}=2 a x_{1}{ }^{2} \pm 2$ gerade. Zugleich ergiebt sich aus (4), dass die Kenntnis einer Lösung von (2) gestattet, aus einer Lösung von (1) unendlich viele Lösungen abzuleiten.

Gelingt es nun nicht für einen gegebenen Wert von a durch Versuche irgend eine Gleichung von der Form (1) zu bilden, wo $b= \pm 1$ oder \pm 2 , so benutzt man die sogenannte cyklische Methode um den Wert von $b$ zu reducieren.

Es sei

$$
a x_{1}{ }^{2}+b_{1}=y_{1}^{2}
$$

eine Gleichung, in der $b_{1}$ bereits so klein ist, wie man es durch Versuche erreichen kann, die darin bestehen können, dass man $\frac{y_{1}}{x_{1}}$ einen Näherungswert von $\sqrt{a}$ sein lässt. $x_{1}$ und $b_{1}$ enthalten dann keinen gemeinsamen Faktor, denn ein solcher würde quadratischer Faktor auf 
beiclen Seiten des Gleichheitszeichen sein, und durch Verkürzung würde man eine einfachere Gleichung derselben Art erhalten. Man setzt nun

$$
\frac{x_{1} z+y_{1}}{b_{1}}=x_{2},
$$

woraus sich $x_{2}$ und $z$ als ganze Zahlen bestimmen lassen. Man wählt diejenigen, die $z^{2}-a$ so klein wie möglich machen. Setzt man dann $\frac{z^{2}-a}{b_{1}}=b_{2}$, so ist einmal $b_{2}$ eine ganze Zahl, und zweitens $a x_{2}{ }^{2}+b_{2}$ eine neue Quadratzahl $y_{2}{ }^{2}$. Diese Dinge lassen sich leicht beweisen, aber die indischen Schriftsteller beweisen weder dieses, noch dass man wirklich auf diese Weise zu $b=1$ gelangen kann. Das letztere, zu dessen theoretischer Begründung die Inder sicher die erforderliche mathematische Einsicht nicht besassen, hat erst Lagrange, der selbst dieselbe Lösung wiedergefunden hat, bewiesen. Indessen hat die grosse Zahlenfertigkeit der Inder sich dadurch $\mathrm{zu}$ erkennen gegeben, dass ihre numerischen Versuche sie zu einer vollkommen richtigen Methode geführt und dahin gebracht haben, durch Anwendungen Vertrauen zu ihrer allgemeinen Brauchbarkeit zu gewinnen.

Neben solchen zahlentheoretischen Methoden wie die im Vorhergehenden geschilderte besassen die Inder verschiedene zahlentheoretische Sätze, unter diesen z. B. den folgenden: Die Grössen

$$
\left(\frac{\left(\frac{8 p^{2}-1}{2 p}\right)^{2}}{2}+1\right)^{2} \pm\left(\frac{8 p^{2}-1}{2 p}\right)^{2}-1
$$

sind beide Quadrate. Wir wollen hier gleichfalls anführen, das die Inder die Formeln für die Anzahl der Permuta- 
tionen und Kombinationen, und, wie die Griechen, für die Summen der Quadrate und Kuben der ersten Zahlen der Zahlenreihe kannten und anwendeten.

Bei der Geometrie der Inder länger $\mathrm{zu}$ verweilen bietet sich keine Veranlassung. Die meisten von den Sätzen, die sie kannten, verdanken sie gewiss den Griechen, aber die auf diesen aufgebaute Berechnung trieben sie oft weiter, als diese gethan haben. Ein Satz bei Brahmagupta hat ein gewisses Aufsehen erregt, nämlich eine Erweiterung von Heros Dreiecksformel auf Vierecke. So wie der Satz dasteht, sieht er aus, als ob der Inhalt eines beliebigen Vierecks durch $\sqrt{(s-a)(s-b)(s-c)(s-d)}$ dargestellt werden solle, worin $a, b, c$ und $d$ die Seiten, und $s$ ihre halbe Summe bedeutet. Bereits Bhâskara nahm die Sache so, und hält sich dann mit Recht über den Fehler auf, der in der Annahme liegt, dass ein Viereck durch seine Seiten bestimmt sein sollte. In Wirklichkeit beschäftigt Brahmagupta sich jedoch nur mit zwei bestimmten Klassen von einbeschriebenen Vierecken, für welche der Satz ja richtig ist, aber es ist möglich, dass er sich von dieser Begrenzung, die bei der Angabe der Resultate nicht ausgesprochen wird, keine Rechenschaft abgelegt hat. Die Klassen von Vierecken, die er behandelt sind teils gleichschenkelige Trapeze, teils einbeschriebene Vierecke mit senkrecht auf einander stehenden Diagonalen. Eine Veranlassung, die indessen nicht bei Bramagupta hervortritt, können die Inder gehabt haben sich mit den letztgenannten Vierecken zu beschäftigen, nämlich ihre Trigonometrie, in der sie nicht wie Ptolemäus Sehnentafeln sondern Sinustafeln benutzten. Ist nämlich der Durchmesser des Kreises gleich 1, und betragen zwei zusammenstossende Bogen $2 x$ und $2 y$, so sieht man, dass die Seiten des Vierecks $\sin x, \sin y, \cos x$ und $\cos y$ sind, und dass die Diagonalen sich beziehungsweise in die 
Abschnitte $\sin x \cos y$ und $\sin y \cos x$, und in die Abschnitte $\sin x \sin y$ und $\cos x \cos y$ teilen. Die Figur ist also wahrscheinlich diejenige gewesen, die man zur Bestimmung von $\sin (x+y)$ benutzt hat.

Die Sinustafeln, die sich in Sûrya Siddhânta finden, gehen jedoch nur hinunter zu Intervallen von $3 \frac{3}{4}^{0}$, während die Sehnentafeln des Ptolemäus Sinustafeln mit einem Intervall von $\frac{1}{4}^{0}$ entsprechen. Sind diese Tafeln - wie so vieles andere vom Inhalte dieses Buches - griechischen Ursprunges, so müssen sie aus Werken herrühren, die älter sind als diejenigen des Ptolemäus; dann stammen sie vielleicht von den Astronomen in Alexandria, die im Gegensatz zu Hipparch und seiner Schule Sinustafeln gebraucht haben können. Ebendaher kann vielleicht der Näherungswert $\frac{31416}{10000}$ für $\pi$, der sich bei Âryabhatta findet, gekommen sein, da wir wissen, dass Apollonius $\pi$ genauer bestimmte als Archimedes. Dagegen deutet die willkürliche Annäherung $\pi=\sqrt{10}$, die sich bei Brahmagupta findet, nicht auf griechischen Ursprung, und ebensowenig finden wir griechische Vorbilder für eine Näherungsformel zur Berechnung der Sehne $k$ eines gegebenen Bogens, die bei Bhâskara vorkommt, nämlich

$$
k=4 d \frac{b(p-b)}{\frac{5}{4} p^{2}-b(p-b)}
$$

worin $d$ den Durchmesser, $p$ die Peripherie und $b$ die Länge des Bogens bedeutet. 


\section{Das Mittelalter.}

\section{Allgemeine Einleitung.}

Im Altertum hatten die Griechen, wie wir gesehen haben, eine Geometrie aufgebaut, welche die Raumverhältnisse mit einer solchen Vollständigkeit und mit solcher Sicherheit in allen Schlüssen behandelte, dass sie vollauf ihren Platz als Wissenschaft selbst gegenüber den strengsten Anforderungen der neuesten Zeit behaupten kann. Da die geometrischen Formen zugleich ein Mittel waren zur Darstellung allgemeiner kontinuierlicher Grössen, so enthält diese Geometrie auch einen grossen Teil von dem, was wir jetzt reine Mathematik nennen. Unter dieser Form war die Algebra fortgeführt bis zur Lösung von Gleichungen zweiten Grades und ihren weitergehenden Anwendungen, und bis zu einer Behandlung von Gleichungen dritten Grades, die allerdings nicht die Zurückführung auf Wurzelgrössen enthielt, die wir jetzt die Lösung dieser Gleichungen nennen, die aber durch Anwendung der Lehre von den Kegelschnitten Mittel lieferte zur Diskussion und zu einer theoretischen Untersuchung der Aufgaben, die von diesen Gleichungen abhängen. Dieselbe Methode liess sich auch auf Probleme anwenden, die von Gleichungen vierten Grades abhängen würden, ohne dass jedoch von irgend welcher direkten Darstellung 
solcher Gleichungen die Rede wäre. Neben diesen Aufgaben, die unter die endliche Analysis gehören, hatten die Griechen auch die Behandlung solcher Aufgaben begonnen, die jetzt der Integralrechnung angehören, und wenn auch diese Behandlung nicht dahin gelangte viele einzelne Fragen zu umfassen, so wurde sie doch in Formen vorgenommen, deren grosser wissenschaftlicher Wert in der neueren Zeit um so mehr Anerkennung gewinnt, je höher die wissenschaftlichen Anforderungen steigen. Endlich haben wir gesehen, dass die zu Anfang versäumte numerische Anwendung der Mathematik sich allmählich unter den steigenden Ansprüchen der Astronomie entwickelte, und bei Diophant haben wir Proben von einer eingehenden Untersuchung über die numerischen Bedingungen für Rationalität kennen gelernt.

Auf der anderen Seite haben wir gesehen, dass die alte Fertigkeit der Inder in der Behandlung von Zahlen sich $\mathrm{zu}$ einer wirklichen Rechenkunst entwickelt hatte, die dasselbe Hülfsmittel, das wir jetzt gebrauchen, das Positionssystem, benutzte. Durch Berührung mit der griechischen Mathematik hatte diese Rechenfertigkeit auch bei den Indern eigentliche mathematische Fortschritte herbeigeführt. Der bedeutendste von diesen war eine zweckmässige Behandlung von Fragen, die ganze Zahlen betrafen. Einige von diesen Fortschritten gehören jedoch vielleicht erst einer Zeit an, wo neue mathematische Arbeiten bei den Arabern begonnen waren.

Um die Verdienste der Völker, zu denen die Entwickelung der Mathematik von den Griechen und Indern überging, nach Gebühr würdigen zu können, hat man jedoch zu beachten, wie weit die vorliegende Form der Mathematik davon entfernt war, den neuen Völkern bequem zugänglich zu sein. Bei den späteren Griechen selbst hatten die aufbewahrten Werke allerdings ein Ver- 
ständnis der Einzelheiten aufrecht erhalten oder doch dann und wann wiedererwecken können, aber sie hatten nicht zu einem Überblick geführt, ohne den weitergehende Arbeiten unmöglich werden. Über die Methoden der Arbeit, die ihrerzeit zu so grossen Resultaten geführt hatten, geben diese Werke keine Auskunft, und jede fruchtbare Tradition darüber war längst verloren gegangen. Man musste deshalb selbst diese oder andere Methoden der Arbeit wiederfinden, bevor von einer vollständigen Aneignung des Inhaltes die Rede sein konnte; ja von manchem Resultate hat man - und das auch nicht einmal immer erst erkennen können, dass die Griechen es besessen haben, nachdem man es selbst in einer anderen Form wiedergefunden hatte. Während dieser ganzen Wiederaufführung der Mathematik hat jedoch das, was von den Griechen vorlag oder was man allmählich bei ihnen verstehen lernte, selbstverständlich in ausgezeichneter Weise zur Führung und Anleitung gedient.

Die indische Rechenkunst mochte, wo die Gelegenheit dazu sich darbot, durch ihre praktische Anwendbarkeit etwas grössere Aussicht haben durchzudringen. Indessen hat man zu beachten, dass es auch bei dieser nicht genügt ihre Principien kennen zu lernen, um sie richtig würdigen $\mathrm{zu}$ können. Wir können sie nicht anwenden ohne in unserer Kindheit eine gewisse Arbeit daran gegeben zu haben, um die einfachsten Tabellen auswendig zu lernen und in ihrer Benutzung geübt zu werden. Ihre Vorteile fielen deshalb nicht sogleich in die Augen, vielleicht am wenigsten bei denen, die bereits Arbeit darauf verwendet hatten andere Methoden des Rechnens einzuüben.

Die unmittelbarsten Erben der am Schlusse des Altertums vorliegenden griechischen Mathematik, der einzelnen überlieferten Hauptwerke und des beständig abnehmenden 
Verständnisses von diesen, waren das oströmische Reich und die griechisch-katholische Kultur, die in Konstantinopel dieselbe Hauptstadt hatte wie dieses Reich. Obgleich hierzu später solche Einwirkungen kamen, die sich von Indien herschreiben, so treffen wir in Konstantinopel dennoch im wesentlichen nur eine Fortsetzung des begonnenen Hinsiechens. Das empfangene Pfund, nämlich die noch existierenden Werke der grossen griechischen Geometer, wurden vergraben, ohne irgend welchen Zins zu geben, aber sie liessen sich dann wieder am Schlusse des Mittelalters ausgraben und konnten dann aufs neue der europäischen Kultur zu Gute kommen. Das geschah jedoch erst, nachdem ein Teil der Werke und neues von Verständnis begleitetes Interesse für sie auf anderem Wege nach Westeuropa gekommen war, nämlich durch die Araber.

Ein solches Pfund wurde nicht den Völkerschaften anvertraut, die durch die Völkerwanderung das Über. gewicht in der westeuropäischen Entwickelung erhielten. Diese nahmen das Christentum an und erhielten Anteil an der römischen Kultur, aber zu dieser gehörte die Mathematik nicht. Nachdem die Romantiker ihrerzeit das westeuropäische Mittelalter mit einem in vieler Bezichung gewiss unverdienten Strahlenglanz umgeben haben, ist man in unserer Zeit am meisten geneigt in die entgegengesetzte Äusserlichkeit $\mathrm{zu}$ verfallen und nur von dem finsteren Mittelalter zu sprechen. Auch dieses Urteil dürfte viel unbilliges enthalten, wenn man auf den Kulturstandpunkt Rücksicht nimmt, auf dem die meisten der betreffenden Völkerschaften am Beginn und am Schlusse des Mittelalters standen. Was sie wirklich im Christentum und in römischen Gesetzen und Institutionen empfangen hatten, das wurde namentlich in den Klöstern von fleissigen Männern mit geistigen Interessen verarbeitet 
und gereichte den Völkern durch seinen eigenen Wert und seinen kulturfördernden Einfluss zum Segen. Mathematik dagegen konnte man im wesentlichen nur kennen lernen durch die dürftigen Auszüge aus den praktischen Regeln Heros und der Ägypter, die man den römischen Landmessern verdankte, oder, wenn es hoch kam, durch die Bruchstücke von Euklid oder Nikomachus, welche die mehr theoretischen, aber ebensowenig wissenschaftlichen Nachfolger der Landmesser in Formen überlieferten, die Veranlassung gaben zu solchen Missverständnịssen wie dasjenige, dass figurierte Zahlen Ausdrücke für Flächeninhalt sein sollten. Das Nützlichste und Zugänglichste des von den Römern, und durch diese von den Griechen erhaltenen mathematischen Erbteils, war die Rechentafel, abacus, die - ungewiss wann - dahin verbessert worden war, dass die Marken oder Steine, die auf die verschiedenen Kolumnen der Tafel gelegt wurden, nun nicht mehr gleich waren und durch ihre Anzahl die Anzahl der decimalen Einheiten bezeichneten, zu denen die Kolumnen gehörten, sondern 9 verschiedene Zeichen enthielten, die den Zahlen von 1 bis 9 entsprachen. Alles in allem stand dieses ganze Erbteil jedoch sicher hinter dem zurück, welches die Ägypter ihrerzeit den Griechen hinterlassen hatten.

Dass dennoch sowohl in den Klöstern wie in den südeuropäischen, namentlich den italienischen Handelsstädten Empfänglichkeit für Mathematik vorhanden war, zeigte sich, als sie in besserer Gestalt nach Europa kam. Das geschah durch die Araber, die sich teils ein richtiges Verständnis der griechischen Mathematik erworben und ihr eine neue Entwickelung gegeben hatten, in der sie leichter zugänglich war als in den überlieferten alten griechischen Schriften, teils in reichem Maasse indische Rechenkunst mit ihr verbunden hatten. Es kostete aller- 
dings einige Zeit bevor die Europäer, nachdem sie durch die Kreuzzüge und in Spanien und auf Sicilien mit den Arabern zusammengetroffen waren, sich die Mathematik der Araber, und durch diese einen Teil der griechischen und indischen Mathematik und Rechenkunst angeeignet hatte. Während dieser Aneignung wurde jedoch der Durchbruch für eine neue und rasche Entwickelung der Mathematik vorbereitet, der im 16ten Jahrhundert mit den grossen Fortschritten zusammenfiel, die nach anderen Richtungen hin den Beginn der neueren $\mathrm{Z}$ eit bezeichnen.

Aus dem hier Gesagten geht hervor, dass der früheste und bedeutendste Teil der mathematischen Entwickelung im Mittelalter bei den Arabern vor sich ging. Was die äusseren Bedingungen für diese Entwickelung betrifft, so möchte ich zuerst auf die ungeheure Geschwindigkeit aufmerksam machen, mit der die Eroberungen der Araber und demnächst der Muhamedanismus sich über weitgestreckte Länder verbreiteten. Die ursprünglichen Bewohner von diesen wurden durch die Annahme dieser Religion bald den Arabern gleich gerechnet, und die vielen Länder wurden dadurch in Verbindung mit einander gebracht. $\mathrm{Zu}$ diesen Ländern gehörte Ägypten, die alte Wiege der Geometrie, mit Alexandrien, wo die Geometrie später zu ihrer höchsten Blüte gelangte und am längsten fortfuhr wirkliche Lebenszeichen von sich $\mathrm{zu}$ geben. $\mathrm{Zu}$ diesen gehörten auch andere Länder, die von Griechen bewohnt oder von griechischer Kultur beeinflusst waren. Die Araber überschwemmten auch die Gegenden, die vor Zeiten der Sitz der babylonischen und chaldäischen Astronomen gewesen waren. Ferner drangen sie bis nach Indien vor und kamen dadurch in genauere Berührung mit der indischen Rechenkunst, als die Griechen es gewesen waren.

Dass sich auf diese Weise günstige Gelegenheiten 
darboten um sich die ganze damals existierende Mathematik anzueignen, genügte jedoch noch nicht. Wir haben ja gesehen, dass die griechische Wissenschaft lange ein toter Schatz für die Griechen selbst gewesen war, und dass die Römer, die dieselbe Gelegenheit gehabt hatten wie jetzt die Araber um der griechischen Mathematik teilhaftig zu werden, und zwar zu einer Zeit, wo diese nur noch wenig von ihrer ursprünglichen Frische verloren hatte, nicht verstanden hatten diese Gelegenheit zu benutzen. Vielleicht war die eigentümliche römische Kultur trotz ihrer Begrenzung zu bedeutend, als dass die Römer mit Bezug auf die schwierigeren Teilen der griechischen Wissenschaft, namentlich auf die Mathematik, so taugliche Schüler werden konnten, wie es einerseits die Völker der Völkerwanderung mit Bezug auf die Kultur wurden, die sie durch die Römer empfangen konnten, und wie sich andererseits nun ein in der Kultur so neues Volk wie die Araber gegenüber den Resten der griechischen Kultur zeigte.

Nicht alle arabischen Herrscher zeigten sich gegen die Wissenschaft so feindlich wie ${ }^{c} \mathrm{Omar}$, der zweite Nazhfolger des Propheten, es war, selbst wenn auch die wesentlichste Zerstörung der Bibliothek in Alexandria vor seiner und der Araber Zeit stattgefunden hat. Im Gegenteil entstanden $\mathrm{zu}$ verschiedenen Zeiten und an verschiedenen Orten Reihen von Fürsten, die eine Ehre darin setzten die Wissenschaft zu fördern, und eben dadurch glaubten ihr eigenes Ansehen zu vermehren. Von diesen soll hier nur die Reihe von Kalifen, die Abbasiden, nämlich Almansûr, Hârûn Arraschid und Almamûn (754-833) genannt werden, die nach ${ }^{c} \mathrm{O}$ mars Geschlecht folgten und 762 Bagdad gründeten und zu ihrem Sitz erhoben. Unter diesen wurden namentlich die Elemente des Euklid und der "Almagest» des Ptolemäus ins 
Arabische übersetzt. Später kamen hierzu Ǔbersetzungen von Diophant, Hero, Archimedes und Apollonius. Ausser diesen Hauptwerken haben die Araber ein nunmehr verloren gegangenes Werk von Hipparch über quadratische Gleichungen gekannt. Gleichfalls lagen frühzeitig Übersetzungen von indischen Schriftstellern vor, und die indische Rechenkunst fand teils durch die indische Astronomie, teils wohl durch die Handelsverbindungen Eingang bei den Arabern. Die eigentlichen mathematischen Fortschritte, die man den Indern verdankt, scheinen dagegen keinen Einfluss auf die Araber geübt zu haben, die mit Recht zu den Griechen als ihren besten Lehrmeistern in wissenschaftlicher Behandlung hinauf sahen und die weniger vollständig begründeten Theorien, die sich von den Indern entnehmen liessen, übersehen hatten. Dass die Übersetzerarbeit sich allmählich bis zu den schwierigsten griechischen Werken entstreckte, ist ein Zeugnis dafür, dass nach und nach die Entwickelung erreicht war, die erforderlich ist um diese zu würdigen und $\mathrm{zu}$ verstehen. Aus dem, was wir hierüber schon früher gesagt haben, geht hervor, dass dies nicht ohne eine bedeutende selbständige Arbeit bei den Arabern erreicht werden konnte. Für den Umfang dieser Arbeit, und für den Ernst, mit dem die einzelnen Mathematiker sich zur Teilnahme daran vorbereiteten, haben wir ein Zeugnis, wenn berichtet wird, dass Diophants Übersetzer A bûl Wafâ, dessen eigene Verdienste wir später erwähnen werden, in seiner Jugend Unterricht in spekulativer und praktischer Arithmetik (d. h. Algebra und Arithmetik) bei zwei Lehrern, und in Geometrie bei zwei anderen Lehrern empfing. 
2. Die Arithmetik und Algebra der Araber.

\section{Die Arithmetik und Algebra der Araber.}

Es war mein Wunsch - unter anderem durch Vergleichung mit den Römern - den Wert und Umfang der mathematischen Arbeiten der Araber hervorzuheben, damit man nicht herabsetzende Schlüsse aus den verhältnismässig wenigen positiven Resultaten ziehe, die sie über das hinaus, was die Griechen bereits wussten, erreicht haben. Dieser letzte Umstand ist indessen ein Grund dafür, dass wir uns hier nicht so viel mit den Arabern beschäftigen können, wie der Umfang ihrer Arbeiten sonst beanspruchen zu können scheint. Freilich werden wir dazu kommen die bedeutendsten mathematischen Schriftsteller anzuführen, aber mehr als Beispiele für die Richtungen, nach denen hin die Araber im ganzen arbeiteten, als als Glieder in einer zusammenhängenden Darstellung der arabischen Mathematik und ihrer Entwickelung.

Die Zeit liegt indessen noch nicht so weit hinter uns, wo man, aus Mangel an richtiger Auffassung von den überlieferten griechischen Schriftstellern und wegen ungenügender Bekanntschaft mit der indischen Mathematik, den Arabern die Ehre für die von den Griechen begründete Algebra sowohl wie für die Rechenkunst der Inder zuerteilte. Diese Vorstellung hat sogar einen bleibenden Ausdruck in dem Namen Algebra erhalten und in einer anderen mathematischen Benennung, Algorithmus, die lange Zeit von der an das Positionssystem angeschlossenen Zahlenrechnung gebraucht wurde, aber jetzt dahin erweitert ist, dass sie von jedem System von Bezeichnungen und Annahmen gebraucht wird, das ein mechanisches Ausrechnen nach bestimmten Regeln gestattet. Beide diese Benennungen rühren von einem einzelnen Mann und von einem seiner Werke her, und mit ihrer 
Benutzung ist die Vorstellung verbunden gewesen, dass wir ihm erst die Algebra und die jetzt gebräuchliche Zahlenrechnung $\mathrm{zu}$ verdanken haben.

Dieser Mann war Muhammed ibn Mûsâ Alchwarizmî, und er gehörte zu dem Kreise von Gelehrten, die der Kalif Almamûn mit Übersetzungen, mit einer geographischen Gradmessung und mit anderen wissenschaftlichen Arbeiten beschäftigte. Das Wort Algorithmus ist gebildet durch Übertragung seines Zunamens auf ein Werk von ihm über die Rechenkunst, in dem Regeln für das Rechnen mit Zahlen, die nach dem Positionssystem geschrieben sind, gegeben werden, ist dann weiter auf Werke übertragen worden, die später die indische Art zu rechnen in Europa verbreitet haben, und von da aus endlich auf diese Art zu rechnen selbst. Das angeführte Werk kennt man durch eine lateinische Übersetzung, die mit den Worten "Dixit Algorithmi» beginnt, und worin Erklärungen von der Zahlenschreibung und den vier Species in ganzen Zahlen und einfachen Brüchen gegeben werden. Verdoppelung und Halbierung werden als besondere Rechnungen aufgestellt. Für die drei ersten Rechnungsarten wird die Neunerprobe genannt. Alles wird in Worten erklärt und die benutzten Beispiele werden - wenigstens in dem überlieferten lateinischen. Text - nicht durch mit Ziffern geschriebene Zahlen erläutert, sondern die Zahlen werden in Worten dargestellt oder mit römischen Zahlzeichen. Es fehlt die Erklärung von dem, was man bei der Subtraktion zu thun habe, wemn eine Ziffer des Subtrahendus grösser ist als die entsprechende des Minuendus. Nach allem diesem wird es begreiflich, dass die indische Rechenkunst durch die Übersetzung dieses Werkes nicht sofort in Europa recht zugänglich werden konnte. Seine Entstehung bei den Arabern ist jedoch ein Zeugnis dafür, dass die indische Rechenkunst nunmehr bekannt 
war. Sie konnte sich dann mit Hülfe dieses Buches verbreiten, wenn es gehörig erklärt wurde; der Verfasser bezeichnet diese Art zu rechnen ausdrücklich als indisch.

Ebensowenig ist der Verfasser Schuld daran, dass man ihm später die Begründung der Algebra hat zuschreiben wollen. Er berichtet, dass Almamûn ihn aufgefordert habe ein kurzes Werk über Aldschebr und Almukâbala zu schreiben, das sich auf das Leichteste und Gebräuch. lichste in der Arithmetik und ihren praktischen Anwendungen beschränke. Die beiden Worte, von denen er es sogar überflüssig findet eine Erklärung zu geben, müssen also etwas bedeuten, das im voraus in weiterem Umfange bekannt war. Nach der sprachlichen Bedeutung der Worte und dem Zusammenhange, in dem sie gebraucht werden, bedeutet das erste die Operation, Glieder, die von der einen von zwei gleichen Grössen abgezogen werden sollen, $\mathrm{zu}$ der anderen hinzuzulegen, so dass die beiden gleichen Grössen jede nur positive Glieder enthalten. Das andere bedeutet die Operation, Glieder derselben Art, nämlich einfache Zahlen, Unbekannte und ihre Quadrate, zusammenzuziehen. Der Name für die erste von diesen Operationen, mit der die Behandlung der Gleichungen beginnen sollte, ist also auf die gesammte Lehre von den Gleichungen übertragen worden. Diese Lehre - und späterhin namentlich ihre Behandlung durch eine Zeichensprache, ja im allgemeinen die Lehre, von Operationen mit Grössen mittels .einer Zeichensprache - hat also einen Namen bekommen, der eigentlich einer einzelnen algebraischen Operation angehörte, die nicht einmal mehr auf diese Weise benutzt wird. Nun legen wir nämlich keinen Wert mehr darauf lauter positive Glieder auf jeder Seite des Gleichheitszeichens $\mathrm{zu}$ erhalten, so wie die Griechen, Araber und ihre nächsten europäischen Nachfolger es thaten. Wie alles, was von den griechischen Mathematikern her- 
rührt, hatte diese Operation indessen einen vernünftigen Grund. Man wollte sich nämlich durch diese Anordnung versichern, dass die Grössen, die man gleichsetzte, positiv waren, welchen (positiven) Wert die Unbekannte auch erhalten würde, denn man erkannte nur positive Grössen an.

Was wir in dem erwähnten Werke finden, ist namentlich die Behandlung von Gleichungen zweiten Grades und die Anwendung von diesen und von Gleichungen ersten Grades. Alles wird in Worten dargestellt, indem die Unbekannte während der Behandlung "Wurzel» oder «Ding» genannt wird, ihr Quadrat einfach «Quadrat». Die Lösung von Gleichungen zweiten Grades wird durch geometrische Algebra gezeigt, doch zum Teil durch andere Figuren wie bei Euklid. So wird die Gleichung

$$
x^{2}+a x=b
$$

durch folgende Figur gelöst. An die vier Seiten des unbekannten Quadrates $x^{2}$ werden Rechtecke mit den Höhen $\frac{a}{4}$ angelegt. Wenn die einspringenden Ecken der dadurch gebildeten Figur durch Quadrate mit der Seite $\frac{a}{4}$ aus. gefüllt werden, so soll das dadurch entstandene Quadrat mit der Seite $x+\frac{a}{2}$ den bekannten Wert $b+\frac{a^{2}}{4}$ haben.

Diese Form der Lösung, die auch bei anderen arabischen Schriftstellern vorkommt, und die wenigstens eine von Euklid unabhängige Anwendung der geometrischen Algebra darstellt, kann vielleicht aus uns unbekannten griechischen Arbeiten herrühren, z. B. aus Hipparchs bereits erwähnter Schrift über Gleichungen. Dass das Werk jedoch keineswegs in allen Stücken eine Bearbeitung eines griechischen Musters darstellt, sieht man aus den Anwendungen der Gleichungen auf praktische Ver- 
hältnisse des Lebens, z. B. auf das den Arabern eigentümliche Erbrecht. Gleichfalls muss angeführt werden, dass Muhammed ibn Mûsâ ebenso wie die Inder der Gleichung $x^{2}+a^{2}=b x$ zwei Wurzeln beilegt. Ausser dem griechischen Werte $\pi=\frac{22}{7}$ und dem möglicherweise griechischen Werte $\pi=3,1416$ kennt er den indischen Wert $\sqrt{10}$, ein Zeugnis dafür, dass er nicht das Zahlenrechnen allein von den Indern gelernt hat.

Was die Auflösung der Gleichungen zweiten Grades betrifft, so sieht man, dass Muhammed ibn Mûsâ nur dasselbe bringt, was sich bei griechischen Schriftstellern findet. Dass spätere Geschlechter dieses bei ihm, nicht aber immer bei den griechischen Schriftstellern, als sie von neuem bekannt wurden, haben finden können, beruht darauf, dass er Zahlenbeispiele $\mathrm{zu}$ den allgemeinen, in geometrischer Form dargestellten Lösungen hinzufügt, während Euklid sich damit begnügt diese zu geben, Hero nur einige numerische Anwendungen giebt, und Diophant die Lösung, die er aufstellt, nicht beweist.

Machen wir nun einen Sprung bis zu der Zeit um das Jahr 1000, so treffen wir auf zwei sehr verschiedene Behandlungen der Arithmetik und des Rechnens. Aus der einen, die von Alnasawî herrührt, sehen wir, dass damals grosse Fortschritte in der Benutzung der indischen Rechenmethode gemacht waren und damit im ganzen in geregelter Darstellung und Behandlung von Zahlengrössen. So giebt es Bezeichnungen für Brüche, die mit unseren Ziffern - denn die Zahlzeichen sind nicht überall dieselben, wo das Positionssystem benutzt wird - aussehen würden wie in den folgenden Beispielen:

$$
\frac{1}{11}=\begin{gathered}
0 \\
11
\end{gathered} ; 15_{\frac{7}{19}}=\begin{array}{r}
15 \\
7 \\
19
\end{array}
$$


Da sich aus dem hier erwähnten Buche ergiebt, wie gut die indische Rechenmethode eingedrungen und verstanden war, so ist es überraschend, zu derselben Zeit und an demselben Orte ein Rechenbuch von dem bedeutenden Mathematiker Alkarchî entstehen zu sehen, in dem sich gar nichts von der indischen Ausführung der Zahlenrechnungen findet. Dagegen werden die Zahlen in Worten mitgeteilt, und selbst weitläufige Rechnungen werden ohne Benutzung der Ziffern ausgeführt. Das scheint auf einen principiellen Widerstand gegen die indische Rechenmethode hinzudeuten, und man hat die recht wohl annehmbare Vermutung aufgestellt, dass dieser von den scharfen Gegensätzen zwischen religiösen Sekten herrühren könne. Neben solchen Erklärungen darf man jedoch nicht unterlassen zu prüfen, ob der Unterschied zwischen Alnasawî und Alkarchî nicht auch in dem verschiedenen $Z i e l$, das sie sich stecken, liegen kann. Der erste hat eben die Regeln für die einfachste praktische Ausführung von Zahlenrechnungen geben wollen; der zweite dagegen hat ein wissenschaftliches Werk über Zahlen und die Benutzung von Zahlen schreiben wollen, und da hat er mit gutem Grunde seinen Ausgangspunkt bei den Griechen und nicht bei den Indern gesucht. Wenn er dennoch die Regula de tri der Inder mitnimmt, so giebt er ihr eine sichere Grundlage in Euklids Proportionslehre. Wenn er es unterlässt solche mechanische Hülfsmittel mitzuteilen, die wirklich dazu dienen können die vorliegenden Zahlenrechnungen $\mathrm{zu}$ bewältigen, so geht er nicht einmal so weit wie Euklid, der nicht nur ganz von den mechanischen Hülfsmitteln schweigt, die man auch zu seiner Zeit gehabt haben muss, sondern auch nicht ein einziges Zahlenbeispiel giebt. Dass Alkarchî dennoch Veranlassung findet eine Anzahl griechischer Methoden des Rechnens zu erklären, die weit hinter den 
indischen zurückstehen, kann von seiner Bewunderung für die Griechen herrühren. Diese kann ihm ein theoretisches Interesse für den Zusammenhang dieser Rechenmethoden verliehen haben, das man für die indischen Rechenmethoden noch nicht besass.

Wie nun auch der Unterschied zwischen den beiden Schriftstellern zu erklären sein mag, immerhin zeigt er, dass die Verschmelzung der griechischen und indischen Beiträge zu Mathematik und Rechnen Zeit erforderte. Beide Bücher zeigen jedoch zugleich, dass man jetzt beide in recht bedeutendem Umfange $\mathrm{zu}$ seiner Verfügung hatte.

Dass auch Alkarchî jedenfalls verstand mit Zahlen umzugehen, und zwar unter Benutzung anderer mechanischer Hülfsmittel als in seinem Rechenbuche zu finden sind, das geht teils aus den weitläufigen Rechnungen hervor, die sich immer noch in diesem Buche finden, teils aus seinem bedeutenderen algebraischen Werke Alfachrî, wie es wahrscheinlich nach einer Person genannt ist. In diesem zeigt er sich als ein hervorragender Schüler von Diophant, der nicht nur in grossem Umfange dessen Untersuchungen und Beispiele wiedergiebt, sondern zugleich selbst bedeutende Fortschritte macht. In dieser Beziehung lässt sich anführen, dass er Diophants Zeichensprache erweitert, ja sogar an einzelnen Stellen Zeichen für zwei Unbekannte benutzt; dass er vollständigere Regeln für das Rechnen mit Grössen giebt, in denen eine Unbekannte vorkommt, und dass er viele andere Beispiele behandelt als diejenigen, die sich bei Diophant finden, ja dass er sich sogar auf neue Arten von unbestimmten Aufgaben einlässt. Als Beispiel hierfür mögen die Gleichungen

$$
y^{2}=x^{3}+a x^{2}, z^{2}=x^{3}+b x^{2}
$$

angeführt werden; setzt man $y=m x, z=n x$, so folgt 


$$
x=m^{2}-a=n^{2}-b,
$$

wo $m^{2}$ und $n^{2}$ willkürlich gewählte Quadratzahlen mit der Differenz $a-b$ sind.

Grösseres Gewicht jedoch wollen wir auf die mehr begriffsmässigen Fortschritte legen, die wir antreffen. Damit diese verstanden werden können müssen wir bemerken, dass Alkarchî sich nicht nur Diophants praktische Behandlungsmethoden angeeignet hatte, sondern dass er auch, wie er ja bereits in seiner Arithmetik gezeigt hatte, ein vollkommenes Verständnis von dem hatte, was nach griechischer Denkweise für einen Beweis erforderlich.war. Geometrische Beweise, die ja die einzige Form für allgemeingültige Beweise waren, giebt er jedoch nur für Lösungen von Gleichungen zweiten Grades, und selbst da bewegt er sich mit grösserer Freiheit als die Griechen, da er in einem von diesen Beweisen $x^{2}$ und $a x$ durch Strecken darstellt, etwas, was ein griechischer Schriftsteller in einem Beweise nur indirekte dadurch würde machen können, dass er $x^{2}$ und $a x$ in Rechtecke mit derselben Seite verwandelte. Er begnügt sich indessen damit die meisten Regeln durch ein einziges Beispiel zu erläutern, das zeigen soll, wie sie aus den Rechnungen selbst hervorgehen. Ja er bemerkt sogar ausdrücklich, dass man sich auf das Verständnis der Regeln für algebraisches Rechnen vorbereiten müsse durch die allgemeinen arithmetischen Regeln, die er in seinem früherem Werke gegeben habe. Er verspricht derartige arithmetisch-algebraische Regeln in grösserem Umfange in einem Werke $\mathrm{zu}$ geben, das wir nicht besitzen.

An und für sich liegt vielleicht nichts Neues in diesen Betrachtungen, denn das wirkliche Rechnen mit rationalen Zahlen musste auch für die Griechen in vielen Beziehungen das Vorbild für die geometrische Behandlung gewesen sein, durch die sie es dahin brachten, dass dieselben 
Operationen irrationale Grössen umfassten. Von grosser Bedeutung aber ist es, dass diese Betrachtungen ausdrücklich hervorgehoben werden. Dies zeigt sich bei Alkarchî in der Freiheit, mit der er irrationale Wurzelgrössen behandelt. Diese werden allerdings nicht durch Zeichen dargestellt, sondern in Worten, die den Benennungen von Potenzen mit demselben Exponenten entsprechen; aber wie bei den Indern wird ausdrücklich gezeigt, wie man mit ihnen rechnen kann, nämlich teils wie sie multipliciert und dividiert werden können, welchen Wert die Potenz auch haben mag, teils wie Quadrat- und Kubikwurzeln addiert und subtrahiert werden können, wenn die Potenzen ähnliche ebene oder räumliche Zahlen sind. Die letzten Sätze werden nicht dadurch bewiesen, dass rationale Faktoren mittels der ersten Sätze ausserhalb des Wurzelzeichens gebracht werden, sondern durch Anwendung der Formeln für $(a \pm b)^{2}$ und $(a \pm b)^{3}$.

Wir sehen also, dass Alkarchî mit irrationalen Wurzelgrössen rechnet, oder mit anderen Worten, dass er sie auch als Zahlen auffasst. Indirekt thut er das auch dadurch, dass mehrere von seinen bestimmten Gleichungen zu irrationalen Wurzeln führen, dass also in den Gleichungen, die zu diesen geführt haben, die Zeichen, die unserem $x^{m}$ entsprechen, Potenzen von irrationalen Zahlen darstellen, während Diophant stets voraussetzt, dass $x$ eine rationale Zahl sein soll. Nun haben wir allerdings gesehen, dass die Inder ohne Skrupel mit irrationalen Zahlen rechneten, aber diesen ist Alkarchî wissentlich kaum gefolgt. Von Bedeutung bleibt es, dass wir hier dasselbe von einem Manne thun sehen, der von den griechischen Schriftstellern her vollkommen mit dem Begriffe des Irrationalen vertraut ist, und der durch die Weise, wie er zwischen den geometrischen Beweisen und den arithmetischen Erklärungen unterscheidet, zu erkennen 
giebt, dass er sich bewusst ist, dass die letzteren keine allgemeingültigen Begründungen liefern. Als Schüler der Griechen konnten die Araber deshalb nicht bei den arithmetischen Erklärungen stehen bleiben. Das sehen wir aus der Algebra, die uns von dem angesehenen arabischen Mathematiker 'Omar Alchaijâmî, der im 11ten Jahrhundert lebte, hinterlassen ist. Dieser setzt seine Erklärungen von der Bedeutung der irrationalen Wurzelgrössen in direkte Verbindung mit den strengen griechischen Begriffen. Er unterscheidet zwischen arithmetischen und geometrischen Auflösungen von Gleichungen. Die ersten will er nicht nur - wie Diophant, und was in diesem Zusammenhange ausreichend sein würde - rational haben, sondern auch ganz. Da sich mit diesen Grössen rechnen lässt, so ist ein arithmetischer Beweis für die Richtigkeit solcher Auflösungen ausreichend. Die Auflösungen der zweiten Art können irrational sein, und deshalb müssen sie geometrisch dargestellt werden und verlangen einen geometrischen Beweis. Quadratwurzeln und Kubikwurzeln werden dann durch die von den Griechen her bekannten Konstruktionen von einer und zwei mittleren Proportionalen dargestellt. Für Wurzelgrössen höherer Ordnung lässt sich, weil der Raum nur drei Dimensionen hat, keine geometrische Darstellung durchführen, und eine andere allgemeine Darstellung kennt Alchaijâmi nicht. Bei der Bildung von Potenzen höherer Ordnung weist er dagegen in Übereinstimmung mit Euklids Proportionslehre auf die Bildung zusammengesetzter Verhältnisse hin, wodurch auch indirekt eine Erklärung von dem gegeben wird, was die irrationalen Wurzelgrössen höherer Orddnung, die wir bei Alkarchî getroffen haben, bedeuten müssen. Alchaijâmis Auffassung, deren theoretische Bedeutung Alkarchî vermutlich auch anerkannt haben würde, ist also vollkommen griechisch. 
Was die Berechnung von Wurzelgrössen betrifft, so verweist Alchaijâmi auf das Ausziehen der Quadrat- und Kubikwurzel bei den Indern, und fügt hinzu, dass er selbst anderswo - dann aber in einem. unbekannten Werk - Regeln für das Ausziehen von Wurzeln mit einem beliebigen Exponenten gegeben habe.' Dazu muss er sicherlich Binomialkoefficienten für ganze Exponenten gekannt haben, muss also mit den Regeln für die Bildung dieser Koefficienten bekannt gewesen sein. Er sagt, dass er das Ausziehen dieser Wurzeln nur arithmetisch gezeigt habe. Dann hatte es für ihn auch nur Gültigkeit, wenn es sich mit Genauigkeit ausführen liess. Ohne dies hat er ja nach dem Vorhergehenden dessen wirkliche Bedeutung sogar nur angedeutet. Indessen ist es klar, dass sowohl das Ausziehen solcher Wurzeln wie dasjenige von Quadrat- und Kubikwurzeln sich auch für eine genäherte Berechnung irrationaler Wurzeln benutzen lässt. Als Beispiel für die Beschäftigung mit genähertem Wurzelausziehen können wir im übrigen anführen, dass Alkarchî für $\sqrt{a^{2}+r}$, wenn $a$ die nächst kleinere ganze Zahl ist, $a+\frac{r}{2 a+1}$ als weiteren Näherungswert angiebt. Diesen Wert erhält man, wenn man die Regel der zwei falschen Ansätze (S. 275) oder die Interpolation zwischen $a$ und $a+1$ anwendet.

Wie verschieden die hier erwähnte Beschäftigung mit Wurzelgrössen bei Alkarchî und Alchaijâmî auch sein mag, so musste sie doch dazu beitragen bei den Arabern ein Problem hervorzuziehen, welches durch die griechische Behandlungsweise etwas verdeckt worden war, nämlich die Lösung der kubischen Gleichung durch Quadrat- und Kubikwurzeln. Wenn die Griechen sich auch möglicherweise in älteren Zeiten mit dieser Aufgabe beschäftigt haben, so musste sie doch etwas von ihrem Interesse da- 
durch verlieren, dass man die kubischen Gleichungen direkt durch eben dieselben geometrischen Mittel lösen konnte, die gleichfalls zu einer allgemeingültigen Darstellung der Kubikwurzel dienen mussten, nämlich das Durchschneiden von Kegelschnitten. Ja wie wir gesehen haben, verschwand das Interesse dafür, so wie Archimedes es gethan, Aufgaben auf kubische Gleichungen zurückzuführen, da man sah, dass man auch ohne eine solche Zurückführung Aufgaben durch eben dieselben Hülfsmittel lösen konnte.

Es gelang den Arabern nun allerdings nicht die Lösung der kubischen Gleichung zu finden, aber das Interesse, das sie ihr bewiesen, tritt in zahlreichen Untersuchungen hervor. Der wichtigste Ausgangspunkt für diese Untersuchung war teils Archimedes' Aufgabe von der Kugelteilung, teils die alte Auflösung dieser Aufgabe durch Kegelschnitte (vergl. S. 217 ff.), von der man annimmt, dass sie von Archimedes oder doch aus seiner Zeit stammt. Da diese, wie wir gesehen haben, alle Gleichungen von der Form

$$
x^{3}+a x^{2}+b=0
$$

umfasst oder doch leicht dahin gebracht werden kann sie zu umfassen, da ferner die Bedingung für gleiche Wurzeln ausdrücklich angegeben wird, und da man leicht entweder die allgemeine Gleichung dritten Grades auf diese Form zurückführen oder sie wesentlich auf dieselbe Weise behandeln kann, so waren auf diesem Punkte besonders grosse wissenschaftliche Schwierigkeiten nicht zu überwinden. Die Araber führten indessen die Untersuchung der kubischen Gleichungen insofern weiter, als sie Einteilungen der Gleichungen dritten Grades aufstellten, teils nach den Vorzeichen der Koefficienten, teils nach den Werten von diesen, die zu mehr oder weniger Wurzeln 
führen. Die vollständigste von diesen Einteilungen findet sich in ${ }^{\circ} \mathrm{Omar}$ Alchaijâmîs Algebra. Für den einzelnen Fall wird gezeigt, wie die Aufgabe sich durch Kegelschnitte lösen lässt und wie viele Wurzeln sie erhält, d. h. positive Wurzeln, denn nach anderen fragen die Araber nicht. Alchaijâmîs Einteilung leidet jedoch an Mängeln, die davon herrühren, dass er nicht den Diorismus genau angiebt, der bei der griechischen Behandlung die Hauptausbeute aus der Lösung durch Kegelschnitte ist. Anderen arabischen Schriftstellern, namentlich Alkûhî, gelingt dies besser im Anschluss an das durch Eutokius überlieferte Manuskript.

Dadurch dass die Gleichungen dritten Grades bestimmter hervorgezogen wurden als es in der jetzt und damals überlieferten griechischen Geometrie der Fall war, wurden sie auch ein mehr bestimmtes Durchgangsglied für die Lösung von anderen Aufgaben, sowohl von solchen, die von den Griechen her vorlagen, als auch von neuen. Unter den ersteren fand namentlich die Dreiteilung des Winkels eine grosse Menge von Auflösungen. So haben wir diejenige, die man wegen ihres Zusammenhanges mit den archimedischen Hülfsätzen vielleicht dem Archimedes zuschreiben darf (S. 79), von den Arabern erhalten. Alkûhî fand auch die Lösung der Aufgabe, einen Kugelabschnitt aus seinem Volumen und seiner krummen Oberfläche zu bestimmen, und schloss daran eine Begründung des zu dieser Aufgabe gehörigen Diorismus, den Archimedes am Schlusse des 2ten Buches über die Kugel und den Cylinder mitteilt (vergl. S. 185 und 220).

$\mathrm{Da}$ es den Arabern nicht gelang, eine allgemeine Lösung der Gleichungen dritten Grades durch Wurzelgrössen zu finden, so mussten sie, wenn praktische Berechnungsaufgaben auf Gleichungen dritten Grades führten, sich an eben diese halten - was im übrigen ja auch 
jetzt noch leichtere Mittel für die Berechnung liefert als die Anwendung der allgemeinen Lösung -. Ein sehr hübsches Beispiel für eine numerische Berechnung einer Wurzel einer kubischen Gleichung ist uns erhalten geblieben. Sie rührt wahrscheinlich von dem Arzte Alkâschî aus dem 15ten Jahrhundert her, und geht darauf aus $\sin 1^{0} \mathrm{zu}$ finden, der, da $\sin 3^{0}$ bekannt ist, von einer Gleichung von der Form

$$
x^{3}+Q=P x
$$

abhängt. Da $x$ klein ist, so kann man es mit einer gewissen Annäherung gleich $\frac{Q}{P}$ setzen. Für diese Grösse wird ein solcher Näherungswert $a$ berechnet, dass der Divisionsrest $R$ klein von derselben Ordnung wie $a^{3}$ wird; dann wird $x=a+y$ gesetzt oder

woraus

$$
\begin{gathered}
a+y=\frac{(a+y)^{3}+Q}{P} \\
y=\frac{(a+y)^{3}+R}{P} .
\end{gathered}
$$

Da der Rest $R$, der von derselben Ordnung ist wie $a^{3}$, im Vergleich zu $a^{2} y$ gross ist, so kann man in einer angenäherten Berechnung die Glieder im Zähler, die y enthalten, fortlassen, und man erhält dann mit einer neuen Annäherung

$$
y=\frac{a^{3}+R}{P}=b+\frac{S}{P}
$$

Dann wird in die genaue Gleichung $y=b+z$ eingesetzt, worauf wieder $z$ auf ähnliche Weise mit Annäherung bestimmt wird. - Die Brüche werden als Sexagesimalbrüche dargestellt. 
Das Ziel der hier angeführten Berechnung gehört unter die Trigonometrie, mit der wir uns bald beschäftigen werden; aber bevor wir die Arithmetik, Algebra und Zahlentheorie der Araber verlassen, bei denen wir bis dahin namentlich die verschiedenen allgemeinen Auffassungen berücksichtigt haben, wollen wir einige Proben von den Resultaten mitteilen, die innerhalb dieser Gebiete, namentlich in der Zahlentheorie gewonnen sind. Im 9ten Jahrhundert hat Thâbit ibn Kurra, im Anschluss an Euklids Bestimmung von «vollkommenen Zahlen» (S. 158), Regeln gegeben für die Bestimmung solcher Zahlen, die die Pythagoreer "befreundete Zahlen» nannten, d. h. solche, von denen die eine gleich der Summe der Faktoren der anderen ist. Die Regel lautet folgendermassen: Sind $p=3.2^{n}-1, q=3.2^{n-1}-1, r=9.2^{2 n-1}-1$ lauter Primzahlen, so sind $2^{n} \cdot p \cdot q$ und $2^{n} \cdot r$ befreundete Zahlen.

Vom 10ten Jahrhundert an haben die Araber sich mit den sogenannten Zauberquadraten beschäftigt; bei diesen werden die dazu sich eignenden Zahlen so in Quadraten zusammengestellt, dass die Summen der Zeilen, Kolumnen und Diagonalen gleich gross sind. Das älteste Beispiel für ein solches Zauberquadrat findet sich in einer vielleicht 4-5000 Jahre alten chinesischen Tafel; es ist

$\begin{array}{lll}8 & 3 & 4 \\ 1 & 5 & 9 \\ 6 & 7 & 2 .\end{array}$

Die Araber bildeten auch Zauberquadrate aus den Zahlen bis 16,25 und 36 , und gaben an, dass ähnliche sich aus den Zahlen bis 49, 64 und 81 bilden liessen. Übrigens haben indische und byzantinische Mathematiker sich auch mit demselben Gegenstand beschäftigt. 
Von grösserem mathematischen Interesse ist ein zahlentheoretischer Satz, den Alchodschandî ungefähr um das Jahr 1000 fand, dass nämlich die Gleichung $x^{3}+y^{3}=z^{3}$ sich nicht rational lösen lässt.

Bei Alkarchî trifft man die von den Griechen her bekannten Summationen von $1^{2}+2^{2}+3^{2}+\ldots$ und $1^{3}+2^{3}+3^{2}+\ldots$ Mit dem Beweise für die erste kann er jedoch nicht fertig werden; er kennt also nicht den Beweis von Archimedes. Für die zweite giebt er dagegen den Beweis, den wir anführten, als wir von der Bekanntschaft der Griechen mit diesem Satze sprachen (S. 245). Dagegen bedeutet es einen wirklichen Fortschritt, wenn der bereits genannte Alkâschî die Reihe $1^{4}+2^{4}+3^{4}+\ldots+r^{4}$ summiert; als Summe giebt er an

$$
\begin{gathered}
\left(\frac{(1+2+\ldots+r)-1}{5}+(1+2+\ldots+r)\right) \\
\left(\left(1^{2}+2^{2}+\ldots+r^{2}\right) .\right.
\end{gathered}
$$

\section{Die Trigonometrie der Araber.}

Ein Gebiet, auf dem man den Arabern bei ihrem Vertrautsein mit griechischer Geometrie und indischer Rechenkunst umfassendere Fortschritte zu verdanken hat, ist die berechnende Geometrie oder die Trigonometrie, wie wir sie jetzt um so mehr nennen können, als die Araber wie die Inder Sinustafeln statt der Sehnentafeln des Ptolemäus benutzten. Da der Name sinus auch insofern indischen Ursprung hat, als er die richtige lateinische Übersetzung eines arabischen Wortes ist, das durch Entstellung des indischen Wortes für sinus entstanden war, so können die Inder allerdings Veranlassung zu dieser Änderung gegeben haben, aber die Araber haben 
ihre Trigonometrie der Hauptsache nach nicht von ihnen gelernt. Die Änderung ist nämlich ausdrücklich von dem Astronomen Albattânî eingeführt als eine Erleichterung der vom Almagest her bekannten Berechnungsmethoden.

Bei der Berechnung der Tafel kam es nun namentlich darauf an $\sin 1^{0}$ oder $\sin \frac{1}{2}^{\circ} \mathrm{zu}$ finden, die sich ja nicht durch quadratische Gleichungen bestimmen lassen. Wir haben soeben eine von einem späteren Schriftsteller benutzte numerische Lösung der dazu dienenden kubischen Gleichung kennen gelernt. Früher benutzte man sowohl hier wie später bei der Berechnung von $\sin 10^{\prime}$ eine Interpolation zwischen solchen sinus, die sich durch Quadratwurzeln ausdrücken liessen, und die im wesentlichen von derselben Art war wie diejenige, wodurch Ptolemäus die Sehne von $1^{0}$ fand. Eine solche wendet z. B. der grosse Astronom und Mathematiker A bûl Wafâ in der zweiten Hälfte des 10ten Jahrhunderte an. Die Araber blieben indessen nicht bei Sinustafeln stehen, sondern Abûl Wafâ berechnete zugleich Tangententafeln. Was die Genauigkeit der Tafeln betrifft, so gelangte man $\mathrm{zu}$ Sinustafeln von $10 \mathrm{zu} 10$ Minuten mit der Fehlergrenze $\frac{1}{60^{4}}$. A hûl Wafâs Tangententafeln haben sogar die Fehlergrenze $\frac{1}{60^{5}}$.

Was nun die Anwendung der Tafeln betrifft, so finden wir bereits bei Albattânî die Formel

$$
\cos a=\cos b \cos c+\sin b \sin c \cos A \text {. }
$$

Im übrigen hielt man sich der Hauptsache nach an Ptolemäus' Formeln (vergl. S. 234), die für die Benutzung von Sinustafeln umgeformt worden waren. Gewöhnlich geschieht dies ohne Aufstellung eines Beweises. Beweise, die zugleich von Ptolemäus' Anwendungen des sogenannten Theoremes von Menelaus abweichen 
finden wir bei Dschâbir ibn Aflah, bekannter unter dem Namen Geber, der im 11ten Jahrhundert in Sevilla in Spanien lebte. $\mathrm{Ihm}$ gebührt $\mathrm{zu}$ gleicher Zeit die Ehre, die zu dem rechtwinkeligen sphärischen Dreieck gehörenden Formeln durch eine Relation zwischen zwei Winkeln und einer Seite ergänzt zu haben. Diese wird durch dieselbe Figur gefunden, die Ptolemäus benutzte, in der $D E F$ ein grösster Kreis ist, der zum Pol den Scheitelpunkt des Winkels $A$ in dem bei $B$ rechtwinkeligen Dreieck $A B C$ hat. Das gleichfalls rechtwinkelige Dreieck $D E C$ hat

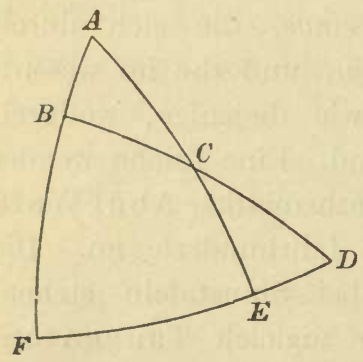
nämlich den Winkel $C$ mit $A B C$ gemeinsam, und $D E=$ $90^{\circ}-A, C D=90^{\circ}-a$ woraus folgt, dass $\cos A=\cos a \sin C$. Dieser Satz heisst derGebersche. Geber ist der einzige Westaraber, von dem zu sprechen wir Veranlassung haben. Yon der westarabischen Mathematik, die sich am unmittelbarsten zu den europäischen Völkersahaften verbreitete, wollen wir im übrigen nur bemerken, dass die arithmetisch-algebraische Behandlung sich bei ihr mehr als bei den ostarabischen Schriftstellern von den von den Griechen herrührenden geometrischen Formen befreite. Hiermit waren einige Erweiterungen in der Benutzung mathematischer Zeichen verbunden, beispielsweise die Einführung eines Zeichens für die Quadratwurzel. Die Ehrfurcht vor den griechischen Schriftstellern dauerte jedoch bei den Westarabern fort, so dass man durch diese in Europa die griechischen Schriftsteller kennen lernte, wenn auch bedeutend langsamer, als es geschehen wäre, wenn man die Ostaraber zu Lehrern gehabt hätte. Dafür kam Rechnen, Arithmetik und Algebra in leichter 
zugänglichen Formen nach Europa. Die höchste Vorstellung von der Matkematik der Westaraber erhält man durch ihren ersten grossen europäischen Schüler L,eonardo von Pisa: denn ein wie grosses mathematisches Genie er auch selbst sein mochte, der Umfang seiner Kenntnisse zeugt dennoch auch sowohl von der Tüchtigkeit seiner Lehrer, als auch von ihrem umfassenden Wissen und ihrer genauen Bekanntschaft mit der älteren arabischen und dadurch auch mit der griechischen Mathematik.

\section{Erstes Wiedererwachen der Mathematik in Europa.}

Es kann nicht unsere Absicht sein uns hier mit Einzelheiten in der bescheidenen Entwickelung zu beschäftigen, die das von den Römern empfangene Rechnen auf dem Abacus in den Klöstern erfuhr, oder mit den Wegen, auf welchen andere Mittel des Rechnens und eine bessere Mathematik als die von den Römern überlieferte von den Arabern her in Europa eindrang. Wenn wir jedoch nun den grössten europäischen Mathematiker des Mittelalters, Leonardo von Pisa, etwa 1200, besprechen wollen, so müssen wir, um ihm den rechten Hintergrund $\mathrm{zu}$ geben, berühren, wie weit man $\mathrm{zu}$ seiner Zeit sonst in Europa gekommen war. Damals lagen Übersetzungen aus dem Arabischen vor von Rechenbüchern ("Algorithmen»), von einer Algebra mit Gleichungen vom ersten und zweiten Grade, ja von Euklids Elementen und Ptolemäus' Almagest; aber die einzelnen handschriftlichen Exemplare waren rein äusserlich nur äusserst wenigen zugänglich, und das wirkliche Eindringen in den Inhalt und das Vermögen ihn zu benutzen war bei 
diesen gewiss auch sehr begrenzt. Das algorithmische, d. h. indische Rechnen war bereits damals im Begriff etwas Verbreitung in gelehrten Kreisen an mehreren Orten zu gewinnen, während andere das Rechnen auf dem Abacus benutzten, zu dessen Förderung einige Jahrhunderte früher Gerbe $\mathbf{r} t$, der spätere Papst Sylvester II beigetragen hatte, und bei dem man nun wohl meistens die Zahlzeichen auf die eingeteilten Kolumnen schrieb (S. 293). Der Unterschied zwischen den beiden Rechenmethoden bestand also darin, dass die algorithmische durch Benutzung des Zeichens 0 die Einteilung in Kolumnen entbehren konnte. An die verschiedenen Rechenmethoden knüpften sich übrigens verschiedene Traditionen, darunter die für die Algorithmiker etwas herabsetzende, dass sie dauernd dem Muhammed ibn Mûsâ darin folgten, Verdoppelung und Halbierung als besondere Rechnungsarten auszuscheiden. Dagegen hatten sie den Vorzug, dass sie nicht nur wie die Abacisten Quadratwurzeln, sondern auch Kubikwurzeln kannten. Die Algorithmiker benutzten Sexagesimalbrüche, während die Abacisten zum Teil fortfuhren die vom Müntzsystem der Römer stammende Zwölfteilung zu gebrauchen.

Leonardo Fibonacci, das heisst Sohn des Bonaccio, wie man ihn oft nach dem Beinamen seines Vaters nennt, stammt aus der wichtigen Handelsstadt Pisa, wo er frühzeitig das Rechnen auf dem Abacus lernte. Auf Geschäfts- (oder vielleicht Dienst-) reisen besuchte er dann Ägypten, Syrien, Griechenland, Sicilien und die Provence, wobei er sich weiter in Rechenkunst und Mathematik ausbildete. Was er auf diese Weise lernte, das suchte er dem "lateinischen Volksstamme» durch sein umfangsreiche Werk Liber abaci zugänglich zu machen, in dem er mit überlegener Tüchtigkeit zahlreiche Beispiele so $\mathrm{zu}$ sagen für alles Rechnen giebt und 
4. Erstes Wiedererwachen der Mathematik in Europa. 317

behandelt, auf das wir bis dahin getroffen sind: Rechnen mit ganzen Zahlen, die nach dem Positionssystem geschrieben sind, und mit Brüchen; alle Arten kaufmännisches Rechnen; Lösung von Aufgaben, die auf eine Gleichung gebracht, von Gleichungen ersten Grades abhängen würden, mit Hülfe der beiden Regeln des falschen Ansatzes und der indischen Umkehrungsmethode; Differenzreihen erster und zweiter Ordnung; Aufgaben, die von Quotientenreihen abhängen, oder die, wie diejenige über die Vermehrung der Kaninchen, als Aufgaben über Zinseszinsrechnung gelöst werden; zum Teil solche, die von unbestimmten Gleichungen ersten Grades abhängen - ohne dass Leonardo jedoch wie die Inder bestimmte Regeln für ihre Auflösung giebt -; Ausziehen der Quadrat- und Kubikwurzel; endlich Aufgaben, die von bestimmten und unbestimmten Gleichungen zweiten Grades abhängen.

Der Name Liber abaci scheint nicht ganz dazu zu stimmen, dass Leonardo durchweg das Zahlzeichen 0 gebraucht. Indessen weiss man, dass er selbst von vorn herein in der Benutzung des Abacus unterrichtet worden ist. Die indische Rechenkunst hat er dagegen direkt von den Arabern gelernt, und er ist ihr vielleicht kaum einmal in Europa begegnet, wo sie wohl nur in einzelnen geistlichen Kreisen bekannt gewesen ist. Dass er nicht ursprünglich Algorithmiker ist, ergiebt sich daraus, dass er, wie er sagt, selbst das Ausziehen der Kubikwurzel gefunden hat. Dies findet sich noch nicht bei Alkarchî, der von den bekannten arabischen Schriftstellern derjenige ist, von dem er am stärksten beeinflusst $z u$ sein scheint. Von ihm hat er eine grosse Menge Aufgaben entlehnt, die er jedoch mit Selbstständigkeit behandet. Es stimmt auch zu Alkarchîs angenäherter Berechuung einer Quadratwurzel (S. 307), wenn Leonardo eine weitere Annäherung an eine Kubikwurzel, von der die Anzahl 
der Ganzen bereits gefunden ist, durch die Regel der zwei falschen Ansätze bestimmt. Ist $\sqrt[3]{a+r}$ die gesuchte Kubikwurzel und sind $a$ die darin enthaltenen Ganzen,

so wird der Näherungswert $a+\frac{r}{3 a^{3}+3 a+1}$.

Leonardos Darstellung ist im Liber abaci durchweg von wirklichen Beweisen in geometrischer Form begleitet. Dasselbe ist der Fall mit seiner Practica geometrica, die unter anderem auch Anzüge aus den damals äusserst wenig bekannten stereometrischen Büchern Euklids enthält. Seine Beweise sind oft verschieden von denjenigen Euklids. Dadurch dass er überhaupt Beweise giebt, weicht er nicht nur von den geometrischen Arbeiten ab, die damals in Europa entstanden, sondern auch von vielen arabischen Schriftstellern.

In den hier genannten Schriften macht Leonardo in klarer Form, die selbstständige Aneignung und freie Behandlung verrät, die wichtigsten vorher bekannten arithmetischen, algebraischen und elementar geometrischen Sätze zugänglicher als sie durch Übersetzung werden würden, und erläutert namenlich die ersten durch zahlreiche Beispiele. Sein eigenes Vermögen bedeutende Schwierigkeiten $\mathrm{zu}$ überwinden tritt dagegen am besten hervor in seinen Lösungen von einigen Aufgaben, die ihm in Gegenwart Kaiser Friedrichs II vom Philosophen des Kaisers, Magister Johannes von Palermo, gestellt wurden. Die eine von diesen lief darauf hinaus eine Quadratzahl zu finden, die, um die Zahl 5 vermehrt oder vermindert, neue Quadratzahlen giebt, oder die Gleichungen

$$
\left.\begin{array}{l}
y^{2}=x^{2}+a \\
z^{2}=x^{2}-a
\end{array}\right\}
$$

rational zu lösen, wenn $a=5$. Die Gleichungen (1) waren früher von arabischen Mathematikern behandelt worden, 
und diese hatten gefunden, dass sowohl $x^{2}+a$ wie $x^{2}-a$ Quadratzahlen sind, wenn

$$
x=m^{2}+n^{2}, a=4 m n\left(m^{2}-n^{2}\right) .
$$

Diese Resultate ergeben sich übrigens leicht aus Diophants gewöhnlicher Behandlung von «doppelten Gleichungen» (S. 251; siehe auch die erste Aufgabe S. 254) in Verbindung mit Euklids Bestimmung von rationalen rechtwinkeligen Dreiecken. Leonardo gelangt auf einem etwas verschiedenen Wege zu demselben Resultat, indem er den Satz benutzt, dass die Quadratzahlen Summen der ersten ungeraden Zahlen sind.

Demnächst kommt es darauf an $m$ und $n$ so zu bestimmen, dass

$$
4 m n\left(m^{2}-n^{2}\right)
$$

einen gegebenen Wert erhält, im vorliegenden Falle 5. Leonardo beweist zuerst, dass Zahlen von dieser Form, wenn $m$ und $n$ ganze Zahlen bedeuten, durch 24 teilbar sind. Um womöglich Gleichungen $\mathrm{zu}$ erhalten, die sich in ganzen Zahlen lösen lassen, muss man also die gegebenen Gleichungen mit einem solchen Quadrat multiplicieren, dass das neue a durch 24 teilbar wird. Leonardo multipliciert mit $12^{2}$ und findet, dass

$$
5.12^{2}=4.5 .4(5+4)(5-4),
$$

wonach $41^{2} \pm 5.12^{2}$ Quadratzahlen werden. Die gesuchten Quadrate findet man dann durch Division mit $12^{2}$; es sind $\left(\frac{31}{12}\right)^{2},\left(\frac{41}{12}\right)^{2}$ und $\left(\frac{49}{12}\right)^{2}$.

In seiner sehr allgemein angelegten Untersuchung findet Leon ardo Gelegenheit eine allgemeine Bestimmung von der Summe der ersten ungeraden Quadratzahlen bis zu einer gewissen Grenze hinauf zu geben. Leonardos Lösung bringt also mehr als wonach er gefragt ist. 
Eine andere der gestellten Aufgaben verlangt $x$ aus der Gleichung

$$
x^{3}+2 x^{2}+10 x=20
$$

zu bestimmen. Leonardo findet zuerst, dass $x$ zwischen 1 und 2 liegt, also keine ganze Zahl sein kann. Darauf weist er nach, dass $x$ kein rationaler Bruch sein kann, und auch keine von den irrationalen Grössen, die Euklid in seinem 10ten Buche aufstellt. Den Inhalt dieses schwierigen Buches, das er studierte um darin eben möglicherweise Formen für exakte Ausdrücke für die Wurzeln der vorgelegten Gleichung $\mathrm{zu}$ finden, hat Leonardo vollkommen richtig verstanden, wenn er auch, wie er sagt, Zahlen an die Stelle der in geometrischer Form dargestellten allgemeinen Grössen setzt.

Da die Wurzel nun keine Grösse von bekannter Form ist, so muss Leonardo sich damit begnügen einen Näherungswert zu suchen. Diesen drückt er in Sexagesimalbrüchen aus und findet

$$
x=1^{0} 22^{\prime} 7^{\prime \prime} 42^{\prime \prime \prime} 33^{I V} 4^{V} 40^{V I},
$$

ein Resultat, das nur etwa um $1 \frac{1}{2}^{V I} \mathrm{zu}$ gros ist. Leonardo sagt nicht wie er es gefunden hat. Gewiss ist er auch keiner bestimmten vorgeschriebenen Methode gefolgt, sondern er hat - wie es ein geübter Rechner heutzutage auch thun würde - nach und nach die Korrektionen zu den bereits gefundenen Werten geprüft, die bei Berücksichtigung aller vorliegenden Umstände für die zweckmässigsten angesehen werden mussten. Um solche Versuchswerte zu finden, hat er die Regel der zwei falschen Ansätze besessen, die er, wie aus seinen Berechnungen von Kubikwurzeln hervorgeht, als Interpolation $\mathrm{zu}$ benutzen verstand. Als guter Rechner hat er übrigens auch verstanden den Nennern in den durch diese Methode be- 
stimmten Korrektionen solche Abrundungen zu geben, dass seine Methode mehr derjenigen von Vieta geglichen hat, oder wie man sie gewöhnlich nennt, der Newtonschen Methode zur angenäherten Berechnung der Wurzeln einer algebraischen Gleichung. Diese Methode ist im übrigen nur eine Erweiterung derjenigen, die von Leonardo benutzt wurde und die noch benutzt wird für die successive Bestimmung der Ziffern in einer Quadrat- oder Kubikwurzel, und die von den Astronomen seit Ptolemäus benutzt worden war für die Bestimmung der successiven Sexagesimalbrüche einer Quadratwurzel. Es ist vor kurzem nachgewiesen, dass die vorgelegte Gleichung so gewählt ist, dass man, wenn man während der Rechnung Sexagesimalbrüche benutzt und die successiven Korrektionen mit einigem Geschick wählt, verhältnismässig sehr rasch den angegebenen Wert mit seiner kleinen Abweichung vom wahren Werte erreicht.

Nach dieser letzten Bemerkung muss Leonardo die Ehre für die grosse Annäherung vielleicht mit demjenigen teilen, der die Aufgabe gestellt hat. Möglich ist es, dass dies auch Leonardo ist, der in Veranlassung der feierlichen Vorstellung vor dem Kaiser den Magister Johannes inspiriert haben kann; aber dieser, der selbst von Sicilien war und den für arabische Wissenschaft interessierten Kaiser begleitete, kann auch die Aufgabe von arabischen Mathematikern erhalten haben. Da auch Leonardo ein Schüler der Araber war, so erkennt man, dass diese nun die mathematische Wissenschaft so weit gebracht hatten, dass das Ausrechnen eines so feinen Näherungswertes die Kräfte eines hervorragenden Rechners nicht überstieg. Das Beispiel für eine ähnliche Annäherung, das man bei einem arabischen Schriftsteller gefunden hat (S. 310), ist einige Jahrhunderte jünger. 
Dass Leonardo von Pisa in klarer Weise das Wichtigste und Zugänglichste der damals bei den Arabern vorliegenden Mathematik dargestellt hatte, bedeutete indessen nicht zugleich auch, dass die Mathematik nun in den Besitz derjenigen gebracht war, die sich danach in Europa mit dieser Wissenschaft beschäftigten. Die Buchdruckerkunst existierte nicht, und zwischen den Bearbeitern des Faches gab es keinen so lebhaften Verkehr wie ehemals zwischen den weit zerstreuten Griechen. Der damalige gelehrte Stand, die Geistlichkeit, namentlich gewisse Mönchsorden, und dann die aus diesen Kreisen allmählich entstehenden Universitäten dehnten allerdings ihre Verbindungen über die Länder hin aus, aber dieser Stand scheint lange Zeit hindurch nicht von dem beeinflusst worden zu sein, was in italienischen Kaufmannskreisen entstanden war, deren Verbindung mit dem ketzerischen Kaiser Friedrich überdies kaum eine Empfehlung sein konnte.

Wenn wir hier von gelehrten Kreisen und Universitäten gesprochen haben, so darf man dabei nicht an solche Bildungsanstalten denken, in denen z. B. immer in etwas Mathematik unterrichtet wurde. Allerdings gab es bei den Universitäten eine "Facultas artium», an der die erforderliche Vorbildung für andere Studien mitgeteilt wurde, aber diese beschränkte sich in der Regel auf das "Trivium» (woher das Wort «triviell»), das Grammatik, Rhetorik und Dialektik umfasste, und vernachlässigte das "Quadrivium», das Arithmetik, Musik, Geometrie und Astronomie enthielt. Selbst wo man dieses mitnahm, beschränkte sich die Arithmetik auf ein wenig Rechnen. die Geometrie auf eine gewisse äusserst dürftige Durchnahme einiger Bücher von Euklid, über den man noch lange so wenig genau unterrichtet war, dass einige glaubten, seive Elemente wären ursprünglich arabisch geschrieben, 
während andere meinten, dass er nur die Sätze, und der griechische Herausgeber Theon die Beweise geliefert habe.

Indessen gingen aus diesen Kreisen dann und wann Männer hervor, die sich mit Mathematik beschäftigten. Dann aber holten sie sich, wie schon gesagt, ihre Gelehrsamkeit nicht bei Leonardo von Pisa, sondern in einer. Arithmetik und Algebra (De numeris datis) seines Zeitgenossen Jordanus Nemorarius, eines höchst angesehenen Mitgliedes des Dominikanerordens, dessen Ordensgeneral mit dem Hauptsitz in Paris er wurde. Seine Arbeit hatte und verbreitete solche Vorzüge und Mängel, wie wir sie den Algorithmikern beigelegt haben. $\mathrm{Zu}$ diesen fügte er selbst den wesentlichen Vorzug hinzu, überall willkürliche Zahlen durch Buchstaben darzustellen, jedoch so, dass sie in den Wortverbindungen des Textes genannt und nicht zu einer Buchstabenrechnung zusammengestellt werden. Er hat ferner eine geometrische Arbeit über Dreiecke geschrieben, in der er auf der Grundlage von Euklids Elementen verschiedene selbständige geometrische Untersuchungen vornimmt.

Wenn nun auch namentlich die hier erwähnte Arithmetik und Algebra bedeutend hinter Leonardos Liber abaci zurücksteht, so beweist sie andererseits doch, dass man sich auch in den gelehrten Kreisen mit der Aneignung und einer selbständigen Bearbeitung der Mathematik beschäftigte. Diese Beschäftigung wurde an vielen Orten fortgesetzt. Mit ihr verbanden sich fortgesetzte Übersetzungen aus dem Arabischen, später auch aus dem Griechischen. Len ardos Schriften waren wohl auch von einigem Einfluss, teils in Norditalien selbst, wo 300 Jahre später eine neue schöpferische Mathematik sich Bahn brach, teils an anderen Orten, wo sich während des genannten Zeitraumes allmählich auch Fortschritte zeigten. Wir wollen hier jedoch nicht dieser Entwicke- 
lung genauer nachgehen, sondern nur einzelne Beispiele für wirkliche Fortschritte anführen.

Da haben wir denn aus dem 14ten Jahrhundert zwei Arbeiten des Franzosen Nicole Oresme zu nennen. Die eine ist sein Tractatus de latitudinibus formarum. Auf die Ebene übertragen bedeuten hier die Worte Länge und Breite dasselbe wie sonst auf der Kugel, also rechtwinkelige Koordinaten. Diese Benennungen werden besonders dadurch verständlich, dass die Koordinaten innerhalb eines Rechtecks abgetragen werden, das seine grösste Ausdehnung in der Richtung der Abscissen (der Länge) hat. In dieser Figur wird die verschiedene Stärke einer veränderlichen Naturerscheinung, z. B. der Wärme, durch die Ordinaten (Breiten) mit den entsprechenden Zeiten als Abscissen (Längen) dargestellt. Auf diese Weise erhält man eine graphische Darstellung von den Variationen der Wärme in der Zeit durch eine Kurve, und es verdient bemerkt zu werden, dass schon Oresme beobachtet hat, dass die Variation am schwächsten in der Nähe der Maxima und Minima ist. Man sieht, dass die Anwendung von Koordinaten bei Oresme von anderer Natur ist als bei den alten Griechen, auf die er jedoch hinzuweisen scheint, von deren exakter, geometrischalgebraischer Benutzung von Koordinaten zum Studieren der Kegelschnitte und zur Lösung von Aufgaben er aber kaum, weder direkt noch durch die Araber, irgend welche eingehende Kenntnis gehabt haben kann.

Aus dem zweiten Buche von Oresme, das den Titel Algorismus proportionum, führt, wollen wir namentlich die Einführung von Potenzen mit gebrochenen Exponenten erwähnen und die einfachsten Regeln für das Rechnen mit diesen. Oresme gebraucht auch eine besondere Bezeichnung für Potenzen. $4^{1 \frac{1}{2}}$ schreibt er etwa 
so: $\left[1^{p} \frac{1}{2}\right] 4$, wo der Buchstabe $p$ "proportio», Verhältnis, bedeutet. Die Wurzeln dieser Potenzen sind nämlich, da Euklids exakte Proportionslehre zu Grunde gelegt wird, Verhältnisse, und die Potenzen mit ganzen Exponenten werden als zusammengesetzte Verhältnisse gebildet. Übrigens hat die Bildung einer Potenz mit gebrochenem Exponenten auf diesem Wege ein Vorbild bei den Alten, denn Archimedes zeigt, dass das Verhältnis zwischen dem grösseren und kleineren Abschnitt, worin eine Kugel durch eine Ebene geteilt wird, grösser ist als das Verhältnis zwischen den krummen Oberflächen anderthalbmal genommen, d. h. dieses Verhältnis in der Potenz $\frac{3}{2}$. Dadurch dass die Regeln für das Rechnen mit Potenzen von derselben Basis und mit ganzen Exponenten auf Potenzen mit gebrochenen Exponenten erweitert werden, wird Oresmes Arbeit ein Vorläufer für das Rechnen mit Logarithmen.

Das zuerst genannte Werk von Oresme wurde nach Erfindung der Buchdruckerkunst verschiedene Male herausgegeben, und ist gewiss auch vorher ziemlich verbreitet gewesen. Das zweite, sowie dasjenige von Chuquet, das wir jetzt besprechen wollen, sind dagegen erst vor kurzem aus historischem Interesse gedruckt worden, und scheinen keinen sonderlichen Einfluss ausgeübt zu haben. So verrät Chuquet, der auf andere Weise, als es sein Landsmann Oresme 100 Jahre früher gemacht hatte, das Rechnen mit Logarithmen vorbereitet, keine Kenntnis von dessen Arbeit. Beide Arbeiten verdienen jedoch hier erwähnt zu werden, als Zeugnisse für das, was tüchtige Männer unter den vorhandenen Umständen vermochten. Sie zeigen, wie weit man gekommen war.

Die Arbeit von Chuquet, auf die wir hindeuteten, und die uns als Beispiel für eine hervorragende Arith- 
metik und Algebra des 15ten Jahrhunderts dienen soll, führt den Titel «Triparty en la science des nombres». Um zunächst bei demselben Gegenstand zu bleiben, den wir bei Oresme getroffen haben, so treten gebrochene Exponenten indirekt in einzelnen Aufgaben auf, z. B. in der folgenden: Ein Mann reist den ersten Tag 1 Meile, den zweiten 3 , den dritten 9 , u. s. w., wie weit ist er in $5 \frac{1}{2}$ Tagen gereist? Chuquet giebt in der That die Lösung, die man erhält, wenn man voraussetzt, dass die Geschwindigkeit kontinuierlich zunimmt nach demselben Gesetze wie von einem Tage zum anderen. In einer Aufgabe wird geradezu nach einem Exponenten, also nach einem Logarithmus gefragt: Ein Gefäss hat einen Spalt, durch den tägtich $\frac{1}{10}$ vom Inhalt des Gefässes entleert wird; nach Verlauf von wie viel Tagen wird die Hälfte des Inhaltes geleert sein? Die Lösung $6 \frac{31441}{3} \frac{14}{14} 1$ ist diejenige, die durch einfache Interpolation gefunden wird oder durch Anwendung der zwei falschen Ansätze auf die Versuchswerte 6 und 7 . Chuquet selbst ist mit dieser Annäherung nicht zufrieden. An einer Stelle kommt er sogar dazu, formell eine Hauptregel für das Rechnen mit Logarithmen zu geben, indem er eine Reihe von Potenzen der Zahl 2 nebst den zugehörigen Exponenten aufstellt und bemerkt, dass das Produkt von zwei Zahlen der ersten Reihe diejenige Zahl derselben Reihe ist, die der Summe der zu den Faktoren gehörenden Exponenten entspricht.

Wenn Chuquet sein Verständnis von gebrochenen Exponenten nur indirekt zeigt, so treten der Exponent 0 und negative Exponenten in seinen Bezeichnungen bestimmt hervor. Bereits Diophant hatte, wie wir gesehen haben, besondere Bezeichnungen für jede von den Grössen

$$
x^{-6}, x^{-5}, \ldots 1 \ldots x^{5}, x^{6}
$$


4. Erstes Wiedererwachen der Mathematik in Europa. 327

und seine Rechenregeln zeigen, dass er nicht ohne Verständnis für die Übereinstimmung zwischen diesen Grössen war. Chuquet lässt diese Übereinstimmung in der Be. zeichnung selbst hervortreten, indem er die Exponenten der verschiedenen Potenzen der Unbekannten durch ein Exponentenzeichen ausdrückt, das er dem Zahlenkoefficienten, womit diese Potenz multipliciert werden soll, hinzufügt. Dieser Exponent kann positiv, Null oder negativ sein; das letzte wird durch Hinzufügung von $\bar{m}$ bezeichnet. So bedeutet $73^{\bar{m}}$ dasselbe, was wir jetzt $7 x^{-3}$ schreiben. Da Chuquet zugleich eine Bezeichnung für die $n$ te Wurzel hat (jedoch nur für bestimmte Werte von $n$ ), sowie die Zeichen $\bar{p}$ und $\bar{m}$ für unser + und - , so sieht man, dass er imstande war eine recht übersichtliche Darstellung von Gleichungen und dadurch auch von denjenigen von ihren Umformungen zu geben, die man bis dahin in Worten augesdrückt hatte.

Wenn Chuquet, wie wir gesehen haben, sich nicht scheut negative Grössen in seine Exponenten einzuführen, so darf man sich nicht wundern, dass er sich ruhig darin findet, wenn Gleichungen negative Lösungen erhalten, und dass er versteht sie zu erklären. Dass eine von seinen Aufgaben in Wirklichkeit imaginäre Lösungen erhält, scheint dagegen nur auf einem Irrtum zu beruhen.

Indem wir Chuquets gute Behandlung solcher Gegenstände, die wir auch von früheren Mathematikern haben bewältigen sehen, übergehen, wollen wir hier nur noch anführen, dass er, und zwar mit dem Anspruch sie selbst gefunden $\mathrm{zu}$ haben, die bekannte Regel benutzt für die Bildung von einfachen Mittelgrössen $\left(\frac{a_{1}+a_{2}}{b_{1}+b_{2}}\right)$ zwischen zwei bekannten Grössen $\frac{a_{1}}{b_{1}}$ und $\frac{a_{2}}{b_{2}}$. Diese ge- 
braucht er für die Bildung neuer Versuchswerte für die genauere Lösung einer Gleichung, die eine zwischen $\frac{a_{1}}{b_{1}}$ und $\frac{a_{2}}{b_{2}}$ ligende Wurzel hat.

Solche Fortschritte wie die hier erwähnten treffen wir nicht in einem Buche, das von Chuquets Zeitgenossen Luca Paciuolo herrührt, 1494 in Venedig gedruckt wurde und den Titel Summa de Arithmeticu Geometria Proportioni et Proportionalita führt. Dieses Buch bringt im wesentlichen nur das, was früher schon zustande gebracht war, und vieles ist nicht so klar und genau dargestellt wie früher beispielsweise von Leor ardo von Pisa; aber das Gebrachte ist nach grossem Maassstabe angelegt und auf zahlreiche theoretische und praktische Beispiele angewandt. Die Hauptsache aber ist, dass dieses gedruckte Buch verbreitet wurde und in den Händen der verschiedenen Männer war, die in der nächsten Zeit namentlich die Algebra weiterführen sollten. Diese erhielten so einen gemeinsamen Ausgangspunkt und dadurch ein gutes Mittel sich gegenseitig zu verstehen und zusammenzuarbeiten.

Während wir hier auf dem Gebiete der Arithmetik und Algebra im wesentlichen nur Vorbereitungen auf die grossen Fortschritte sehen, die die nächstfolgende Zeit bringen sollte, so treffen wir noch in der hier besprochenen Zeit einen Mann, der auf einem anderen Gebiete selbst mit thätig ist, um solche Fortschritte hervorzubringen, die im Gegensatz zu denjenigen von Chuquet bleibende Bedeutung erhielten. Das ist der Deutsche Johannes Müller, gewöhnlich Regiomontanus genannt. Er ist 1436 geboren und war ein Schüler von Peurbach, Professor in Wien, der sich grosse Verdienste um die Einführung der Trigonometrie des Ptolemäus und der 
Araber in Europa erworben hat. Regiomontanus führte ein sehr bewegtes Leben, hielt sich bald in Italien, bald in Deutschland und Ungarn auf, und bereits in einem Alter von 40 Jahren machte der Tod seiner nach grossem Maassstabe angelegten mathematischen und astronomischen Thätigkeit ein Ende. Sein Umherschweifen wirkte jedoch nicht blos störend, sondern brachte ihn mit vielen Astronomen und Mathematikern in Berührung, und in Italien hatte er Gelegenheit Griechisch zu lernen, namentlich in der Absicht Peurbachs Ausgabe von Ptolemäus' Almagest fortzusetzen. Dabei machte er aus erster Hand Bekanntschaft mit anderen griechischen Mathematikern, die er sehr hoch stellte. Einer von denen, die er kennen gelernt hatte, war Diophant, und er legte sein zahlentheoretisches Interesse, das übrigens gewiss schon vorher durch den Verkehr mit italienischen Mathematikern genährt worden war, dadurch an den Tag, dass er eine Reihe sehr schwieriger hierher gehöriger Aufgaben stellte.

Die grösste Bedeutung haben jedoch seine trigonometrischen Arbeiten erhalten. Unter diesen wollen wir im Anschluss an das, was wir bei arabischen Astronomen getroffen haben und was sein Lehrer Peurbach fortgesetzt hatte, zuerst seine trigonometrischen Tabellen erwähnen. Er ist der erste, der in diesen die Benutzung des Decimalsystemes durchgeführt hat. Seine zuletzt ausgearbeiteten Sinustafeln gehen von Minute zu Minute, und da er den Radius gleich $10^{7}$ setzt, so ist die Genauigkeitsgrenze dieselbe wie in einer 7-ziffrigen Tafel, ohne dass jedoch eigentliche Decimalbrüche schon in Gebrauch gekommen wären.

Die Lehre von der geometrischen Anwendung solcher Tafeln wird bei Regiomontanus zum ersten Male selbständig dargestellt und nicht nur in der Form von Hülfssätzen für die Benutzung in der Astronomie. Den Ge- 
danken, ein solches eigentlich trigonometrisches Werk auszuarbeiten, führt Regiomontanus mit gewohnter Pietät gegen Peurbach auf diesen seinen Lehrer zurück. Sein Buch führt den Titel: De triangulis omnimodis libri quinque. Hierin werden nicht nur, wie bei den früheren Schriftstellern, die rechtwinkeligen Dreiecke behandelt, aus denen die übrigen sich allerdings zusammensetzen lassen, sondern sowohl in der Ebene wie auf der Kugel wird die Beantwortung mancher Fragen, die das schiefwinkelige Dreieck betreffen, durchgeführt. So hat Regiomontanus auf der Kugel gezeigt, wie die Seiten eines Dreiecks die Winkel bestimmen und umgekehrt.

Die drei Jahrhunderte, die auf Leonardo von Pisa folgten, waren hauptsächlich dazu benutzt worden den Kenntnissen und Fertigkeiten, in deren Besitz er sich bereits befand, eine so allgemeine Verbreitung zu geben, dass sie den Ausgangspunkt für eine neue Entwickelung bilden konnten. Diese Grnndlage wurde dadurch verstärkt, dass man nun direkte Kenntnis von den Schriftstellern des Altertums besass, denen man sie hauptsächlich verdankte, nämlich von Euklid und zum Teil von Ptolemäus, und dass man gleichzeitig begonnen hatte eine vorläufige Bekanntschaft mit den Schriftstellern zu machen, die während der folgenden Entwickelung die Führung übernehmen sollten: mit Archimedes, Apollonius und Diophant. Eine wirklich neue Entwickelung war durch Regiomontanus auf dem Gebiete der Trigonometrie begonnen worden. Von den technischen Hülfsmitteln, welche die Algebra benutzen sollte, kannte man allgemeine Zeichen für + und - , nämlich $\bar{p}$ und $\bar{m}$, und einige andere. Chuquets mehr entwickelte Zeichensprache zeigte, wenn sie auch nicht in allgemeinen Gebrauch kam, dass man imstande war sich die Mittel der 
4. Erstes Wiedererwachen der Mathematik in Europa. 331

Darstellung $\mathrm{zu}$ verschaffen, die eine weitergehende Entwickelung verlangen musste.

Hierdurch war eine neue Entwickelungsperiode der Mathematik vorbereitet, die an Fruchtbarkeit nur verglichen werden kann mit den wenigen Jahrhunderten, in denen die griechische Mathematik ihren höchsten Standpunkt erreichte. Diese kam zum Durchbruch, als man durch die Lösung der kubischen Gleichung erkannte, dass man eine Aufgabe bewältigen konnte, die Griechen und Araber hatten liegen lassen müssen, und dadurch ein bisher unbekanntes Vertrauen zu seinen eigenen Kräften erhielt. Mit Hülfe der Buchdruckerkunst konnten die mannichfaltigen Arbeiten, die jetzt in den verschiedenen Ländern entstanden, auf fruchtbringende Weise zusammen wirken. In der Algebra folgten auf den genannten Durchbruch rasch andere grosse Fortschritte. Gleichzeitig lernte man es, die schwierigen griechischen geometrischen Arbeiten zu verstehen; was man hieraus lernte, das setzte man in neue Verbindung mit der Algebra, der man selbst eine neue Form gegeben hatte, und die analytische Geometrie entstand. Ausserdem ging man in der stereometrischen Behandlung der Kegelschnitte viel weiter als die Alten. Man studierte die statischen Arbeiten des Archimedes und schuf selbst die Dynamik. Vom Studium Diophants gelangte man durch ganz neue Untersuchungen zu den merkwürdigsten zahlentheoretischen Sätzen. Das Studium von Archimedes' infinitesimalen Untersuchungen führte dazu, ähnliche Untersuchungen in wachsendem Umfange selbst vorzunehmem und die dazu dienenden Methoden so $\mathrm{zu}$ entwickeln, dass man am Schlusse des 17 ten Jahrhunderts eine Infinitesimalrechnung besass. Die am Schlusse des Mittelalters erwähnten Anläufe führten $\mathrm{zu}$ einer wirklichen Benutzung von Logarithmen. 
Das mag bereits hier am Ende unserer Schilderung von der Geschichte der Mathematik im Altertum und Mittelalter gesagt werden. Man wird dann verstehen, dass der Grund, weshalb wir solange namentlich bei der Mathematik des griechischen Altertums verweilt haben, nicht nur das grosse Interesse ist, das diese an und für sich beansprucht als Glied in der Menge von Kenntnissen, die man nun am Schlusse des Mittelalters gewonnen hatte, sondern zugleich der Umstand, dass sie die Quelle ist, aus der man danach fortfuhr reiche Anregung zu schöpfen zu einer Zeit, wo man gelernt hatte diesen An. regungen nachzugehen und so etwas wirklich Neues hervorzubringen. 


\section{Namen- und Sachregister.}

Bemerkung. Bei den häufiger vorkommende Namen und Begriffen ist immer nur auf die Seiten verwiesen, auf denen genauere Mitteilungen zu finden sind. Auch sind mehrmals Seiten zusammengezogen, wo solche Mitteilungen nur hin und wieder vorkommen.

Abacisten 316.

Abacus 293, 315-317.

Abbassiden 295.

Abûl Wafâ 296, 313 .

Agrimensoren 9.

Ägypten 16, 259, 294.

Ägypter 8-14.

Ahmes 10.

Ähnlichkeit, 4-5, 113, 136, 149. Akademie 18, 20.

Albattâni 313 .

Alchaijâmi 306, 307, 309.

Alchodschandi 312.

Alchwarizmî 298.

Aldschebr 299.

Alexander der Grosse 23, 28, 261.

Alexandria 23-26, 240, 256, 261, $288,294,295$.

Alfachrî 303.
Algebra der Araber 297-312.

- - Inder 260, 278-287.

- geometrische 38-54, 58,

$83, \quad 90,108,109,146-153$,

203-205, 212, 300 .

Algorismus proportionum 324.

Algorithiniker 316, 323.

Algorithmus 297, 298, 315.

Alkarchî 302-307, 312, 317.

Alkâschî 310,312 .

Alkûhî 309.

Almagest 29, 230, 231, 295, 313, 315,329 .

Almamûn 295, 298, 299.

Almansûr 295.

Almukâbala 299.

Alnas awî $301,302$.

Analyse 92-96, 101-105.

Analysis situs 139. 
Annahmen, allgemeine 112.

Ansatz, falscher 11, 247, 275, 277, 317.

- $\quad$ zwei falsche $275,307,317$, $318,320,326$.

Antiphon $69-71,75,227$.

Apagoge 98, 100, 103.

Apollonius 25-28, 58, 81, 106, $166,191,192,198-221,229$, 233, 296, 330.

Araber 293-322.

Archimedes $25-27, \quad 43, \quad 58$, 78--82, 166-199, 216-219, 227-229, 243, 296, 308, 309, 325,330 .

Archimedische Hülfssätze 79, 309. Archimedisches Princip 190.

Archytas 17-21, 84-87, 91, 97. A ris tarch 28, 225-227, 229, 231. Aristäus 27, 198, 213.

Aristoteles 20, 21, 29, 71.

Arithmetik der Araber 297-312.

- - Griechen 32, 36, $58,242-258$.

- $\quad$ Inder 260, 274287.

- geometrische 39-44,

$53,54,242,243$.

Arithmetische Dinge von Diophant 32.

Âryabhatta $260,272,275,288$. Äste der Hyperbel 200, 208, 211. Astronomen, Astronomie 9, 12, 20, $21,27-30,224,225,230-234$, $260,288,313$.

Asymptoten 195, 199, 208, 210, 219.

Athen 17, 18, 21, 22.

Aufgaben 92, 93, 94, 96.

- ebene 83, 212.
Aufgaben, räumliche 83, 86, 212. - unbestimmte; s. Gleichungen, unbest.

Axengleichung 194, 195.

Axiome 112, 115, 116, 119-138.

Babylon, Babylonier 12, 13, 37, 58,62 .

Bachet de Méziriac 255.

Berechnung, numerische 30,57 , $70,222,224,241,261,310$.

Berührungen v. Apollonius, 27.

Berührung zwischen Kreis u. Kugel v. Demokrit 68.

Beweis 23, 93, 101-105; s. auch Exhaustionsb.

Bhâskara Âcârya 260, 275, 278, 287, 288.

Bibliothek, alexandrinische 24, 31, 295.

Binomialkoefficienten 307.

Bolyai 139.

Brahmagupta 260, 271, 286.

Breite 232.

Brennpunkte 210, 211.

Brennspiegel 191.

Brüche 10, 55, 157, 158, 248, 298, 301, 317 .

Bryson 70, 227.

Buchdruckerkunst 325, 328, 331.

Büschel, projectivische 209.

Byzantiner 311.

Cauchy 130.

Chinesen 269, 282, 311.

Chronologie 25.

Chuquet 325-330.

Cissoide 236.

cosinus 12 . 
Cylinder 26, 217, 309.

Cylinderschnitt 195.

Darstellungsform 23.

- synthetische, 92-104.

Data des Euklid 26, 48, 49, 106, $107,153,154,223,224$.

Decimalbrüche $62,329$.

Definitionen bei Archimedes 176177.

- bei Euklid 112,115 $118,123,126,130-$ 133, 140-144, 155.

Demokrit $12,17,68$.

Desargues 216 .

Descartes 88, 238.

Differenzreihen, s. Reihen, arithmetische.

Dinostratus $21,77,78,104$.

Diokles 27, 236, 237.

Diophant, 32, 60, 241--258, 279, 296, 303, 326, 329-331.

Diorismus, 99, 102, 105, 122, 148, 152, 186, 246, 309 .

Dodekaeder 36.

Dreieckszahlen 35, 36, 42, 53 .

Dreiteilung des Winkels $76,79-$ 82, 88-91, 215, 309.

Dschâbir ibn Aflah (Geber) 314.

Durchmesser der Kegelschnitte 201, 202, 206-208.

Durchmesser, konjugirte 199, 208, 211-213.

Dynamik 331 .

Ehene 125, 132-137.

Ecke, 162, 163

Eindeutigkeit 124, 125
Einführung in die Arithmetik v. Nikomachus 32.

Einheit 128, 155, 159.

Einschiebung 27, 75, 79_-82, 88, $184,216$.

Einteilung der Ebene 35.

Ekthese 98, 99.

Eleatische Schule 65.

Elemente 105-108.

Elemente des Euklid 14, 25, 57, 108-114, 315 .

Elemente des Euklid 1stes Buch 90, 91, 108, 113-139.

Elemente des Euklid 2tes Buch $44-54,108,223$.

Elemente des Euklid 3tes Buch 52, 108, 109, 125.

Elemente des Euklid 4tes Buch 108, 109.

Elemente des Euklid 5tes Buch 109, 139-149, 155, 156.

Elemente des Euklid 6tes Buch $47,48,109,149-153$.

Elemente des Euklid 7.-9tes Buch 109, 155-159, 242.

Elemente des Euklid 10tes Buch 41, 109, 158-161, 164, 167, 320. Elemente des Euklid 11tes Buch 109, 126, 130, 161-163.

Elemente des Euklid 12tes Buch 109, 163, 166-173, 180, 232. Elemente des Euklid 13tes Buch 109, 163-165.

Elemente des Euklid, 14tes und 15tes Buch 108, 113, 165-166.

Elimination 249.

Ellipse 183, 191-207.

Ellipsoide 183, 219.

Eratosthenes 24, 27, 28, 87, $227,232,243$. 
Eudemus 22, 32-34, 39, 72, 73. Geometrie, nicht euklidische 135,

Eudoxus 20, 21, 28, 37, 69, 70, $78,86-89,108-110,140,141$, $166,177,224,225,236$.

Euklid 25-26 und zerstreut im ganzen Buche.

Eutokius 186, :309.

Evolute 221.

Exhaustionsbeweis 69, 78, 110, 111, 141, 166-188, 228.

Exponenten $325-327$.

\section{Facultas artium 322.}

Fehlschlüsse von Euklid 26, 111. Fermat 174, 258.

Fingerrechnung 265.

Flächenanlegung $35,37,39,45$, $46,51,52,150$, 154, 204.

- $47,98,104$.
- $\quad 45$,
$\quad 45,49,52$.
krumme, 176,
184, 237.

Flächensatz 206, 208.

Flächenschnitt 27, 210.

Friedrich II, Kaiser 318, 322.

Fünfeck, regelmässiges $6,36,52$, 108, 109, 164.

Fünfzehneck, regelm. 108.

Geber (Dschabir ibn Aflah) 314. Geodäsie 25, 29.

Geographie 25, 28.

Geometrie, analytische, 94, 106, $202,205,214,238,331$.

- berechnende, 221$232,241$.
139.

- projektivische 26, 134, 135.

- des Raumes 86, 237.

- sphärische 232-235.

Gerade 118-126, 131-136.

Gerbert 316 .

Gesellschaftsrechnung 275

Gleichgewicht, Lehre davon bei Archimedes 26, 177, 187-191, 331.

Gleichung, die doppelte des Diophant 2̌1, 319

Gleichungen 1. Grades, 10, 44, $153,275,300,317$.

1. Grades, unbestimmte, 255, 281 $-283.317$.

2. Grades, 35-37, 47-53, 97, 107, 148-154, 205, 215, $280,281,296,300$. $301,304,318$.

2. Grades, numerische, 54-64, 222, 242, 257.

2. Grades, reine 52 .

2. Grades, unbestimmte $41,5 \dot{4}$, $59,60,242,243$, $249-258,283-$ $286, \quad 303, \quad 317-$ 319

3. Grades, 82, 185, 212, 216-219, $307-310, \quad 330$, 331.

4. Grades, 216. diophantische 255 
Gnomon $41-49,98,99,113,245$. Jahrhundert, 5tes, 15-17, 34, 45, Grade 12, 28.

Gradmessung 25, 227, 298.

Grössen, inkommensurable und irrationale $34-39,44,53-$ $62,69,70,110,111,141$, 158-161, 250-256, 279, $305-307,320$.

- kommensurable 37, 64, $155-159$.

- negative $38,280,327$.

Grössenbegriff 127, 128.

Guldin 237.

Halbierung 298.

Harpedonapten 12, 259.

Hârûn Arraschid 295.

Hau-Rechnung 10.

Hebel 187, 188.

Hermotimus 106.

Hero $30,61,62,222,223,287$, 293, 296.

Hiero 26.

Hipparch 28, 29, 230, 230, 233, $234,288,296,300$.

Hippias 17, 76 .

Hippokrates von Chios 17, 22, 71-74, 80, 84, 105.

Hippopede 236.

Höhenmessung durch Schatten 33. Hülfsmittel, analytische 105-108. Hülfssätze, des Pappus 211, 238. Hydrostatik 190.

Hyperbel 87, 191-207, 218-221. konjugierte 208, 211.

Hyperbeläste 209, 211, 213. Hyperboloide 183, 219. Hypsikles 27, 108, 165.

Jahrhundert, 6tes v. Chr. 15, 32, 34. 162-165, 200, 217.

$67,70,72,79,82$, 88.

Ikosaeder 36.

Infinitesimalrechnung 331.

Infinitesimaluntersuchung 63-70, 141, 166-187, 331.

Inhalt einer krummen Fläche 176, $184,237$.

Integral, bestimmtes 173,175 .

Integrationen 181, 183, 189, 221.

Interpolation 231, 275, 307, 313, $320,326$.

Involution 215, 216.

Johannes von Palermo 318, 321.

Jordanus Nemorarius 323.

Irrrationalität, s. Grössen, irrat.

$$
\begin{gathered}
\text { doppelte 57, } 161 \text {, } \\
165,279 .
\end{gathered}
$$

Kegel 68.

Kegelschnitte 18, 26, 27, 53, 81, $82,87,178,186$, 191-121, 237, 308, $309,331$.

- ähnliche 211.

Kettenbrüche 60, 281, 282.

Kleinasien 15, 22.

Kolumnen 266, 267, 293, 316.

Kombinationen 287.

Konchoide 82, 236.

Kongruenz 113, 128-131, 136.

Konklusion 101, 103.

Konoide 26, 181, 183, 184, 195.

Konstantinopel 292.

Konstruktionen 5, 6, 12, 70-80, $88-91,96-103,120,121$, 
Konstruktionsmittel 81.

Konturen, geschlossene 133-139.

Konvergenz 173-176.

Koordinaten, rechtwinkelige 5, 324. sphärische 232.

Koppernikus 28.

Körper, platonische 20.

- die auf e. Flüssigkeit schwimmen, 26.

Kreis 118-125, 134.

Kreisfunktionen 78 .

Kreismessung 26, 227.

Kreisschnitte am Kegel 196, 233. Kubikwurzeln 83, 86, 275, 305$308,316,317$.

Kubikzahlen 13, 43, 62, 244, 287.

Kugel und Cylinder 26, 216, 219, 309.

Kugeloberfläche 184, 186, 237.

Kugelteilung 185, 216, 218, 308. Kurven, spirische 236.

Kyzikos 20, 21.

Lagrange 114, 286.

Landmesser, ägyptische 222. römische $9,11,53$, 293.

Landmessung 29, 30, 222.

Länge einer krummen Linie 176. Länge von Orten 227, 232.

Latitudinibus, de, 324.

Legendre 136, 137.

Leibniz 174.

Lehrgebäude, synthetisches 108, 114.

Lemniskate 236.

Le on 105.

Leonardo (Fibonacci) von Pisa $315-323,328$.
Liber abaci des Leonardo 316$318,322$.

Lîlâvatî $260,275-279$.

Lineal 75, 80, 81, 97, 120, 198.

Lobatschewsky 139.

Logarithmen 325, 326, 331.

Logistik, 29, 241.

Maass, grösstes gemeinschaftliches 55, 56, 156, 159, 282. Maxima 100, 186, 211, 217, 324. Mechanik 6, 29, 190.

Mehrdeutigkeit 100, 101.

Menächmus 21, 87-90, 191193, 197-199.

Menelaus 29, 230, 234, 313.

Methode, analytische 92-104, 106.

- $\quad$ apagogische 92.

- cyklische 285.

Minima 100, 211, 217, 324.

Minuszeichen 248, 327, 330.

Mischungsrechnung 191-275.

Mittel, arithmetisches 148.

- geometrisches (siehe mittl.) Proportionale).

Möglichkeitsbedingungen 91, 96.

Möndchen des Hippokrates 72-74. Muhammed ibn Mûsâ Alchwarizmi 298, 301, 316.

Müller, Joh. (Regiomontanus) 328.

Multiplikation, Fouriersche 274.

Musik 36.

Museum 24.

Näherungsformeln 30, 288.

Näkerungswerte $54,56,229,276$, 281, 282, 288, 307, 310, 320, 321. 
Neunerprobe 298.

Newton $174,321$.

Nikomachus $32,43,236,244$, 293.

Nikomedes 27, 82, 88.

Normale 200, 216, 220, 221.

Null 262, 266, 271, 272, 316, 317.

Oberflächenörter 26.

- Omar 295.

'Omar Alchaijâmi 306.

Optik 26.

Oresme, Nicole $324,325,326$.

Ort zu drei Geraden 213, 214.

- vier - 213-215, 237.

Örter, ebene 106, 212.

- geometrische 86, 94, 97, 106, 117, 198, 206, 212.

- räumliche $27,128,200,212$. Oströmer 292.

Paciuolo, Luca 328.

Pappus 31, 153, 166, 211, 216, 237-239.

Parabel 87, 191, 195, 197, 204, 207, 218-220.

Parabelsegment 171, 173, 189

Paraboloide 183, 190.

Parameter 193, 194.

Pentagramm 35.

Periode, astronomische, 282.

Peripheriewinkel aufd. Halbkreis32.

Permutationen 276 .

Perseus 27, 236.

Perspektive 26.

Peurbach 328, 329, 330.

Phaenomena 26.

Philosophie, Philosophen 17-25, $65,88$. $\pi$ 12, 76, 226-229, 288, 301.

Planetarium 191.

Plato $16-21,35,41,57,68,87$, $88,92,97,224$.

Platoniker $88,89$.

Pluszeichen 327, 330.

Polare 208.

Polyeder, halbreguläre, 26, 166.

- reguläre, $19,27,34,36$, $110-113,161-166$.

Polygonalzahlen 32, 43, 242, 244. Polygone, reguläre, 69, 108, 109, 164, 224-227.

Porismen 26, 215, 234.

Positionssystem 262, 266, $270=$ 273, 298, 301, 317.

Postulate 112, 115-126, 132-139, 162, 176. 177, 192.

Potenz eines Punktes 52, 109.

Potenzen 146, 158, 248, 264, 305, 306, 324, 327.

Potenzsätze 197, 199, 208, 214.

Practica geometrica des Leonardo 218.

Primzahlen 156, 158, 243. relative 156,157 .

Problem, delisches 83, 84, 191, 197.

Probleme 88-91, 99, 102, 103, 104, 106, 111, 112, 120, 122.

Produkt, 40, 44, 145, 146.

Projektion. stereographische 2.33

Proportionalen, zwei mittlere, 84 , $87,88,91,97,146,191,192$, 195.

Proportionen 37, 50 52, 77, 84, $108-110,128,139-$ 156, 302, 306, 325

- $\quad$ arithmetische 35 .

- $\quad$ fortlaufende $146,158$. 
Proportionen, geometrische, 35 . harmonische, 35 .

Protase, 98, 99, 101.

Ptolemäer 24, 31.

P tol e mäus 29, 62, 229-235, 261, 287, 288, 295, 312-315, 321, 328-330.

Ptolemäus Lagi 23.

Pyramidalzahl 44, 243, 244.

Pyramide 163, 169-171, 173.

Pythagoras $16,34,36,39,41,64$.

Pythagoreer, 17, 18, 32-39, 42, $51,64,65,242,244,311$.

Pythagoreischer Lehrsatz, 35, 37, 47-53, 59, 109, 113, 153.

Quadratur des Kreises 69-78, 91, 228.

- der Möndchen 72, 74 . - der Parabel 26-178.

Quadratrix 76-79, 91, 226, 237. Quadrattafeln 13, 62, 274.

Qvadratwurzel 51, 54-64, 110 , 227-230, 275, 280, 305-307, $314,316,317$.

Quadratzahlen 13, 40-43, 71, 182, 249-255, 286, 287, 312, 318, 319.

Quadrivium 322.

Quotientenreihe, s. Reihen, geometrische.

Raumkurve 85, 86.

Rechenbrett 58, 265, 293.

Rechenbuch 10, 315 .

Rechenkunst, arabische, 298.

- griechische 29, 57, $58,294$.

- indische, 259-261,
272-274, 290-303, $316,317$.

Rechenmaschinen 265.

Rechenpfennige 265-267.

Rechentafeln 273.

Regel des falschen Ansatzes 11, 247, 275, 277, 317.

Regel der zwei falschen Ansätze 275, 307, 317, 318, 320, 326.

Regel der Umkehrung 276, 277, 317.

Regiomontanus (J. Müller) 328 , $329,330$.

Regula de tri $222,275,277,278$, 302.

Reihen, arithmetische 10,36, 42$44,182,276,317$.

- geometrische, 10,67, 146, $147,158,317$.

- unendliche 171-175, 181.

Reihen von Quadratzahlen 182, 287, 312.

Reihen von Kubikzahlen, 244, 287, 312.

Reihen von Biquadraten 312

Resolution 99, 100, 101, 102, 107.

Römer 31, 244, 265-269, 292, $295,315,316$.

Sammlungen, mathem. d. Pappus 31.

Sandrechnung d. Archimedes 26, $58,264,270$.

Sanskrit 259.

Satz des Ptolemäus 230, 231.

Schnitt, bestimmter 27, 215.

Schnitte am Kegel 196.

Schnittpunkte zw. zwei Kegelschnitten 211. zw. Kreis und Ge- 
rade etc. 121,125 , 135.

Schraubenfläche 237.

Schule, akademische 20.

- alexandrinische 15, 21.

- atomistische 67 .

- eleatische 65 .

- jonische 15 .

- peripatetische 20, 22.

Schwerpunkt 178, 188, 189, 237.

Sehnentafeln 62, 229--231, 287, 288, 312.

Sexagesimalbrüche $230,310,316$, $320,321$.

Sexagesimalsystem 12, 13, 28, 58, 62, 264 .

Simplicius 73.

Sinustafeln 231, 287, 288, 312, 313, 329.

Sokrates 18, 19.

Sophismen 65, 66.

Sophisten 17, 23, 69, 70, 71.

Sphäroide 26, 181, 183, 184, 195.

Spirale, archimedische 26, 43, 78 $-80,181-184$.

- sphärische 237.

Stammbrüche 10.

Stereometrie, elementare 110, 126, 130, 161-166.

Strecken, über inkommensurable etc. v. Demokrit 68.

Subnormale 220.

Substitutionen 251.

Süditalien 15-22.

Summation, siehe Reihen.

Sûrya Siddhânta 260, 288.

Symmetrie 130.

Synthese 92-95, 100, 101, 105, 114.

Syrakus 26, 191.
Tangenten 184, 199, 202, 207, 209 -211, 219.

Tangententafel 313 .

Tannery, Paul 8, 73.

Teilung der Figuren 26.

- stetige 56, 109, 152, 164.

Thâbit ibn Kurra 311.

Thales 15, 32, 33 .

Theätet 19, 57, 110, 158.

Theon 323.

Theoreme 88-91, 99, 102-106, 111, 112.

Transformation 98, 101-103, 107. triangulis omnimodis, de 330.

Trigonometrie 29, 223-232, 287, $311-315,328-330$. sphärische 232-235, 314.

Triparly en la science etc. 326. Trivium 322.

Umkehrung 276, 277, 317.

Unendliche, das; s. Infinitesimaluntersuchung.

Unendlichkeit d. Zahlenreihe 58.

Universitäten 322 .

Verdoppelung 298.

des Würfels $82-88$, 91.

Verfall d. griech. Geometrie 235 -241 .

Verhältnis s. Proportionen.

- zusammengesetztes 144-147, 238.

Verhältnisschnitt 27, 210.

Verlegungsaxiom 131-137.

Vieta 82 .

Vîjaganîta 260, 278. 
Volumen 110, 183-186, 237.

Vorgeschichte 1.

Vorzeichen 218, 280.

Voraussetzungen, geometrische, des Euklid 115-131.

- der Geometrie 132 -139 .

Wasserschnecke 191.

Wertheim 247.

Westaraber 314, 315.

Winkel 36, 119, 223-227.

Winkelsumme des Dreiecks 35, 113, 136-138.

Wulst 85, 236.

Zahl als Princip der Dinge 36, 64 . Zahlbenennung 263-274.

Zahlbezeichnung 10, 57, 58, 262 -274 .

Zahlen, ähnliche 40, 42, 43.

- befreundete 36,311 .

- cyklische 71 .

- ebene 40 .
Zahlen, ganze $37-40,60,64,65$, 155, 255, 284-286.

- gerade 42 .

- räumliche 43 .

- ungerade $41,42$.

- vollkommene $36,158,242$, 311.

Zahlen, über die, v. Demokrit 68. Zahlenrechnen 7, 30, 263-278, 298.

Zahlenspekulationen 13.

Zahlentheorie 37, 158, 242, 243, $255,258,278-287,311,312$, 331

Zauberquadrate 311 .

Zehnersystem 263, 264.

Zeichensprache, algebraische 247, $248,257,281,203,324-327$, 330 .

Z eno 65, 66, 67, 71.

Zinsrechnung 275, 317.

Zirkel und Lineal 75, 80, 81, 96, 120, 198.

Zwanzigersystem 263, 264. 


\section{Inhaltsübersicht}

Einleitung.

1. Vorgeschichte der Mathematik ................. 1

2. Ägypter und Babylonier.................... 8

Die griechische Mathematik.

1. Historischer Überblick .................... 14

2. Die pythagoreische Mathematik ................ 32

3. Die geometrische Arithmetik................. 40

4. Die geometrische Algebra .................. 44

5. Numerische quadratische Gleichungen; Ausziehen der Quadratwurzel ........................... 54

6. Das Unendliche ........................... 64

7. Die Quadratur des Kreises $. \ldots \ldots \ldots \ldots \ldots \ldots \ldots \ldots . \ldots \ldots$

8. Dreiteilung des Winkels; Einschiebungen ........... 79

9. Verdoppelung des Würfels ................. 82

10. Probleme und Theoreme; Bedeutung der geometrischen Konstruktion .......................... 88

11. Die analytische Methode; die analytisch-synthetische Darstellungsform........................... 92

12. «Elemente»; analytische Hülfsmittel ............ 105

13. Überblick über Euklids Elemente; synthetisches Lehr-

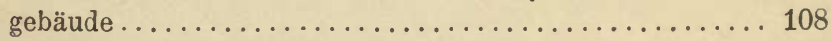

14. Euklids geometrische Voraussetzungen ........... 115

15. Anmerkung über die Voraussetzungen der Geometrie... 132

16. Die allgemeine Lehre von den Proportionen; Euklids 5tes und 6tes Buch......................... 139

17. Kommensurable Grössen und ihre Behandlung durch Zahlen; Euklids 7.-9. Buch ............... 155 
18. Inkommensurable Grössen; Euklids 10tes Buch ....... 158

19. Elemente der Stereometrie; reguläre Polyeder; Euklids 11tes und 13tes Buch ....................... 161

20. Der Exhaustionsbeweis; Euklids 12tes Buch.......... 166

21. Infinitesimale Bestimmungen bei Archimedes ........ 177

22. Archimedes' Lehre vom Gleichgewicht............. 187

23. Die Lehre von den Kegelschnitten vor Apollonius ...... 191

24. Die Kegelschnitte des Apollonius................. 199

25. Räumliche Örter und Aufgaben ................ 212

26. Die berechnende Geometrie ................. 221

27. Sphärische Geometrie.................... 232

28. Verfall der griechischen Geometrie .............. 235

29. Die spätere griechische Arithmetik; Diophant........ 242

Die indische Mathematik.

1. Kurzer Überblick ......................... 259

2. Zahlbenennung, Zahlbezeichnung und Zahlenrechnen vor

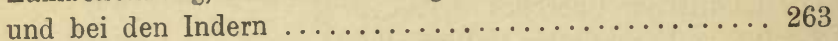

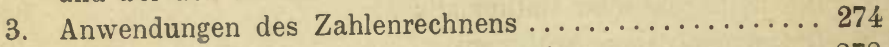

4. Algebra und Zahlentheorie; Geometrie............ 278

Das Mittelalter.

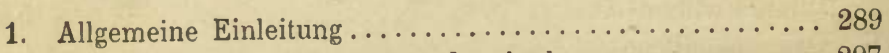

2. Die Arithmetik und Algebra der Araber .......... 297

3. Die Trigonometrie der Araber ................ 312

4. Erstes Wiedererwachen der Mathematik in Europa..... 315 


\section{.}

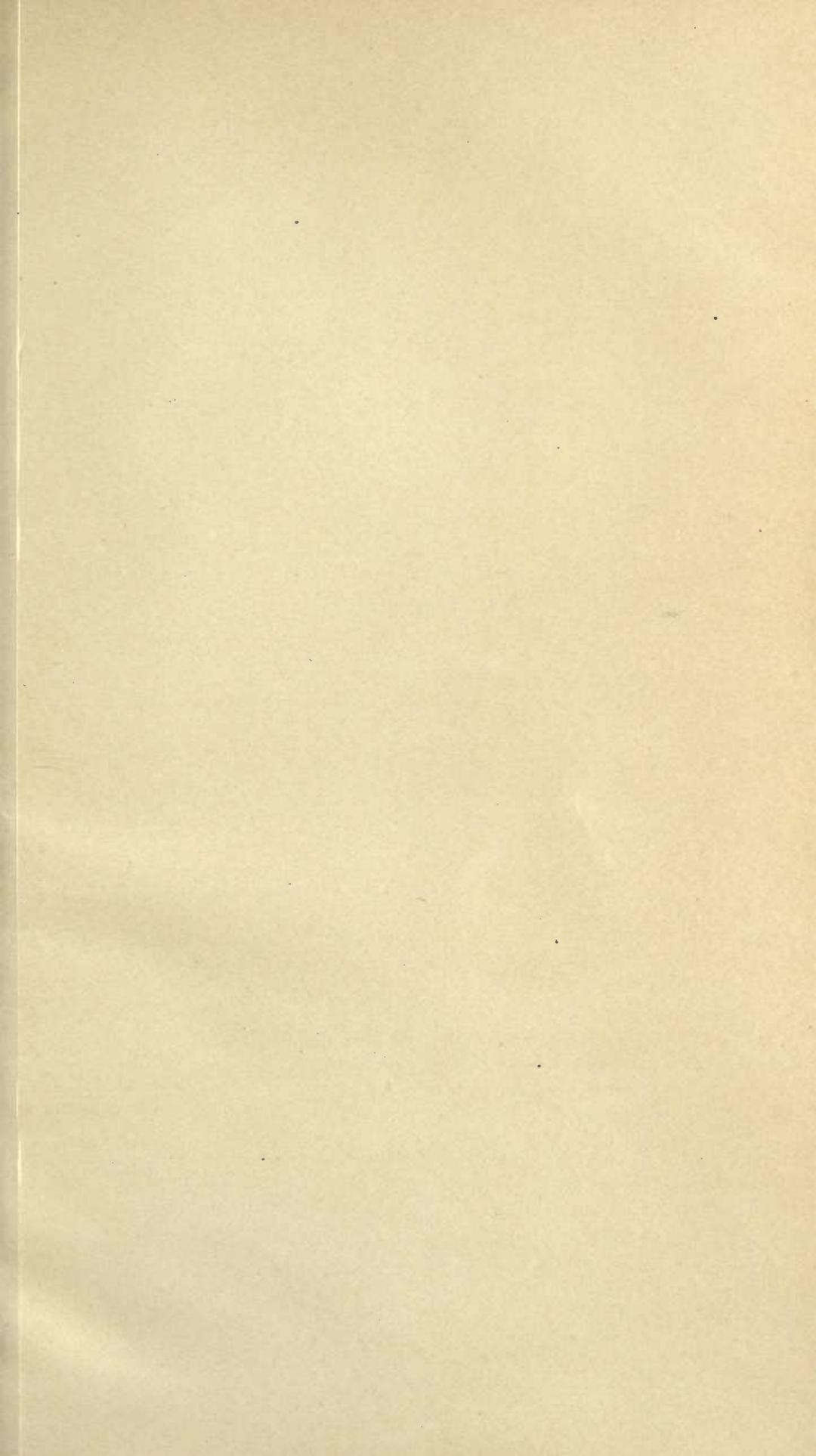





\section{SEVEN DAY RESERVE BOOK}

Return to desk from which borrowed.

This book is due on the LAST DATE

stamped below. MATH.-STAT.

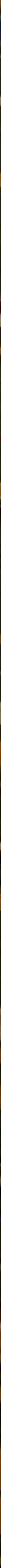



